\title{
THE DEVELOPMENT OF A PASSIVE, WIRELESS SENSOR SYSTEM
}

\author{
by
}

\author{
Natsuki Hasegawa
}

A thesis submitted to Victoria University of Wellington in fulfilment of the requirements for the degree of

Master of Science in Electronic and Computer Systems Engineering.

Victoria University of Wellington

2008 
VICTORIA UNIVFOSIT:

WELLINGTON 


\section{Contents}

1. Introduction 1

2. Background 5

2.1 Introduction 5

2.2 Electronic Article Surveillance 5

2.3 Magnetoelastic Tags 8

2.3.1 Antitheft Applications 8

2.3.2 Magnetoelastic Materials 10

2.3.2.1 Ferromagnetism 13

2.3.2.2 Domain Theory 13

2.3.2.3 Hysteresis $\quad 15$

2.3.2.4 Magnetic Anisotropy 16

2.3.2.5 Magnetostriction $\quad 19$

2.3.2.6 $\triangle \mathrm{E}$ Effect $\quad 20$

2.3.3 Modelling the Material 21

2.3.3.1 Mass Loading of Magnetoelastic Sensors 22

2.3.3.2 Temperature Dependence $\quad 24$

2.3.4 Detection Methods 26

2.3.4.1 Magnetic Method $\quad 26$

2.3.4.2 Acoustic Method 27

2.3.4.3 Optical Method 27

2.3.4.4 Impedance De-tuning Method 27

2.3.5 Literature Survey of Magnetoelastic Sensor Applications 28

3. Design and Construction of a Handheld Magnetoelastic Sensor Reader

3.1 Introduction 31

3.2 System Overview 31

3.3 Microcontroller 34

$3.4 \quad$ LCD and Keypad $\quad 38$

$\begin{array}{lll}3.4 .1 & \text { LCD } & 38\end{array}$ 
$\begin{array}{ll}3.4 .2 \text { Keypad } & 39\end{array}$

3.5 Waveform Generator 41

3.5.1 Circuit Setup 43

3.5.2 Programming the Waveform Generator 45

3.5.2.1 Control Register 46

3.5.2.2 $\mathrm{F}_{\text {start }}$ and $\Delta F$ Registers $\quad 47$

3.5.2.3 Number of Increments $\quad 48$

3.5.2.4 Burst Interval $\quad 48$

3.6 Power Amplifier $\quad 49$

3.6.1 Circuit Setup 50

3.7 Operational Amplifier Circuits 51

3.7.1 Circuit Setup 53

3.8 Voltage Comparator 53

3.8.1 Circuit Setup 54

3.9 Power Supply and Grounding 55

3.9.1 Circuit Setup 56

3.10 Reader and Drive Coils $\quad 56$

3.11 Printed Circuit Board Design 58

3.11.1 Debugging the Board 60

3.12 Completed System 63

3.13 Performance of System 64

3.13.1 Detection Distance 65

3.13.2 Offsetting from Centre 67

3.14 Possible Improvements $\quad 70$

$\begin{array}{ll}\text { 4. Development of Magnetoelastic Sensors } & 71\end{array}$

$\begin{array}{lll}4.1 & \text { Introduction } & 71\end{array}$

4.2 Experimental Procedure for Impedance De-tuning 72

4.2.1 Sensor Response as a Function of Magnetic Biasing Field 74

$\begin{array}{lll}4.3 & \text { Biasing Magnet } & 75\end{array}$

4.3.1 Magnetising the Strip 76

4.3.2 Distance from Strip 78

4.3.3 Sensor Response with Biasing Magnet $\quad 79$

$\begin{array}{lll}4.4 & \text { Temperature Dependence } & 81\end{array}$ 
4.5 Preparation of Humidity Sensor 84

4.5.1 Centrifuge 84

4.5.2 Polypyrrole (PPy) 85

4.5.3 Polyvinyl Alcohol (PVA) 86

4.6 Experimental Arrangement and Procedure for Humidity Tests 87

4.7 Initial Evaluation of Humidity Sensor Response 91

4.7.1 Response of Uncoated Sensor Strip 91

4.7.2 Polypyrrole (PPy) 92

4.7.3 Polyvinyl Alcohol (PVA) 94

4.7.4 Discussion 95

4.8 Construction of a Prototype System for Humidity Measurement 95

4.8.1 Design of Sensor Element 96

4.8.2 Evaluation of Humidity Sensor Response 97

4.8.3 Using the Handheld Reader for Humidity Measurement 99

$\begin{array}{ll}4.8 .4 \text { Calibration } & 101\end{array}$

4.8.5 Applied Humidity Measurements 102

4.8.5.1 Plastic Food Container $\quad 102$

4.8.5.2 Wall Cavity 103

$\begin{array}{lll}4.9 & \text { Sensor Stability } & 107\end{array}$

4.10 Construction of a Magnetoelastic Sensor Array 110

4.10.1 Milling 111

4.10.2 Etching 112

4.10.3 Characterisation of Constructed Sensor Array 115

$\begin{array}{ll}\text { 4.10.4 Discussion } & 117\end{array}$

$\begin{array}{ll}\text { 5. Summary and Conclusions } & 119\end{array}$

$\begin{array}{lr}\text { Appendices } & 123\end{array}$

Appendix A: Schematic Diagrams 123

$\begin{array}{ll}\text { Appendix B: PCB Layout } & 124\end{array}$

Appendix C: Microcontroller source code $\quad 125$

$\begin{array}{lr}\text { References } & 147\end{array}$ 


\section{List of Figures and Tables}

Figure 1.1: Damage resulting due to water penetration in building structures. 2

$\begin{array}{ll}\text { Figure 2.1: Reusable antitheft tags attached to store items. } & 6\end{array}$

Figure 2.2: EAS detectors found at a bookstore.

Figure 2.3: Excitation and sensor response signals.

Figure 2.4: Commercial magnetoelastic EAS tag. 9

$\begin{array}{ll}\text { Figure 2.5: Production of amorphous metal ribbons. } & 10\end{array}$

Figure 2.6: Smooth and rough sides of Metglas 2826MB ribbons. 11

Figure 2.7: Examples of products made from amorphous metal alloys. $\quad 12$

Figure 2.8: Reduction of magnetostatic energy through domain formation. $\quad 14$

Figure 2.9: Magnetic domains in an amorphous metal ribbon. 14

Figure 2.10: Hysteresis loop showing common magnetic properties. 15

Figure 2.11: Hysteresis loop of Metglas 2826MB. 16

Figure 2.12: Magnetocrystalline anisotropy of magnetite. 17

Figure 2.13: Schematic showing the external field and demagnetising field. 17

$\begin{array}{ll}\text { Figure 2.14: Sensor aspect ratio versus optimal biasing field. } & 18\end{array}$

Figure 2.15: Schematic of a material exhibiting positive magnetostriction. $\quad 19$

Figure 2.16: Stress-strain curves of ferromagnetic and non-magnetic materials. 21

Figure 2.17: Inverse of sensor length versus resonant frequency. 22

Figure 2.18: Previous results with PPy and PVA coated sensors. 24

Figure 2.19: Previous results with temperature dependence. 25

Figure 2.20: Methods to detect magnetoelastic sensors. 26

Figure 2.21: Impedance and phase plot of a reader coil with a Metglas 2826MB strip inside. 28

Figure 2.22: Detection system designed by Ong et al. 29

Figure 2.23: Measurement of $\mathrm{pH}$ with magnetoelastic sensors. 30

Figure 2.24: Detection of bacterial spores with magnetoelastic sensors. $\quad 30$

Figure 3.1: Block diagram of sensor reader. 32

Figure 3.2: Driving signal, sensor response and total response signals. 33

Figure 3.3: Driving frequency versus ring-down time. 34

Figure 3.4: Program flow of microcontroller. $\quad 40$

Figure 3.5: Connection for the pushbuttons. 41 
Figure 3.6: Schematic of waveform generator circuit.

Figure 3.7: Schematic of power amplifier circuit. 50

Figure 3.8: Amplified but unfiltered signal. $\quad 52$

Figure 3.9: Schematic of op amp circuit. 53

Figure 3.10: Illustration showing voltage comparator operation. 54

Figure 3.11: Schematic of voltage comparator circuit 54

Figure 3.12: Schematic of power supply circuit. 56

Figure 3.13: Difference in signal quality after filtering and amplification for not $\begin{array}{ll}\text { spaced and spaced coils. } & 57\end{array}$

Figure 3.14: Reader probe consisting of the drive coil and reader coil. 57

Figure 3.15: Completed PCB of reader mounted in its case. 59

Figure 3.16: Terminal block connector for the reader and drive coils. $\quad 59$

Figure 3.17: Constructed op amp circuit with a prototyping board. 62

Figure 3.18: Completed reader system with case open. 62

Figure 3.19: Completed reader system with case closed. 64

Figure 3.20: Setting the reader into programming mode. 64

Figure 3.21: Measuring the effect of distance from reader coil. 65

Figure 3.22: Distance versus mean number of detected sensor pulses. 66

Figure 3.23: Distance versus mean of measured resonant frequencies. 67

Figure 3.24: Measuring the effect of offsetting from the centre of the reader coil. 68

Figure 3.25: Offset distance versus mean number of detected sensor pulses. $\quad 69$

Figure 3.26: Offset distance versus mean of measured resonant frequencies. 69

Figure 4.1: Experimental arrangement for impedance de-tuning. 72

Figure 4.2: Current versus magnetic field strength of Helmholtz coil. 73

Figure 4.3: Position versus magnetic field strength of Helmholtz coil. $\quad 74$

Figure 4.4: Biasing magnetic field strength versus resonant and anti-resonant frequencies as well as relative signal strength. $\quad 75$

Figure 4.5: Side of the sensor showing Metglas strip, spacer and biasing magnet. 76

Figure 4.6: DC coil used to magnetise the spring steel strip.

Figure 4.7: Coil current versus field strength for the magnetising coil. 78

Figure 4.8: Distance from strip surface versus magnetic field strength. $\quad 79$

Figure 4.9: Biasing magnetic field strength versus resonant frequency with Helmholtz coil and $40 \mathrm{~mm}$ bias magnet. 
Figure 4.10: Biasing magnetic field strength versus relative signal strength with

$\begin{array}{ll}\text { Helmholtz coil and } 40 \mathrm{~mm} \text { bias magnet. } & 81\end{array}$

Figure 4.11: Heated food container used to measure temperature dependence. 81

Figure 4.12: Temperature versus resonant frequency for three biasing fields. 82

Figure 4.13: Temperature versus resonant frequency with biasing magnet. 83

Figure 4.14: Centrifuge used as a spin coater. 85

Figure 4.15: Chemical structure of PPy and Metglas strip coated with PPy. $\quad 86$

Figure 4.16: Chemical structure of PVA and Metglas strip coated with PVA. $\quad 87$

Figure 4.17: Gas flow system constructed by Holt and Zhen. 87

Figure 4.18: Glass test cell. $\quad 88$

Figure 4.19: LabView user interface to read the relative humidity and the Honeywell HIH-4000 IC humidity sensor. $\quad 90$

Figure 4.20: Calculated relative humidity versus values measured by the IC sensor. $\quad 90$

Figure 4.21: Experimental arrangement for humidity testing. 91

Figure 4.22: Relative humidity versus resonant frequency for uncoated sensor. 92

Figure 4.23: Relative humidity versus resonant frequency for PPy coated sensor. 93

Figure 4.24: Relative humidity versus resonant frequency for PVA coated sensor. 95

Figure 4.25: Constructed humidity sensor. 97

Figure 4.26: Relative humidity versus resonant frequency for the PVA coated sensor using the Helmholtz coil and biasing magnet. 98

Figure 4.27: Relative humidity versus relative signal strength for PVA coated sensor using the Helmholtz coil and biasing magnet. 98

Figure 4.28: Positioning of the reader coil for the sensor reader system. $\quad 100$

Figure 4.29: Relative humidity versus resonant frequency for PVA coated sensor using impedance de-tuning as well as the reader. $\quad 100$

$\begin{array}{ll}\text { Figure 4.30: Calibrating the humidity response. } & 101\end{array}$

Figure 4.31: Measurement of humidity inside the sealed food container. $\quad 102$

Figure 4.32: Humidity measured by IC sensor versus magnetoelastic sensor inside sealed food container. 103

Figure 4.33: Constructed wall model for practical humidity measurement. $\quad 104$

Figure 4.34: Humidity sensor placed inside the wall cavity. 104

Figure 4.35: Measurement of humidity inside the wall cavity. 105

Figure 4.36: Humidity measured by IC sensor versus magnetoelastic sensor inside wall cavity. 
Figure 4.37: Corrosion of the sensor strip.

Figure 4.38: Relative humidity versus resonant frequency for a new sensor and an aged sensor.

Figure 4.39: Relative humidity versus relative signal strength for a new sensor and an aged sensor.

Figure 4.40: Four element sensor array design in Altium Designer.

Figure 4.41: Milling machine and failed attempt at cutting the Metglas material. 112

Figure 4.42: PCB etching machine used to shape Metglas 2826MB. 113

Figure 4.43: Yellow mounting wax used to secure the strip to the copper board. 113

Figure 4.44: Completed Metglas sensor array using the etching process.

Figure 4.45: Biasing field versus resonant and anti-resonant frequencies as well as relative signal strength for the $10 \mathrm{~mm}$ sensor element.

Figure 4.46: Biasing field versus resonant and anti-resonant frequencies as well as relative signal strength for the $8 \mathrm{~mm}$ sensor element.

Table 3.1: Pin functions and connection for the LCD module.

Table 3.2: Transferred data for the Control Register.

Table 3.3: Transferred data for the $F_{\text {start, }}$ low bits.

Table 3.4: Transferred data for the $F_{\text {start }}$, high bits.

Table 3.5: Transferred data for the $\Delta F$, low bits.

Table 3.6: Transferred data for the $\Delta F$, high bits.

Table 3.7: Transferred data for the Number of Increments.

Table 3.8: Transferred data for the Burst Interval.

Table 3.9: Test port layout on the PCB. 


\section{Acknowledgements}

Firstly I would like to thank my supervisor Dr. Gideon Gouws for endless help throughout the project. Also thanks to electronics technicians Johnny McClymont, Tim Exley and Jason Edwards for providing technical help. Thanks to Alan Rennie and Manu Pouajen-Blakiston from the mechanical workshop for constructing a number of structures used in the project. Thanks to Dr. Thomas Borrmann for providing help with sensor coatings and David Flynn for assisting with the use of mounting waxes. Thanks also to Scotty Forbes for computer help.

Finally I would like to thank my parents for their support and encouragement. 


\begin{abstract}
This thesis describes the development of a prototype passive, wireless sensor system based on magnetoelastic sensor technology. The application of the sensor system is aimed at the measurement of humidity levels in sealed containers and structures, in particular the detection of moisture ingress in building structures. The system uses magnetoelastic sensor elements as is commonly used in commercial electronic article surveillance (EAS) systems for the prevention of shoplifting. Magnetoelastic Metglas $(\mathbb{R} 2826 \mathrm{MB}$ material is evaluated and it is shown that the application of a magnetic bias field of the appropriate magnitude is critical for optimum sensor performance. The sensors can be activated for the detection of water vapour by the application of an appropriate chemical interface layer. A polyvinyl alcohol (PVA) layer was found to produce a resonant frequency response of approximately $50 \mathrm{~Hz} / \mathrm{RH}(\%)$. The design and construction of a portable electronic sensor reader is described and it is shown that the reader can successfully interrogate the sensor inside sealed spaces at practical distances. The construction of a practical humidity sensor using a PVA coating and the humidity response of the sensor system is then calibrated using a commercial humidity sensor. The system showed good response over the $0-100 \%$ relative humidity range, with some non-linearity observed at high humidity levels. The completed sensor system was then evaluated for practical applications including measurements inside a sealed food container as well as inside a wall cavity. The results indicated that the constructed system was capable of measuring humidity with reasonable accuracy in such applications.
\end{abstract}




\section{Introduction}

Today many sensors and sensor systems are available off the shelf and allow measurement of a wide range of environmental parameters such as humidity, temperature, pressure or the concentration of various chemical species. However, nearly all these sensor systems suffer from two potential drawbacks. The first is that they are active sensors, meaning that they are continuously powered and thus needs a constant power source such as a battery. This leads to limitations in terms of battery lifetime or in sensor mobility if it is powered from a fixed source. A second drawback of conventional sensor systems is the fact that direct physical contact in the form of electrical conductors is normally needed between the sensor and readout electronics. This places constraints on the configuration between readout electronics and the sensor element and limits the mobility of the sensor system.

The aim of this work is now to develop a sensor system that will overcome the two limitations described above. The system should be passive so the sensors should not require its own power source and it should also allow remote readout of the sensors. Such sensor systems have in fact been in commercial use for many years in retail stores around the world in the form of electronic article surveillance (EAS) systems [1]. In these systems a passive sensor or tag is fixed to the retail item and if the item is removed from the store without payment, the tag is remotely detected at the store exit by a reader system. Such EAS systems are then simple 1-bit sensors, simply providing a "present" or "not present" signal. However, it has been shown that the same can be applied to create passive, remotely readable environmental sensor systems [2].

It is envisaged that a passive remote sensor system will have many applications, particularly in the monitoring of sealed spaces over extended periods of time. The passive nature of the sensor will enable it to be enclosed in the target environment for long periods of time, while the remote readout capability will enable the sensor signal to be acquired without opening the sensor environment. One possible application may be the monitoring of sealed food or chemical containers. 
An application that is considered particularly interesting is the monitoring of environmental conditions in sealed building spaces and cavities. In recent times the effect of water ingress in New Zealand building structures due sub-standard materials and inappropriate construction methods has been regularly in the news [3] [4]. Once moisture has penetrated a building structure, it can become trapped and lead severe deterioration of the structure as shown in Fig. 1.1, particularly when poor quality building materials had been used. The Auckland City Council expects to receive approximately a 1000 claims costing from $\$ 350$ to $\$ 500$ million in the next decade due to leaky building structures. In addition, high moisture levels in homes can lead to severe health problems such as the presence of fungi in the home or increased risks of asthma or other breathing related diseases.

The ability to regularly check for the ingress of moisture in sealed building cavities using a passive, remote sensor system can then be a valuable tool in the early detection of leaky building structures.

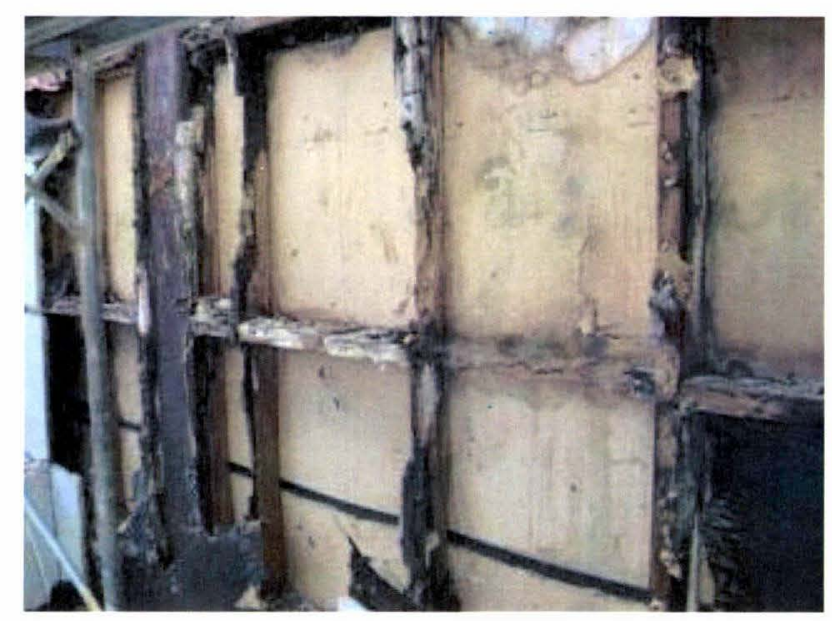

Figure 1.1: The damage resulting due to water penetration of a building structure and the subsequent deterioration of sub-standard building material.

The broad goal of this project was then the development of a prototype passive remote sensor system and the proof of concept application of the system for the monitoring of moisture in building structures. In a previous project at Victoria University [5], the use of magnetoelastic materials for passive sensors in laboratory scale applications had been demonstrated. 
This project will now aim to extend this work, with the following particular milestones:

- An evaluation of magnetoelastic materials and the properties of these sensors and materials important for environmental sensor applications.

- The design of a handheld reader that will enable practical, real-world applications of these sensors.

- The design of practical sensor elements for the detection of water vapour.

- The prototyping of such a sensor system in a practical application, simulating water ingress in a building structure.

The appropriate background theory to magnetoelastic materials and sensors is first discussed in Chapter 2. A portable, handheld sensor reader was developed which enabled these sensors to be remotely interrogated and the development of this reader is described in Chapter 3. This is followed in Chapter 4 by the main results on the development of sensors for water vapour, the evaluation of these sensors and the application of the sensors and the sensor reader to building structures. A summary and conclusions from the work is then presented in Chapter 5 . 


\section{Background}

\subsection{Introduction}

This chapter provides an introduction to the theory of passive, wireless sensors. As the operation of these sensors is based on the electronic article surveillance (EAS) technology this technology will first be reviewed in Section 2.2.

The use of magnetoelastic tags in EAS applications are then discussed in Section 2.3. This section also contains an introduction to the magnetic properties of magnetoelastic materials as these determine the performance of these sensors. Some of the previous work by Smith [5] on sensor properties at the School of Physical and Chemical Sciences (SCPS) will also be presented. A short literature survey on detection methods and sensor applications of magnetoelastic sensors will conclude Section 2.3 .

\subsection{Electronic Article Surveillance}

Shoplifting is a serious problem in many countries with retailers losing billions of dollars worth of merchandise per year worldwide [6]. The two most common methods employed to reduce theft in stores include closed-circuit television (CCTV) and antitheft tags. The two methods are often used simultaneously, and experts believe that together they are the most effective means to reduce theft [7].

CCTV involves the use of video cameras which are used to record or monitor customer activity. This can identify shoppers who are acting suspiciously or are committing a crime, as well as being able to capture their appearance or theft patterns. The resulting footage can be used as evidence or to help identify the culprit in advertisements.

Antitheft tags come in two major forms, either reusable tags or disposable tags, which are attached to the items in the store. Reusable tags are non-electronic tags, and some examples are shown in Fig. 2.1. Such tags are attached to the items in the store and 
cannot be removed without special tools available to the cashier, called "alligators" [7]. They deter shoplifters by visibly sending a message that they are secured and cannot be removed. Some tags are fluid (such as ink) filled and once they are illegally removed, the item (such as clothing) is ruined and is no longer valuable.

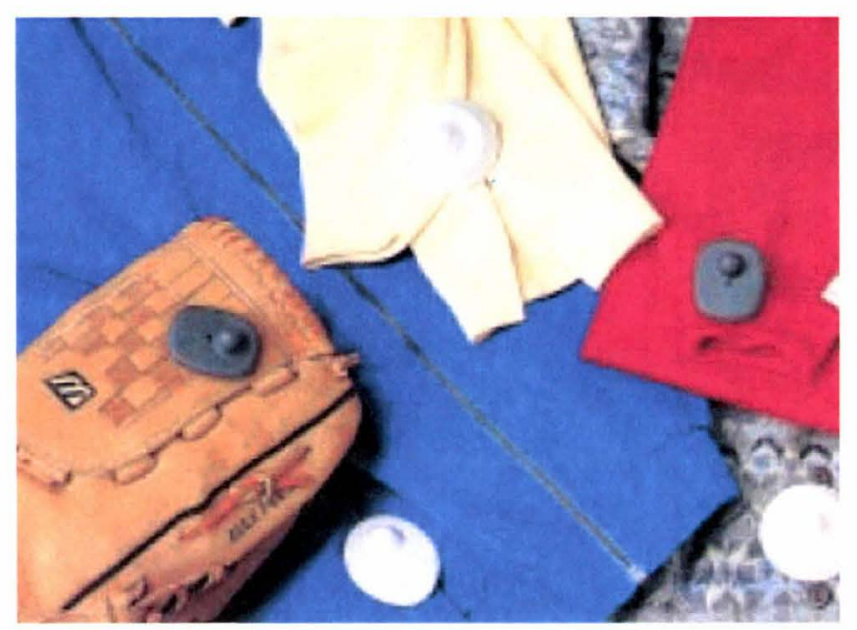

Figure 2.1: Reusable antitheft tags attached to store items.

Disposable tags refer to electronic article surveillance (EAS) tags, which are the technology on which our wireless sensors are based. This type of tag responds to the detector pedestals placed near the exit of the store, and unlike the reusable tags, they cause an alarm if the tag is not removed from the item or deactivated by a member of staff at checkout.

There are three major types of EAS system in use today: radio-frequency (RF) systems, harmonic (also known as electromagnetic) systems and acousto-magnetic (also known as magnetoelastic) systems. The most common configuration of EAS systems utilises a pair of detector pedestals, one being the excitation pedestal, producing an electromagnetic signal to excite the tag, and the other to detect the tag's response. Fig. 2.2 shows such a configuration. 


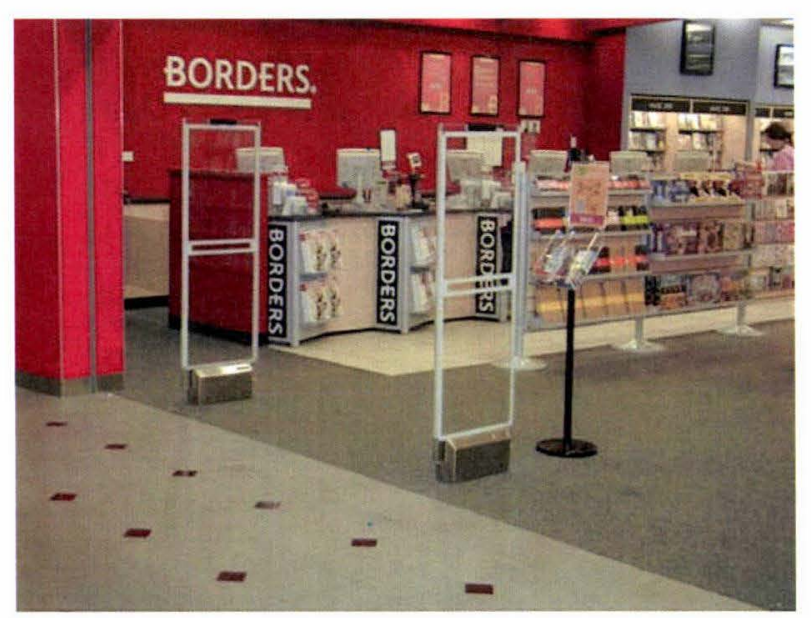

Figure 2.2: EAS detectors found at the entrance of a major bookstore.

RF and magnetoelastic EAS systems operate in a similar manner. The tag is excited at its characteristic resonance (or resonant) frequency after which the tag magnetically rings down as shown in Fig. 2.3. During this ring-down it emits its own AC magnetic flux for a relatively long period (a few milliseconds).

The magnetic signals from the tags produce small voltage signals in the detector antennas and from this signal the presence of the tag is determined. Because such tags only respond to a single predetermined frequency and responds in a predetermined way, the detector can identify the presence of the tag and activate the alarm.

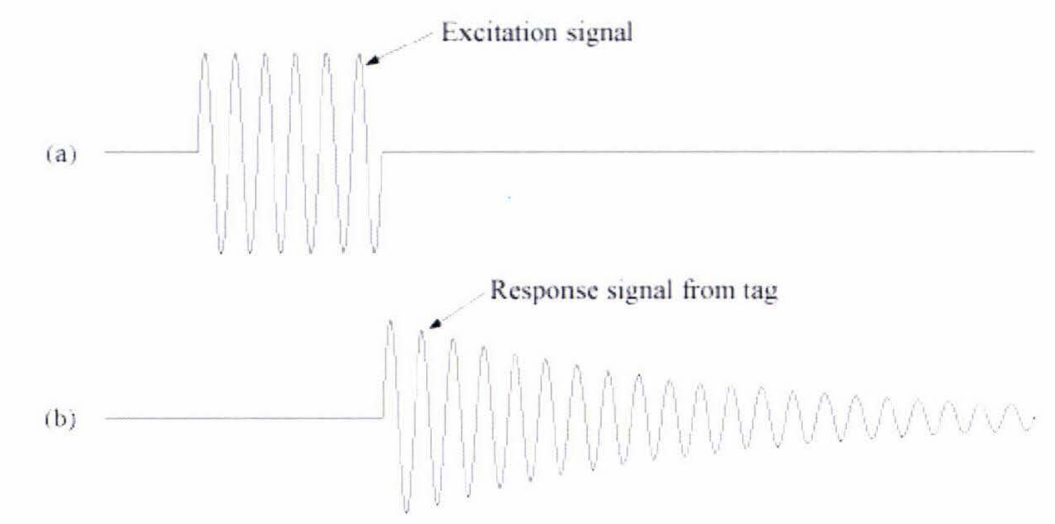

Figure 2.3: If the excitation signal (a) is of the same frequency as the tag's resonant frequency, the tag's response (b) will be long and be of expected frequency and length.

Harmonic systems are widely used in Europe [1] and in New Zealand they are often used in libraries. Such systems have tags which rely on a magnetically soft metallic 
strip together with a series of semi-hard magnets, which are initially demagnetised (armed). When the item is put through the detector gates the alarm is activated, as the low frequency excitation electromagnetic field given off by the detector causes the soft metallic strip to magnetically saturate, causing a periodic "spike" in the detector antenna, which are identified as the presence of the tag.

When the item is legally checked out, the semi-hard magnets are magnetised (disarmed), locally saturating the soft metallic strip, effectively breaking it down into smaller pieces. This prevents the strip from saturating by the excitation signal, making it no longer respond to the detector.

\subsection{Magnetoelastic Tags}

Magnetoelastic tags rely on a magnetostrictive alloy ribbon as the resonator. These magnetoelastic materials efficiently convert magnetic energy into mechanical oscillations (i.e. they have good magnetoelastic coupling), and vibrate longitudinally in response to an externally applied $\mathrm{AC}$ magnetic field through an effect called magnetostriction. This vibration causes mechanical stresses within the material, changing its magnetisation and induces a small voltage in a nearby coil [1]. The size of oscillation has its largest amplitude and is longest when the external AC magnetic field is equal to the ribbon's resonant frequency, a value which is mainly determined by the length of the ribbon.

\subsubsection{Antitheft Applications}

A commercial magnetoelastic tag used for EAS is shown in Fig. 2.4. Such tags typically contain two ribbons of the resonator material which are shaped to resonate at about $58 \mathrm{kHz}$ - having two strips increases the signal strength. Placed together with the magnetoelastic strips is a magnetically semi-hard magnet for biasing and deactivation purposes [1].

Before being placed in the casing the magnetoelastic strips are often placed for a certain time in strong magnetic fields and high temperature (called annealing) to enhance the material's properties [1] [8]. The resonator strips are then loosely placed 
inside the casing and is able to vibrate freely, while the semi-hard strip is encased inside an air filled case, which acts to reduce damping on the resonator strips.

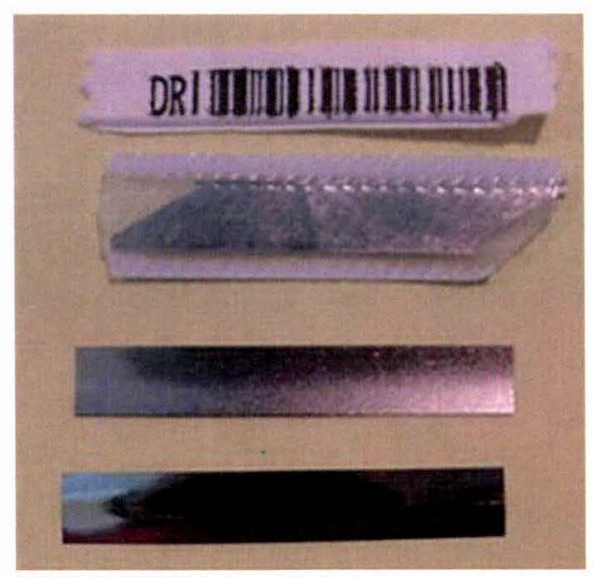

Figure 2.4: A commercial magnetoelastic EAS tag, showing two magnetoelastic strips and a biasing magnet inside an air filled case, shaped like a parallelogram.

The excitation antenna produces periodic tonal bursts at the same frequency as the resonant frequency of the tags, and if the tag is in its interrogation zone the strips vibrate longitudinally by magnetostriction (see Section 2.3.2.5). The vibrations produce mechanical stresses within the material, causing magnetisation oscillations which induce a corresponding voltage in the receiver antenna [1]. When the excitation tone is over, the vibration amplitude rings down exponentially (as shown in Fig. 2.3), and the detection circuitry in the detector pedestal analyses this voltage signal, checking whether it is the correct frequency, repetition rate and amplitude. If these criteria are met, the alarm is activated [7].

The semi-hard magnet is a rectangle or parallelogram shaped strip of magnetic alloy, such as Arnokrome from Arnold Technology or Semivac from Vacuumschmelze $\mathrm{GmbH}$ [9]. This semi-hard magnet applies an appropriate DC magnetic field (typically a few Oe) to the magnetoelastic strips which offsets the magnetic anisotropy (see Section 2.3.2.4) in the material [1]. This has the effect of increasing the amplitude of oscillation substantially, as well as changing the resonant frequency due to the $\Delta E$ effect (see Section 2.3.2.6). The magnet is semi-hard and so it can be demagnetised easily during checkout with an electromagnet. This causes the vibration to become small enough that it can no longer be detected by the pedestals. 


\subsubsection{Magnetoelastic Materials}

In conventional metals, liquid-solid equilibrium is reached when the melt solidifies into its lowest energy state giving a crystalline lattice structure [10]. However magnetoelastic materials, such as Metglas $2826 \mathrm{MB}$ used in this project are produced in such a way that gives the material little time to crystallize. One way this is achieved is by rapid melt quenching, illustrated in Fig. 2.5. The melt is squirted onto a cooling wheel in a vacuum, spinning at up to $100 \mathrm{~km} / \mathrm{h}$ [6]. This gives cooling rates of up to $100{ }^{\circ} \mathrm{C}$ per second and due to this high cooling rate, the atoms in the material do not form a crystalline structure but form a glass-like, tightly-packed, disordered state; hence they are also called "glassy" or amorphous (non-crystalline) metals.
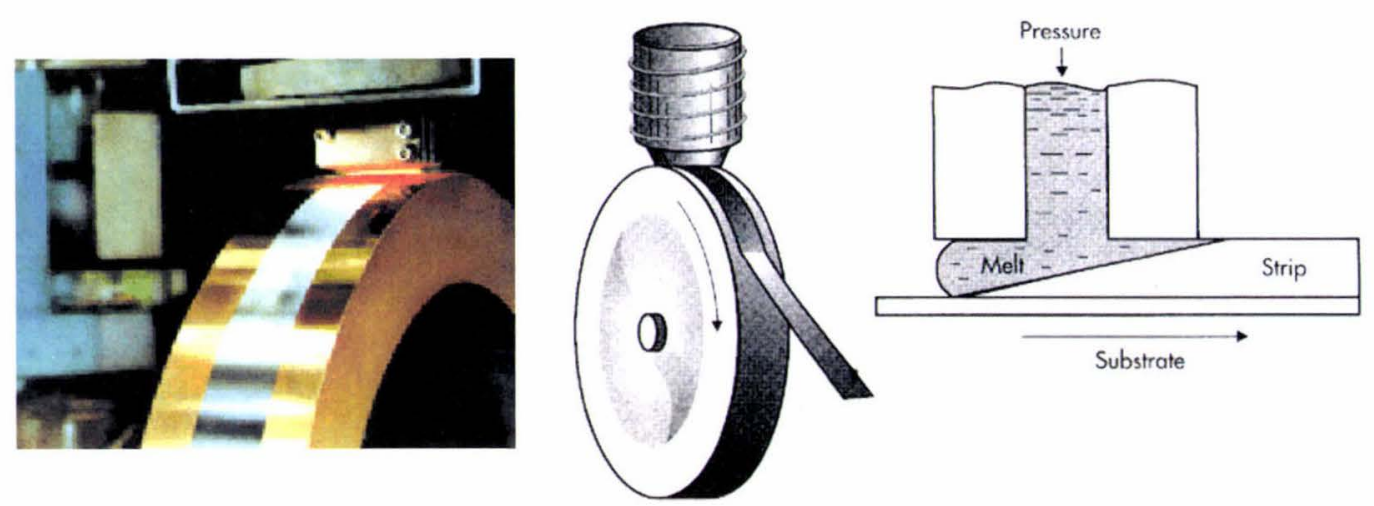

Figure 2.5: Production of the amorphous metal ribbons with a cooling wheel.

The melt is typically consisting of ferromagnetic elements iron, nickel, cobalt and in smaller amounts "glass forming" elements such as silicon and boron [11]. Metglas ${ }^{\circledR}$ $2826 \mathrm{MB}$ used in this project is made from 33 - 48 weight \% iron, 41 - 55 weight $\%$ nickel, $0-9.5$ weight $\%$ molybdenum, and $2.8-5 \%$ boron. The nominal atomic composition is $\mathrm{Fe}_{40} \mathrm{Ni}_{38} \mathrm{Mo}_{4} \mathrm{~B}_{18}$ [12] [13].

Because the cooling wheel only makes contact to one side of the material, the side which makes contact with the wheel is rough, while the other is smooth [14], as shown in Fig. 2.6. It will be shown in later chapters that having rough sides is useful when applying chemically reactive coatings for sensor processing. 
Due to its structure, metallic glasses absorb less energy upon stress induced deformation through damping and return more by rebounding elastically to its initial shape. This gives such materials high strength, hardness, toughness and elasticity [10] as well as resistance to corrosion.

These materials can also be very magnetically soft and have relatively high electrical resistance, meaning it can be easily controlled through an external magnetic field with little eddy current loss. Such electrical and magnetic qualities mean they are well suited for passive sensor applications. For example, Metglas 2826MB has electrical resistivity of $1.38 \mu \Omega-m$ [12], which equals a resistance of about $5 \Omega / m$ for strips $30 \mu \mathrm{m}$ thick and $10 \mathrm{~mm}$ wide. Standard metals such as copper have resistivity values two orders of magnitude lower (copper has a resistivity of about $17 \mathrm{n} \Omega-\mathrm{m}$ [15]).
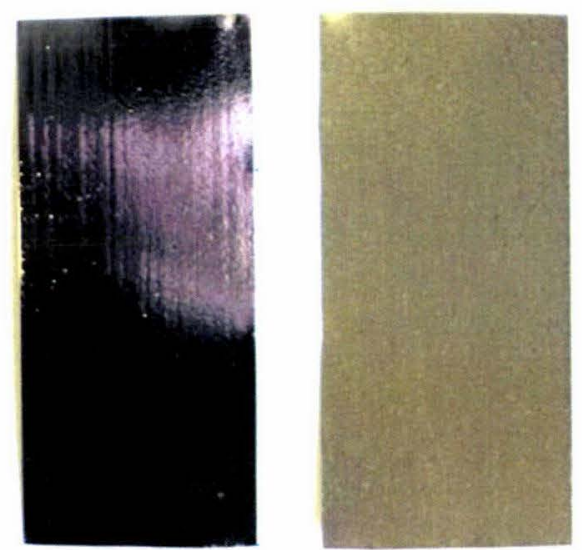

Figure 2.6: Smooth (left) and rough (right) sides of Metglas 2826MB ribbons.

Due to their slow heat conduction, early amorphous metals were restricted to thin ribbons tens of $\mu \mathrm{m}$ thick. However, active research in the field has resulted in materials with critical casting thicknesses in the order of $\mathrm{cm}$, giving rise to bulk metallic glasses [10].

Due to this, such materials have moved from sensors and EAS applications into a wide variety of applications. A major company named LiquidMetal Technologies exists in the US [16], which develops bulk amorphous metal alloys for a range of fields. 
Examples include the military, where the company is working with the US Department of Defence to develop lower cost, high strength and lower weight kinetic energy penetrator (KEP) ammunition. In electronics, LiquidMetal produce light weight, durable and cases for electronic products such as mobile phones, liquid crystal displays (LCDs) and USB drives. In medicine, amorphous metal alloys are used in surgical blades and reconstructive devices. LiquidMetal are also working with NASA to develop amorphous metals for space projects and its products have been onboard spacecraft for experimentation. Other applications include sports equipment such as golf club heads, tennis racquets and baseball bats, jewellery and mechanical products such as hinges.

The advantages of high strength, hardness, strength-to-weight ratio, elasticity and corrosion resistance offered by amorphous metals are the main selling points of these products. Fig. 2.7 shows some examples.

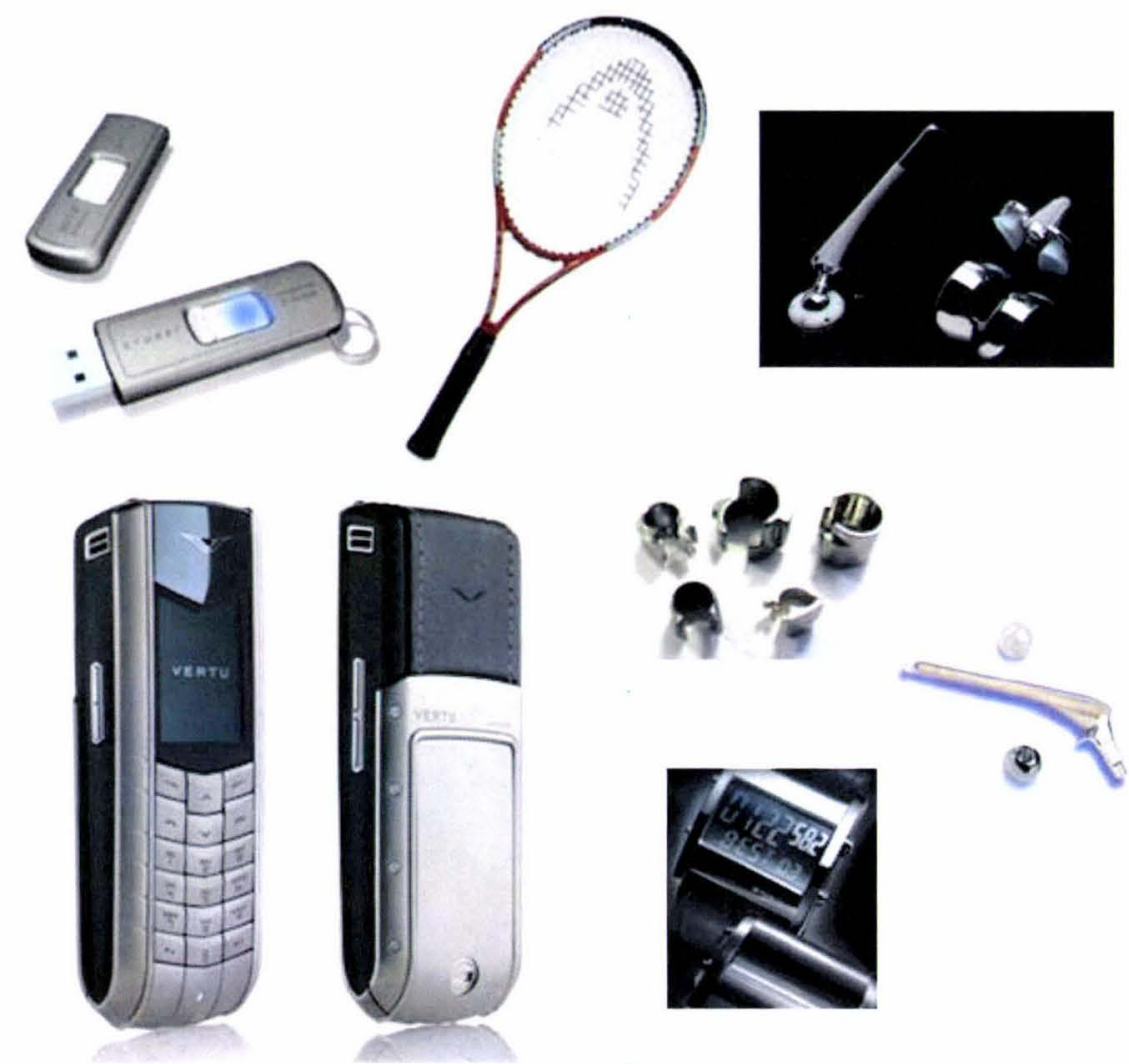

Figure 2.7: Examples of products made from amorphous metal alloys; cases for USB drives and mobile phones, tennis racquets, hinges, medical instruments and jewellery. 


\subsubsection{Ferromagnetism}

Ferromagnetic elements such as iron, cobalt or nickel have electron spins of one atom interacting with neighbouring atoms, causing alignment of magnetic dipole moments. This quantum mechanical effect called exchange coupling, give such materials permanent magnetism [15] and can remain magnetised for a long time after the external field is removed. If the temperature of the material is raised beyond its Curie temperature $\left(\mathrm{T}_{\mathrm{C}}\right)$, the exchange coupling ceases and becomes paramagnetic [17] [18]. For Metglas $2826 \mathrm{MB}$ this temperature is $353^{\circ} \mathrm{C}$ [12], which is high enough that it will not be encountered in normal circumstances.

\subsubsection{Domain Theory}

Exchange coupling produces strong alignment of adjacent atomic dipoles in ferromagnetic materials, given it is under the Curie temperature. Thus such materials can be magnetised even in very weak fields. However, we do not find that all ferromagnetic materials are naturally magnetised to its maximum value (saturation). This was explained by the domain theory, first postulated by Weiss in 1907 [19].

Ferromagnetic materials are known to be composed of regions where atomic dipoles are almost perfectly aligned called magnetic domains. Such domains act as tiny magnets inside the material, and the orientations of the domains determine the material's net magnetisation. When such domains are oriented in random directions, the magnetic fields of the domains cancel and the net magnetic field is zero. If stresses or external magnetic fields are applied to the material, the domains will tend to change in orientation, causing the material to become magnetised, with some of the domains becoming oriented in the same direction. Magnetic saturation is reached when all the domains in the material are pointing in the same direction and thus cannot be magnetised further.

When a ferromagnetic material is magnetised, surface charges appear at the ends of the material, producing a secondary source of magnetic field (the demagnetising field). Domains are formed due to the energy considerations in the surface charge distribution, called the magnetostatic energy. The demagnetising field is equal to the 
volume integral of the field over all space. If a material is composed of a single domain, the demagnetising field would be large, and would thus have large magnetostatic energy. This energy can be reduced by approximately $1 / \mathrm{N}$ if the magnetisation splits into $\mathrm{N}$ number of opposite domains, as this has the effect of reducing the spatial extent of the demagnetising field, shown Fig. 2.8.

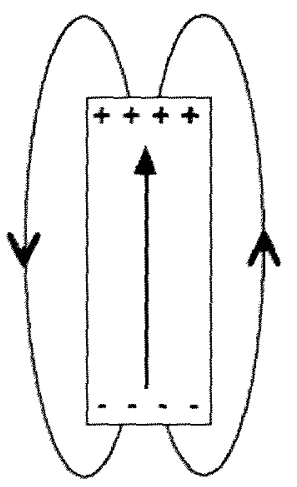

Single Domain
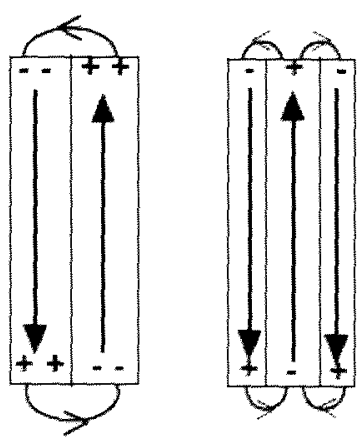

Multidomain

Figure 2.8: Reduction of magnetostatic energy through domain formation.

The number of domains is limited by the size of the particle, because the transition region between domains (called the domain wall or Bloch wall) has a finite width, which is determined by exchange and magnetocrystalline energies [18] [20]. Fig. 2.9 shows the domains of an amorphous metal ribbon, taken using a scanning electron microscope (SEM). The ribbon is demagnetised, and arrows show the directions of each domain.

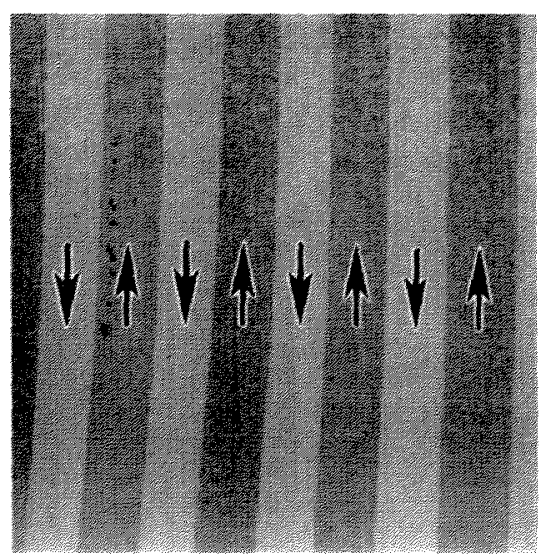

Figure 2.9: Magnetic domains in a demagnetised amorphous metallic ribbon, taken with a scanning electron microscope (SEM) [11]. 


\subsubsection{Hysteresis}

Ferromagnetic materials have the property in which it can retain some of the external field it was subjected to even after it has been removed. This effect is known as hysteresis and the effect can be summarised with the hysteresis loop of Fig. 2.10 [18].

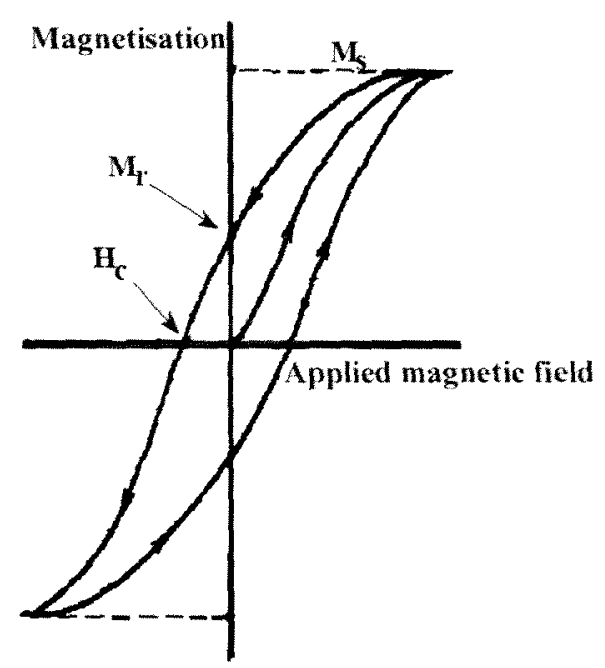

Figure 2.10: Hysteresis loop for a ferromagnetic material showing saturation $\left(M_{s}\right)$, remanence $\left(M_{r}\right)$ and coercivity $\left(H_{c}\right)$.

The material is initially at zero magnetisation and in zero field at the origin, and as the applied field is increased the material's magnetisation increases until its reaches saturation magnetisation $\left(\mathrm{M}_{\mathrm{s}}\right)$. If the applied field is then reduced to zero, the material keeps some of its saturation magnetisation, and this point is known as saturation remanence $\left(\mathrm{M}_{\mathrm{r}}\right)$. The opposite magnetic field required to remove $\mathrm{M}_{\mathrm{r}}$ is defined as the material's coercivity (or coercive field) $H_{c}$ and if this is large, the material is said to be magnetically hard, being more difficult to demagnetise and if small it is called magnetically soft being easy to demagnetise.

The hysteresis loop of Metglas 2826MB is shown in Fig. 2.11 for both as-cast (as prepared) and annealed samples. Longitudinal annealing is achieved by exposing the material to the magnetic field parallel to the strip axis, while transverse annealing is achieved by aligning the magnetic field perpendicular to the ribbon axis of the strip. 
For all cases the coercivity is very low being under $0.5 \mathrm{Oe}$, indicating that it is very magnetically soft.

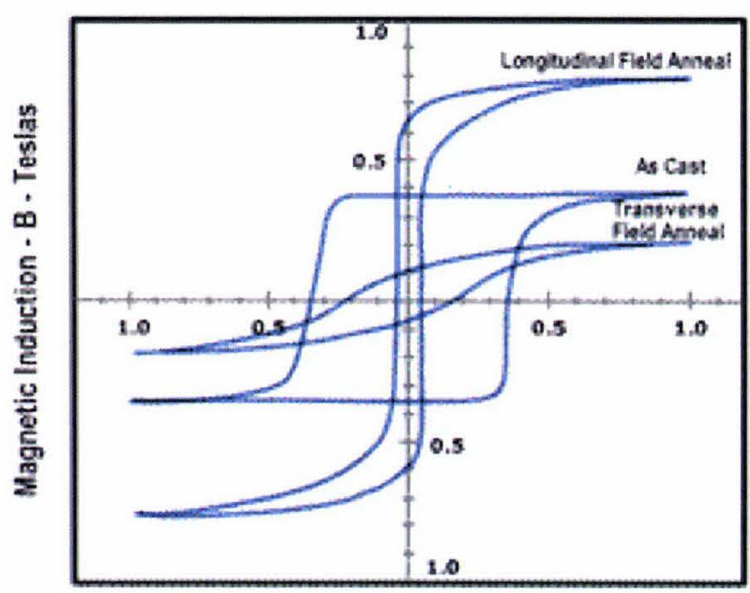

Magnetizing Force - H - OERSTEDS

Figure 2.11: Hysteresis loop of Metglas 2826MB showing plots of both as-cast and annealed samples [12].

\subsubsection{Magnetic Anisotropy}

Magnetic anisotropy is defined as the dependence of magnetic properties in certain directions, observed in ferromagnetic and ferrimagnetic materials. Low anisotropy implies that the material can be magnetised more easily, requiring less energy. There are three main types of anisotropy; magnetocrystalline anisotropy, shape anisotropy, and stress anisotropy. Magnetic anisotropy affects the shape of hysteresis loops, and controls coercivity and remanence values [18] [20].

Magnetocrystalline anisotropy is an intrinsic property of crystalline materials in which certain directions require less energy to magnetise in that direction. Such directions are called easy axes, and a magnetisation curve for magnetite, which has a crystalline structure, is shown as an example in Fig. 2.12.

Amorphous metals such as Metglas 2826MB do not have a crystalline structure and thus have no magnetocrystalline anisotropy. Since magnetocrystalline anisotropy is the dominant form of anisotropy in materials, this implies that magnetoelastic materials have very low anisotropy compared to crystalline materials. 


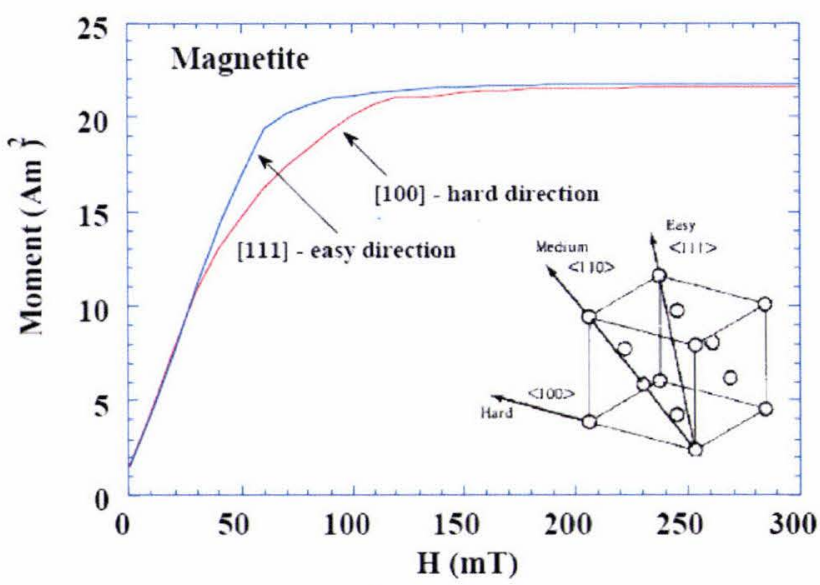

Figure 2.12: Magnetocrystalline anisotropy of magnetite [18].

When a ferromagnetic material is placed inside a magnetic field, surface charges appear near the ends of the material creating poles antiparallel to the external field, as shown in Fig. 2.13. This demagnetising field opposes the external field meaning that the net magnetic field inside the material is lower than the actual applied field [18]. The demagnetising field $H_{d}$ is calculated as [20]:

$$
H_{d}=\frac{N M}{\mu_{0}}
$$

where $M$ is the magnetisation of the sample, $\mu_{0}$ is the permeability of vacuum and $N$ is the demagnetising factor, which depends on the shape of the sample. For example, if a long thin needle is magnetised along its length axis, the demagnetising field would be small compared to other shorter axes, and thus would create an easy axis along the long axis. Spherical objects, because all axes are the same length, do not have this effect.

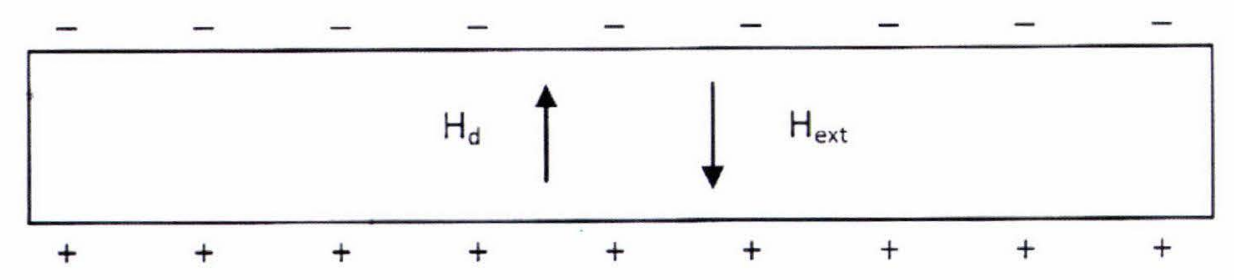

Figure 2.13: Schematic showing external field $\mathrm{H}_{\mathrm{ext}}$ and demagnetising field $\mathrm{H}_{\mathrm{d}}$ in a thin metal plate. 
This effect is called shape anisotropy, and it introduces some design issues in the shape of the sensor strip. If the aspect ratio (the ratio between length and width) is reduced, the effect of the demagnetising field would be larger and thus would require a larger DC biasing field to optimise its performance. Previous studies [5] showed that as the aspect ratio is increased, the DC biasing field required decreases exponentially, shown Fig. 2.14.

The Metglas ribbons used in this project had a standard width of $13 \mathrm{~mm}$, which are available as long strips from Metglas Inc. [12]. These were cut to constant lengths of $40 \mathrm{~mm}$, which gives an aspect ratio of about 3 .

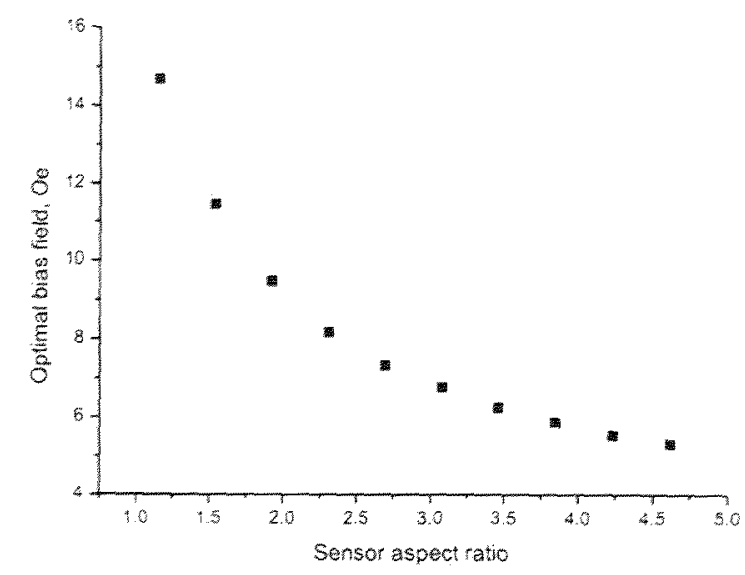

Figure 2.14: Sensor aspect ratio versus optimal biasing field [5].

Applied or residual stresses can also cause anisotropies in materials. This stress anisotropy is due to magnetostriction (see Section 2.3.2.5) and in as-cast amorphous ribbons there are always internal stresses present due to inhomogeneities in cooling rates when quenching the melt. This leaves a complex pattern of residual stresses within the material [14] [21], and the domains are randomly oriented.

In crystalline materials this effect is small compared to magnetocrystalline anisotropy, but in magnetoelastic materials this can be significant, and such effects may be controlled by annealing treatments, and/or by using a DC bias field as shown in the EAS tags of Fig. 2.4. These have the effect of reducing the anisotropy by aligning the magnetic domains within the material. In this project, the Metglas $2826 \mathrm{MB}$ strips were used as-cast without annealing for simplicity. 


\subsubsection{Magnetostriction}

The orientation of domains within the material can change due to magnetisation, and this can cause the material to physically change in size. This effect is called magnetostriction, and this effect is observed when a transformer is turned on, one hears "humming", where the transformer core physically vibrates at twice the mains frequency [22]. For transformer cores however, magnetostriction is not a desirable effect, as it results in energy loss (called hysteresis loss) within the core.

Unless otherwise stated, magnetostriction refers to the Joule effect or Joule magnetostriction. This is defined as the fractional change in the length of the material when magnetised [23]:

$$
\lambda=\frac{\Delta L}{L}
$$

where $\Delta L$ is the change in length between the original length and the length when magnetised, and $L$ is the original length. If the material expands when magnetised, the material is said to exhibit positive magnetostriction, while a contraction is referred to as negative magnetostriction. This effect is illustrated in Fig. 2.15, where a material exhibiting positive magnetostriction is put under a longitudinal magnetic field $\mathrm{H}$.
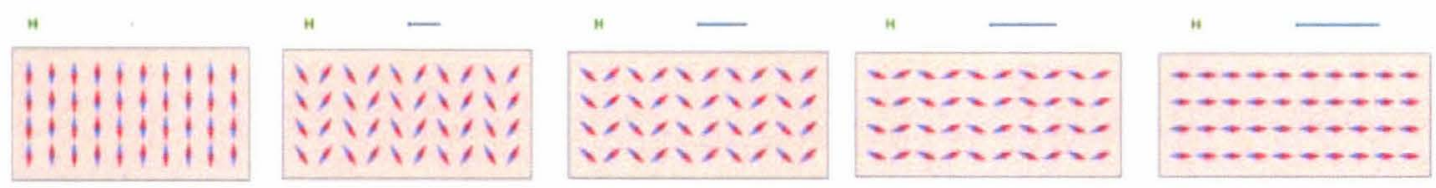

Figure 2.15: Schematic of a material exhibiting positive magnetostriction in a magnetic field, increased from left to right.

Magnetostriction of a material is commonly defined with saturation magnetostriction $\left(\lambda_{\mathrm{s}}\right)$, which defines the fractional change in length of the material between zero and saturation magnetisation [22] [23]. For Metglas 2826MB, the saturation magnetostriction is specified as about $12 \times 10^{-6}$ [13] . 
When magnetised in one direction the material expands, causing stresses within the material. These stresses can cause an easy axis to be created and this contribution to anisotropy is called the Villari effect [24]. In sensor and EAS applications the magnetoelastic strip is placed in an oscillating magnetic field, and therefore the interplay of the Joule and Villari effects are exploited.

\subsubsection{6 $\Delta \mathrm{E}$ Effect}

Young's modulus (also known as elastic modulus) of a material describes the stiffness of the material and is defined as the ratio of the material's stress and strain [25]:

$$
E=\frac{F / A}{\Delta L / L}
$$

where $F$ is the force experienced by the material, $A$ is the area of material, $D L$ is the change in length of material due to the force and $L$ is the length of material with no applied force. For Metglas $2826 \mathrm{MB}, E$ is between 100 and $110 \mathrm{GPa}$ [12].

The change in Young's modulus of a material due to magnetisation is known as the $\Delta E$ effect. This is defined as [26] [27]:

$$
\frac{\Delta E}{E_{S}}=\frac{E_{S}-E_{M}}{E_{S}}
$$

Where $E_{M}$ is Young's modulus at constant magnetisation, and $E_{S}$ is Young's modulus in field $\mathrm{H}$. The $\Delta \mathrm{E}$ effect is a consequence of magnetostriction in which the orientation of magnetic domains changes due to stress. As Fig. 2.16 shows, the strain in ferromagnetic materials is greater than in non-magnetic materials. This is due to the fact that the effective strain on the material is the sum of mechanical strain and magnetostrictive strain [28]:

$$
\varepsilon=\varepsilon_{S}+\varepsilon_{H}
$$


The $\Delta \mathrm{E}$ effect has important consequences in magnetoelastic tags. Because the DC biasing field on the material affects the elasticity of the material, the resonant frequency of the tag can also shift; in EAS systems this effect is not desirable, as small changes in magnetic field, caused by factors such as scattering of the biasing magnet or alignment to Earth's magnetic field could potentially render the tag useless. This is minimised by choosing resonator strips with relatively low magnetostriction and relatively high anisotropy [1].

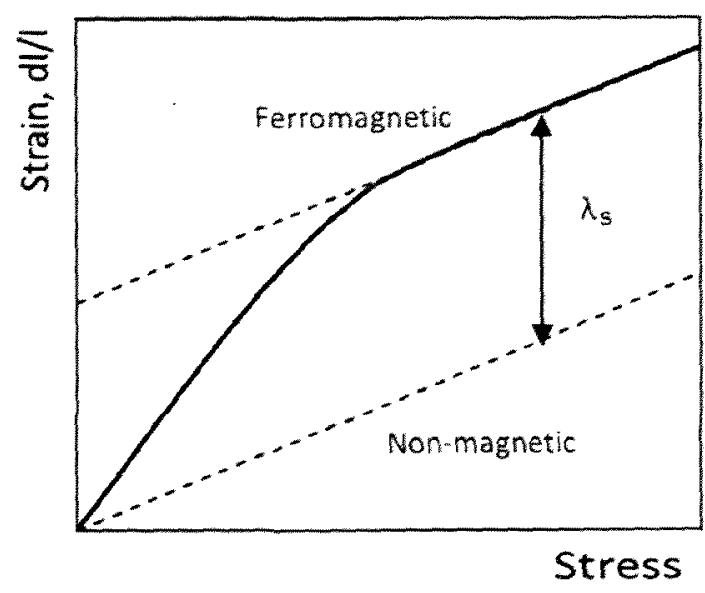

Figure 2.16: Stress-strain curves of ferromagnetic and non-magnetic materials, also showing the saturation magnetostriction $\lambda_{s}[23]$.

\subsubsection{Modelling the Material}

The vibration of a magnetoelastic sensor with length parallel to the $\mathrm{x}$-axis, excited by an $\mathrm{AC}$ magnetic field along the $\mathrm{x}$-axis can be modelled using the equation of motion as [26] [29]:

$$
\frac{\partial^{2} u_{x}}{\partial t^{2}}=\frac{E}{\rho\left(1-\sigma^{2}\right)} \frac{\partial^{2} u_{x}}{\partial x^{2}}
$$

Where $u_{x}$ is the local displacement along the $\mathrm{x}$-axis which is taken to be the long dimension of the ribbon, $\rho$ is the density of sensor, $E$ is the Young's modulus of sensor and $\sigma$ is Poisson's ratio of the sensor. 
Solving the differential equation yields the equation for the sensor's resonant frequency:

$$
f_{n}=\frac{n}{2 L} \sqrt{\frac{E}{\rho\left(1-\sigma^{2}\right)}}
$$

Where $L$ is the length of sample, and $n$ implies the $n^{\text {th }}$ harmonic. The fundamental $(n=1)$ frequency is the largest signal, and this is the frequency of interest. The dependence on Young's modulus of the material to its motion explains the reason behind the change in resonant frequency due to the $\Delta \mathrm{E}$ effect.

A simpler alternative formula for resonant frequency can be found by noting that since $f_{n}$ is proportional to $L^{-1}$, if the two are plotted against each other, the graph is a straight line. The experimentally observed variation in $f_{n}$ with $L$ is shown in Fig. 2.17, showing a dependence of $1963 \mathrm{~Hz}-\mathrm{m}$ [5].

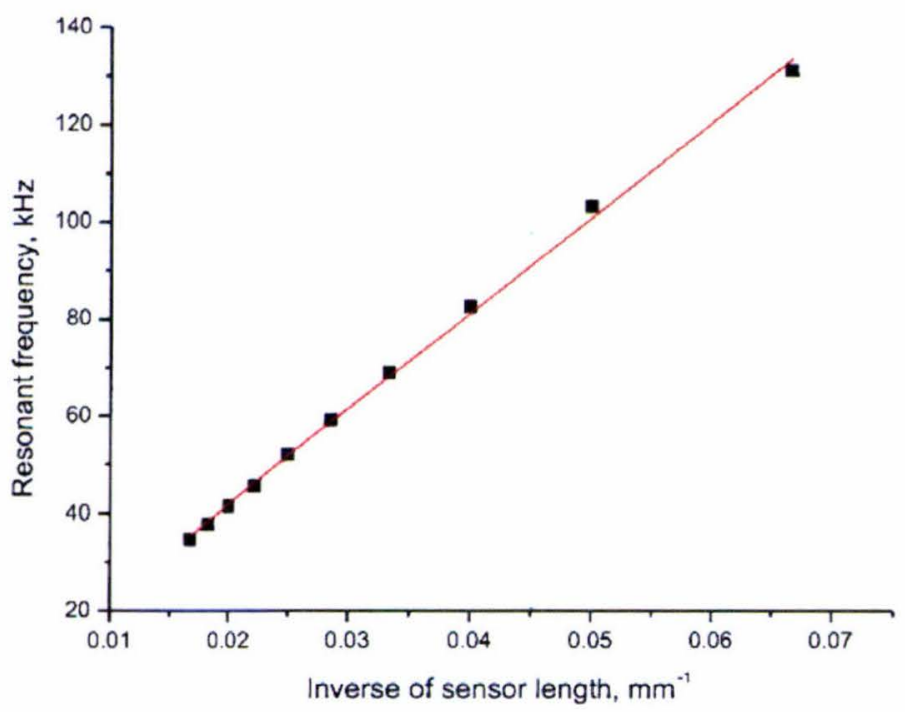

Figure 2.17: Inverse of sensor length versus resonant frequency [5].

\subsubsection{Mass Loading of Magnetoelastic Sensors}

Since the vibrating magnetoelastic strip can be modelled by a second order mechanical system, basic mechanical theory predicts that an increase in mass will decrease the frequency of oscillation. This is indeed the case, and this therefore means 
that if the mass of the strip can change due to the environmental parameter to be measured, the strip can be adapted for use as an environmental sensor by measuring the change in resonant frequency. This is achieved by coating the strip with a mass changing chemically reactive layer.

If a uniform coating is applied to the surface of the sensor strip, the density $\rho$ can be replaced as [26]:

$$
\rho=\frac{m+\Delta m}{A d}
$$

where $m$ is the mass of sensor, $\Delta m$ is the mass of the coating, $A$ is the surface area of the coating and $d$ is the thickness of sensor. By substituting this value into Eq. 2.6 and solving the equation gives the new equation for the resonant frequency:

$$
f_{\text {load }}=\frac{1}{2 L} \sqrt{\frac{1}{1+\Delta m / m} \frac{A d}{m} \frac{E}{\rho\left(1-\sigma^{2}\right)}}=f_{0} \sqrt{\frac{1}{1+\Delta m / m}}
$$

If the mass of the coating is much lower than the mass of the sensor strip, then the frequency shift can be approximated by:

$$
\Delta f=f_{\text {load }}-f_{0}=-f_{0} \frac{\Delta m}{2 m}
$$

Hence if the mass of the coating can change with changing environmental parameters, that parameter can be measured from the change in resonant frequency.

Previous work by Smith [5] was done to apply humidity responsive coatings to the Metglas strips. The work involved coating the strips with polypyrrole (PPy) and polyvinyl alcohol (PVA). The responses of PPy and PVA are shown graphically in Fig. 2.18 . 

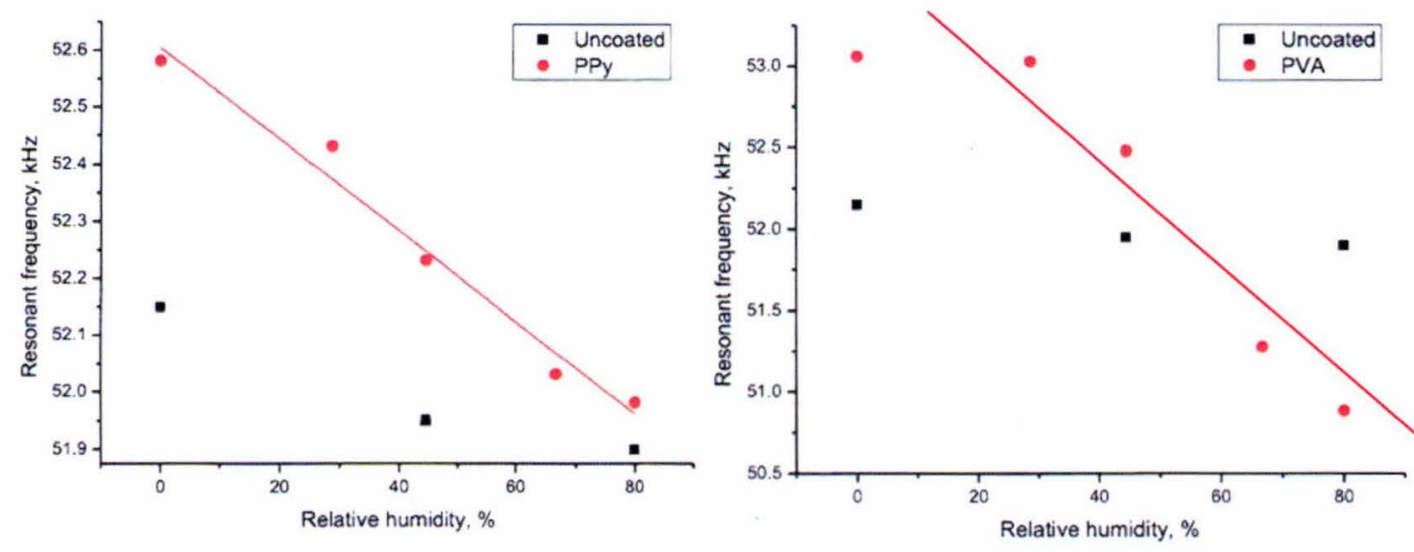

Figure 2.18: Previous results with PPy (left) and PVA (right) coated sensors [5].

PPy was found to have a roughly linear response with a sensitivity of $7.5 \mathrm{~Hz} / \mathrm{RH}(\%)$, with a response time of about 20 minutes, while PVA was found to have a more nonlinear response, but with a higher sensitivity of $30 \mathrm{~Hz} / \mathrm{RH}(\%)$, and a longer response time of about 30 minutes.

\subsubsection{Temperature Dependence}

If the $\Delta \mathrm{E}$ effect is extended to include temperature dependence, the formula for Young's modulus of Eq. 2.4 becomes [26]:

$$
\frac{\Delta E}{E_{S}}=\frac{E_{S}-E_{M}}{E_{S}}=\frac{9 \lambda_{S}^{2}(T) E_{S}(T) H^{2}}{M_{S}(T) H_{k \sigma}^{3}(T)}
$$

where $T$ is the absolute temperature, $\lambda_{S}$ is the magnetostriction, $H$ is the applied field, $M_{S}$ is the saturation magnetisation, $H_{k \sigma}$ is the anisotropy field when the sensor is under a longitudinal stress $\sigma, E_{M}$ is Young's modulus at constant magnetisation, and $E_{S}$ is Young's modulus in field $H$.

If Eq. 2.11 is substituted into Eq. 2.7, the resonant frequency $f_{0}$ with temperature dependence is obtained:

$$
f_{0}(T, H)=\frac{1}{2 L(T)} \sqrt{\frac{E_{S}(T)}{\rho_{S}(T)}}\left(1+\frac{9 \lambda_{S}^{2}(T) H^{2} E_{S}(T)}{M_{S}(T) H_{k \sigma}^{3}(T)}\right)^{-1 / 2}
$$


Eq. 2.12 implies that by applying an appropriate DC biasing field to the sensor, it can be made to be temperature dependant or independent. Previous work by Smith [5] was done to determine the temperature dependence of $30 \mathrm{~mm}$ long Metglas 2826MB strips. The results are summarised in Fig. 2.19.

The work of [5] showed that the strips have temperature dependence of $-10 \mathrm{~Hz} /{ }^{\circ} \mathrm{C}$ in a low bias field of 1 Oe. This field does not offset the magnetic anisotropy of the material and Young's modulus of the sample reduces to a purely mechanical elasticity, which decreases linearly with temperature.

In a high bias field of $13.01 \mathrm{Oe}$, where the material is magnetically saturated, temperature dependence of $-13 \mathrm{~Hz} /{ }^{\circ} \mathrm{C}$ was measured. Here the material's magnetostrictivity is minimal, and again the temperature dependence arises from mechanical means.

From [5] it was found that implementing a practical temperature sensor from Metglas $2826 \mathrm{MB}$ strips may be difficult, as it was found that Metglas strips in as-cast state has relatively small temperature dependence, and require either a very high or very low biasing field to obtain a decent amount of resonant frequency shift.

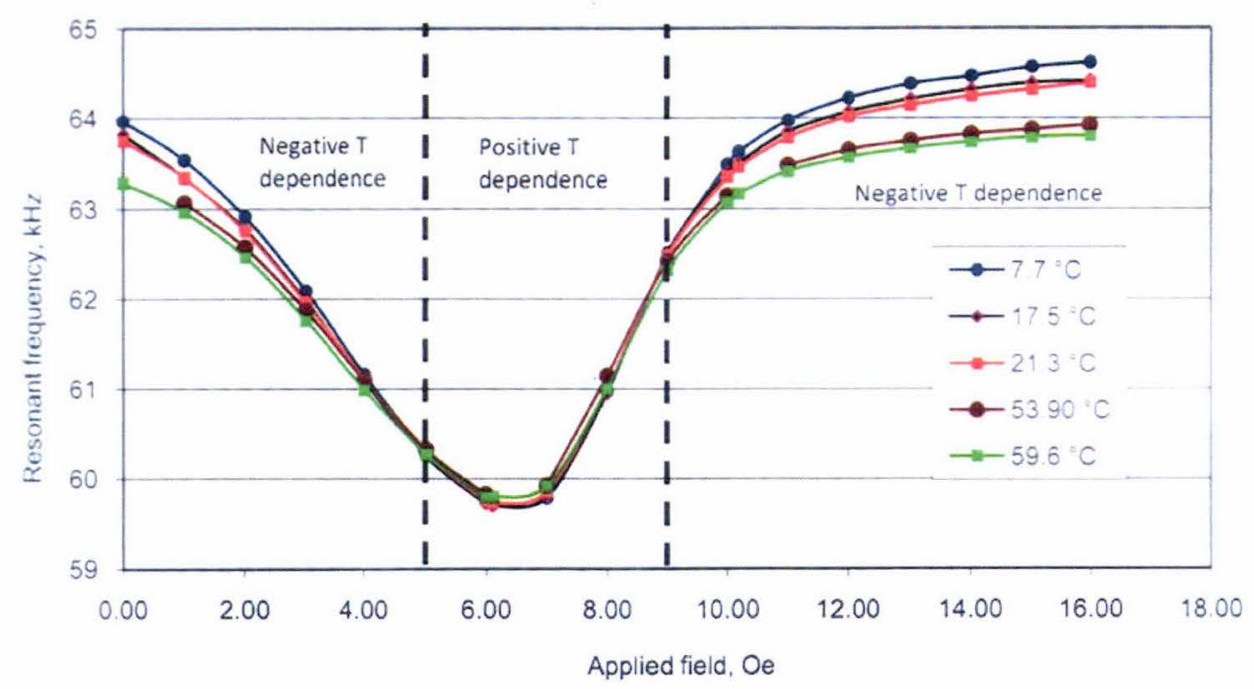

Figure 2.19: Previous results with temperature dependence [5]. 


\subsubsection{Detection Methods}

A number of methods are available to measure the resonant frequency of the magnetoelastic sensor; these include magnetic, optical, acoustic and impedance detuning methods [26]. The methods are summarised schematically in Fig. 2.20.

All methods (except impedance de-tuning) involve the excitation of the sensor with an AC magnetic field generated by an excitation (or driving) coil, and after this excitation is stopped, the sensor response is measured using the various methods. These different methods will now be briefly discussed. An extensive review of the application of magnetoelastic materials as sensors was done by Grimes et al [26].

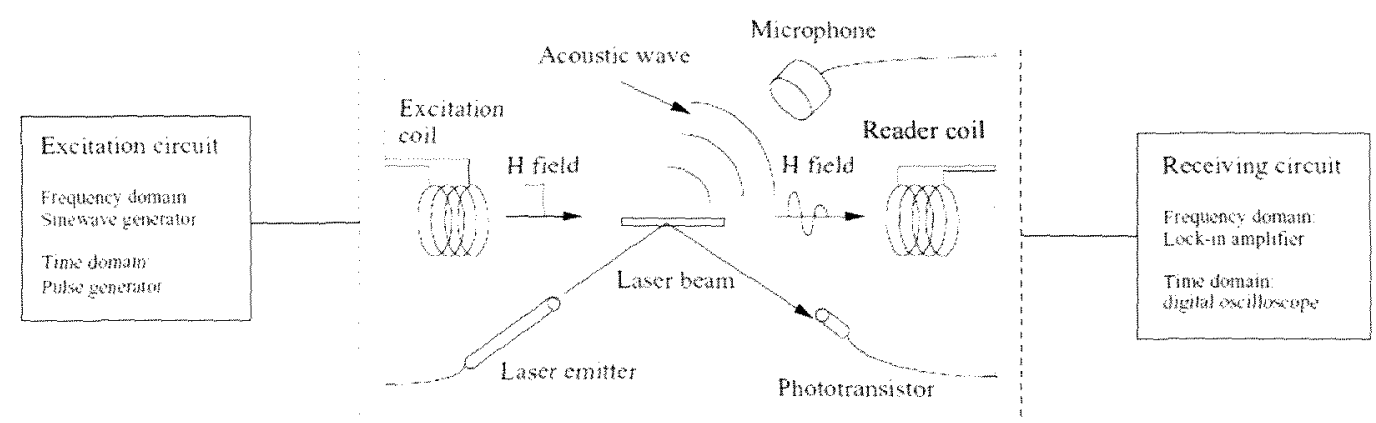

Figure 2.20: Methods to measure magnetoelastic sensors.

\subsubsection{Magnetic Method}

This method is similar to EAS systems found in stores. Once the sensor is excited by an AC magnetic field generated by the excitation coil, it will vibrate longitudinally, inducing small voltages in the reader coil, and will continue to vibrate after the excitation signal is stopped. This signal from the sensor is a decaying "ring-down" of the form shown previously in Fig. 2.3, and the amplitude of this signal is largest (and ring-down time longest) if the sensor was excited at its resonant frequency [26].

This signal is then analysed by techniques such as the Fast Fourier Transform (FFT) technique, looking for the frequency with the largest amplitude or by counting the number of oscillations for a given amount of time (frequency counting). It has been 
shown that the detection distance with this method is up to $30 \mathrm{~cm}$ for sensor $10 \mathrm{~cm}$ long.

\subsubsection{Acoustic Method}

Some groups have measured magnetoelastic sensors with the use of microphones [26]. This is done by placing a microphone near the sensor, and because the sensor produces acoustic waves as well as magnetic flux when vibrating, this can be picked up by the microphone which converts the sound waves into a voltage signal.

The advantage to this method is its detection range; it is longer than with the magnetic method described above, at up to $2 \mathrm{~m}$ for $10 \mathrm{~cm}$ sensors but with lower accuracy. A disadvantage to this method is that the sound waves can reflect if the sensor is placed inside a container.

\subsubsection{Optical Method}

Another method that is found in literature is by the use of a laser beam, which is reflected on the surface of the vibrating sensor [26]. The change in intensity of the reflected beam can be converted to an electrical signal with a phototransistor, and this signal is used to measure the sensor response.

The advantage to this method is long detection range; it is claimed that the maximum detection distance is up to $6 \mathrm{~m}$ for $1 \mathrm{~cm}$ long sensor. A disadvantage to this method would be the fact it requires a more complex detection system, reduced accuracy, as well as the need to have a clear line of sight with the laser. Such disadvantages outweigh the advantages and thus this method is rarely used.

\subsubsection{Impedance De-tuning Method}

An impedance analyser can be used to measure the resonant frequency of magnetoelastic materials by placing it inside a reader coil, which is connected to the terminals of the analyser [26]. The magnetoelastic material increases its magnetic permeability substantially at its resonant frequency and thus the inductance of the coil 
sharply increases at this point. This change in inductance is shown as a peak on the impedance plot against frequency.

Fig. 2.21 shows an example of a typical impedance and phase curve of a reader coil with a $40 \mathrm{~mm}$ long Metglas 2826MB strip inside, taken with an Agilent HP4294A impedance analyser. The impedance plot shows a sharp peak at resonance at about $55 \mathrm{kHz}$, a phase minimum at $55.2 \mathrm{kHz}$ and anti-resonance at $55.4 \mathrm{kHz}$ where the impedance is minimal.

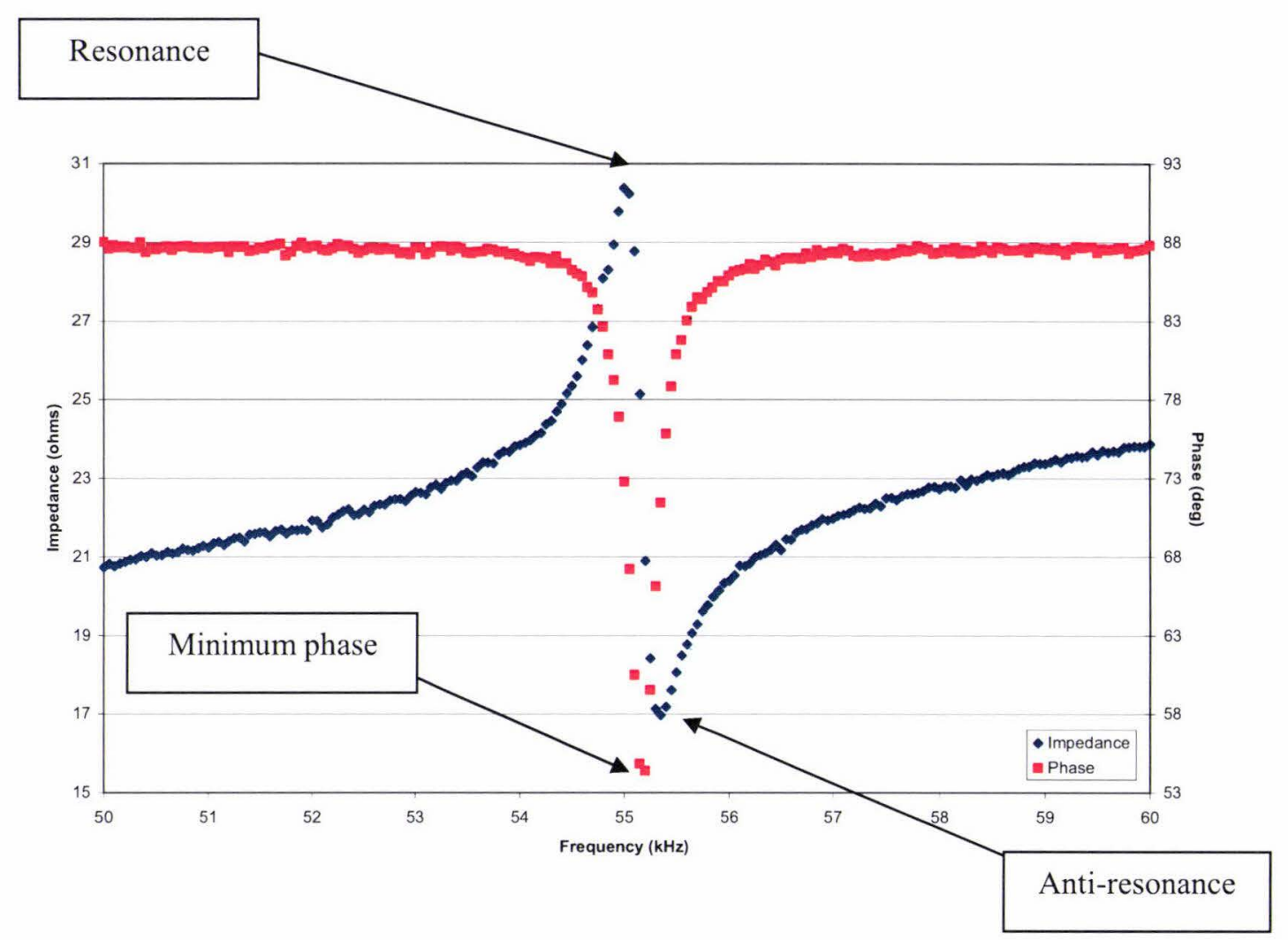

Figure 2.21: Impedance and phase plot of a reader coil with a Metglas 2826MB strip inside. The resonance, anti-resonance and minimum phase points are indicated.

\subsubsection{Literature Survey of Magnetoelastic Sensor Applications}

Magnetoelastic materials have been used to detect a large number of variables, including pressure, humidity, temperature, liquid viscosity/density, bacteria, thin-film elasticity, as well as chemicals such as carbon dioxide, ammonia and $\mathrm{pH}$ [26]. 
Below are brief descriptions to some recent papers which describe the use of magnetoelastic sensors.

Guntupalli et al [30] constructed a sensor for detecting the bacteria Salmonella typhimurium based on Metglas 2826MB ribbons. The Metglas strip was coated with an antibody to S. typhimurium and then exposed to different levels of the bacteria. The resonant frequency of the sensor was measured using an HP8751A network analyser set to do an S-parameter test. The paper reports a resonant frequency shift of $691 \mathrm{~Hz}$ for a concentration of $1 \times 10^{9} \mathrm{CFU} / \mathrm{mL}$ for $2 \times 0.4 \mathrm{~mm}$ sensors. Three different sizes of sensors were tested, and it was shown that larger ones have better detection rates.

Ong et al [31] describes the use of magnetoelastic sensors for detection of endotoxins produced by bacteria. The sensor was measured with an electronic box which has a drive (excitation) coil and a separate reader (receive) coil (see Fig. 2.22), with a microcontroller which controls operations. The block diagram of the system is also shown in Fig. 2.22. The system works by measuring the change in impedance of the reader coil due to the sensor by measuring the coil voltage and current. DC biasing is also built-in, and so the sensor does not require a biasing magnet. The claimed sensitivity of the system is $0.0105 \mathrm{EU} / \mathrm{ml}$.
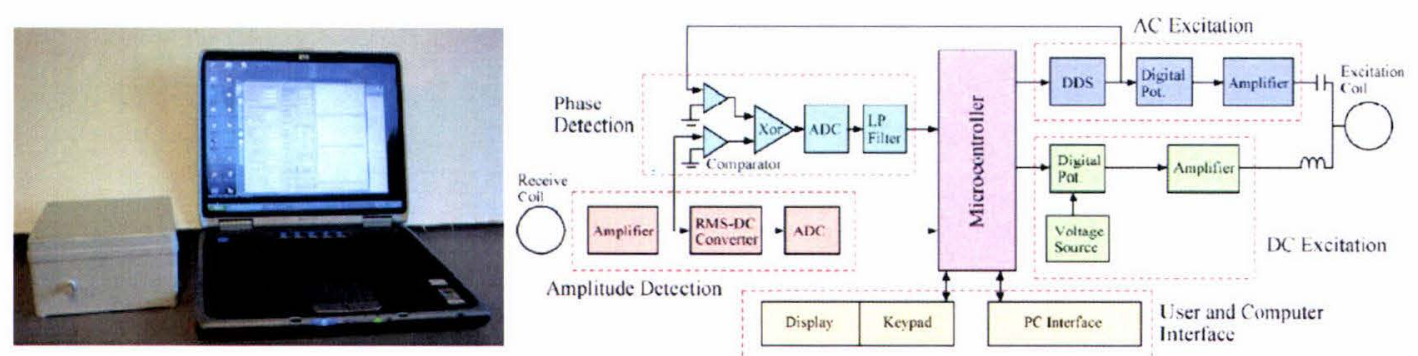

Figure 2.22: Detection system designed by Ong et al [31].

Pang el al [32] investigated magnetoelastic sensors for measurement of $\mathrm{pH}$ of human body fluid. The magnetoelastic material was Metglas $2826 \mathrm{MB}$, cut to resonate at 170 $\mathrm{kHz}$ in air, and coated with a $\mathrm{pH}$ sensitive polymer. The measurements were done using a system where the sensor is placed in a small vial which is surrounded by a coil, which is used both for receiving and excitation, as shown in Fig. 2.23. 
The coil is then connected to a box housing the electronics. It is stated that the frequency to $\mathrm{pH}$ relationship, in the linear range, was $0.2 \mathrm{kHz} / \mathrm{pH}$.

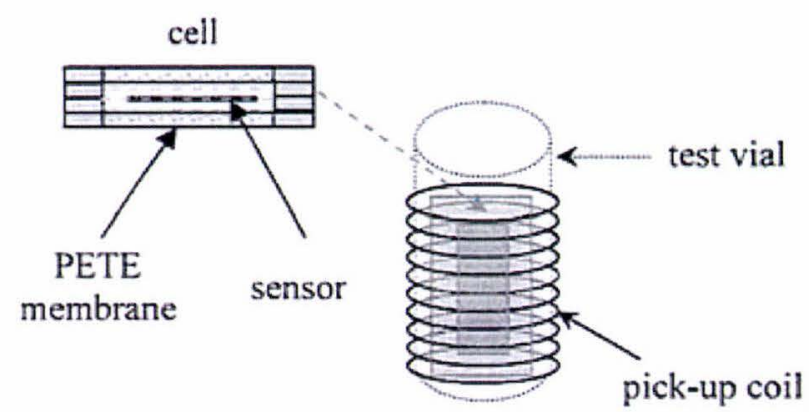

Figure 2.23: Measurement of $\mathrm{pH}$ with magnetoelastic sensors [32].

Wan et al [33] describes the use of magnetoelastic sensors for detection of the bacterial spores of Bacillus anthracis which can be used as biological weapons. The measurement system is shown in Fig. 2.24. The system uses two coils, one for DC biasing, and another for exciting and reading the sensor.

The sensor and reader coil arrangement is connected to a network analyser set to reflected impedance mode and the coil is swept in frequency from low to high. The paper claims that a frequency shift of $130 \mathrm{~Hz}$ was detected for an order of magnitude of spore concentration, while the detection limit was $10^{3}$ spores $/ \mathrm{ml}$.

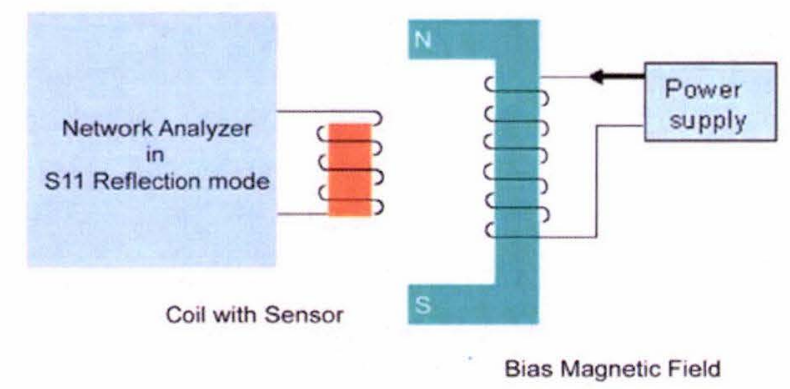

Figure 2.24: Detection of bacterial spores with magnetoelastic sensors [33] 


\section{Design and Construction of a Handheld Magnetoelastic Sensor Reader}

\subsection{Introduction}

From the initial work on magnetoelastic sensor work performed at SCPS by Smith [5], it became clear that the use of an impedance analyser and Helmholtz coil system for sensor readout is not practical for real-world applications. An impedance analyser is both expensive (cost approx. \$50,000NZ) and non-portable (weight approx. $40 \mathrm{~kg}$ ), making it impractical for applications outside the research laboratory.

One of the main goals of this project was then the design and construction of a prototype portable sensor reader. Such a reader will be of great benefit in using these sensors in real applications. In order to be practical, the reader will have to be small and light enough to be easily handled by an operator and provide a relatively simple user interface. At the same time it should also be sensitive enough to detect changes in the sensor's resonant frequency at a realistic distance.

A general system overview of the reader will be given in Section 3.2, with the various sub-systems discussed in more detail in Sections 3.3 to 3.10 . The printed circuit board (PCB) design and final system construction is described in Sections 3.11 and 3.12 with the reader performance evaluated in Section 3.13. The chapter is concluded with Section 3.14 which contains suggested improvements for a next generation reader. The entire schematic diagram can be found in Appendix A, PCB layout on Appendix B, while microcontroller source code in $\mathrm{C}$ can be found in Appendix C.

\subsection{System Overview}

This electronic reader is powered from a single $9 \mathrm{~V}$ battery or $\mathrm{AC}$ adapter and through a drive coil and a reader coil placed close to the sensor its resonant frequency can be measured and displayed on the LCD. The circuit was initially designed and prototyped on a breadboard, and then transferred onto a PCB. 
The block diagram of the system can be seen in Fig. 3.1.

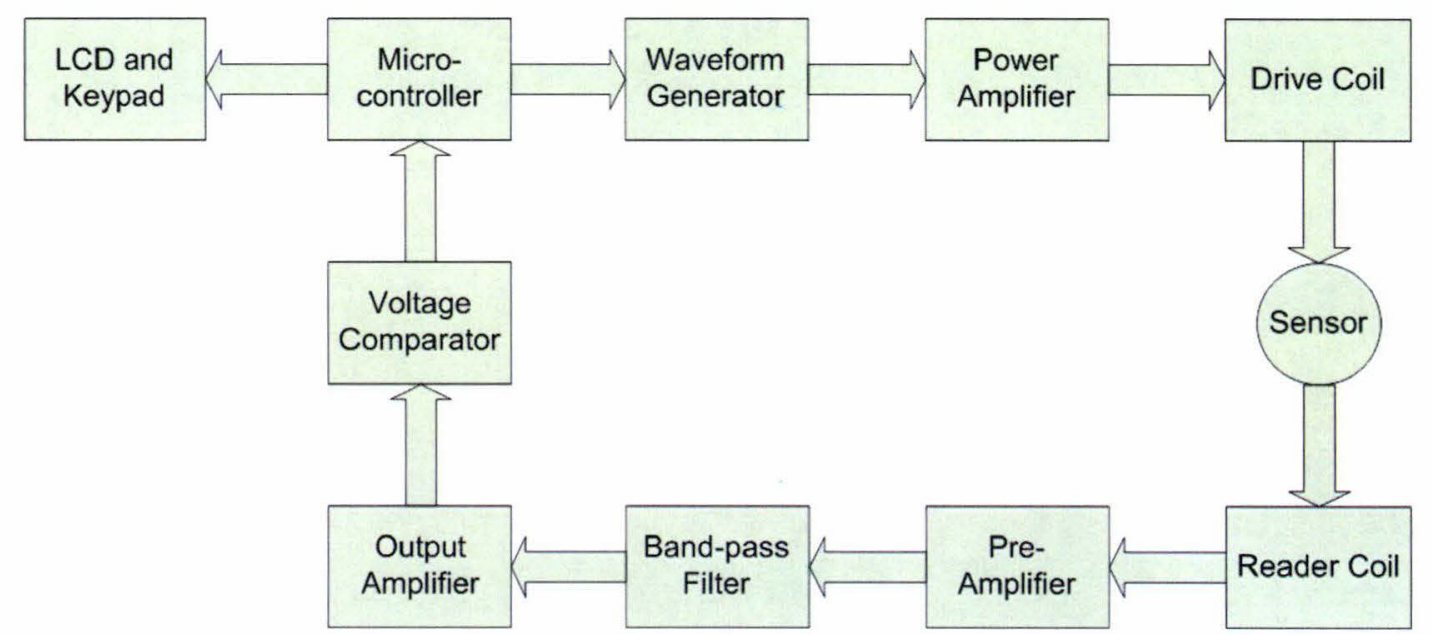

Figure 3.1: Block diagram of sensor reader.

The device is controlled by a central microcontroller, the Atmel AT89C51AC3. The microcontroller has a connected LCD and keypad which interfaces with the user. When the user asks for a measurement to start, the microcontroller through its Serial Peripheral Interface (SPI) port instructs the AD5930 waveform generator chip to produce an 80-cycle sine wave burst starting from a frequency lower than the expected resonant frequency, and the frequency is then gradually increased to above the expected resonant frequency. The signal from the waveform generator is fed into the SSM2211 power amplifier which is used to power the drive (excitation) coil, and this excites the sensor, causing it to vibrate.

The sensor, after being excited by the drive coil, vibrates through magnetostriction inducing a small voltage in the reader coil, and continues to vibrate after the driving signal is stopped, as illustrated in Fig. 3.2.

The signal from the reader coil is then fed into a series of operational amplifier circuits for conditioning. Firstly, it is amplified by a pre-amplifier, and then fed into an active band-pass filter. The filter is needed to remove high frequency noise as well as low frequency ringing present in the signal. The reason behind this combination is that because the sensor signal is very small, the filter does not work efficiently without being amplified first and can let through a lot of noise. The resulting signal is 
then amplified again with another amplifier with adjustable gain to give the user control over the amplitude of signal (and noise) being fed into the following stages.

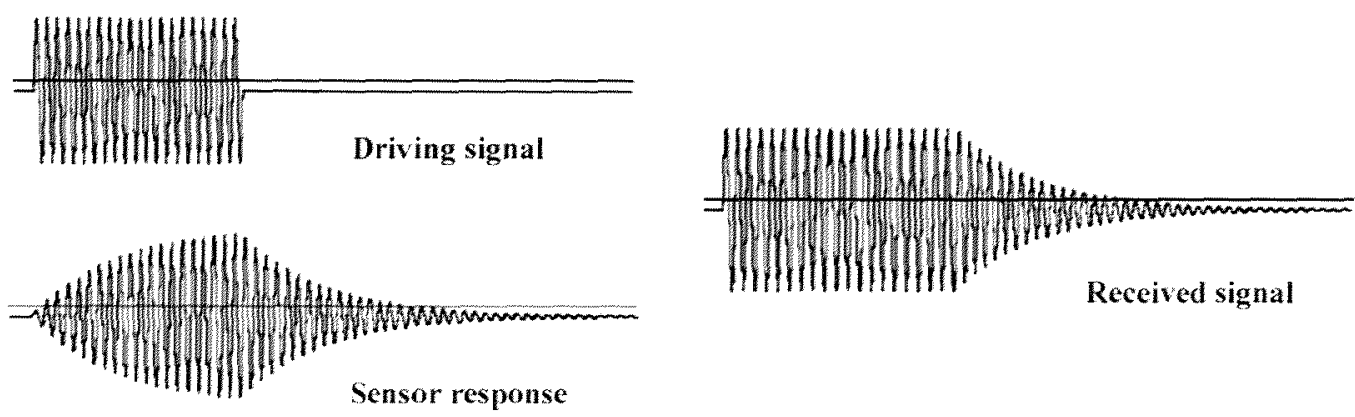

Figure 3.2: Driving signal (top left), sensor response (bottom left) and the actual received signal which is the sum of both parts.

The output from the amplifier is fed into an LM311 voltage comparator which converts the received signal to a series of digital pulses so it can be read by the microcontroller's internal counter. First, the driving signal is counted, and checked to see if it is the right length ( 80 pulses). After this is confirmed, the sensor response is counted. Since the ring-down of the sensor is longest if excited at its resonant frequency, the frequency at which the output of this comparator is longest is identified as the sensor's resonant frequency by the microcontroller. This effect is illustrated in the plot of Fig. 3.3, which shows the ring-down length of a magnetoelastic sensor, designed to resonate at about $55.5 \mathrm{kHz}$. The sensor is driven at different frequencies and it is clear from the plot that the sensor strip responds most strongly if driven near its resonant frequency.

The reference voltage for the comparator is set manually with a trimming potentiometer, which is connected as a voltage divider from $+5 \mathrm{~V}$ to ground. This voltage is normally set to about $2 \mathrm{~V}$, which is just above the noise level measured in the system. The comparator converts the signal to a series of digital pulses so that the microcontroller can use its internal counters to find the number of pulses received from the sensor for each frequency. The frequency which gives the largest number of pulses (thus the longest oscillation) from the sensor is identified as the sensor's resonant frequency. At each frequency the reader waits for $5 \mathrm{~ms}$ to ensure that all the sensor pulses are counted. The time of $5 \mathrm{~ms}$ is used as this was found to be long 
enough to account for the sensor's ring-down time even if placed very close to the reader coil.

The above method was found to be the most noise immune and CPU efficient method for measuring the resonant frequency of magnetoelastic sensors. Other methods such as feeding the pulses into the microcontroller's external interrupt or Program Counter Array (PCA) capture input, while measuring the time taken for a given number of pulses was also investigated, but this was inefficient use of the microcontroller's CPU resources as this requires interrupts to be serviced every few microseconds, as well as being very susceptible to noise effects. Alternatively, the onboard analogue to digital converter could have been used to analyse the sensor signal. This however was not considered due to the requirement of a more complex detection method required to analyse the incoming $\mathrm{AC}$ waveform.

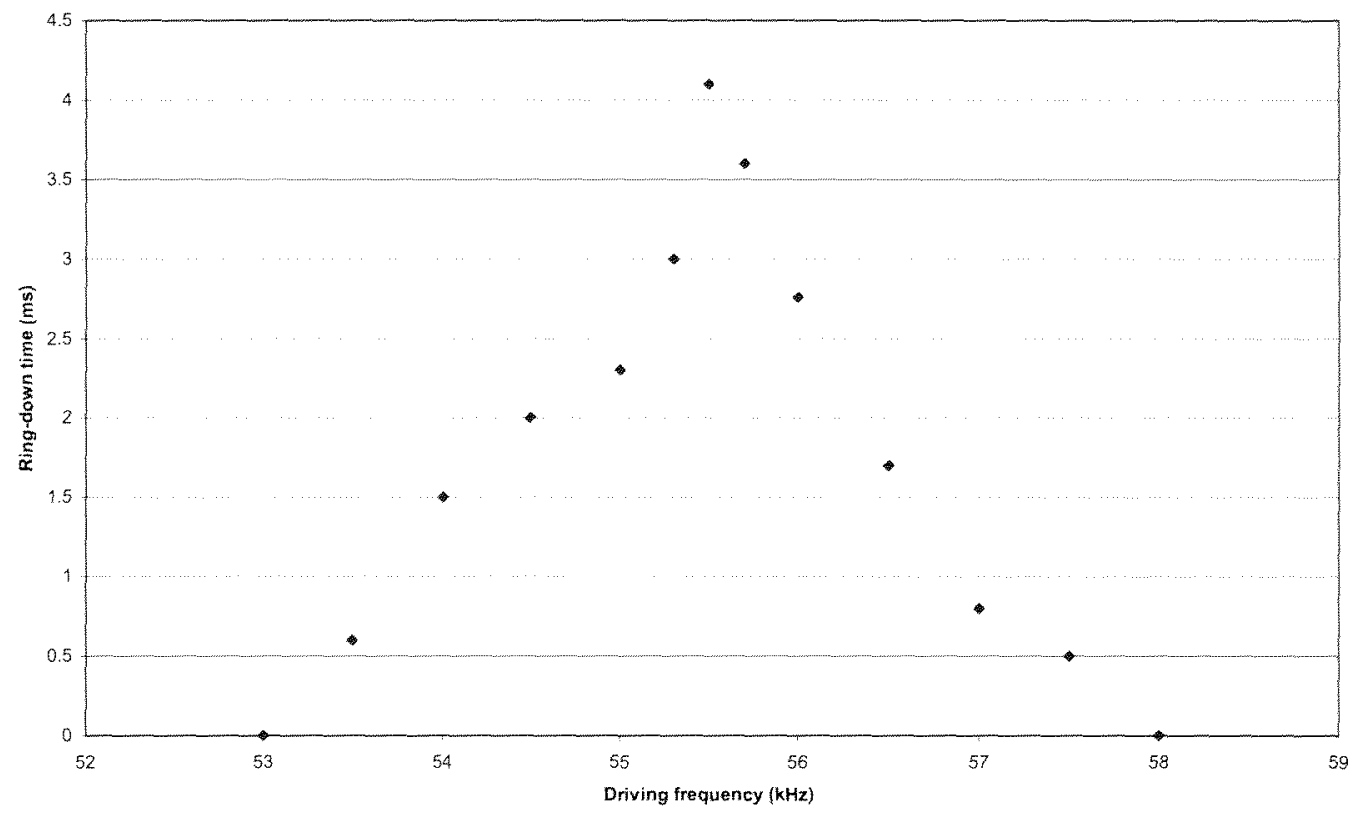

Figure 3.3: Driving frequency versus sensor ring-down time.

\subsection{Microcontroller}

The microcontroller used was an Atmel AT89C51AC3 [34] with the Plastic Leaded Chip Carrier 52-pin (PLCC52) package. This IC costs approximately \$18NZ. This was used due to its familiarity, and its specifications were found to be adequate for 
this purpose, and this package was used due to the convenience of being able to replace it easily with a socket. The AT89C51AC3 is an 80C51-based 8-bit microcontroller with the following internal features:

- 256 Bytes of RAM.

- 2048 Bytes of ERAM.

- 64 Kbytes of Flash memory.

- 2 Kbytes of EEPROM.

- In System Programming (ISP) capable.

- Five IO ports.

- Five channel Programmable Counter Array (PCA).

- Three 16-bit timer/counter modules.

- 10-bit Analogue to Digital Converter (ADC).

- Serial Peripheral Interface (SPI).

- Power saving function.

- Full duplex Universal Asynchronous Receiver Transmitter (UART).

The AT89C51AC3 is operated at $20 \mathrm{MHz}$ in X2 mode (a faster speed means measurements are completed in less time), and its firmware code was written in ANSI-C, using the programming environment HI-TIDE from HI-TECH software [35]. The compiled code is sent to the microcontroller through either a USB port (using an external serial-USB adapter) or serial (RS-232) port on a computer using Atmel's FLIP (Flexible In-system Programmer) software [36].

The IO ports of the microcontroller are utilised as follows:

- Port 0 :

- Data bits to LCD.

- Port 1:
- Pin 0: STANDBY signal to AD5930.
- Pin 1: Timer2 up/down control.
- Pin 2: SHUTDOWN signal to SSM2211.
- Pin 3: ENABLE signal to $50 \mathrm{MHz}$ oscillator.
- Pin 4: SYNCOUT signal from AD5930.
- Pin 5: MSBOUT signal from AD5930. 
- Pin 6: INTERRPUT signal to AD5930.

- Pin 7: Not used.

- Port 2:

- Keypad input.

- Port 3:

- Pin 0: UART RxD.

- Pin 1: UART TxD.

- Pin 2: RS signal to LCD.

- Pin 3: RW signal to LCD.

- Pin 4: Timer0 counter input.

- Pin 5: Not used.

- Pin 6: EN signal to LCD.

- Pin 7: Not used.

- Port 4:

- Pin 0: CONTROL signal to AD5930.

- Pin 1: FSYNC signal to AD5930.

- Pin 2: Not used.

- Pin 3: SCLK signal to AD5930.

- Pin 4: SDATA signal to AD5930.

The block diagram of the overall program flow of the microcontroller code is shown in Fig. 3.4. The program is divided into four $\mathrm{C}$ source files and their corresponding header files; they are listed below:

- main.c:

The main program code, which contains the main() function.

- keypad.c:

Contains functions to read the keypad.

- 1cd.c:

Contains functions to control the LCD, as well as simple string manipulation.

- Setupfunctions.c:

Contains functions to initialise registers for the timers, SPI etc. 
The output of the comparator is connected to Timer0, one of the microcontroller's onboard timer/counter modules, which is operated as an externally triggered 16-bit counter. For each frequency, the initial 80-cycle excitation signal is detected by this counter. While this is being done, Timer2 is operated as a 16-bit auto-reload timer, which checks how long it is taking to finish detection of the drive signal. If the time is longer than expected, the microcontroller identifies an error in the drive signal, and stops the measurement. This is required as otherwise if there is no detected drive signal, the microcontroller enters an infinite loop.

Once the drive signal is detected, the number of pulses produced by the sensor is counted. Because the number of sensor pulses expected is not known before the measurement (i.e. we do not know when the last sensor pulse will arrive), Timer2 is reset from the previous section and again operated as a 16-bit auto-reload timer, and run in order to set a time limit on when to stop sampling at. This time limit is set to about $5 \mathrm{~ms}$ which is close to the ring-down time when the sensor is very close to the reader coil.

At each frequency, the number of pulses arriving after the initial 80-cycle excitation burst (i.e. the sensor pulses) is counted and compared to the previous maximum. If the current count is larger than the previous maximum count, the current count is set as the maximum, and the process is repeated for each frequency. The frequency is incremented by toggling pin 0 of port 4 on the microcontroller, connected to the CONTROL line on the waveform generator. The current frequency is known from the number of times the CONTROL line was toggled.

This method works because, as shown in Fig. 3.3 the length of ringing produced by the sensor is in the form of a triangle; the number of pulses starts off small, increases gradually until a maximum value at the resonant frequency, and then gradually deceases, so the maximum value is always saved at the end. Finally, the maximum sensor pulses obtained is checked to see if it is greater than 40 . This ensures that the "sensor" pulses were not caused simply by noise. The value of 40 was chosen as it was found that when the system was run without a sensor present, it would detect about 30 pulses, caused by noise effects. 
The frequency which gave the largest number of pulses is then identified as the resonant frequency of the sensor, and this is then converted to the environmental parameter being measured with a floating point calculation.

One point to note, which is not shown in Fig. 3.4, is that in the sensor measurement loop there also is some checking code run every 50 iterations which polls the keypad for an input on key 2. If the key is pressed, the measurement is cancelled. The reason for doing this every 50 cycles is because this is slow, and can hold up the measurement. Doing this every 50 iterations was found to be sufficient, requiring the key to be pressed for about 2 seconds.

The entire measurement process takes about 1.5 minutes to analyse 2000 frequencies. Since the time taken for the measurement is mainly dependant on the waiting time at each frequency $(5 \mathrm{~ms})$, the use of a faster microcontroller would not result in a significantly shorter measurement time.

\subsection{LCD and Keypad}

The LCD and keypad are used to interface with the user, and are directly connected to the microcontroller. The connections of these parts are discussed in this section.

\subsubsection{LCD}

The LCD module is a Seiko Instruments L1672 which is an alphanumeric 16 character $\times 2$ line dot matrix display, which costs approximately $\$ 11 \mathrm{NZ}$. Table 3.1 summarises the pin functions on the LCD as well as how they are connected. The data bits are connected to IO port 0 of the microcontroller, while the control lines are connected to port 3. DGND means digital ground, while DVCC refers to the digital $+5 \mathrm{~V}$ power line. The driving voltage $\mathrm{V}_{\mathrm{LC}}$ (pin 12) can be used to adjust the contrast of the LCD, however this was done in this application. 


\subsubsection{Keypad}

The keypad is connected to IO port 2 on the microcontroller. During prototyping, an ECO series numeric 12-way keypad was used but in the final design this was replaced with two pushbuttons in order to save space on the case. Also, the fact that other than keys 1 and 2, no other keys were being used meant having a keypad with 12 keys was unnecessary.

The buttons are connected as shown in Fig. 3.5. The buttons are activated by setting IO port 2 pin 0 to LOW output, and connecting the buttons to short the connection between this pin to input pins 4 (key/button 1) and 5 (key/button 2) on port 2. A LOW read from these lines indicates that the button is activated.

The buttons used were MCS1 8 series panel-mount pushbuttons from Schurter, which are "snapped" into place to holes on the case. The buttons cost approximately $\$ 6.80 \mathrm{NZ}$ each. 


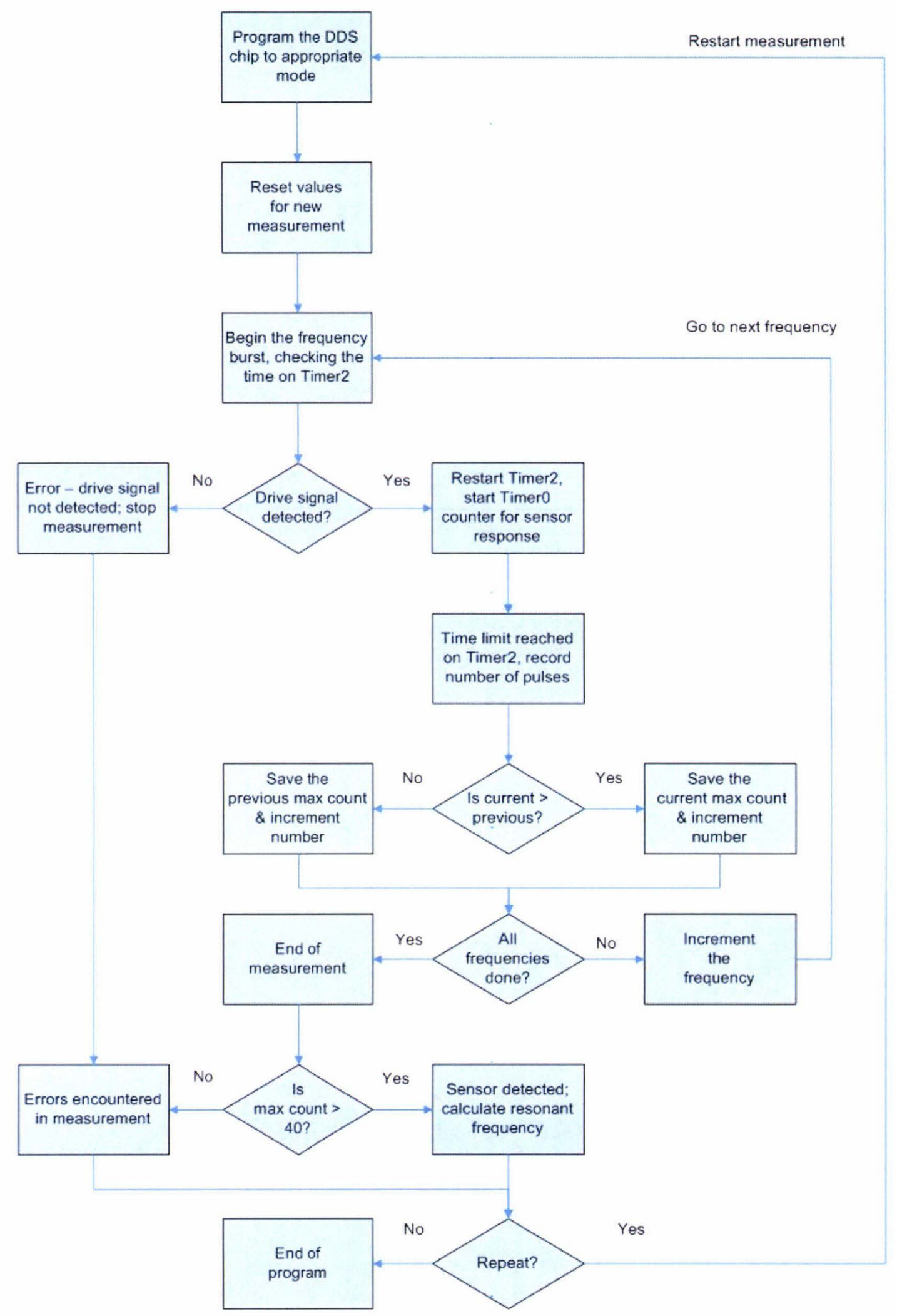

Figure 3.4: Program flow of microcontroller. 
Table 3.1: Pin functions and connection for the LCD module.

\begin{tabular}{|c|c|c|c|}
\hline Pin no. & Name & Function & Connection \\
\hline $\mathbf{1}$ & DB7 & Data bit & P0.7 \\
\hline $\mathbf{2}$ & DB6 & Data bit & P0.6 \\
\hline $\mathbf{3}$ & DB5 & Data bit & P0.5 \\
\hline $\mathbf{4}$ & DB4 & Data bit & P0.4 \\
\hline $\mathbf{5}$ & DB3 & Data bit & P0.3 \\
\hline $\mathbf{6}$ & DB2 & Data bit & P0.2 \\
\hline $\mathbf{7}$ & DB1 & Data bit & P0.1 \\
\hline $\mathbf{8}$ & DB0 & Data bit & P0.0 \\
\hline $\mathbf{9}$ & EN & Enable signal & P3.6 \\
\hline $\mathbf{1 0}$ & RW & Read/Write signal & P3.2 \\
\hline $\mathbf{1 1}$ & RS & RS signal & DGND \\
\hline $\mathbf{1 2}$ & V & Driving voltage & DGND \\
\hline $\mathbf{1 3}$ & V & GND & DVCC \\
\hline $\mathbf{1 4}$ & V & DD & +5 V power supply \\
\hline
\end{tabular}

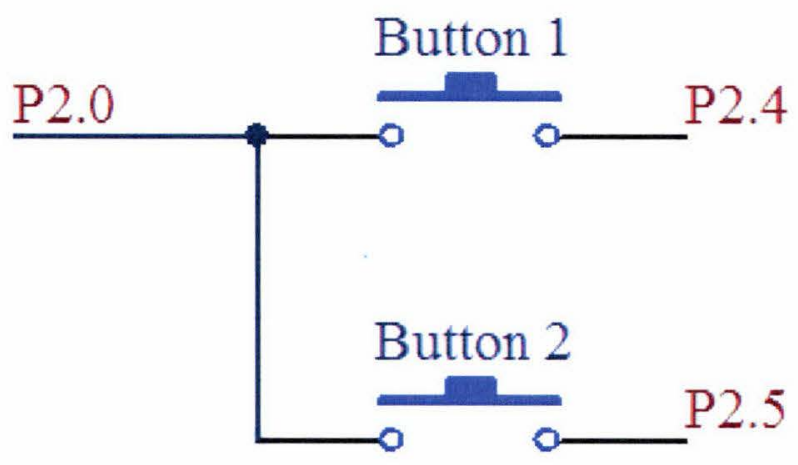

Figure 3.5: Connection for the pushbuttons.

\subsection{Waveform Generator}

The waveform generator, used to produce the excitation signals, was chosen as a chip from Analog Devices' family of Direct Digital Synthesisers (DDS). Direct Digital Synthesis is a technique to produce accurate sine, triangular and square waves from an accurate clock source. 
Three DDS chips were found from New Zealand distributors Farnell and RS:

- AD9852 Complete DDS

- AD5930 Waveform Generator

- AD5932 Waveform Generator

The AD9852 [37] is a complete DDS, which means it has many features which would not be used for such an application. The chip was also expensive (approx. \$140NZ), and requires significant amount of power (in fact it requires a thermal pad on the PCB). The AD5930 [38] and AD5932 [39] chips are much simpler, lower-cost alternatives which would be more suited for such an application. Out of the two, the AD5930 was chosen as it has a Burst Mode - the ability to produce a waveform for a set period then idle, a function which was not available on the AD5932. The AD5930 was slightly more expensive (approx. \$37NZ) than the AD5932 (approx. \$30NZ) due to this added feature. The AD5930 is programmed through a 3-wire serial interface with a microcontroller or microprocessor, such as Serial Peripheral Interface (SPI), Queued Serial Peripheral Interface (QSPI) or Microwire.

The AD5930 is labelled by Analog Devices as a "Programmable Frequency Scan and Output Burst Waveform Generator", intended for low power applications. The device comes in a surface mounted 20-pin Thin Shrink Small Outline Package (TSSOP), taking little space on the PCB. The device can produce continuous or burst (produce for a set length of time) waveforms with increasing frequency in the following way:

$$
f=F_{\text {start }}+(\Delta F \times n)
$$

Where $f$ is the current frequency, $F_{\text {start }}$ is the initial frequency, $\Delta F$ is the increment size and $n$ is the $n^{\text {th }}$ frequency increment. The three parameters are pre-programmed and thus remove the need for continuous data transfers from the microcontroller.

Since this IC is only available in surface mount packages, during prototyping, a simple PCB was constructed to accommodate the AD5930, with connected leads to interface with the breadboard. 


\subsubsection{Circuit Setup}

The circuit for the AD5930 was designed to closely resemble Analog Devices' evaluation board [40]. The schematic for the AD5930 with supporting components is shown in Fig. 3.6. The device is powered from two $5 \mathrm{~V}$ power lines AVCC and DVCC, which are separated with a bead inductor to reduce noise.

The clock source (MCLK) used was a $5 \mathrm{~V}$ powered SG-8002JA series [41] surface mount oscillator from Epson (cost approx. $\$ 10.60 \mathrm{NZ}$ ) producing a sine wave of $50 \mathrm{MHz}$ between $5 \mathrm{~V}$ and $0 \mathrm{~V}$. The choice was due to Analog Devices recommending the Epson brand on their website, claimed as being accurate and reliable [42]. Because the output frequency is derived from this clock source, the maximum and minimum output frequencies are limited by this oscillator frequency. For this case it is $3 \mathrm{~Hz}$ up to $25 \mathrm{MHz}$. If further resolution is required, the oscillator can be replaced with a lower speed version in the same series, with a few modifications to the microcontroller code.

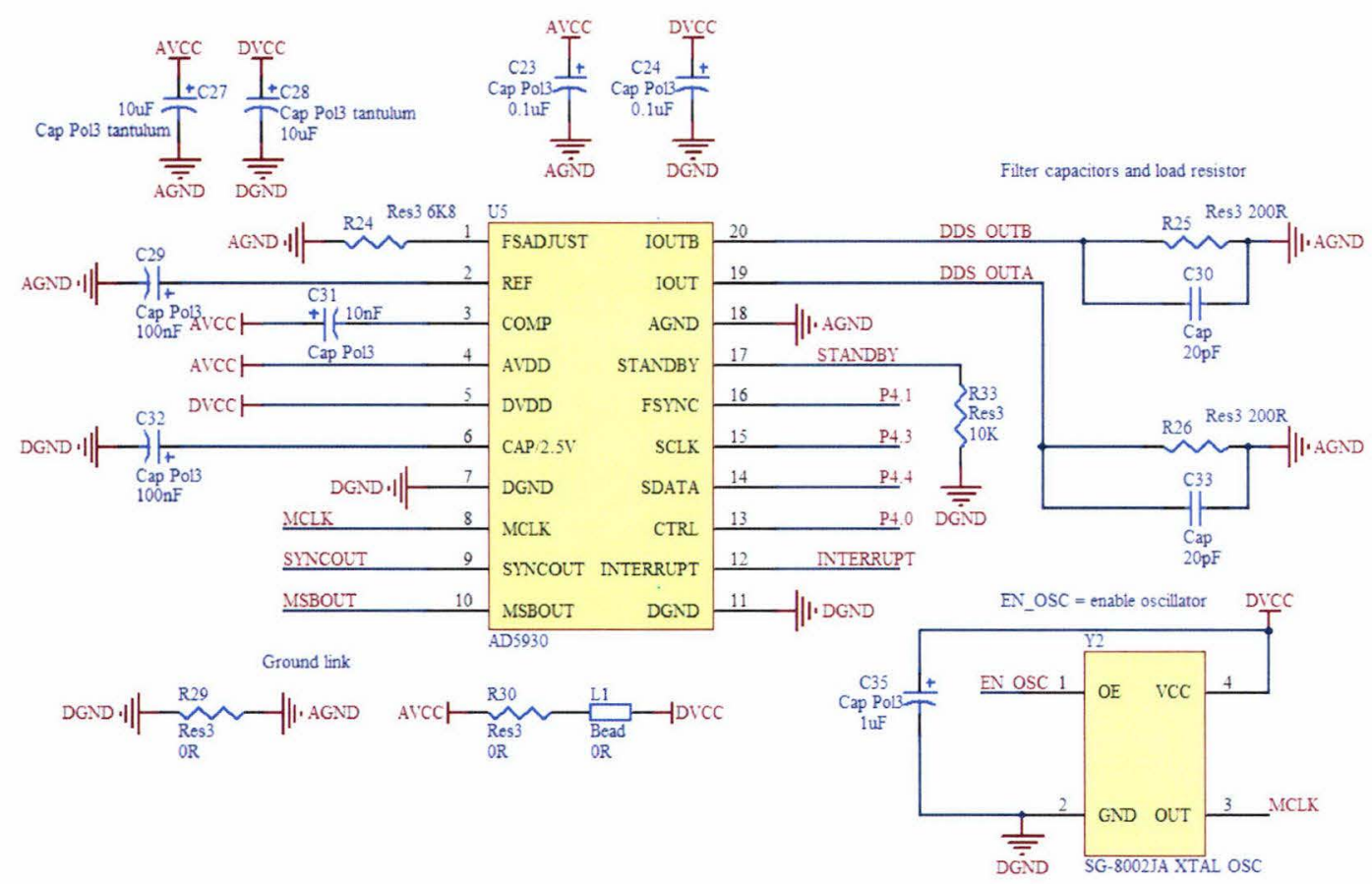

Figure 3.6: Schematic of waveform generator circuit with supporting components. 
At the outputs (IOUT and its complement, IOUTB) of the AD5930, there is a $200 \Omega$ resistor and a $20 \mathrm{pF}$ capacitor to ground. The resistor acts as a load in order to convert the current output to a voltage and the capacitor in parallel to it acts as a low pass filter to reduce clock signal feed through. The resistor at FSADJUST pin sets the fullscale output current. The resistance-to-current relationship is:

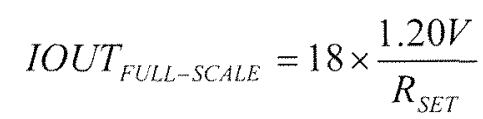

Where the $1.20 \mathrm{~V}$ is the reference voltage found at pin 2 . Nominally $R_{S E T}$ is set to $6.8 \mathrm{k} \Omega$, giving full-scale current of approximately $3.2 \mathrm{~mA}$, thus with a $200 \Omega$ load the output will oscillate approximately between 0 and $0.7 \mathrm{~V}$ (measured to be closer to $0.8 \mathrm{~V})$

One may notice the pins labelled SYNCOUT and MSBOUT. The two pins are connected to the microcontroller (SYNCOUT to port 1 pin 4 and MSBOUT to port 1 pin 5), but are currently not used. The former is intended to signal the end of a frequency sweep, and the latter is intended to produce a square wave output. It was thought that the output from SYNCOUT could be used instead of counting the 80 -cycle excitation burst but this could not be achieved.

In order to save power, the STANDBY pin can be set HIGH, causing the AD5930 to idle. The STANDBY pin is connected to port 1 pin 0 on the microcontroller but this pin had to be disabled with the pull-down resistor R33 because the IO ports of the microcontroller normally idle $\mathrm{HIGH}$ and thus can activate the pin at start-up, causing the chip to enter STANDBY state when an output is needed. If the AD5930 requires idling, the ENABLE pin on the SG-8002JA oscillator can be set LOW. This pin is connected to port 1 pin 3 on the microcontroller and it causes the oscillator to stop producing its output, resulting in lower power consumption [42].

The INTERRUPT pin is connected to port 1 pin 6 on the microcontroller and by applying a LOW-to-HIGH transition to this pin, the AD5930 can be put into INTERRUPT state, temporarily halting its operation. Normally this line should be set to LOW. 


\subsubsection{Programming the Waveform Generator}

The AD5930 waveform generator is programmed through a serial data connection using the microcontroller's SPI, found on IO port 4. The microcontroller is programmed as Master and its Slave Select (SS) pin is disabled as there are no other SPI devices in the system.

The AD5930 accepts 16-bit instructions while the microcontroller is 8-bit. To work around, an instruction is transferred in two separate bytes. The serial clock idles HIGH between the two bytes, and one of the IO lines (FSYNC) is held low while the two bytes are being sent to indicate a single instruction. These settings for the SPI are defined by the register SPCON on the microcontroller. The 16-bit instruction consists of 4 register address bits and 12 data bits, with the address bits being sent first. The device requires four pins for programming and control:

- CONTROL (CTRL)

This pin is used to determine when the device is in programming mode (it is logic LOW when it is) or not, as well as being the external control for starting and changing the output by toggling from LOW to HIGH. Output frequencies are also incremented by toggling this line. The microcontroller's port 4 , pin 0 is used for this line.

- FRAME SYNCHRONIZATION (FSYNC)

This is equivalent to a Chip Select (CS) on other SPI devices. It is held LOW while two bytes of the same instruction are being transferred to the device. The microcontroller's port 4 , pin 1 is used for this line.

- SERIAL CLOCK (SCK)

This is the serial clock signal of the SPI. It is found on port 4, pin 3 on the microcontroller.

- SERIAL DATA (SDATA)

This is the serial data signal. It is labelled as Master Out Serial In (MOSI) on the microcontroller, at port 4 , pin 4 . 
A number of registers in the AD5930 must be programmed before use. They are shown in the following sections.

\subsubsection{Control Register}

The Control Register has the address $0 \times 0$ and sets up the operating modes of the AD5930. It is programmed to run in External Increment, Auto-Burst Mode. This implies that the frequency increment is controlled by LOW-to-HIGH transitions on the CTRL line, and the length of the burst is pre-programmed. The output idles at midscale after the burst until the CTRL line is toggled again. The transferred data are shown in Table 3.2. The top row is the bit number, and bottom shows the data. The shaded region indicates the address bits.

Table 3.2: Transferred data for the Control Register.

\begin{tabular}{|l|l|l|l|l|l|l|l|l|l|l|l|l|l|l|l|l|}
\hline $\mathbf{1 5}$ & $\mathbf{1 4}$ & $\mathbf{1 3}$ & $\mathbf{1 2}$ & $\mathbf{1 1}$ & $\mathbf{1 0}$ & $\mathbf{9}$ & $\mathbf{8}$ & $\mathbf{7}$ & $\mathbf{6}$ & $\mathbf{5}$ & $\mathbf{4}$ & $\mathbf{3}$ & $\mathbf{2}$ & $\mathbf{1}$ & $\mathbf{0}$ & Value \\
\hline 0 & 0 & 0 & 0 & 1 & 1 & 1 & 0 & 0 & 0 & 1 & 1 & 0 & 0 & 1 & 1 & $0 x 0 \mathrm{E} 33$ \\
\hline
\end{tabular}

For External Increment, Auto-Burst Mode, the CW/BURST bit (bit 7) must be 0, INT/EXT BURST bit (bit 6) must be 0 and INT/EXT INCR (bit 5) must be 1 .

The other bits that must be set are bits 0 and 1 which must always be 1, SINE/TRI (bit 9) which must be 1 to give a sinusoidal output, DAC ENABLE (bit 10) which must be 1 to enable the output digital-to-analogue converter (DAC) and B24 (bit 11) which must be 1 to enable 2 write operations to load a complete word into the $\mathrm{F}_{\text {start }}$ and $\Delta \mathrm{F}$ registers. MODE (bit 4) is set to 1 which gives a saw-sweep frequency profile, implying that once the maximum frequency is reached, the sweep simply restarts from $\mathrm{F}_{\text {start }}$. Setting this bit to 0 gives a triangular frequency profile meaning that the once the maximum frequency is reached, the frequency will decrement from maximum down to $\mathrm{F}_{\text {start }}$ and then restart.

The remaining bits SYNCOUTEN (bit 2) and SYNCSEL (bit 3) control the output at the SYNCOUT pin, while the bit MSBOUTEN (bit 8) controls the MSBOUT pin. Since these functions are currently not used they are set to 0 . 


\subsubsection{2 $\mathrm{F}_{\text {start }}$ and $\Delta \mathrm{F}$ Registers}

The two $\mathrm{F}_{\text {start }}$ registers (addresses $0 \mathrm{xC}$ and $0 \mathrm{xD}$ ) contains the least significant bits (LSB) and most significant bits (MSB) respectively of the initial frequency to start from. The frequency value (the Frequency Tuning Word, FTW) is calculated as:

$$
F T W=\frac{f_{\text {wanted }}}{\left(f_{\text {osc }} / 2^{24}\right)}
$$

Where $f_{\text {wanted }}$ is the wanted frequency, $f_{\text {osc }}$ is the Master Clock frequency (MCLK) provided by the oscillator and 24 refer to the fact that the core is 24-bit. The start frequency is programmed to $52 \mathrm{kHz}$, thus the FTW is $0 \mathrm{x} 004428$. The least significant bits are programmed to address $0 \mathrm{xC}$ and the data sent are shown in Table 3.3. Note that this is split into 2 bytes during programming as it is 16-bit.

Table 3.3: Transferred data for the $\mathrm{F}_{\text {start, }}$ low bits.

\begin{tabular}{|l|l|l|l|l|l|l|l|l|l|l|l|l|l|l|l|l|}
\hline $\mathbf{1 5}$ & $\mathbf{1 4}$ & $\mathbf{1 3}$ & $\mathbf{1 2}$ & $\mathbf{1 1}$ & $\mathbf{1 0}$ & $\mathbf{9}$ & $\mathbf{8}$ & $\mathbf{7}$ & $\mathbf{6}$ & $\mathbf{5}$ & $\mathbf{4}$ & $\mathbf{3}$ & $\mathbf{2}$ & $\mathbf{1}$ & $\mathbf{0}$ & Value \\
\hline 1 & 1 & 0 & 0 & 0 & 1 & 0 & 0 & 0 & 0 & 1 & 0 & 1 & 0 & 0 & 0 & $0 \times \mathrm{x} 428$ \\
\hline
\end{tabular}

The most significant bits of the instruction are programmed to address $0 \mathrm{xD}$, the data are shown in Table 3.4.

Table 3.4: Transferred data for the $\mathrm{F}_{\text {start }}$, high bits.

\begin{tabular}{|l|l|l|l|l|l|l|l|l|l|l|l|l|l|l|l|l|}
\hline $\mathbf{1 5}$ & $\mathbf{1 4}$ & $\mathbf{1 3}$ & $\mathbf{1 2}$ & $\mathbf{1 1}$ & $\mathbf{1 0}$ & $\mathbf{9}$ & $\mathbf{8}$ & $\mathbf{7}$ & $\mathbf{6}$ & $\mathbf{5}$ & $\mathbf{4}$ & $\mathbf{3}$ & $\mathbf{2}$ & $\mathbf{1}$ & $\mathbf{0}$ & Value \\
\hline 1 & 1 & 0 & 1 & 0 & 0 & 0 & 0 & 0 & 0 & 0 & 0 & 0 & 1 & 0 & 0 & $0 x D 004$ \\
\hline
\end{tabular}

The delta frequency word $(\Delta \mathrm{F})$ registers determines the step size in frequency increment, and it is calculated and programmed in the same way. This is set to the smallest value of $0 \times 1$ giving $3 \mathrm{~Hz}$. The transferred data for the low order bits are transferred to address $0 \times 2$ and is shown in Table 3.5. 
Table 3.5: Transferred data for the $\Delta \mathrm{F}$, low bits.

\begin{tabular}{|l|l|l|l|l|l|l|l|l|l|l|l|l|l|l|l|l|}
\hline $\mathbf{1 5}$ & $\mathbf{1 4}$ & $\mathbf{1 3}$ & $\mathbf{1 2}$ & $\mathbf{1 1}$ & $\mathbf{1 0}$ & $\mathbf{9}$ & $\mathbf{8}$ & $\mathbf{7}$ & $\mathbf{6}$ & $\mathbf{5}$ & $\mathbf{4}$ & $\mathbf{3}$ & $\mathbf{2}$ & $\mathbf{1}$ & $\mathbf{0}$ & Value \\
\hline 0 & 0 & 1 & 0 & 0 & 0 & 0 & 0 & 0 & 0 & 0 & 0 & 0 & 0 & 0 & 1 & $0 \times 2001$ \\
\hline
\end{tabular}

Similarly to the above the high order bits are transferred to address $0 \times 3$ with the data shown in Table 3.6.

Table 3.6: Transferred data for the $\Delta \mathrm{F}$, high bits.

\begin{tabular}{|l|l|l|l|l|l|l|l|l|l|l|l|l|l|l|l|l|}
\hline $\mathbf{1 5}$ & $\mathbf{1 4}$ & $\mathbf{1 3}$ & $\mathbf{1 2}$ & $\mathbf{1 1}$ & $\mathbf{1 0}$ & $\mathbf{9}$ & $\mathbf{8}$ & $\mathbf{7}$ & $\mathbf{6}$ & $\mathbf{5}$ & $\mathbf{4}$ & $\mathbf{3}$ & $\mathbf{2}$ & $\mathbf{1}$ & $\mathbf{0}$ & Value \\
\hline 0 & 0 & 1 & 1 & 0 & 0 & 0 & 0 & 0 & 0 & 0 & 0 & 0 & 0 & 0 & 0 & $0 \times 3000$ \\
\hline
\end{tabular}

\subsubsection{Number of Increments}

This register is found at address $0 \times 1$, and defines the number of frequency increments in a single sweep. Since the register is 12-bit, the maximum is 4096 , while the minimum is 2 . This value depends on the resolution and maximum frequency required. Since the output frequency is defined as in Eq. 3.1, to give a maximum frequency of $58 \mathrm{kHz}$ with a $\Delta \mathrm{F}$ frequency of $3 \mathrm{~Hz}$ and an $\mathrm{F}_{\text {start }}$ of $52 \mathrm{kHz}$, the number of increments must be 2000. This value is transferred to address $0 \times 1$ with the data shown in Table 3.7 .

Table 3.7: Transferred data for the Number of Increments.

\begin{tabular}{|l|l|l|l|l|l|l|l|l|l|l|l|l|l|l|l|l|}
\hline $\mathbf{1 5}$ & $\mathbf{1 4}$ & $\mathbf{1 3}$ & $\mathbf{1 2}$ & $\mathbf{1 1}$ & $\mathbf{1 0}$ & $\mathbf{9}$ & $\mathbf{8}$ & $\mathbf{7}$ & $\mathbf{6}$ & $\mathbf{5}$ & $\mathbf{4}$ & $\mathbf{3}$ & $\mathbf{2}$ & $\mathbf{1}$ & $\mathbf{0}$ & Value \\
\hline 0 & 0 & 0 & 1 & 0 & 1 & 1 & 1 & 1 & 1 & 0 & 1 & 0 & 0 & 0 & 0 & $0 \times 17 \mathrm{D} 0$ \\
\hline
\end{tabular}

\subsubsection{Burst Interval}

The Burst Interval register at address 0x10xx defines the length of each burst in terms of time length or number of waveform cycles. Grimes et al [43] claims that maximum sensor response can be obtained with at least 80 cycles, so 80 cycles is used. The data are transferred to address $0 \times 10 \mathrm{XX}$, where XX means that it does not matter. The data are shown in Table 3.8 . 
Table 3.8: Transferred data for the Burst Interval.

\begin{tabular}{|l|l|l|l|l|l|l|l|l|l|l|l|l|l|l|l|l|}
\hline $\mathbf{1 5}$ & $\mathbf{1 4}$ & $\mathbf{1 3}$ & $\mathbf{1 2}$ & $\mathbf{1 1}$ & $\mathbf{1 0}$ & $\mathbf{9}$ & $\mathbf{8}$ & $\mathbf{7}$ & $\mathbf{6}$ & $\mathbf{5}$ & $\mathbf{4}$ & $\mathbf{3}$ & $\mathbf{2}$ & $\mathbf{1}$ & $\mathbf{0}$ & Value \\
\hline 1 & 0 & 0 & 0 & 0 & 0 & 0 & 0 & 0 & 1 & 0 & 1 & 0 & 0 & 0 & 0 & $0 \times 8050$ \\
\hline
\end{tabular}

\subsection{Power Amplifier}

The power amplifier is used to power the drive coil with enough current so that the sensor will produce a large enough signal in the reader coil. The input is taken from IOUT of the AD5930. Although in literature operational amplifiers have been used for this purpose [2], when this was attempted the signal from the sensor was too small, as the operational amplifier could not provide enough current to the coil.

Since the frequency of interest is in the $\mathrm{kHz}$ range, most IC audio power amplifiers can be used. The original power amplifier IC used in testing was a standard LM384 $5 \mathrm{~W}$ audio amplifier from National Semiconductor [44]. This was successful in driving the coil during the early stages in testing, but it was later decided that this chip's requirement of at least a $+12 \mathrm{~V}$ power supply was too high for portable systems.

For the above reason a smaller, lower power amplifier was in order; namely the SSM2211 1.5 W audio power amplifier from Analog Devices [45]. This is available as an 8-pin Small Outline Integrated Circuit (SOIC-8). This chip was chosen because of its low cost (approx. \$2NZ), relatively high power $(1.5 \mathrm{~W}$ ) and its $5 \mathrm{~V}$ power supply requirement. It also has adjustable gain by setting two external resistors, a feature not available on the previous LM384, which had a fixed gain of 50. In addition the chip has a SHUTDOWN mode, putting it in a low-power state, useful to save battery charge.

The SSM2211 has a differential output, so the output can swing to twice its supply voltage $(10 \mathrm{~V})$. One precaution shown in the datasheet was that when observing the output of the amplifier on an oscilloscope, it advised against connecting probes directly across a load, saying that this can short the outputs, causing damage to the chip. The datasheet therefore instructs the user to attach two probes on each side of the load and ground both, setting the oscilloscope to add the two channels (with one channel inverted). 


\subsubsection{Circuit Setup}

The schematic diagram for the power amplifier circuit is shown in Fig. 3.7. According to the datasheet, the voltage gain can be adjusted as:

$$
A_{v}=2 \times \frac{R_{F}}{R_{I}}
$$

Where $R_{F}$ is the feedback resistor $\mathrm{R} 32$ and $R_{I}$ is the input resistor R31. With the resistor values used the SSM2211 is configured for a voltage gain of $11.2 \mathrm{~V} / \mathrm{V}$. Since the output from the AD5930 is about $0.8 \mathrm{~V}$ peak-to-peak, the peak-to-peak voltage across the power amplifier's output is about $9 \mathrm{~V}$.

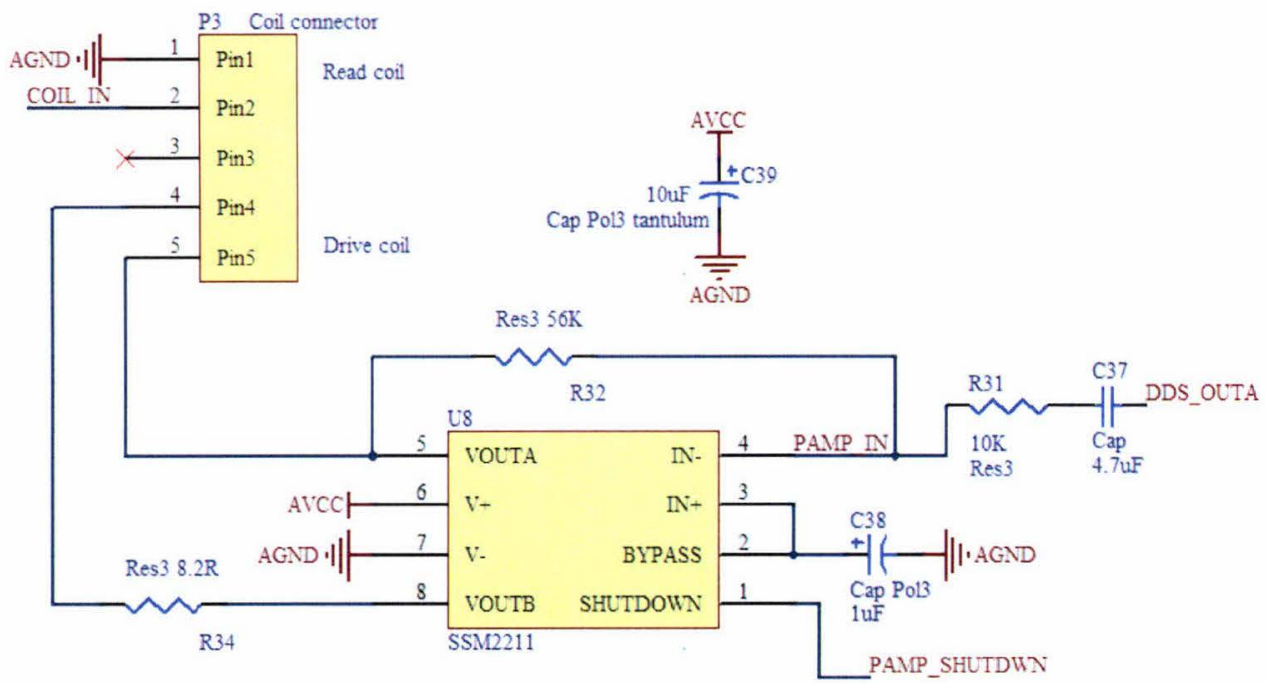

Figure 3.7: Schematic of power amplifier circuit with coil connector.

Another point to note is the relationship between the decoupling capacitor and input resistor. The datasheet states that the two components form a high-pass filter, blocking DC and low frequency signals. The relationship between cut-off frequency and the components is:

$$
f_{H P}=\frac{1}{2 \pi R_{I} \times C_{C}}
$$


predicting a high-pass cut-off of $3 \mathrm{~Hz}$. The SSM2211 also has a SHUTDOWN function at pin 1 . This is an active high signal which puts the power amplifier in a low power state. This pin is connected to the microcontroller's port 1, pin 2 .

The $8.2 \Omega$ resistor (R34) at the output of the power amplifier is present to protect the chip from damage as it was found that driving the coil without any resistance sometimes caused the chip to become very hot and fail. An $8.2 \Omega$ resistor was chosen as it is close to the resistance it is designed for ( $8 \Omega$ speakers). Using lower value resistors was found not to increase the signal quality, but it had the opposite effect where it caused more noise to be present in the reader coil.

Similarly to the AD5930, this IC is only available as SOIC-8 surface mount so during prototyping the SSM2211 was mounted on a simple PCB to interface with the breadboarded components.

\subsection{Operational Amplifier Circuits}

A single quad operational amplifier (op amp) was used for the four functions:

- Non-inverting amplifier (pre-amplifier)

- Low-pass filter

- High-pass filter

- Non-inverting amplifier (output amplifier)

The op amp chosen was an OPA4228 from Burr-Brown Instruments [46], a division of Texas Instruments. They are advertised as high precision, low noise op amps intended for a number of applications including filtering. They have a wide bandwidth $(33 \mathrm{MHz})$, high slew rate $(10 \mathrm{~V} / \mu \mathrm{s})$ and low noise $(3 \mathrm{nV} / \sqrt{\mathrm{Hz}})$ and cost approximately $\$ 28 \mathrm{NZ}$. They can be powered from supplies anywhere between $\pm 2.5 \mathrm{~V}$ to $\pm 18 \mathrm{~V}$ and so are very flexible. Both surface mount SOIC-14 and dual inline DIP-14 versions are available. The DIP-14 version was used so it could be easily replaced with a socket.

The first stage is the non-inverting amplifier. This amplifies the signal received from the reader coil, giving a voltage gain of about $30 \mathrm{~V} / \mathrm{V}$. The reason for having a voltage 
amplifier before the filter is to minimise noise; it was found feeding a small signal into would increase noise as the filter would not operate well enough.

The second stage is an active band-pass filter. The filter is needed because without filtering, the received signal looks like Fig. 3.8, with a lot of high frequency noise as well as low frequency ringing. This filter was designed as a Butterworth $2^{\text {nd }}$ order active filter, with cut-off frequencies at $72 \mathrm{kHz}$ and $41 \mathrm{kHz}$ for the low and high pass sections respectively. Since the signal is expected to be between $50 \mathrm{kHz}$ and $60 \mathrm{kHz}$, this is a sufficient range. The reason for a Butterworth filter was that it is the easiest to design, as well as have the flattest pass band response. Higher order filters were also investigated, but due to the higher part count and complexity they were not included in the final design.

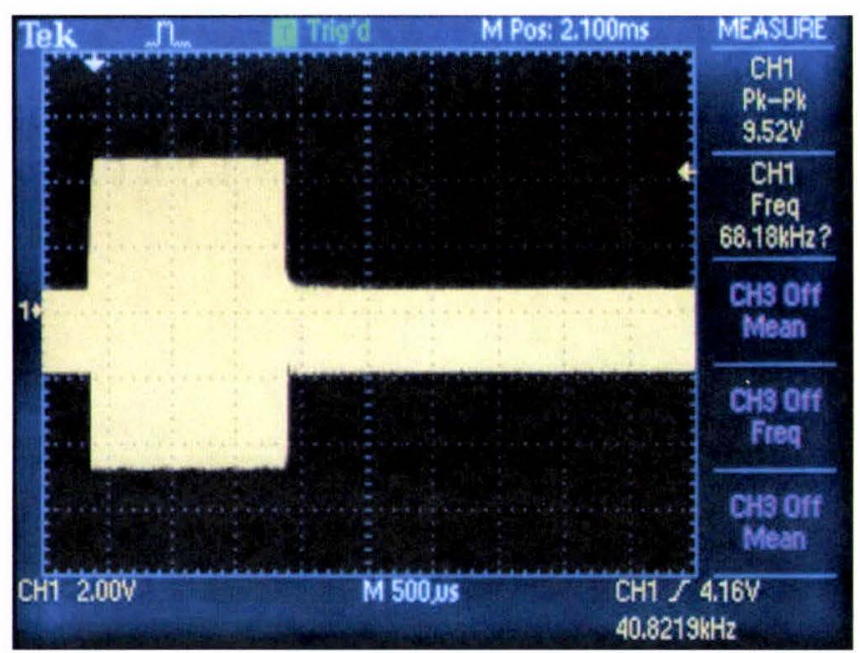

Figure 3.8: Amplified but unfiltered signal - due to noise we cannot see the sensor signal.

An important point found during testing was that the low-pass section of the filter must be put before the high-pass section, otherwise the output became unstable. This may have been due to the fact that there is a very large difference in signal amplitude between the drive and sensor signals. This then can cause the op amp output to hit the power supply rails when amplifying the drive signal, as the voltage gain needs to be quite large in order to amplify the sensor signal to detectable levels.

The final stage is another non-inverting amplifier to give the user control over the size of signal, as well as amount of noise. The gain adjustment is done manually with a 
single $10 \mathrm{k} \Omega$ potentiometer. Although a digital potentiometer could also be used for this purpose, it was not implemented for two reasons. Firstly it is expected that gain adjustment should only need to be done once, and that it would make the system unnecessarily more complex.

\subsubsection{Circuit Setup}

The designed op amp circuit showing the pre-amplifier, low-pass filter, high-pass filter and output amplifier is shown in Fig. 3.9.

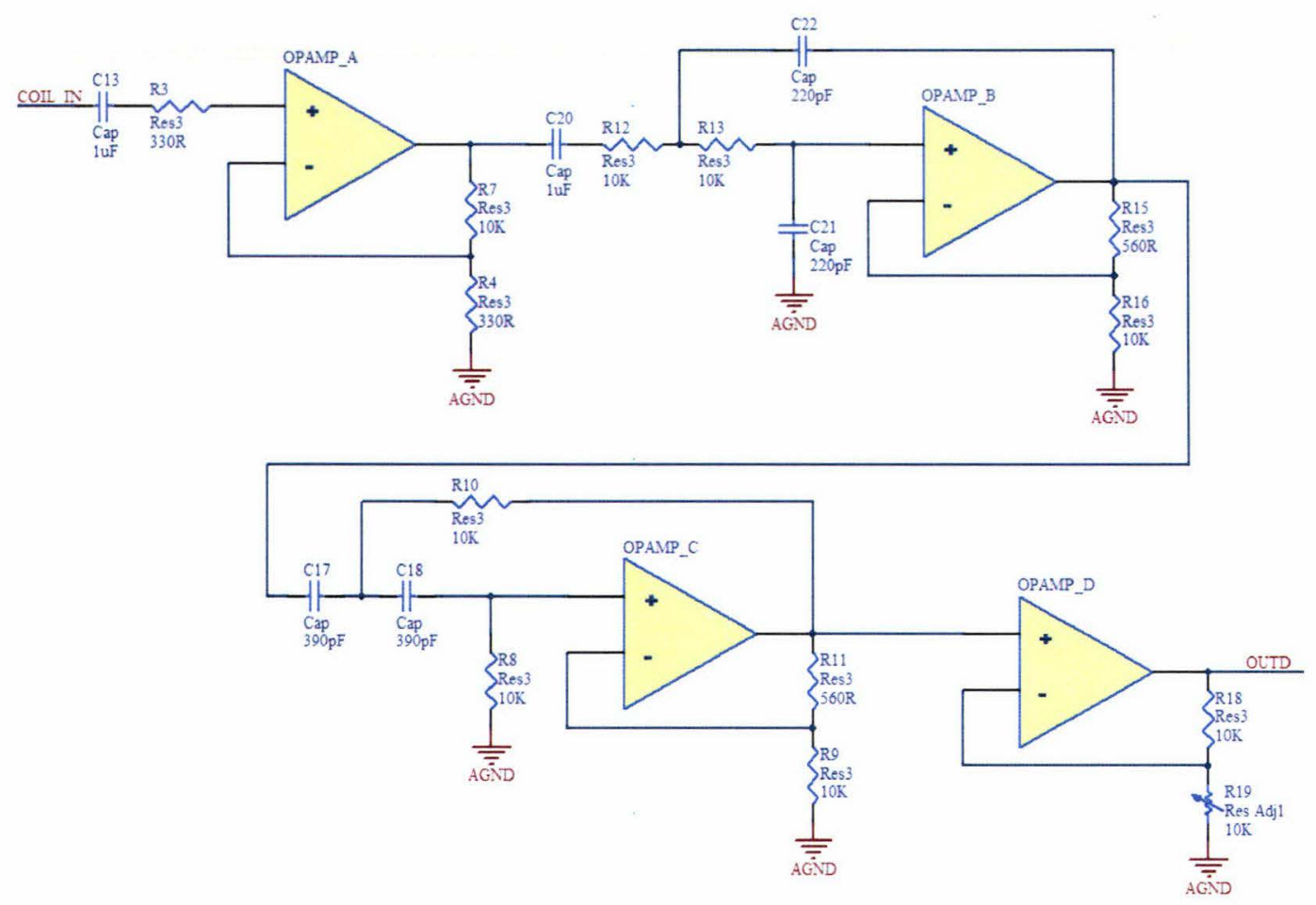

Figure 3.9: Schematic of op amp circuit.

\subsection{Voltage Comparator}

The voltage comparator is used to convert the amplified and filtered reader signal from the op amp to a series of digital pulses so that it can be read by the microcontroller's onboard counter. This operation is illustrated in Fig. 3.10. The comparator IC used was a standard LM311 (cost approx. \$1.50NZ) [47] due to its familiarity and simple operation. Similarly to the op amps, the LM311 is available in both SOIC-8 and DIP-8 versions. Again the DIP version was used for convenience. 


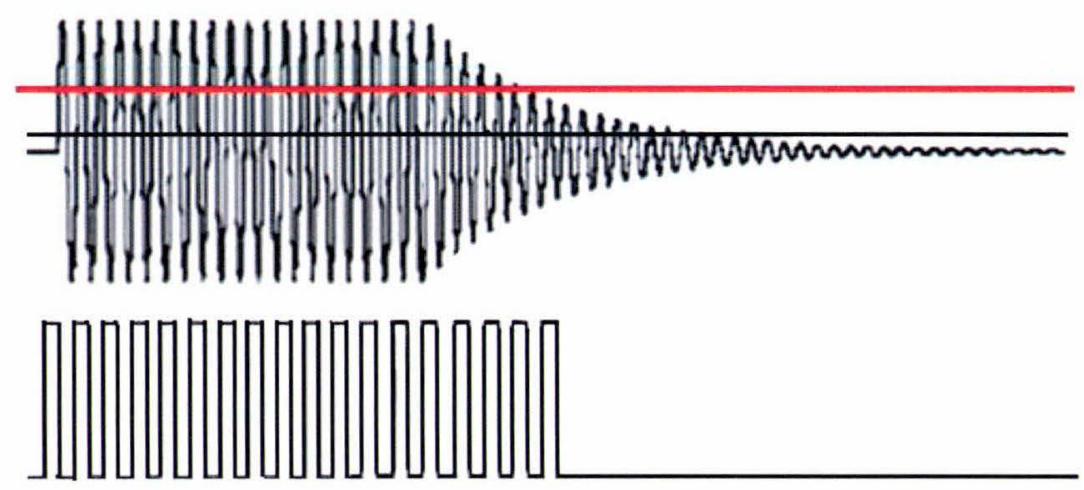

Figure 3.10: The voltage comparator produces a digital HIGH output (bottom) if the input signal (top) is higher than the reference voltage (red line).

\subsubsection{Circuit Setup}

The comparator circuit is shown in Fig. 3.11. The output of the fourth op amp (OUTD) from the previous section is fed into decoupling capacitor C25 to remove DC offsets, and then into the non-inverting input of the comparator. A hysteresis resistor R22 was also added but this was found to have little effect on the output.

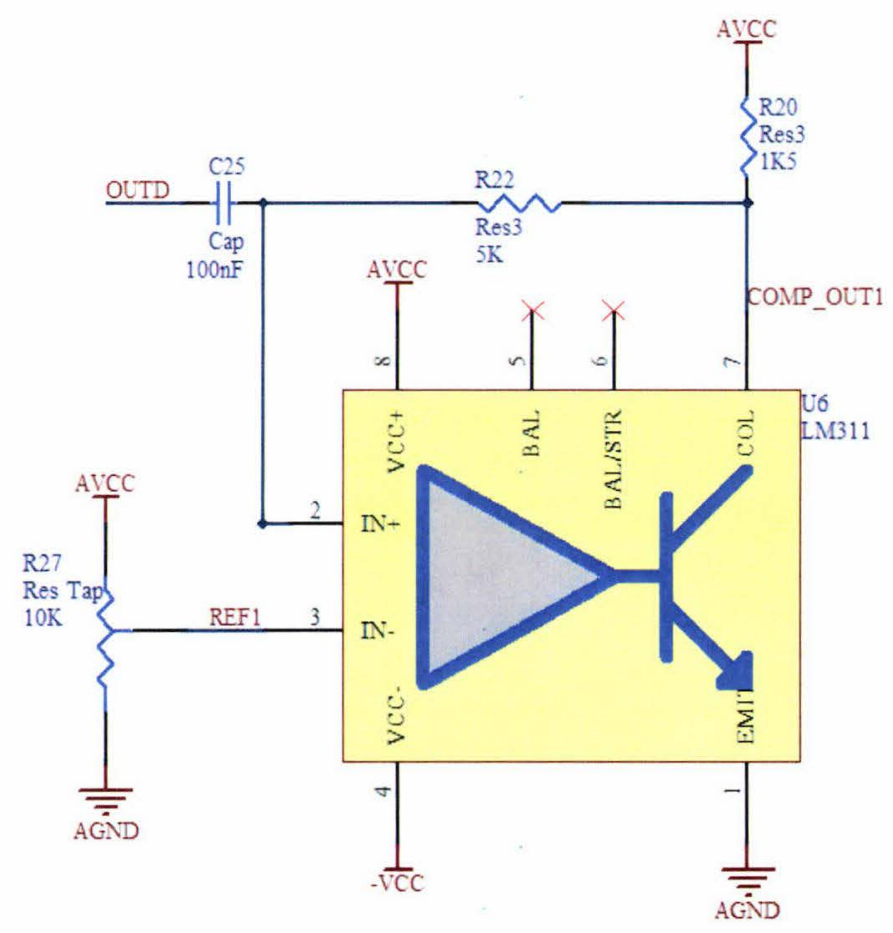

Figure 3.11: Schematic of voltage comparator circuit. 
The reference voltage is set with potentiometer R27, which acts as a voltage divider from $+5 \mathrm{~V}$ to ground. Nominally the reference voltage should be set to about $2 \mathrm{~V}$, with the noise floor (adjusted by the op amp output gain) set just below this level. The output is taken from the collector of the output transistor, giving a sharp pulse of 0 to $5 \mathrm{~V}$ when OUTD exceeds the reference voltage.

\subsection{Power Supply and Grounding}

The entire device is intended to be powered from a single $9 \mathrm{~V}$ battery or AC adapter, which is regulated to $5 \mathrm{~V}$ with a STMicroelectronics L4940D2T5 low drop-out (LDO) 1.5 A surface mount voltage regulator (cost approx. \$2.75NZ) [48]. Since this is an LDO regulator, the input voltage can be as low as $5.5 \mathrm{~V}$, which means lower voltage batteries and adapters can also be used if required.

Because the op amps and the voltage comparator require a negative supply, a Linear Technology LT1054CN8 voltage converter [49] chip is used to invert the $5 \mathrm{~V}$ into $-5 \mathrm{~V}$. The LT1054CN8 was chosen over other DC to DC converters because of its high current capability $(100 \mathrm{~mA}$ ), relatively low cost (approx. $\$ 8.40 \mathrm{NZ}$ ) and simple operation with minimal external parts required (just 3 capacitors). The package of the LT1054CN8 was chosen as DIP-8, so that it could be replaced easily with a socket.

The datasheet of the AD5930 recommends that the chip's analogue VCC (AVCC) and digital VCC (DVCC) supplies be separated in order to reduce noise and spurs. In the schematic for the chip's evaluation circuit, the DVCC was separated from AVCC through a bead inductor and a $1.5 \mathrm{k} \Omega$ resistor. This was attempted, but the voltage drop between the two power lines became somewhat large. Thus the series resistance was removed (see R30 on Fig. 3.6).

Another recommendation in the datasheet was to separate the analogue (AGND) and digital (DGND) grounds, and only make one connection between them with the ground link (see R29 in Fig. 3.6) being close to the AD5930. When this advice was ignored, the output became very noisy and spiky, and caused problems in other parts of the circuit. 


\subsubsection{Circuit Setup}

The power supply circuit is shown in Fig. 3.12, with the regulator on top while the voltage converter is found below. The voltage converter was found to have a voltage which was slightly higher than $-5 \mathrm{~V}$ at $-4.7 \mathrm{~V}$, possibly due to high current draw, when all the parts were powered from it. This was not a serious problem as the negative half of the signal waveform is not used for measurement.
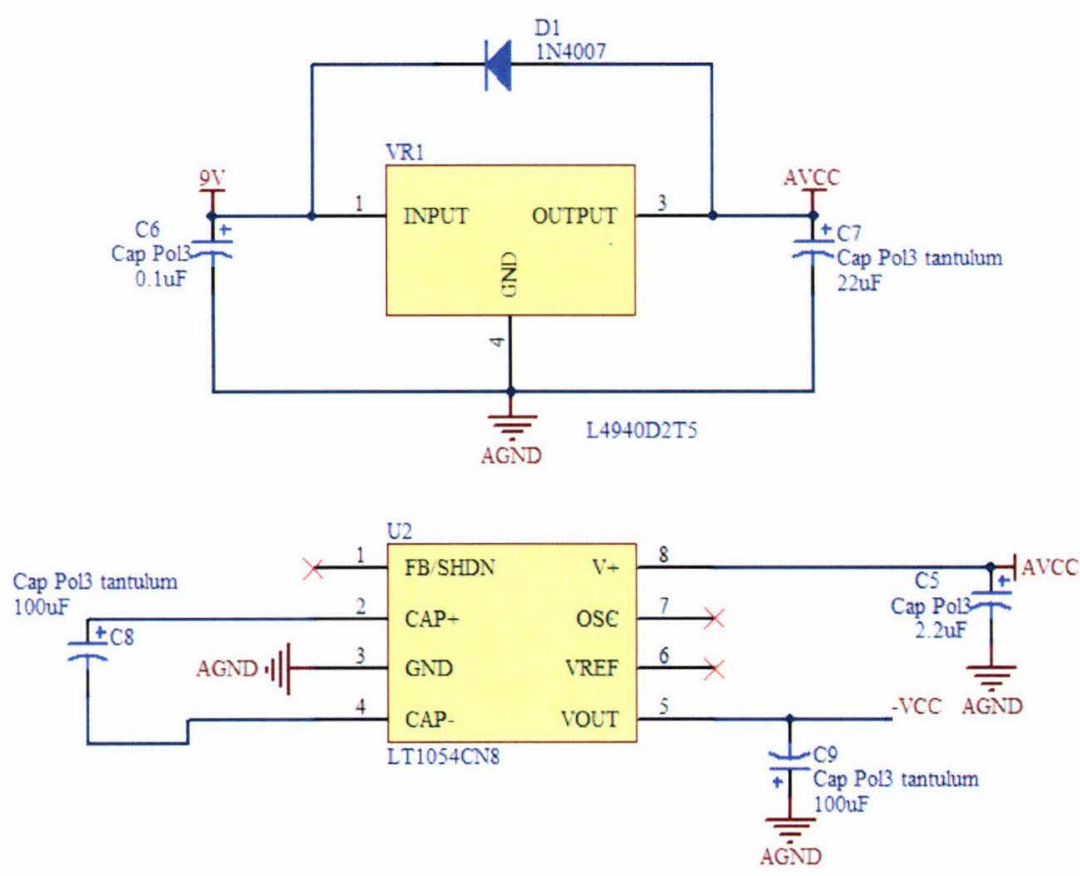

Figure 3.12: Schematic of power supply circuit. Shown on top is the $5 \mathrm{~V}$ regulator and bottom the voltage converter.

\subsection{Reader and Drive Coils}

The drive coil is connected to the output of the power amplifier, and creates the excitation AC magnetic field for the sensor. Put close to the sensor is the reader coil which receives the response of the sensor and feeds it into the first op amp. In some papers researchers have used a single coil for both functions [2] [43] by using a switching circuit; however to simplify the system this was not done in this case.

The coils were both designed as 40-turn, $8 \mathrm{~cm}$ diameter, air-cored types made from $0.5 \mathrm{~mm}$ enamelled copper wire. Larger diameter coils were investigated, but they 
tended to have more noise, and did not provide improvement as the signal was more difficult to restore. Coils with smaller diameters tended to have shorter range.

An important point is to provide spacing between the two coils. If the two coils are positioned close together, the reader coil had significant noise, and made the sensor signal undetectable. This is shown in Fig. 3.13 where the left image shows the reader signal without spacing and with spacing on the right. The spacing distance was set to $5 \mathrm{~cm}$ and this was found to be sufficient.

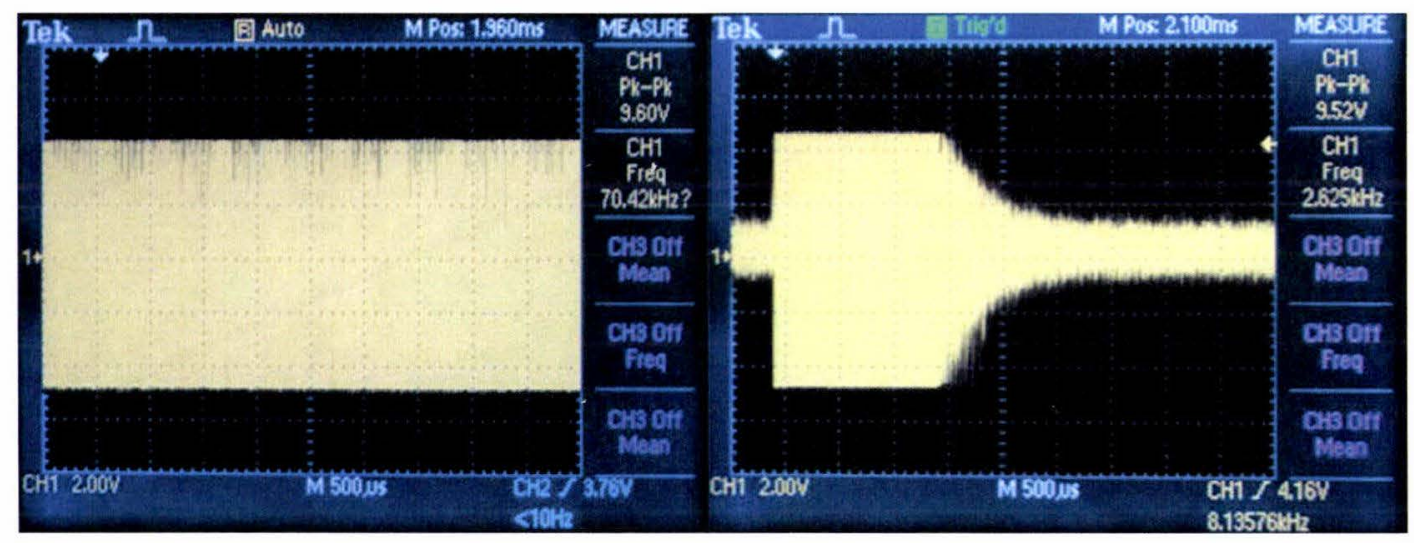

Figure 3.13: Difference in signal quality after filtering and amplification for 40 -turn reader and drive coils, not spaced (left) and spaced by $5 \mathrm{~cm}$ (right).

The final coils were made by wrapping the wire around a cylinder made from plastic and is shown in Fig. 3.14. Since the two coils are identical, they are interchangeable as long as the one closer to the sensor is used as the reader.

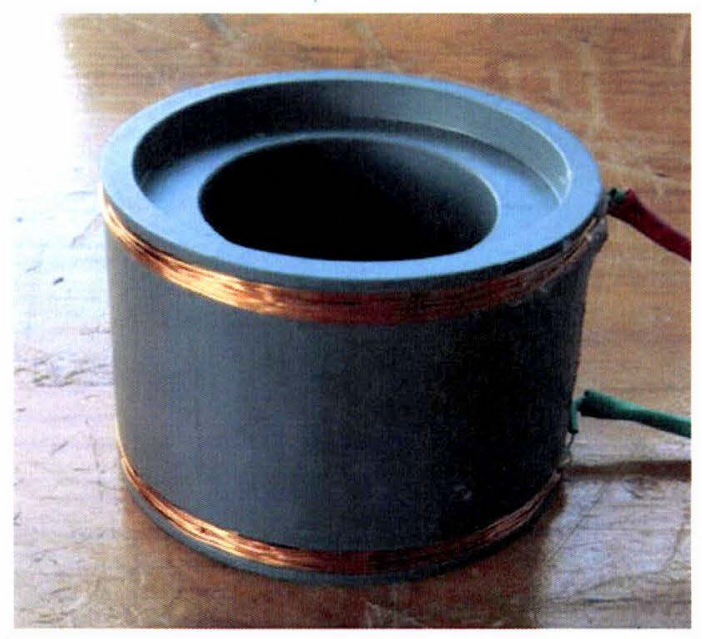

Figure 3.14: Reader probe consisting of the drive coil and reader coil. 
Space for a $7.5 \mathrm{~cm}$ diameter, ring shaped magnetic core is also provided on both sides for further upgrades in the future if required. The total weight of the probe was measured to be about $270 \mathrm{~g}$.

During testing, it was found that the probe is highly sensitive to interference from CRT screens, especially if positioned perpendicular to the screen. The interference caused significant noise to be present in the reader signal, making the reader unusable. To prevent such noise problems it is therefore recommended that when the reader is in use, the probe be placed at least $2 \mathrm{~m}$ away from CRT screens, or even turn these screens off.

\subsection{Printed Circuit Board Design}

The PCB for the reader system was designed in Altium Designer as a doubled sided copper board. The designed board is shown in Fig. 3.15. The PCB was designed to be installed in a Hammond 1599EBK handheld plastic case. This case was chosen due to its ideal size (height $\times$ length $\times$ width $=35 \times 170 \times 85 \mathrm{~mm}$ ) and low cost (approx. $\$ 20 \mathrm{NZ})$.

In order to able to fit the $\mathrm{PCB}$ in the case and to save space, surface mount resistors and capacitors were used. The package types of resistors and capacitors were chosen as $0805(0.08 \times 0.05$ inches $)$ as they were the most common with distributors, meaning they are the easiest to find. The trimming potentiometers were also made surface mount. Tantalum capacitors were placed near each IC to filter the power supply, and these were chosen to be case-C surface mount.

For integrated circuits which have both DIP and SOIC versions, their DIP versions were used, as they could be replaced easily with a socket, and that the same parts used on the breadboard could be used. This eliminated the need to find and order their surface mount counterparts.

The reader and drive coils are connected to the PCB with a 5-way terminal block. Viewed from the front, the 2 connectors on the left hand left side is the output from the power amplifier for the drive coil, and the 2 connectors on the right hand side are 
the input and ground lines from the reader coil. The central connector is not connected. The terminal block has a sticker with " $\mathrm{R}$ " and " $\mathrm{D}$ " to indicate the connections as shown in Fig. 3.16.

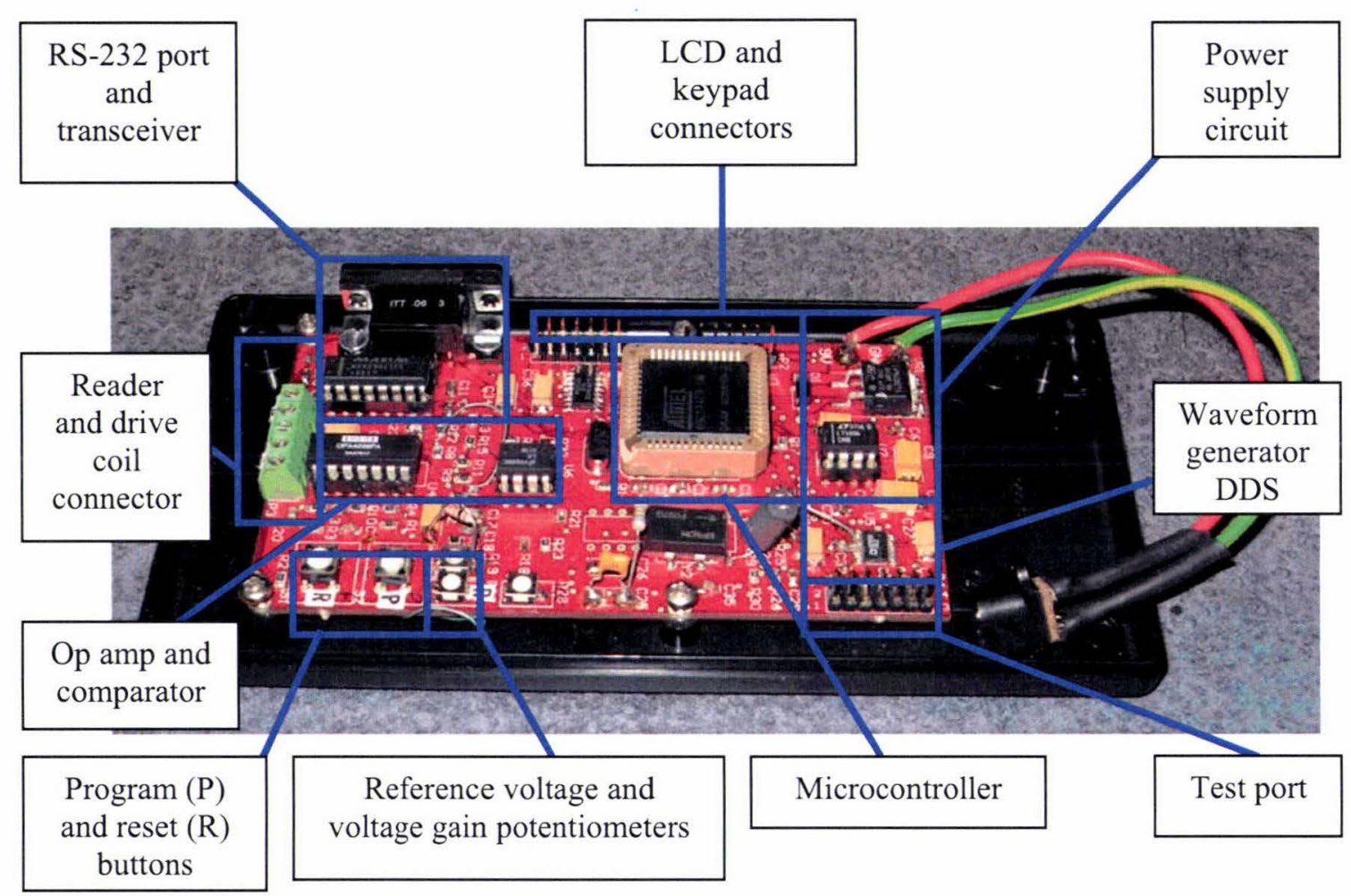

Figure 3.15: Completed PCB of reader mounted in its case.

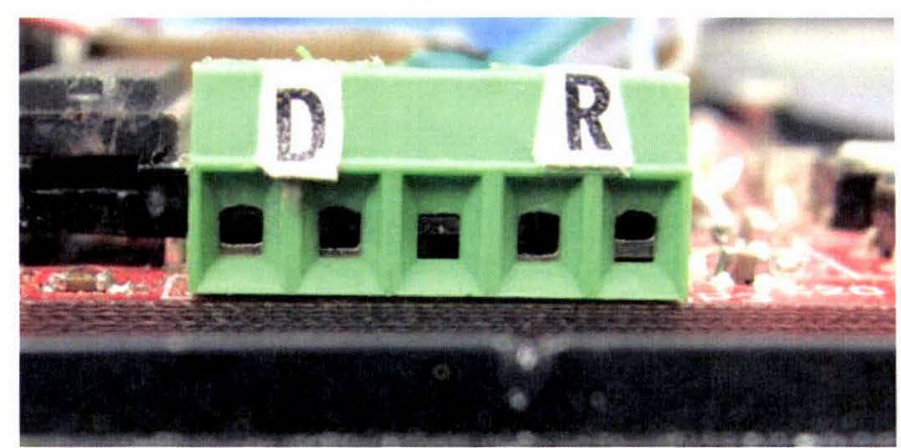

Figure 3.16: Terminal block connector for the reader and drive coils.

As the datasheet for the AD5930 suggested, the board is separated into analogue and digital sections; the digital circuitry (microcontroller, RS-232 etc.) are shielded with digital ground planes, while analogue circuitry (op amps, comparator etc.) are given analogue ground planes. 
To aid in debugging and testing the board, a test port was added on the top right hand corner of the PCB which has connections to some of the major signal and power lines. The layout of the test port is shown in Table 3.9. Note that pins 10 and 12 are not used; they were originally intended to test the second voltage comparator to add further functionality, but it was decided it is not necessary to have two comparators.

Table 3.9: Test port layout on the PCB.

\begin{tabular}{|c|c|c|}
\hline Pin no. & Connection & Function \\
\hline $\mathbf{1}$ & DVCC & Digital +5 V power line \\
\hline
\end{tabular}


Finally, through switching to surface mount components as opposed to through-hole used in testing for the op amp circuits, the response of the filter and non-inverting amplifier was worse, with significant being noise present in the output signal, limiting the detection range. Feeding sine waves of varying frequencies from a function generator into the filter showed that the circuit was operating correctly without significant noise being present. Only when the actual measurement signal which is of varying frequency and amplitude was being fed was the output problematic. This therefore may have been due to an issue with the choice of capacitors used in the filter.

After some time looking at methods to solve this problem, it was decided to construct an external op amp circuit using only through-hole components. The PCB facility at university could not be used at this time due to technical problems with the PCB milling machine, so a prototyping board was used. In this board, instead of using a single OPA4228 quad op amp, two OPA2228 dual op amp chips (cost approx. $\$ 11.50 \mathrm{NZ}$ ) were used to allow more space for wiring.

This new board was found to operate significantly better, with less noise and better stability. The board is shown in Fig. 3.17, and Fig. 3.18 shows how it is installed inside the case. The op amp board is placed on top of the original reader PCB, and the case is closed on top. A piece of anti-static foam is used to separate the op amp board from the original reader PCB. The op amp circuit built on the original PCB is bypassed, and the input and power lines for the board is taken from the IC socket for the OPA4228 op amp which was previously installed on the PCB. The input (yellow wire) is plugged into the pin 3 of the socket, while the $+5 \mathrm{~V}$ (red wire) and $-5 \mathrm{~V}$ (green wire) power lines are plugged into pin 4 and pin 11 pins of the socket respectively. The ground is soldered to an available ground point, while the output is soldered to pin 2 of the LM311 comparator. 


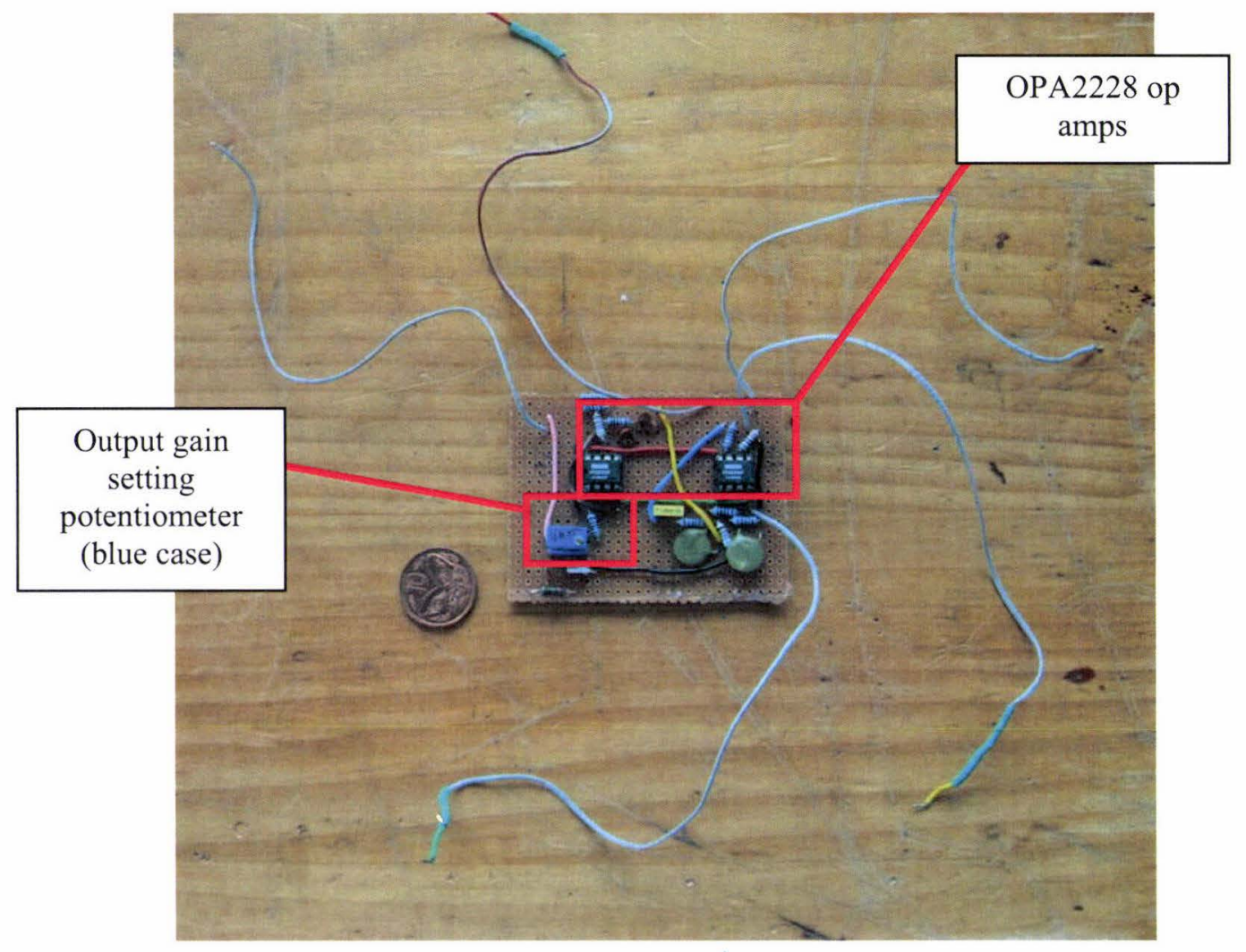

Figure 3.17: Constructed op amp circuit with a prototyping board.

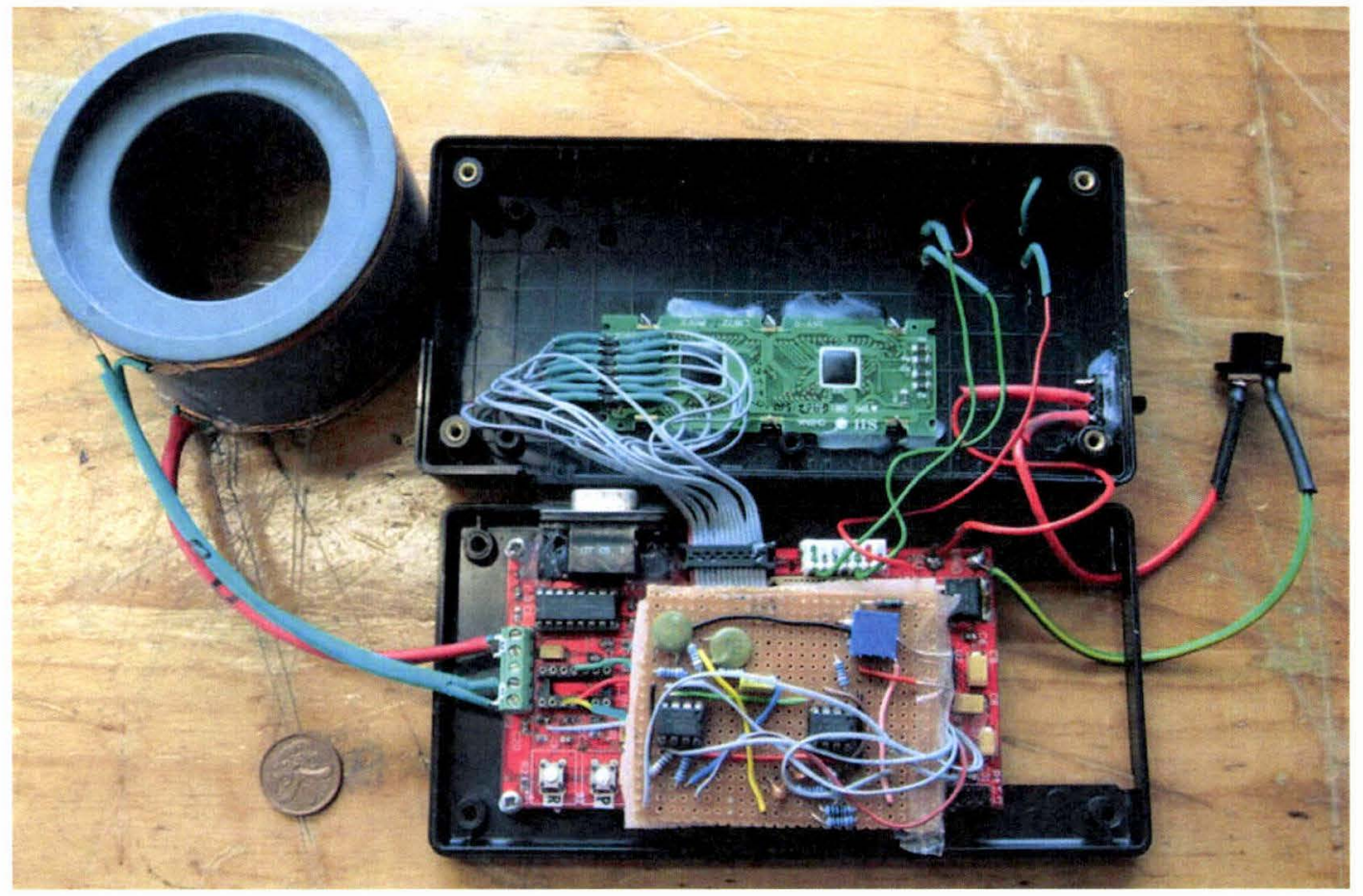

Figure 3.18: Completed reader system with case open, showing the reader probe and the new op amp board, which is mounted on top of the original PCB. 


\subsection{Completed System}

The reader system with the cover on is shown in Fig. 3.19. With all the parts installed in the case, the total weight of the system was approximately $250 \mathrm{~g}$ ( $520 \mathrm{~g}$ including reader probe), which is very reasonable for handheld use. An approximated cost of the reader is $\$ 200 \mathrm{NZ}$ including parts and labour. A Single Pole Double Throw (SPDT) slide switch was added to the side of the case for ON/OFF control. This should prove useful when a battery is used to power the system.

At the lower right hand corner are the pushbuttons for user input. The connection for the buttons was explained in Section 3.4.2. The green button is the "RUN" (button 1) button, and is used to confirm user input and start measurements, and red is the "STOP" (button 2) button which stops a measurement if it is held down for about 2 seconds.

In addition to the above, two small holes were drilled above the PROGRAM and RESET buttons on the PCB, and this allows the system to be programmed from PC without opening the case. These buttons were placed internally to simplify the user interface as well as to reduce cost - the smaller internal buttons were much cheaper than larger external buttons (approx. $\$ 2.50$ vs. $\$ 6.80$ ). Since these buttons are only needed when reprogramming the microcontroller, they should not cause significant inconvenience to the user.

After connecting the serial cable to the RS-232 port, the user can put the microcontroller into programming mode by holding the PROGRAM button down, and then pushing the RESET button with a pair of thin sticks (such as a screwdriver) as shown in Fig. 3.20. The device can now be connected and programmed though FLIP. 


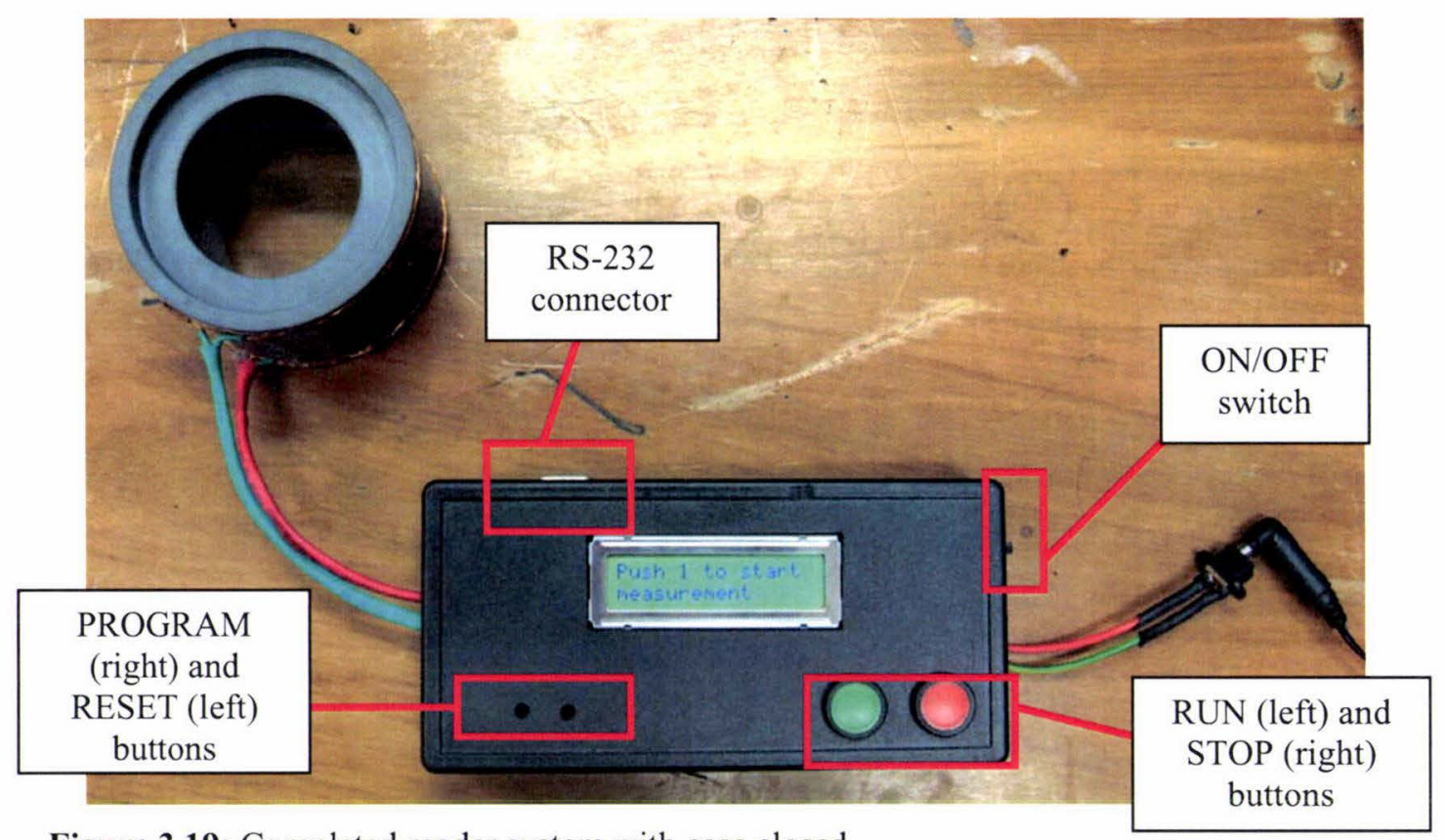

Figure 3.19: Completed reader system with case closed.

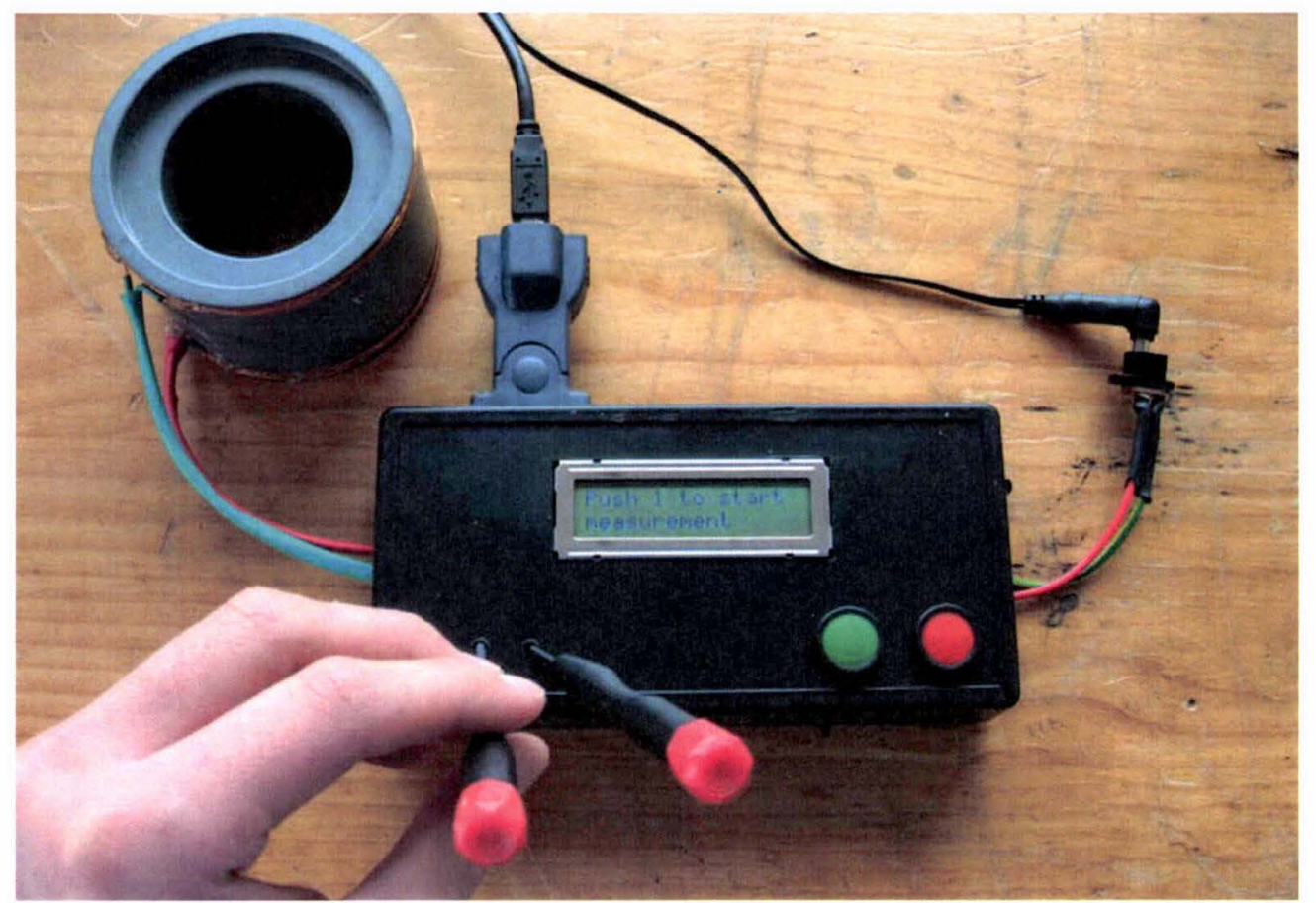

Figure 3.20: Setting the reader into programming mode by pushing the two internal buttons.

\subsection{Performance of System}

The performance characteristics of the sensor reader was evaluated by measuring the resonant frequency of a generic magnetoelastic sensor, designed to resonate at about 
$54 \mathrm{kHz}$. The firmware code of the microcontroller was slightly modified to display the number of sensor pulses detected as well as the measured resonant frequency at the end of the measurement.

The sensor was placed at different positions from the reader probe and the number of detected pulses from the sensor and the resonant frequency measured was recorded. 10 measurements were done at each position to calculate the mean value and to find the spread in values.

\subsubsection{Detection Distance}

The reader probe was put at different distances to the magnetoelastic sensor, and the performance of the system was tested. The probe was put on its side, and the sensor was aligned to the centre of the reader coil. The distances were measured from the edge of the reader probe to the end of the sensor strip. The placement of the probe and sensor is shown in Fig. 3.21.

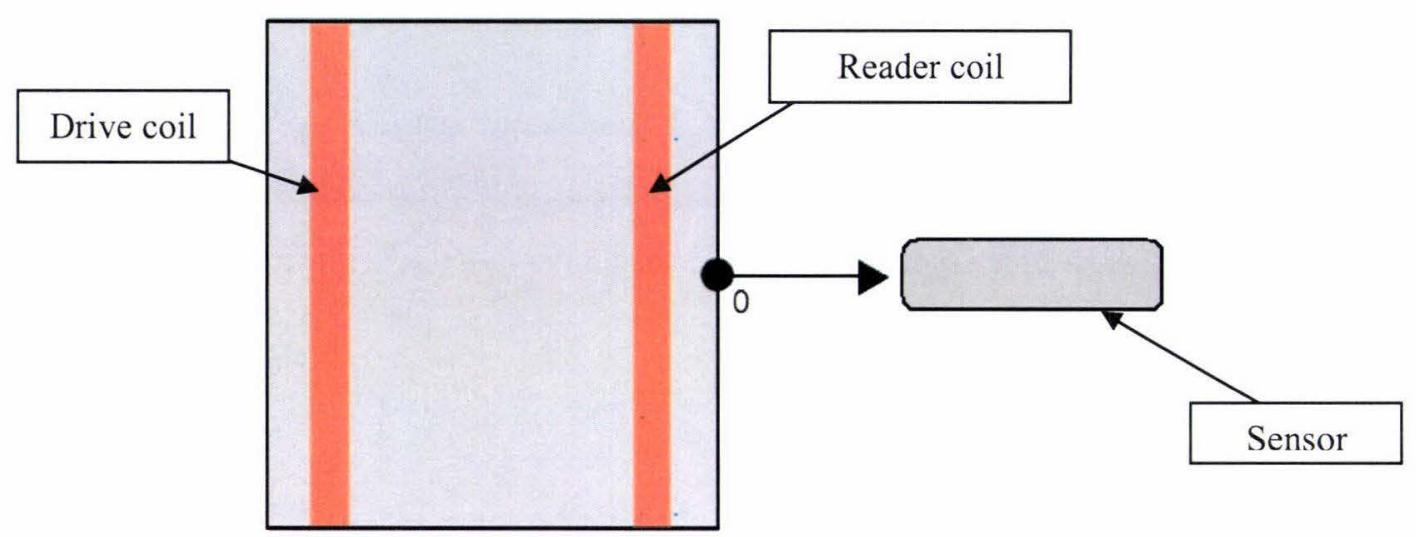

Figure 3.21: Measuring the effect of distance from reader coil.

The plot of the number of sensor pulses detected against distance is shown in Fig. 3.22. It was found that the reader is able to detect the sensor up to $2 \mathrm{~cm}$ away. If it is put further away, then the number of detected sensor pulses became less than the threshold value of 40, and was no longer detected. The drop in number of detected sensor pulses against distance was approximately linear with a gradient of -18.6 pulses $/ \mathrm{cm}$. The standard deviation of the number of sensor pulses detected was between 1.1 and 1.9 . 


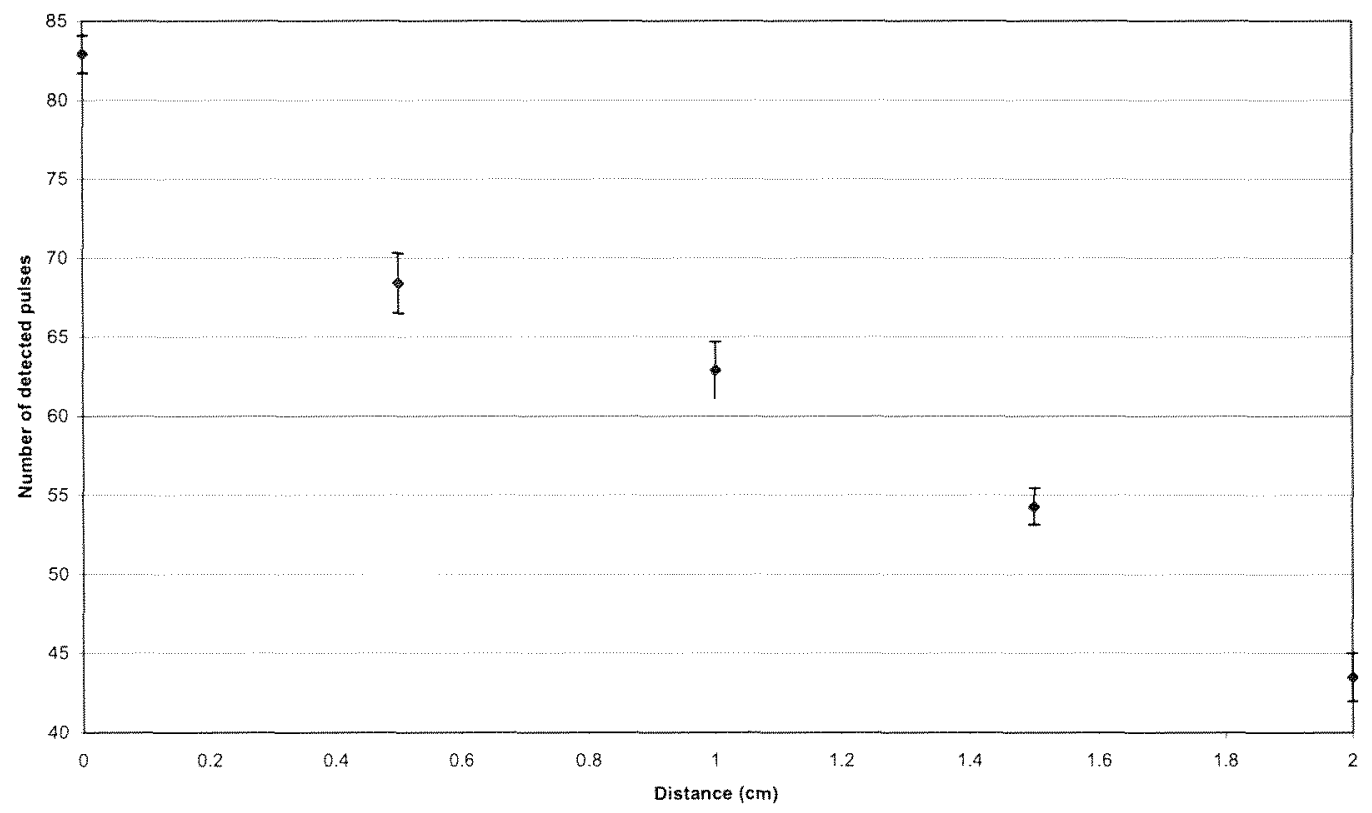

Figure 3.22: Distance from reader probe versus mean number of detected sensor pulses, also showing the standard deviation.

The following plot of Fig. 3.23 shows the mean of the resonant frequencies of the sensor measured by the reader, as well as the standard deviation at each distance.

Fig. 3.23 shows that the mean in measured frequencies varied from $54.24 \mathrm{kHz}$ to $54.33 \mathrm{kHz}$, but an obvious relationship with distance could not be observed. Fig. 3.23 also shows that placing the sensor at further distances does not increase the standard deviation (spread) in measured resonant frequency significantly, although at $2 \mathrm{~cm}$ the standard deviation was largest. 


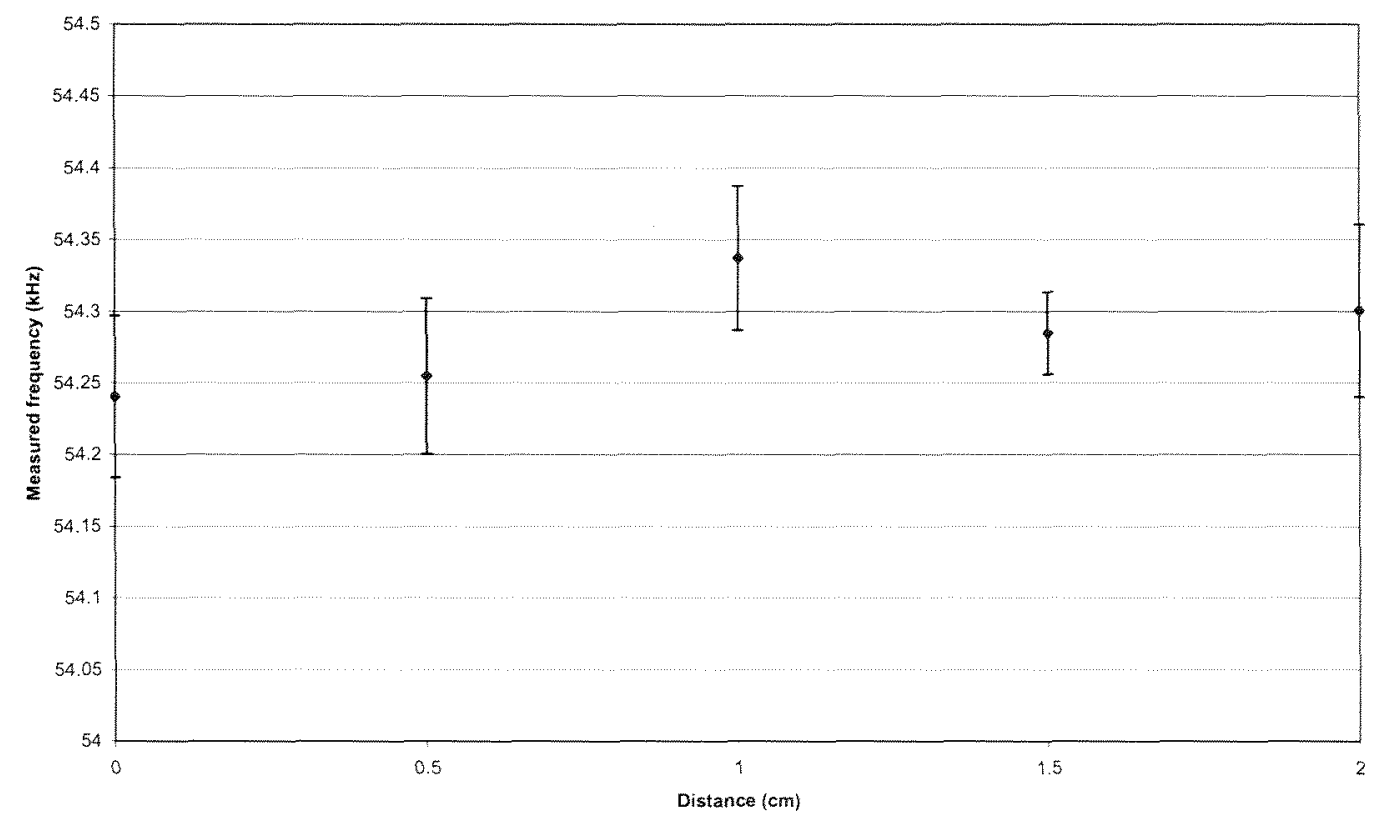

Figure 3.23: Distance from reader probe versus mean of measured resonant frequencies, also showing the standard deviation.

\subsubsection{Offsetting from Centre}

The effect of misalignment from the centre of the reader coil was investigated. The end of the sensor was placed at a constant distance $(0 \mathrm{~cm})$ from the end of the reader probe but the position of the sensor was offset from the centre of the probe by varying distances. Fig. 3.24 shows how the sensor and reader probe were positioned.

This offset distance was varied from $0 \mathrm{~cm}$ (centre of the coil) to $3 \mathrm{~cm}$ (just inside the coil). At offsets over $3 \mathrm{~cm}$ the number of sensor pulses detected became less than the threshold of 40 and were not detected. The standard deviations of the number of pulses was similar to that in Section 3.13.1, and were found to be between 1.1 and 1.9 and are indicated by the error bars on Fig. 3.25. 


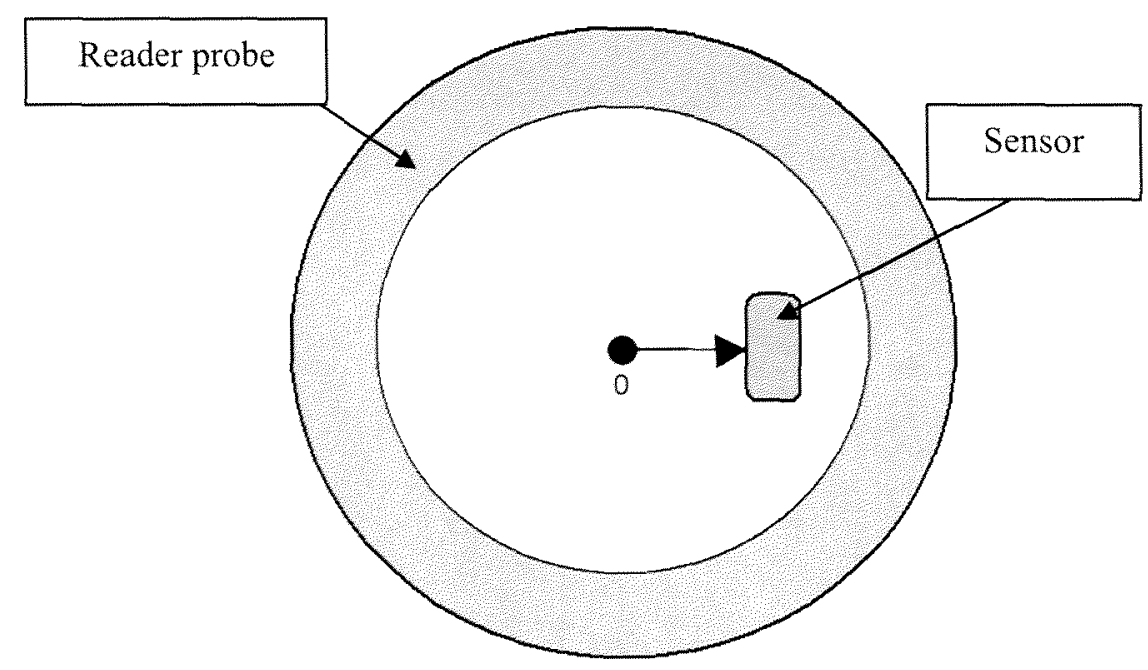

Figure 3.24: Measuring the effect of offsetting from the centre of the reader coil.

The plot of the number of sensor pulses detected against distance is shown in Fig. 3.25. The drop in the number of detected sensor pulses against offset distance was approximately linear with a gradient of -9.58 pulses $/ \mathrm{cm}$. The plot of Fig. 3.26 shows the mean of the measured resonant frequencies of the sensor as well as the standard deviation at each offset distance.

Fig. 3.26 shows that the average in measured resonant frequencies do not change significantly with increased offset distance, with the values ranging from $54.20 \mathrm{kHz}$ to $54.26 \mathrm{kHz}$. Fig. 3.26 also shows that the spread in measured frequencies tends to increase with increased offset distance. This suggests that in order to make the measurement to be most accurate and reproducible, the sensor should be placed near the centre of the reader probe. 


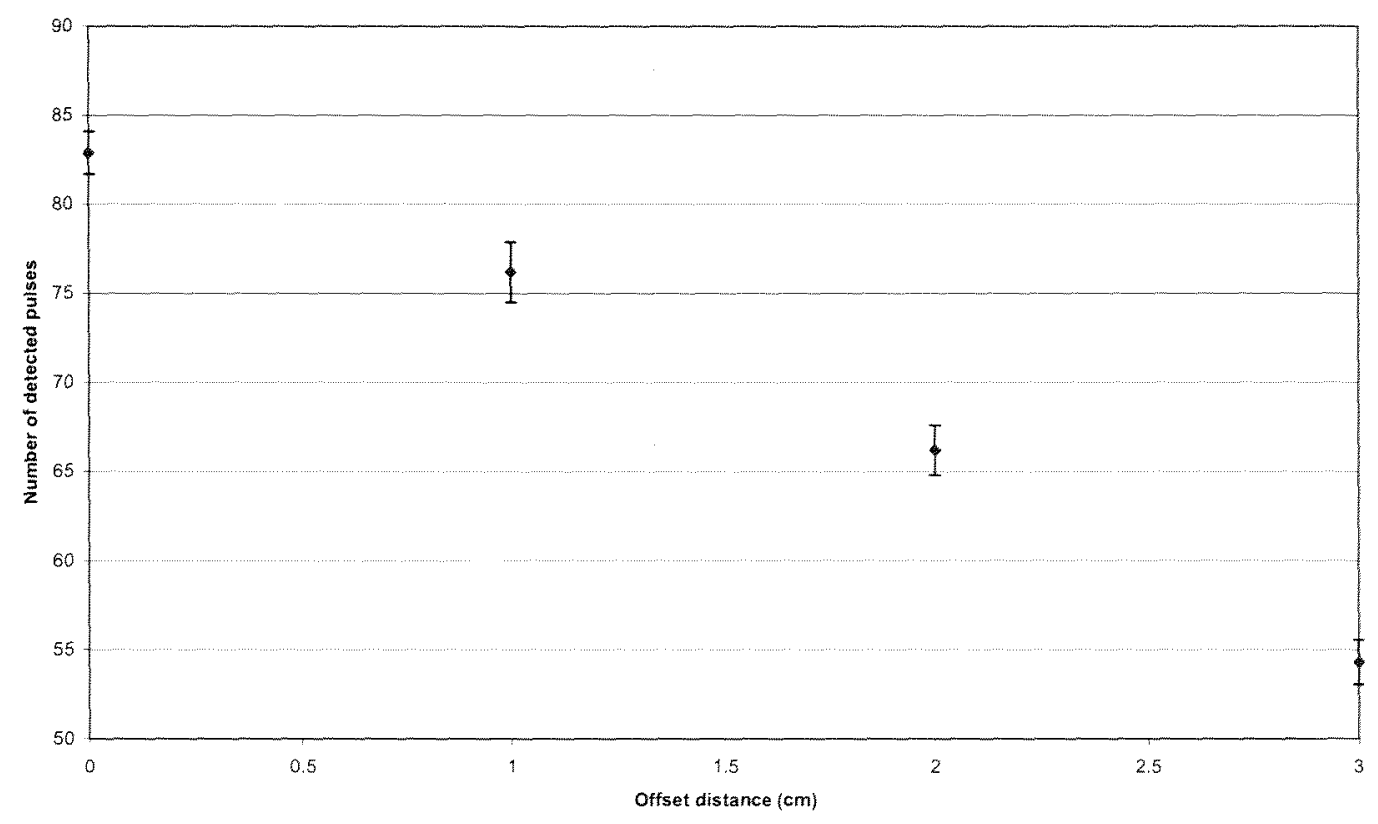

Figure 3.25: Offset distance from centre of reader probe versus mean number of detected sensor pulses, also showing the standard deviation.

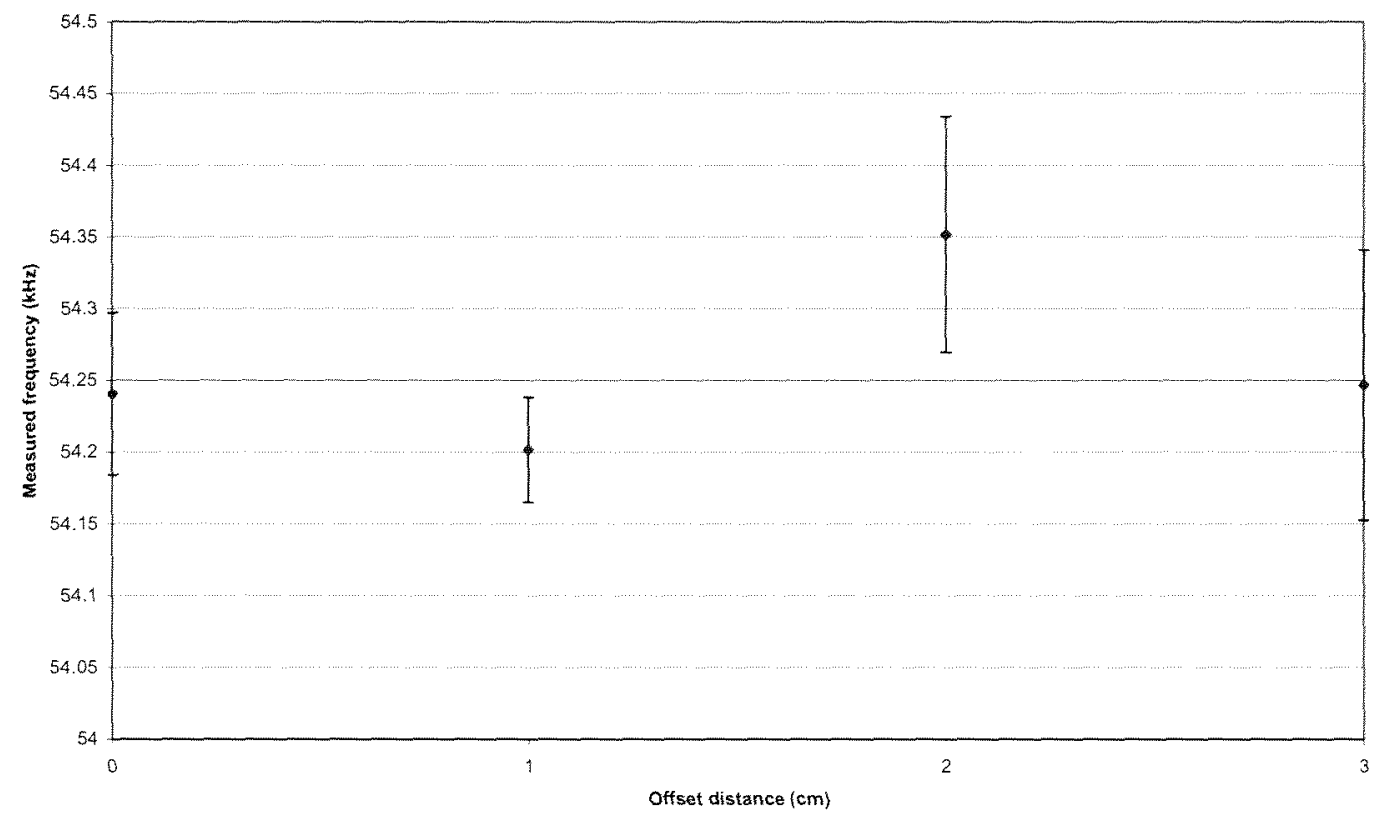

Figure 3.26: Offset distance from centre of reader probe versus mean of measured resonant frequencies, also showing the standard deviation. 


\subsection{Possible Improvements}

There are a number of possible improvements that can be made to the reader. One major point is to improve the detection distance. Currently the detection limit is only $2 \mathrm{~cm}$ - this is limited mainly by noise issues in the input signal. If the signal to noise ratio can be improved the signal amplitude can be increased and thus achieve a better detection distance. A larger reader coil may also be necessary.

As explained in Section 3.10 there is a space for a magnetic core in the reader probe. The effect of adding a core could be investigated. This may increase the signal strength and hence increase the detectable distance. A few adjustments to the detection circuitry may also be necessary.

Another possible area is to integrate the reader and drive coils into the PCB by employing a spiral inductor, rather use an external coil pair. This would further improve the portability and robustness of the system by having just one box to carry.

The RS-232 port of the reader is currently only used when programming the microcontroller. This port can also be used to interface with software on PC, and this means that PC software can be designed to add further functionality to the system.

Another area is to make measurements faster. Currently the system takes about 1.5 minutes to complete a measurement, which can be a bit slow. As stated previously, simply employing a faster microcontroller would not improve measurement speed due to the waiting required at each frequency. One solution would be to reduce the number of frequencies that are analysed in the measurement; however this could result in reduced accuracy in the resulting resonant frequency. 


\section{The Development of Magnetoelastic Sensors}

\subsection{Introduction}

In Chapter 2, some selected properties of magnetoelastic materials were discussed. One of these properties was the requirement for a DC biasing magnetic field. It was explained that a DC magnetic field applied longitudinally to the sensor strip increases the signal of the sensor, as well as shifts its resonant frequency. In Section 4.2, these effects were investigated by placing the strip in different magnetic fields provided by a Helmholtz coil pair.

In order to make the sensor small and portable, the bias field was to be supplied by a magnet, attached to the sensor strip so as to give the required field. Section 4.3 discusses the use of a permanent magnet for biasing.

Also in Chapter 2, the temperature dependence of magnetoelastic materials was discussed. Section 4.4 shows the measurements performed to investigate the temperature dependence of Metglas 2826MB strips.

Sections 4.5 and 4.6 explain the work done to make the Metglas strip sensitive to humidity. This was achieved by applying water vapour absorbent materials to the Metglas strips. Two different coatings were tested - polypyrrole (PPy) and polyvinyl alcohol (PVA). Testing of the coated sensor strips is discussed in Section 4.7.

Section 4.8 brings together the results of the previous sections to construct a prototype system for humidity measurement. The reader constructed in Chapter 3 was used to perform practical measurements in a sealed food container and a wall cavity. Section 4.9 discusses the long term stability of the constructed humidity sensor.

Finally in Section 4.10 a method to construct a small sensor array using the Metglas $2826 \mathrm{MB}$ material is discussed, and characteristics of the constructed sensor array are described. 
The Metglas 2826MB magnetoelastic sensor strips used were provided to SCPS as samples from Metglas Inc.

\subsection{Experimental Arrangement and Procedure for Impedance De-tuning}

The experimental arrangement used for impedance de-tuning measurements on magnetoelastic sensors is shown in Fig. 4.1. This experimental arrangement consisted of a Topward 6303D DC power supply (1), supplying current to a Helmholtz coil (2),

a $5 \mathrm{~cm}$ diameter reader coil with approximately 70 turns (3), connected to the terminals of an Agilent HP4294A impedance analyser (4), and the Metglas strip (5) which was $40 \mathrm{~mm} \times 13 \mathrm{~mm}$ in size, was placed on a flat plastic bar inside the reader coil.

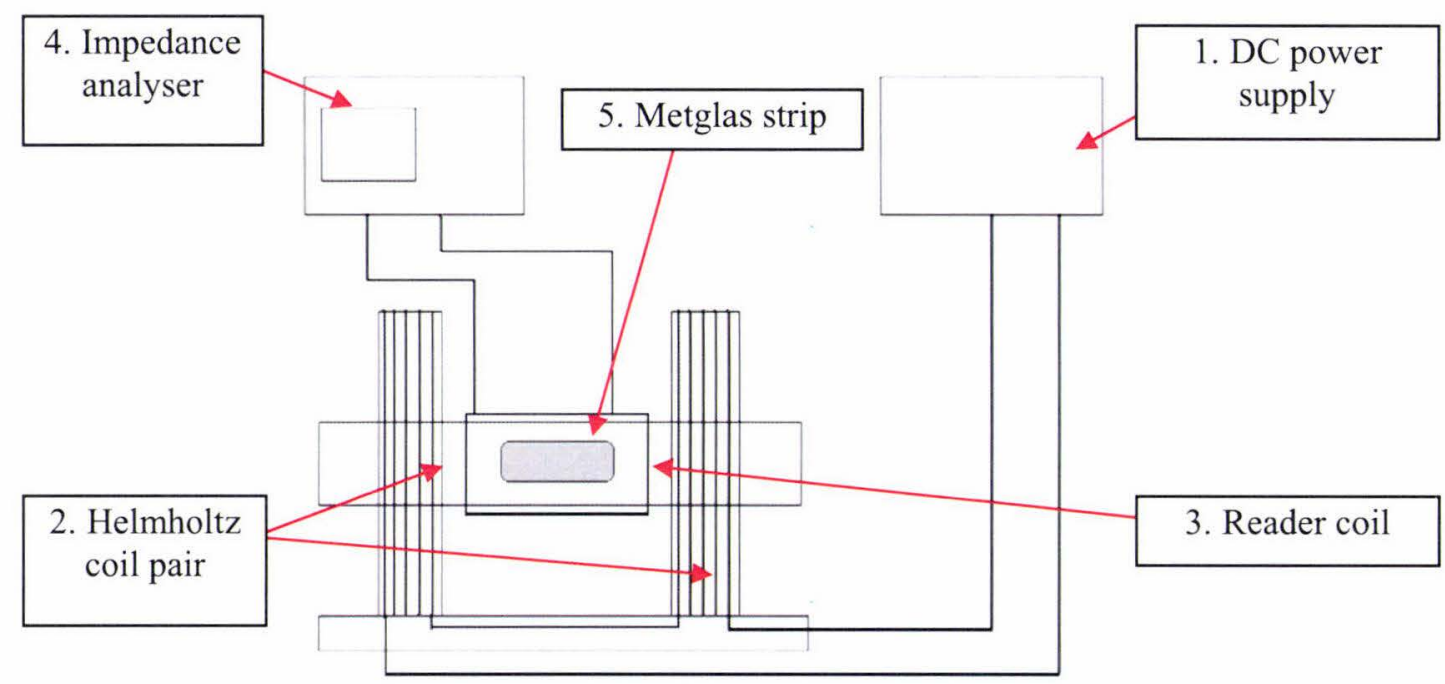

Figure 4.1: Experimental arrangement for impedance de-tuning.

A Lakeshore 421 gaussmeter was used to calibrate the Helmholtz coil between current and magnetic field strength. The probe was placed in the centre of the coil, and the field was measured while the current was varied. The Helmholtz coil used had the current-magnetic field strength characteristic shown in Fig. 4.2. 


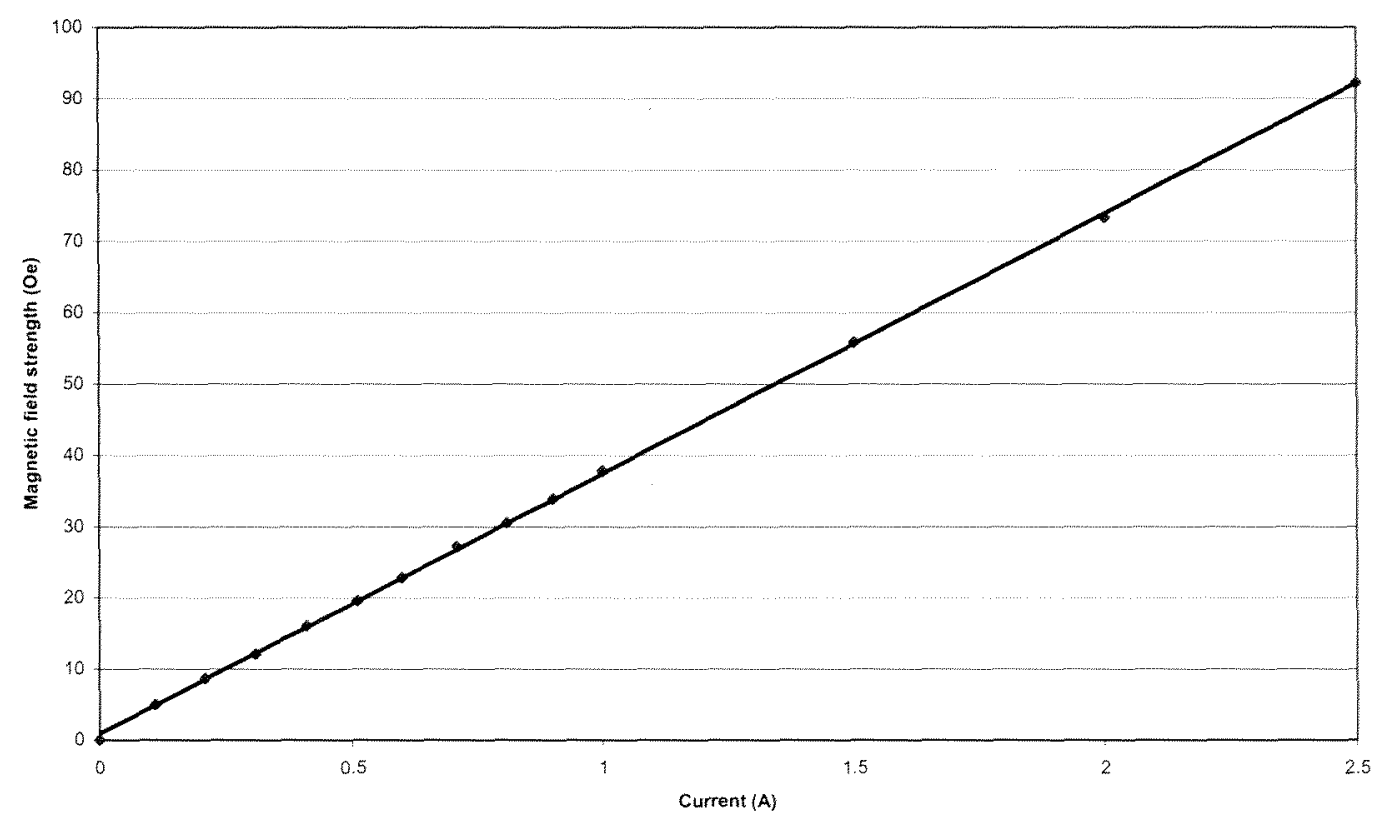

Figure 4.2: Current versus magnetic field strength for the Helmholtz coil.

The field strength was linear with current, and mathematically the relationship was:

$$
H=37.139 I
$$

Where $H$ is the magnetic field strength in Oe, and $I$ is the current through the coil in A. In previous studies [5] this Helmholtz coil arrangement showed good uniformity in magnetic field strength, with the field strength varying by no more than $5 \%$ over the length of the $40 \mathrm{~mm}$ Metglas ribbon.

Fig. 4.3 shows the relationship between relative field strength versus the position of the sensor strip. 


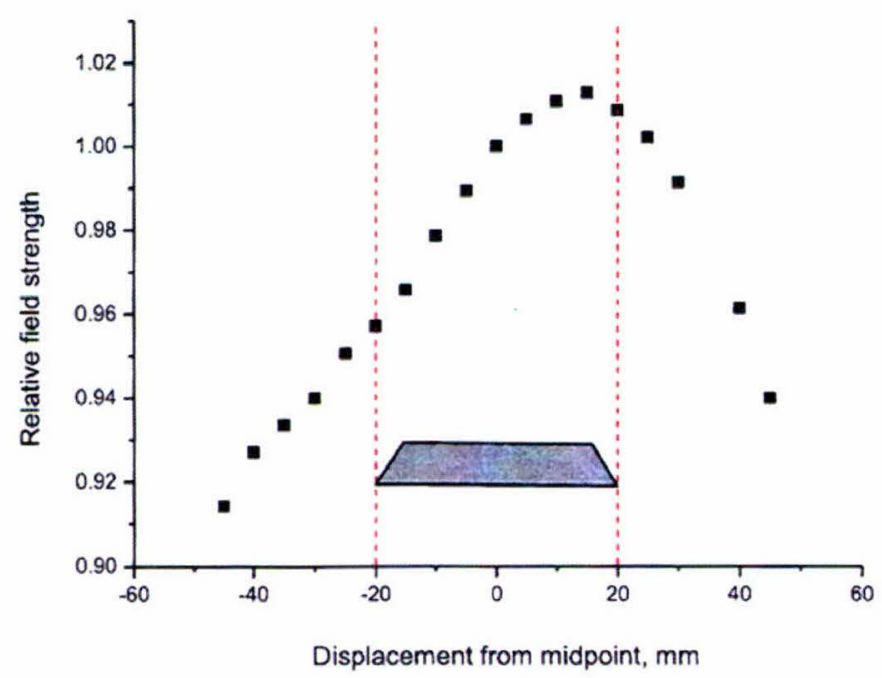

Figure 4.3: Positioning versus magnetic field strength for the Helmholtz coil. The dotted lines indicate the span of the sensor strip [5].

\subsubsection{Sensor Response as a Function of Magnetic Biasing Field}

The sensor response was now measured for different bias field strengths provided by the Helmholtz coil. This was done by measuring the absolute impedance of the reader coil over the range of $50 \mathrm{kHz}$ to $57 \mathrm{kHz}$ as observed on the impedance analyser. The relative signal strength was defined as:

$$
\frac{\Delta Z}{Z}=\frac{\left(Z_{r}-Z_{a}\right)}{1 / 2\left(Z_{r}+Z_{a}\right)} \times 100 \%
$$

where $Z_{r}$ is the resonant (maximum) impedance and $Z_{a}$ is the anti-resonant (minimum) impedance as shown in Fig. 2.21. The plot of Fig. 4.4 shows the results of sensor response as a function of magnetic biasing field for a Metglas $2826 \mathrm{MB}$ strip $40 \times 13$ $\mathrm{mm}$ in size.

The relationships were highly non-linear, and of expected shape as in [26]. The signal strength gradually increased to maximum where the bias field was equal to the anisotropy field $\mathrm{H}_{\mathrm{k}}$ of the sensor strip. At higher bias fields, the strip saturates, suppressing domain rotation and increasing eddy current losses, resulting in lowered signal strength. The change in resonant frequency is caused by the $\Delta \mathrm{E}$ effect as 
explained in Section 2.3.2.6, where the elasticity of the strip changes due to the applied magnetic field.

Maximum signal was found at $5.6 \mathrm{Oe}$, the same field which gave the lowest resonant frequency. This was then the optimum bias field required for maximum signal for $40 \times 13 \mathrm{~mm}$ sized Metglas strips.

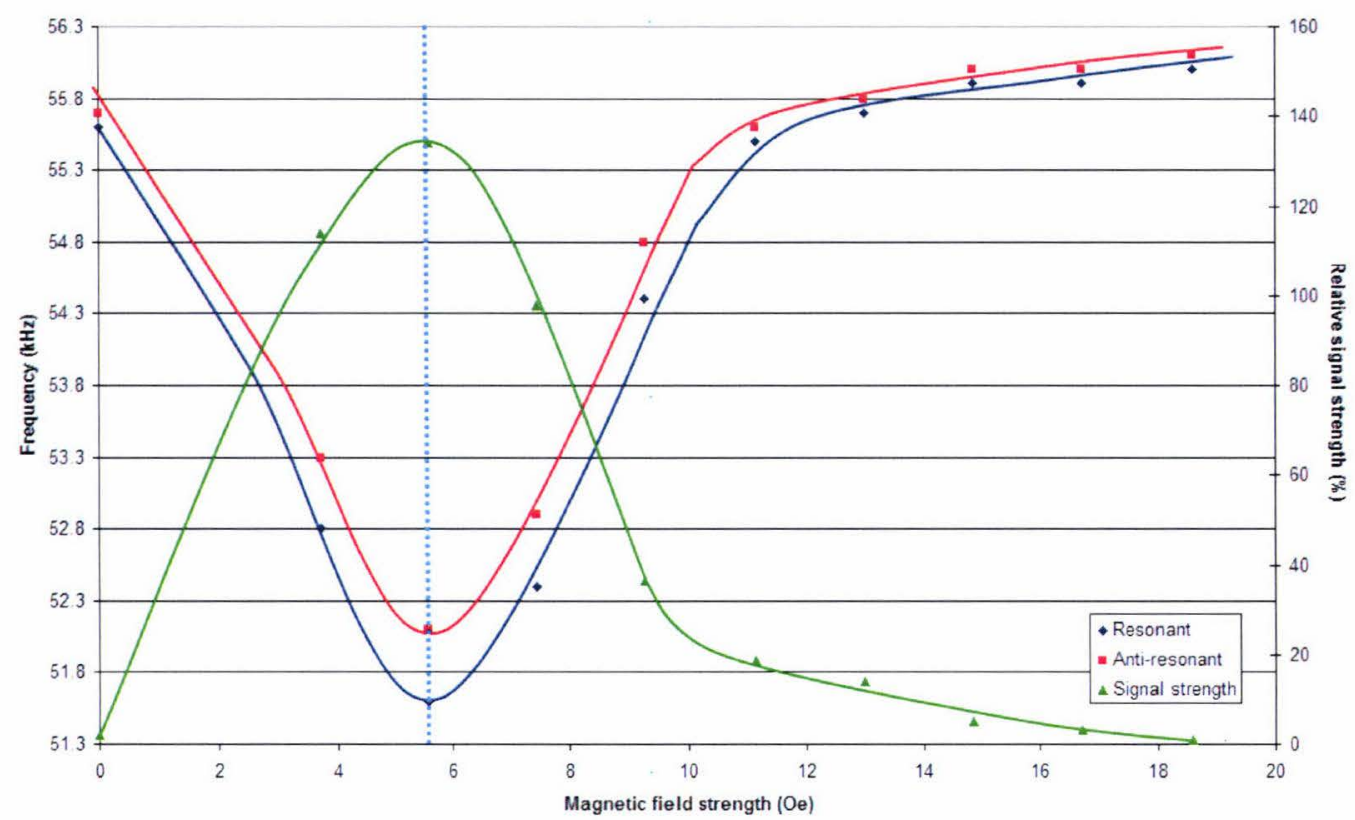

Figure 4.4: Biasing magnetic field strength versus resonant and anti-resonant frequencies as well as relative signal strength. The dotted line indicates the optimal field strength for the sensor strip.

\subsection{Biasing Magnet}

In practical sensor applications a Helmholtz coil cannot be used due to size and weight problems. In literature some systems have used DC coils for biasing purposes [2] [31]. However, it is thought that this could cause inaccuracies as it has been shown that the resonant frequency and signal strength is highly dependant on biasing field strength. This means that in order for the measurement to be reproducible, the biasing coil would have to be put in the exact same position every time. 
Because of these disadvantages, a magnetically hard metallic strip, attached to the sensor strip itself is a more convenient solution. Thus, a small metallic strip was magnetised and used as a biasing magnet by attaching it close to the sensor strip.

Since the biasing field strength versus frequency and signal size has been previously recorded by the use of the Helmholtz coil, the challenge was to replicate this by the use of the biasing magnet. The ideal magnetic strip would give the same sensor response as for the Helmholtz coil, as this would give predictable results.

It is explained in literature that a magnet can be attached to the centre of the sensor strip as at this point the vibration is minimal [50], and the damping effect is minimised. Therefore, the magnet was attached as shown in Fig. 4.5. The size of the steel strip was chosen to be the same as the sensor strips at $40 \times 13 \mathrm{~mm}$, as this would allow the sensor to be placed closer to the reader coil if the sensor is placed inside a container.

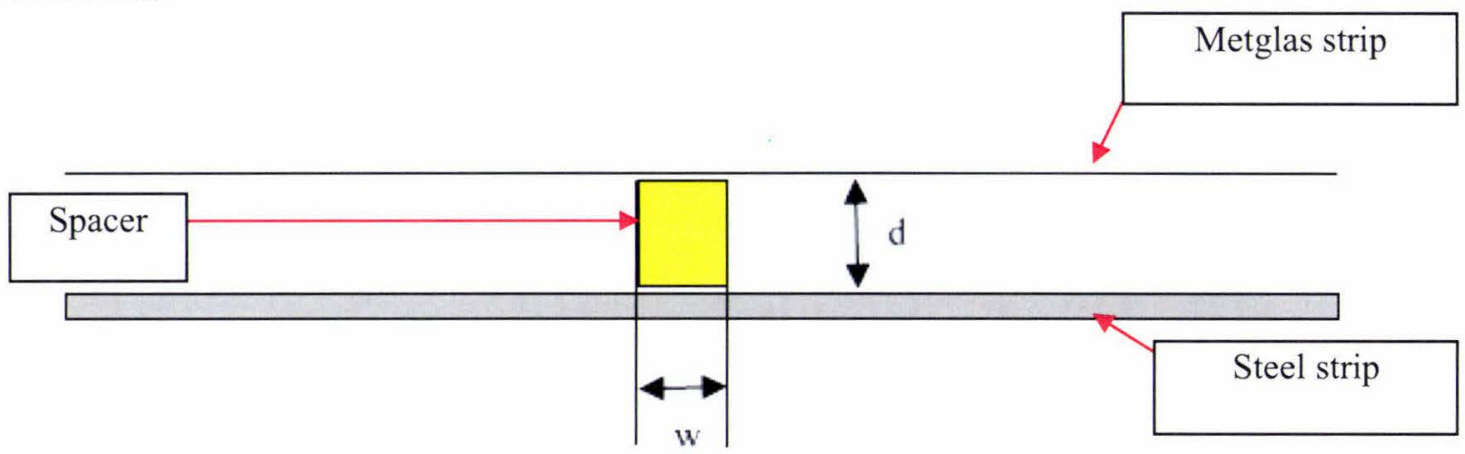

Figure 4.5: Side view of the sensor showing the Metglas sensor strip, spacer and biasing magnet. The distance between the magnet and sensor strip (d) and spacer width (w) are also indicated.

\subsubsection{Magnetising the Strip}

The biasing magnet was made from a sheet of spring steel, approximately $0.25 \mathrm{~mm}$ thick, which has been magnetised by placing it inside a DC coil, shown in Fig. 4.6. 


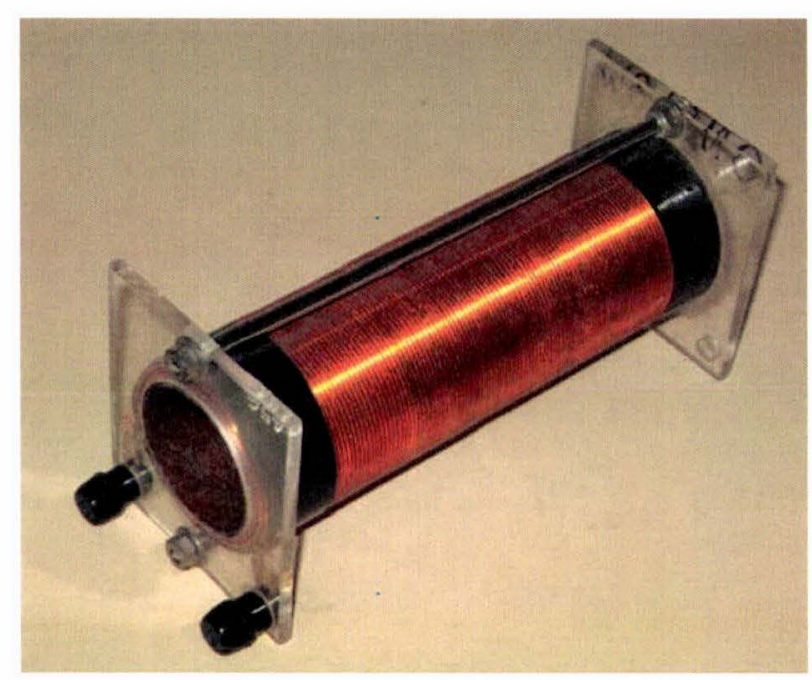

Figure 4.6: DC coil used to magnetise the spring steel strip.

The coil had the relationship between current and magnetic field, again measured with the gaussmeter (without the strip inside) as shown in Fig. 4.7. The relationship was linear with equation:

$$
H=37.184 I
$$

where $H$ is the magnetic field strength in Oe, and $I$ is the current in A. Since the magnetic fields were previously measured at the centre of the Metglas strip for the results using the Helmholtz coil, the magnetic field due to the steel strip was also measured at the centre of the strip with a gaussmeter.

The steel strip was found to reach saturation at applied field strengths of over 55 Oe, giving maximum field of approximately $13 \mathrm{Oe}$ at its central point. One point to note was that if the steel strip is bent or put under stress, it was found that its magnetisation would substantially drop. This means that once magnetised it must be handled with care otherwise it would need to be re-magnetised.

The steel strip was then magnetised to saturation and then left for a length of time, and was found that it could keep its magnetisation for a period in the order of weeks, indicating that it was suitable for use as a biasing magnet. 


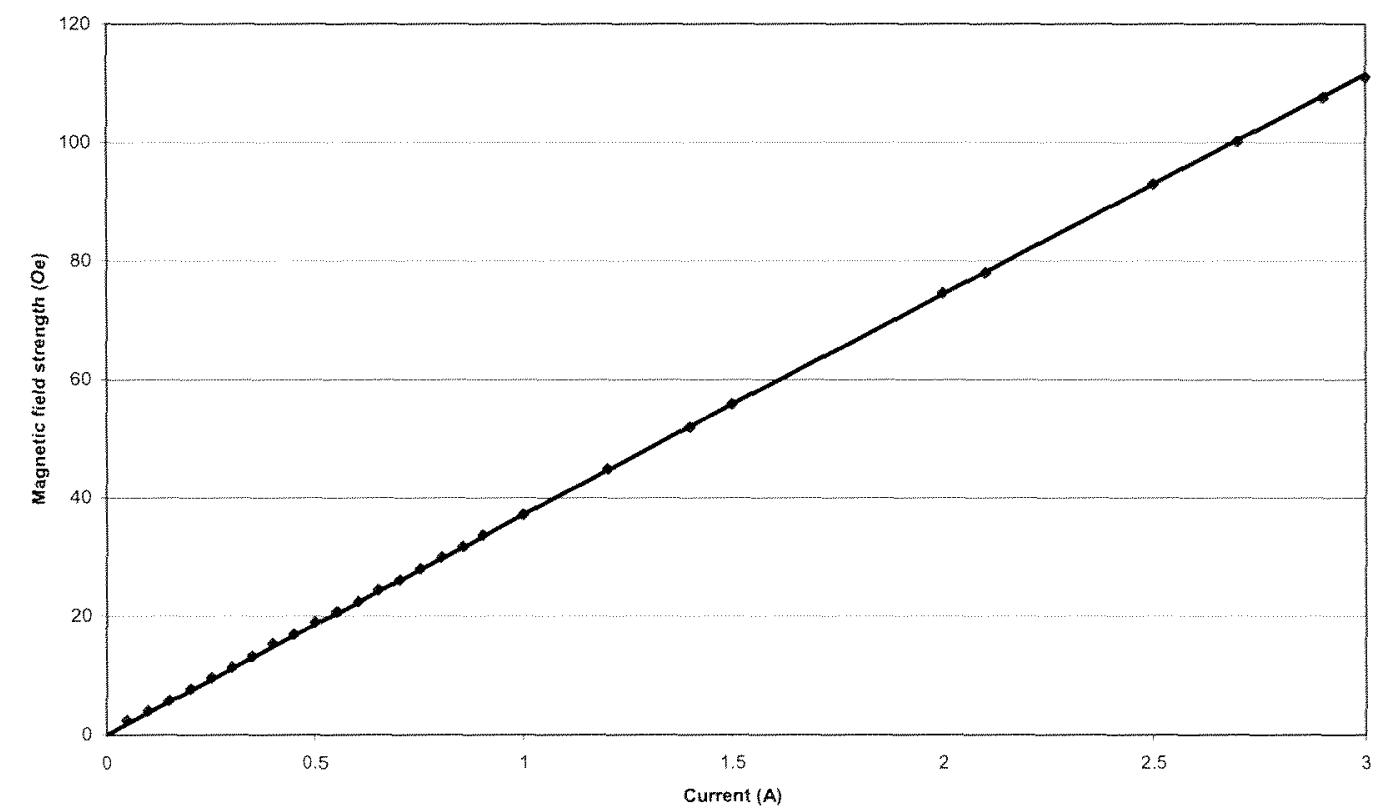

Figure 4.7: Coil current versus magnetic field strength for the magnetising coil.

\subsubsection{Distance from Strip}

The magnetic field from the steel strip was found to be strongest at the surface of the strip. The decrease in magnetic field strength with distance was investigated in order to attach the Metglas strip at a distance at which provides the optimal biasing field.

The $40 \mathrm{~mm}$ spring steel strip was magnetised to saturation, and its magnetic field at its centre point was measured at different distances with a gaussmeter, initially from the strip surface (zero distance), and the distance from the surface to gaussmeter probe was increased by taping layers of cardboard to the strip with double sided tape. The plot for distance versus magnetic field is shown in Fig. 4.8. The relationship was linear, and had the equation:

$$
H=-0.9502 d+13.645
$$

where $H$ is the magnetic field strength in Oe, and $d$ is the distance from the steel strip in $\mathrm{mm}$ as shown in Fig. 4.5. 


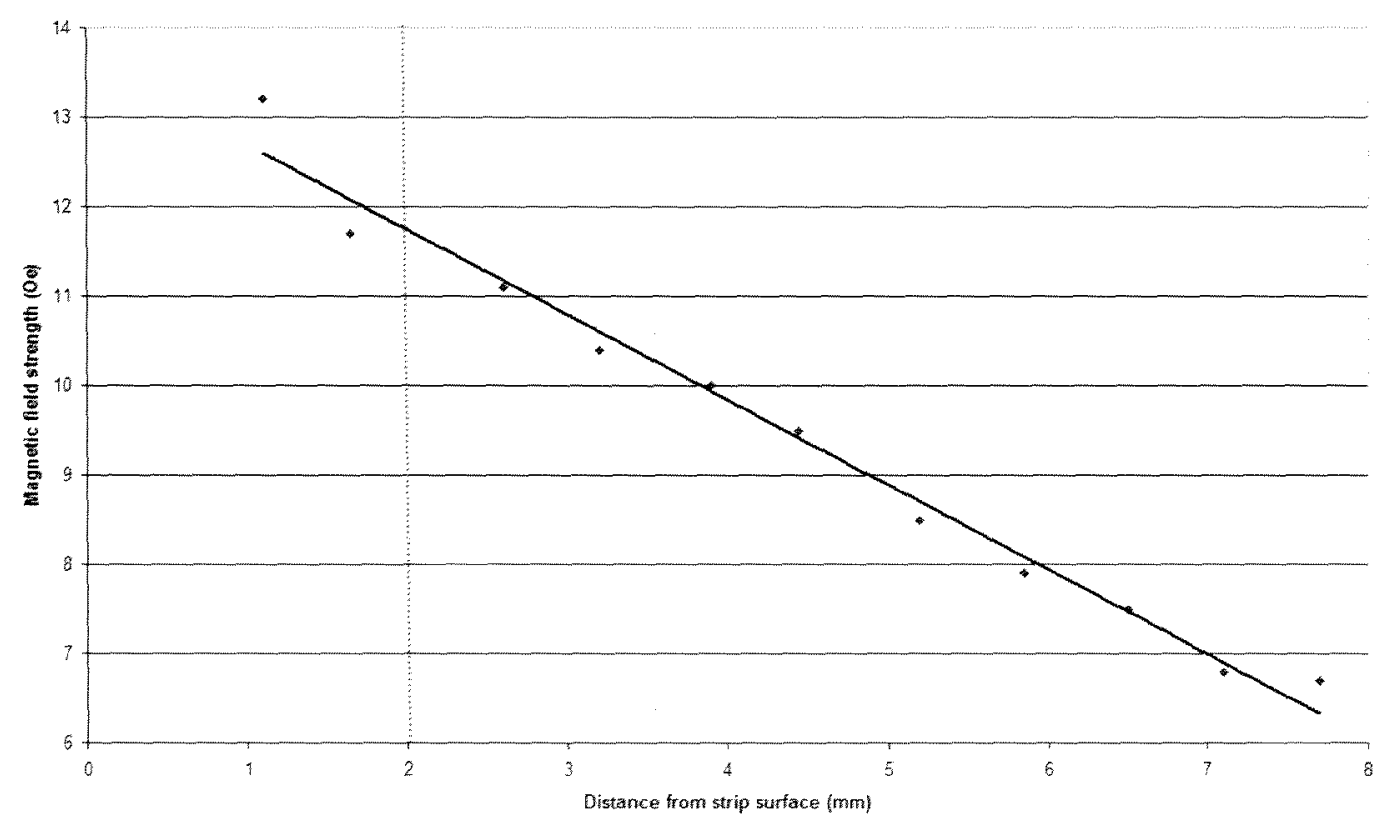

Figure 4.8: Distance from strip surface versus magnetic field strength. The red dotted line shows the lower limit to the relationship.

\subsubsection{Sensor Response with Biasing Magnet}

The $40 \mathrm{~mm}$ Metglas $2826 \mathrm{MB}$ sensor strip was placed at different distances from the magnet by placing a spacer of different heights between the magnet and Metglas strip as shown in Fig. 4.5. This was achieved by adding layers of small, thin plastic sheets as the spacer, and the width of the spacer was approximately $5 \mathrm{~mm}$. The three parts were put together with pieces of double sided tape.

During this experiment, a practical limitation on Eq. 4.4 was noticed, as it was found that the Metglas strip cannot reliably be placed closer than $2 \mathrm{~mm}$ to the magnet, as the strip was found to become attracted to the magnet and stick to it, causing damping. Therefore the practical maximum field strength achievable with $40 \mathrm{~mm}$ strips was just under 12 Oe. This is indicated on Fig. 4.8.

The distance from the steel strip to the Metglas strip was adjusted from $4 \mathrm{~mm}$ to $10 \mathrm{~mm}$, giving varying field strengths from approximately $9.5 \mathrm{Oe}$ to $4 \mathrm{Oe}$. The frequency response of this structure was compared to the Helmholtz results by placing it inside a reader coil, connected to an impedance analyser. The resulting plots are 
shown in Fig. 4.9 and 4.10 for resonant frequency and signal strength respectively. The plots show that the steel strip gives reasonably close results compared to the Helmholtz, although the Helmholtz tended to have the stronger signal strength, possibly due to more uniform field over the length of the strip. The resonant frequencies also tended to be slightly different with the steel strip giving higher frequencies than with the Helmholtz coil.

The final sensor design was to attach the biasing magnet $8.5 \mathrm{~mm}$ from the Metglas strip. According to Eq. 4.4, this distance gives the optimal biasing field of 5.6 Oe. The spacer used was a small piece of FR-4 (flame retardant 4) taken from a used printed circuit board giving a width of $3 \mathrm{~mm}$. The parts were stuck together with some cyanoacrylate based glue.

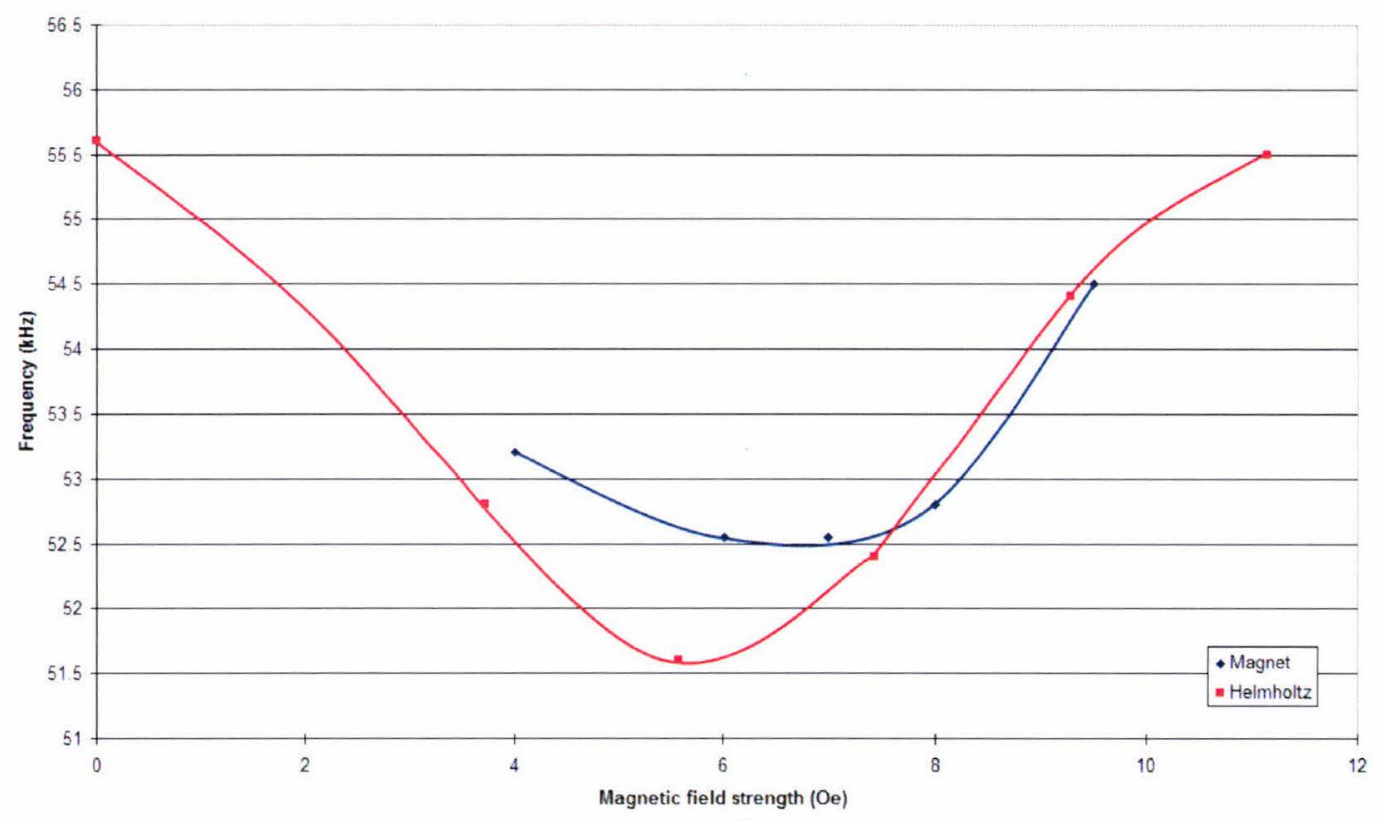

Figure 4.9: Biasing magnetic field strength versus resonant frequency with the Helmholtz coil and $40 \mathrm{~mm}$ bias magnet. 


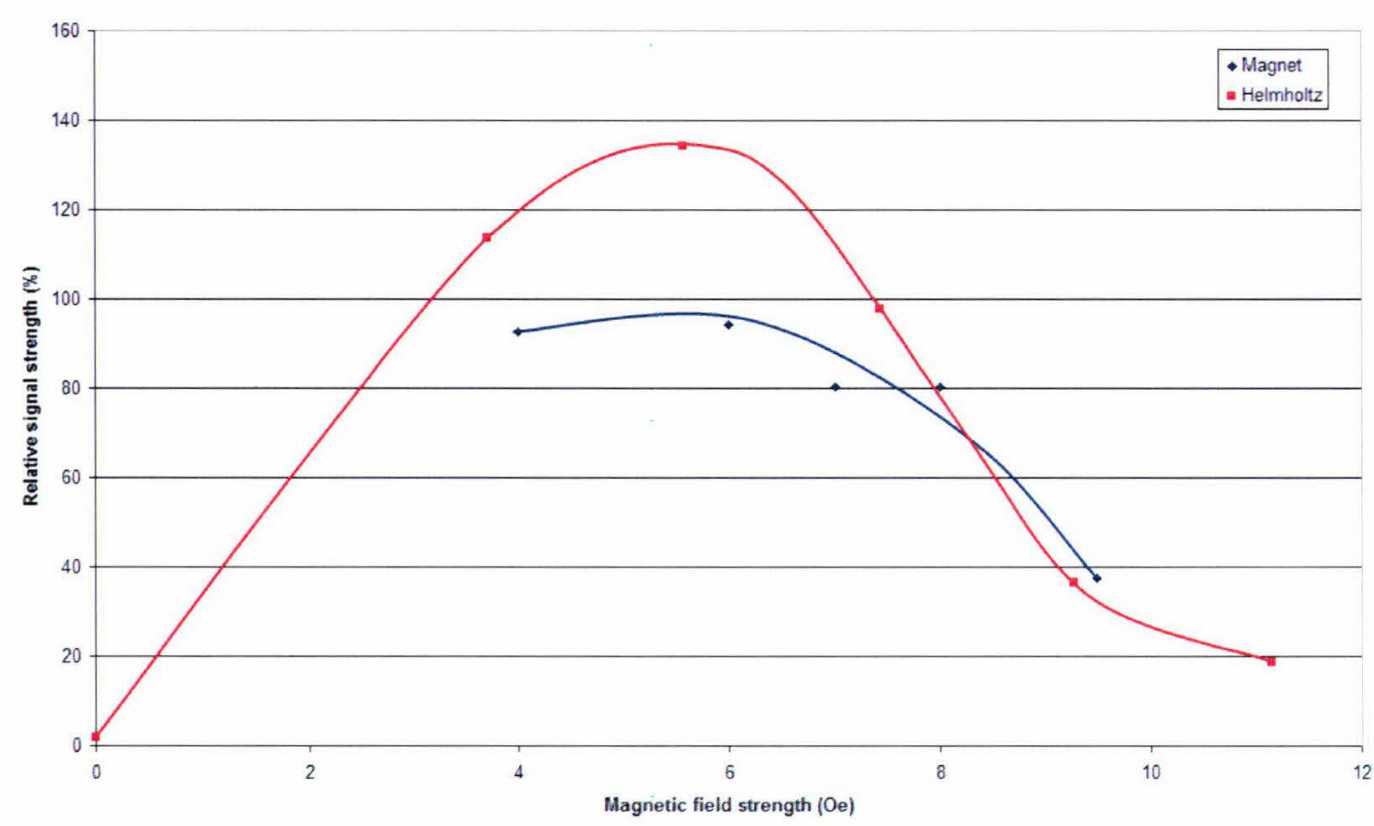

Figure 4.10: Biasing magnetic field strength versus relative signal strength with the Helmholtz coil and $40 \mathrm{~mm}$ bias magnet.

\subsection{Temperature Dependence}

It is essential to know the temperature sensitivity of a sensor element in order to separate out this contribution to the total response. A $40 \mathrm{~mm}$ Metglas strip was placed inside a reader coil, with a Helmholtz coil providing the biasing conditions. This arrangement was then placed inside a heated food container to give temperatures from room temperature $\left(\sim 18{ }^{\circ} \mathrm{C}\right)$ to about $60{ }^{\circ} \mathrm{C}$. The temperatures were measured with a thermocouple, and the container used is shown in Fig. 4.11.

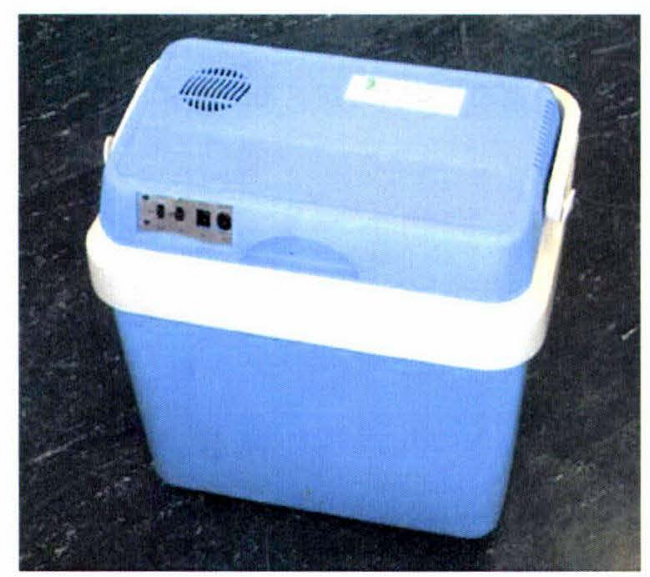

Figure 4.11: Heated food container used to measure temperature dependence. 
The reader coil was connected to the impedance analyser to measure the resonant frequency of the sensor strip by impedance de-tuning. The optimal biasing field of 5.6 Oe was investigated, as well as 3.7 Oe and 7.4 Oe to see the effect of different bias fields on either side of the optimal field. The resulting plot is shown in Fig. 4.12.

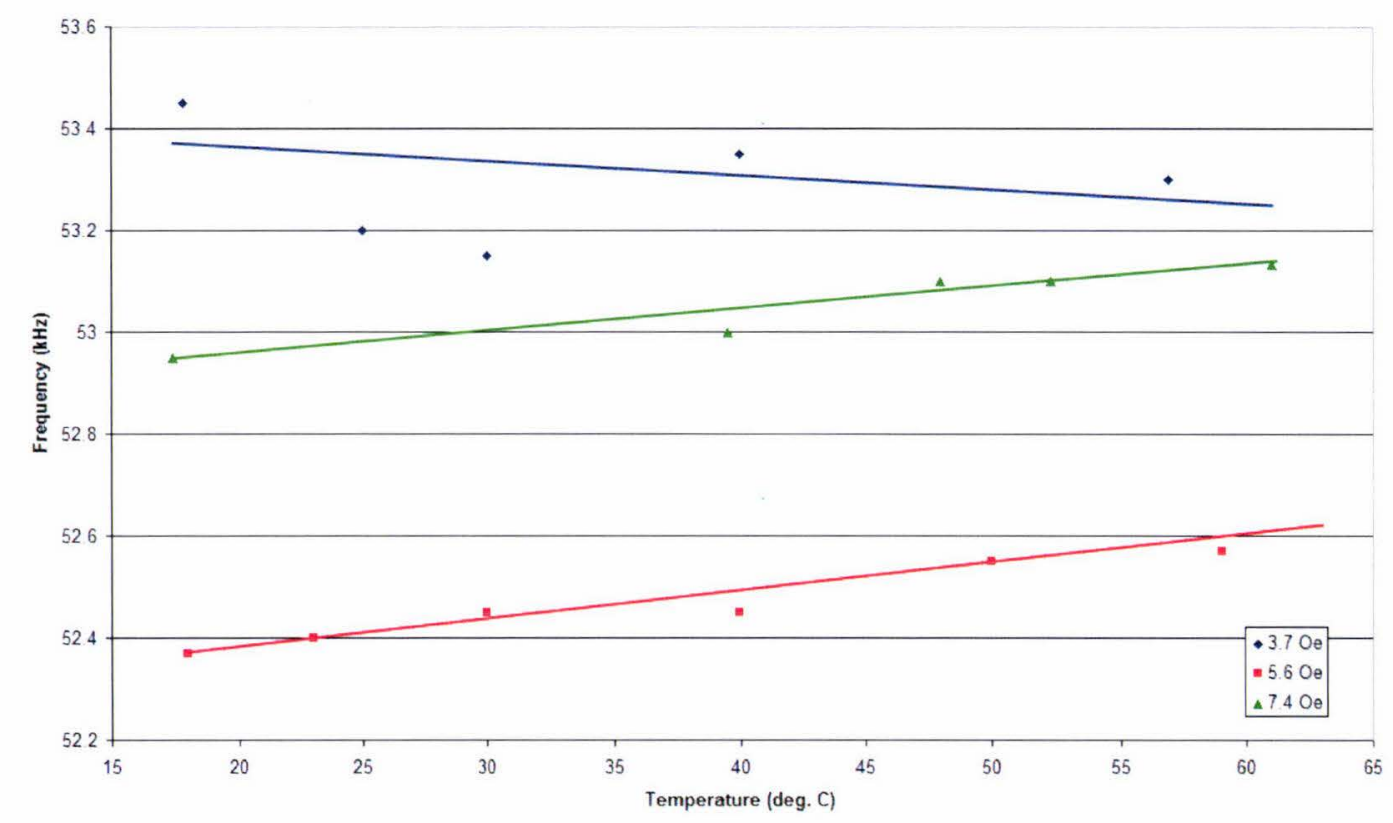

Figure 4.12: Temperature versus resonant frequency for three biasing fields.

It was found that in the field strengths tested, the resonant frequency did not change significantly with temperature. The sensitivity of the Metglas strip to temperatures was found to be $-0.7 \mathrm{~Hz} /{ }^{\circ} \mathrm{C}, 4.9 \mathrm{~Hz} /{ }^{\circ} \mathrm{C}$ and $4.5 \mathrm{~Hz} /{ }^{\circ} \mathrm{C}$ for $3.7 \mathrm{Oe}, 5.6 \mathrm{Oe}$ and $7.4 \mathrm{Oe}$ field strengths respectively.

The temperature dependence thus was found to be very weak in the tested biasing fields, and was similar to results obtained previously by Smith [5]. It also was previously shown in [5] that in order to achieve measurable temperature dependence one would require either a very high $(\sim 13 \mathrm{Oe})$ or very low $(\sim 1 \mathrm{Oe})$ biasing field, making a practical magnetoelastic temperature sensor difficult.

To observe the effect of temperature on a sensor strip with a biasing magnet attached, the prototype sensor constructed in Section 4.3 .3 was placed in the container. With 
this structure in the container, the resonant frequency was observed to decrease linearly with temperature, as shown in Fig. 4.13.

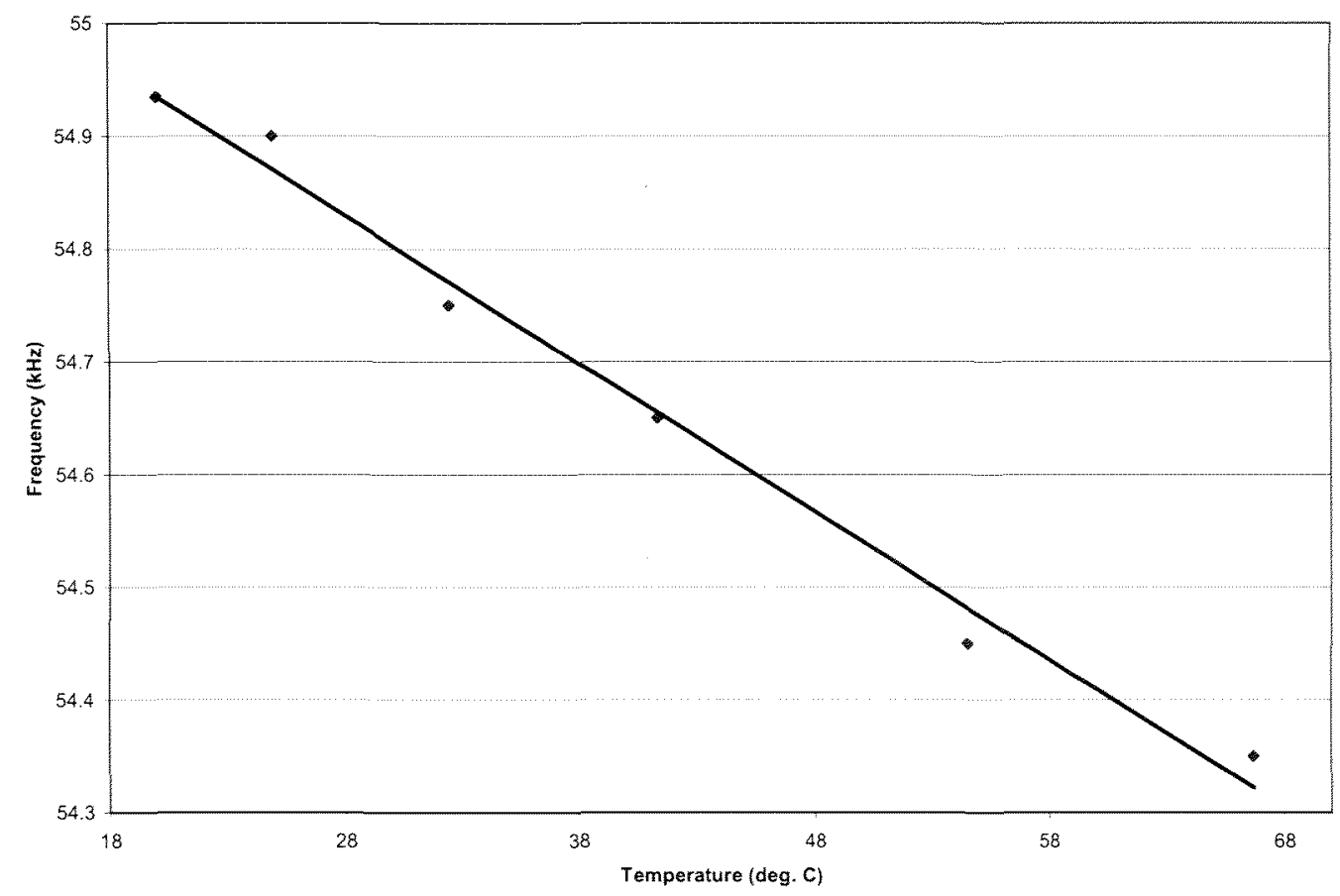

Figure 4.13: Temperature versus resonant frequency for a sensor with a biasing magnet.

The line of best fit was found to be:

$$
f=-0.0131 T+55.197
$$

where $T$ is the temperature in ${ }^{\circ} \mathrm{C}$, and $f$ is the resonant frequency in $\mathrm{kHz}$. This implies a sensitivity of approximately $-13 \mathrm{~Hz} /{ }^{\circ} \mathrm{C}$ in the temperature range of $20^{\circ} \mathrm{C}$ to $66.7^{\circ} \mathrm{C}$. This may have been due to thermal expansion of the spacer material, which was made from FR-4, the glue used to secure the parts, the steel biasing magnet, or even a combination of all three. Thermal expansion would have caused the biasing field to decrease, resulting in resonant frequency shift from the $\Delta \mathrm{E}$ effect.

Although the Metglas strip with the biasing magnet attached showed some temperature dependence, the change in frequency was caused by the spacer, glue or biasing magnet and not the sensor strip itself. Because of possible inconsistencies of this effect this was not investigated further as a possible temperature sensor. The 
temperature dependence of the sensor was found to have a maximum value of approximately $-13 \mathrm{~Hz} /{ }^{\circ} \mathrm{C}$ over the range of $20^{\circ} \mathrm{C}$ to $66.7^{\circ} \mathrm{C}$. If then the parameter of interest, such as humidity causes a large enough shift in resonant frequency of at least twice this value, then the temperature dependence can be neglected.

\subsection{Preparation of Humidity Sensor}

It was shown in Section 2.3.3.1 that an increase in sensor mass can lead to a drop in resonant frequency of the sensor. Thus, in order to create a humidity responsive sensor, we require a coating which absorbs the water molecules in the air, increasing the sensor mass. Ideally the coatings should be uniform over the strip for optimum performance, so each strip was visually inspected for uniformity after application.

It was also shown in Section 2.3.3.1 that previous work done [5] on humidity monitoring showed promising results with Metglas strips coated with polypyrrole (PPy) and polyvinyl alcohol (PVA). These solutions were again applied to the Metglas strips. The strips were $40 \times 13 \mathrm{~mm}$ in size as in previous sections, and they were initially cleaned in ethanol to remove contamination and then dried before coatings were applied.

The coatings were all applied on the rough side of the strip to improve adhesion, as being more friction it would make it harder for the coatings to come off. There are some other practical advantages to this method, one being that if the centrifuge (see Section 4.5.1) is used to coat the strip, one side must always be attached to the rotating stage. If both sides are to be coated, one coated side must be attached to the stage to coat the other side, and when removing from the stage the coating can be damaged. Another advantage is that the coating can be made more consistent if only one side is done.

\subsubsection{Centrifuge}

A centrifuge, shown Fig. 4.14, normally used to mix samples by spinning, was used as a spin coater. The test tube holders were removed, and the sample was attached to 
the central rotating stage with a piece of double sided tape. In the centre of the cover of the centrifuge is a small hole, and this is where the solutions were added.

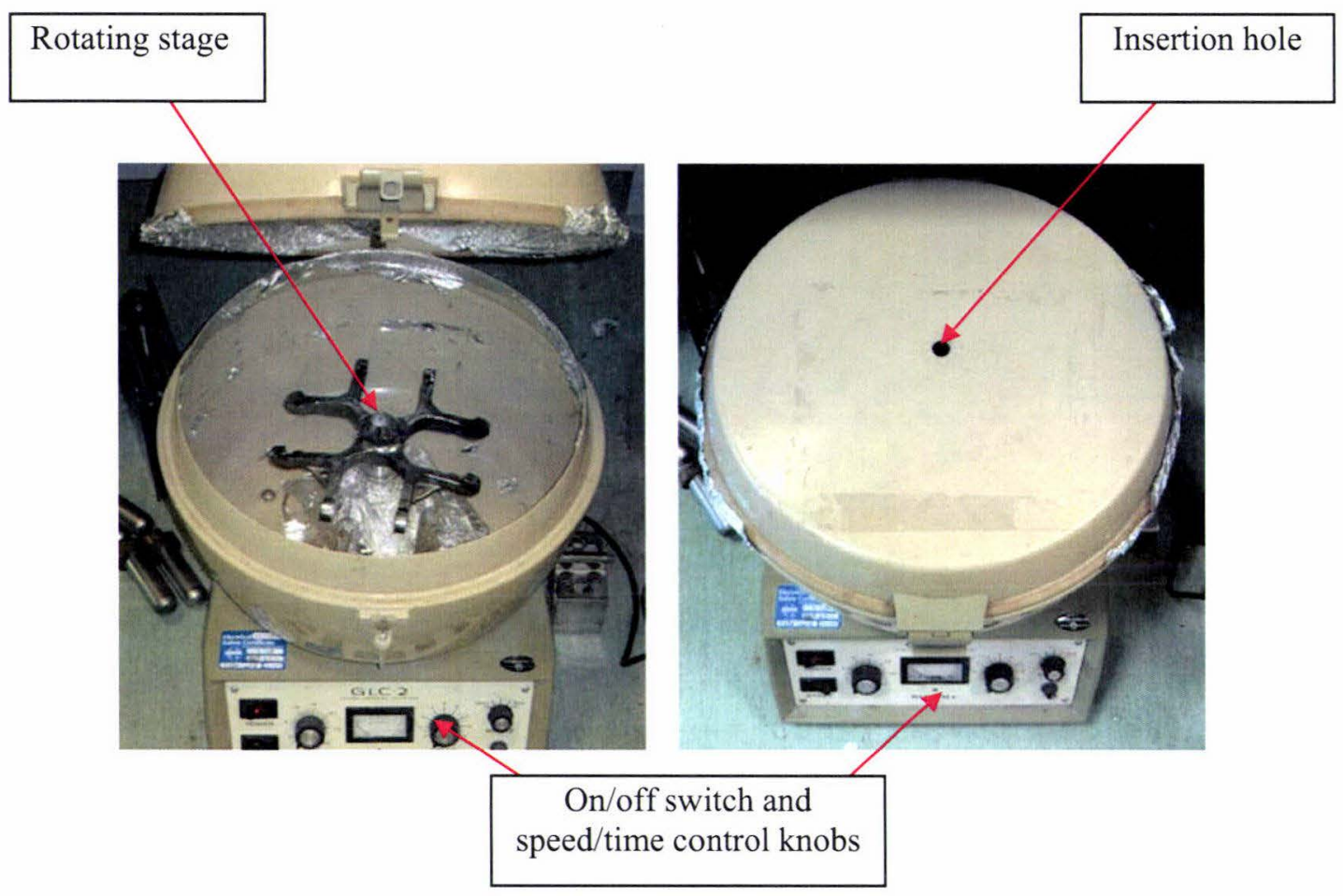

Figure 4.14: The centrifuge used as a spin coater with cover open (left), and closed (right).

\subsubsection{Polypyrrole (PPy)}

Polypyrrole is a black coloured, low viscosity solution. It is a conducting polymer which is used in batteries, corrosion protection applications and in actuators [51]. Its chemical structure is shown in Fig. 4.15.

An important point found is that the solution must initially be shaken in its bottle. Neglecting this causes the coating to become very powdery and can wipe off easily. The initial application method was to dip-coat, but this was found to be difficult in achieving an even coating, as the solution was of low viscosity and would flow off easily from the Metglas strip. Spin coating was also attempted but the solution splattered inside the spin coater due to its low viscosity and it was found that none is left on the strip after opening the cover. 
The method found to work well was to put the Metglas strip flat on a piece of sponge (rough side up), and slowly flow the solution onto the strip. Initially the Metglas, being hydrophobic repels the solution, but over time it is able to make contact with the strip. Excess solution on the strip is absorbed by the sponge. The strip is then allowed to dry on the sponge for about 30 minutes. Most of the surface could be covered but some of the edges were still uncovered so a second layer was added. The resulting coated strip is shown in Fig. 4.15.
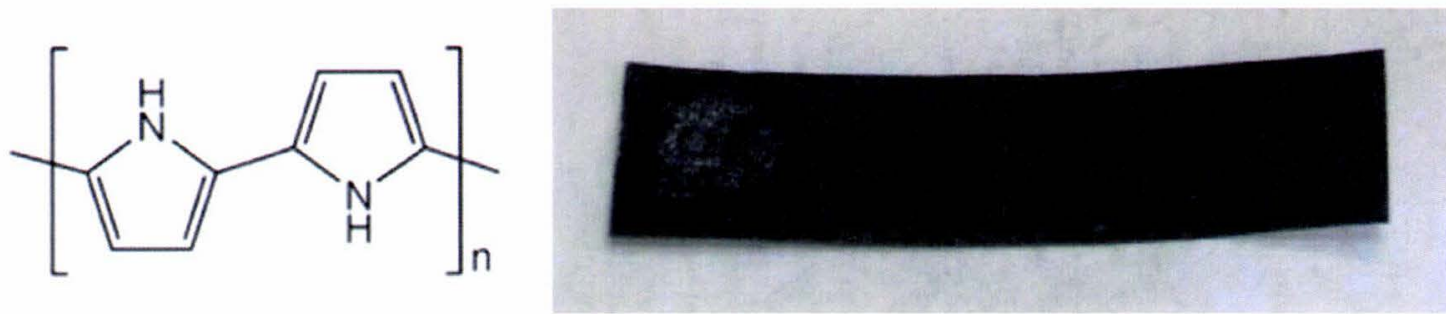

Figure 4.15: Chemical structure of PPy (left) and Metglas strip coated with PPy (right).

Since the shiny uncoated side of the strip is in contact with the sponge, it can leave some unintended patches of PPy. This was removed by wiping in a circular motion with cotton buds dipped in toluene. The final sensor strip had a mass of $0.1105 \mathrm{~g}$, up from $0.1077 \mathrm{~g}$ uncoated.

\subsubsection{Polyvinyl Alcohol (PVA)}

This is a moderately viscous, non-toxic, odourless white to clear liquid, commonly used as a thickener, emulsifier or binder in applications such as glues [51], as well as a moisture barrier for supplement tablets. Its chemical structure is shown in Fig. 4.16.

The PVA solution was applied with the spin coater. A burette was not used as this made the addition of the solution too slow, due to its viscosity. Instead, a syringe was used. It was found that a rotation speed of 3000 RPM tended to create a relatively large "hole" in the coating at the centre of the strip, so 2000 RPM was used. The strip was attached to the rotating stage with double sided tape (rough side up), and spun at 2000 RPM. $5 \mathrm{~mL}$ of PVA was slowly added to the spinning strip with a syringe. The strip was spun for a further 5 minutes to ensure the coating was dry. The resulting coated strip is shown in Fig. 4.16. 

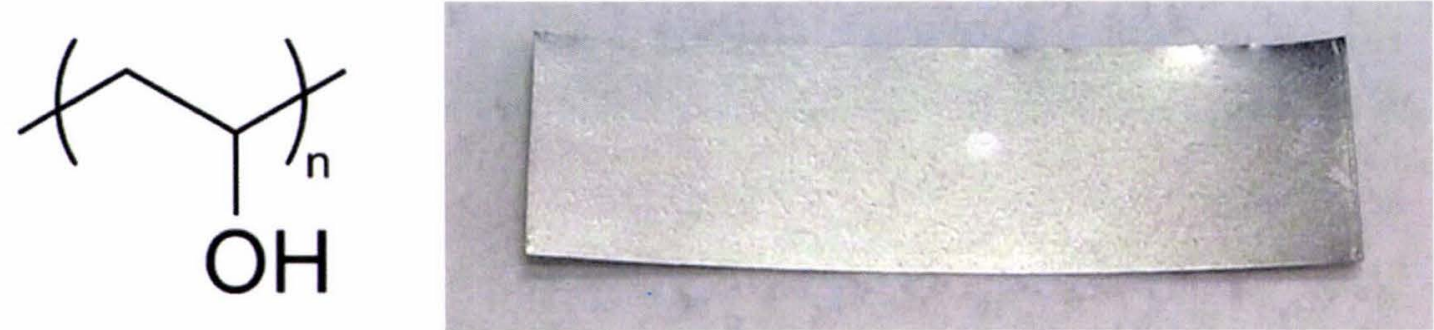

Figure 4.16: Chemical structure of PVA (left) and Metglas strip coated with PVA (right).

The coating still had a small "hole" at the centre so another layer, using the same method, was applied. The final sensor strip had a mass of $0.1070 \mathrm{~g}$, up from $0.1030 \mathrm{~g}$ uncoated.

\subsection{Experimental Arrangement and Procedure for Humidity Tests}

Humidity tests were performed using an existing gas flow control system constructed by Holt [52] and Zhen [53], shown in Fig. 4.17. This system is constructed from $6 \mathrm{~mm}$ stainless steel tubing and contains a series of mass flow controllers and air operated valves, which can accurately control the path and flow volume of gas through the system. Instrument grade air is used as a carrier gas and the initial flow is split into different paths at an inlet manifold. One of these paths allows the carrier gas to flow through a liquid source, called a bubbler, resulting in the vapour of this source material being carried into the test cell (shown Fig. 4.18).
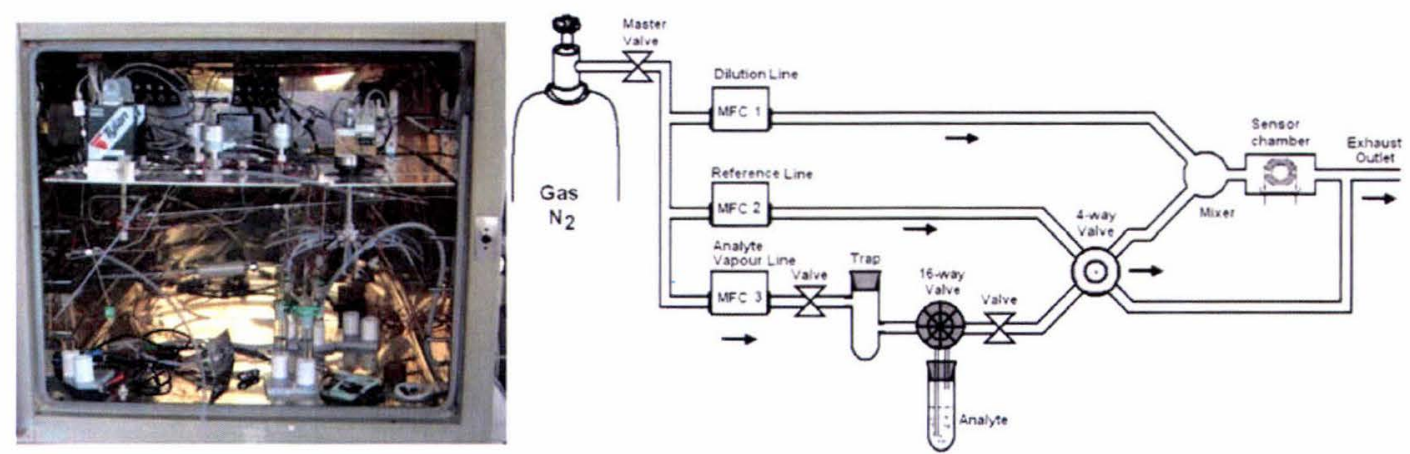

Figure 4.17: The gas flow system constructed by Holt and Zhen.

It has been shown in [53] that the system allows the carrier gas stream to completely saturate with the vapour source. Just before the vapour enters the test cell, it can be diluted by means of a dilution stream. The entire system is controlled by a PC using a 
LabVIEW [54] software interface. In these experiments the bubbler was filled with water and a total flow rate of $200 \mathrm{cc} / \mathrm{min}$ into the test cell was maintained. This flow volume was controlled in different ratios between the bubbler and the dilution line.

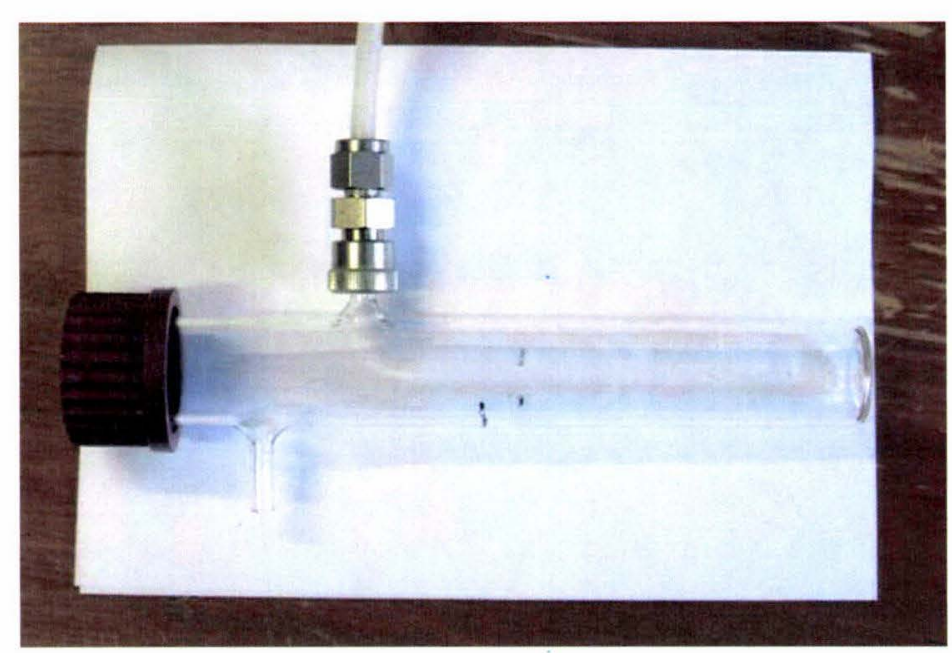

Figure 4.18: The glass test cell showing the inlet valve and tube, removable cap and exhaust port.

The relative humidity $(R H)$ of water vapour is the ratio of actual vapour density to the saturation density at that temperature [55]:

$$
R H(\%)=\frac{\rho_{\text {actual }}}{\rho_{\text {saturation }}} \times 100 \%
$$

where $\rho_{\text {actual }}$ is the actual vapour density and $\rho_{\text {saturation }}$ is the saturation vapour density. If we assume that the air stream flowing through the bubbler becomes completely saturated with water vapour, we can express the expected relative humidity in terms of the flow rate through the bubbler and dilution line as:

$$
R H(\%)=\frac{f_{\text {vapour }}}{f_{\text {vapour }}+f_{\text {dilution }}} \times 100 \%
$$

where $f_{\text {vapour }}$ is the flow rate of the water vapour from the bubbler, and $f_{\text {dilution }}$ is the flow rate of the dilution line. It has previously been shown by Zen [53] that this 
saturation condition is satisfied for all but very high flow rates in the sensor test system.

The relative humidity was also measured by means of a commercial humidity sensor, the Honeywell HIH-4000 3-terminal IC humidity sensor (cost approx. \$56NZ) shown in Fig. 4.19 [56]. This sensor contains a polymer capacitive element with on-chip signal conditioning, and is specified as having an accuracy of $\pm 3.5 \%$ and a stability of $\pm 0.2 \%$ at $50 \%$ relative humidity per year. The humidity sensing range is specified from $0 \%$ to $100 \%$, and the response time is specified as 15 seconds in slow moving air. The calibration sheet for the IC sensor, provided by the manufacturer specified the following output voltage with relative humidity, at $25^{\circ} \mathrm{C}$ :

$$
R H(\%)=\frac{\left(0.98 V_{\text {out }}-0.8049\right)}{0.03055}
$$

where $R H$ is the relative humidity and $V_{o u t}$ is the output voltage in $\mathrm{V}$. This IC sensor was connected to a National Instruments data acquisition board and PCI card on PC, which measures the output voltage from the IC sensor. Note that the 0.98 factor is present because the supply voltage from the data acquisition board was not $5 \mathrm{~V}$ but actually $4.9 \mathrm{~V}$, and so it acts as a correction factor. This was recommended in the calibration sheet for the sensor.

A simple LabVIEW program to convert the output voltage from the IC sensor to a relative humidity reading was written to help make measurements easier. Three wires were soldered to the leads of the IC sensor and are colour coded; red is $+5 \mathrm{~V}$, black is ground, and blue is the output voltage. The front panel for the LabVIEW program and IC sensor is shown in Fig. 4.19. 

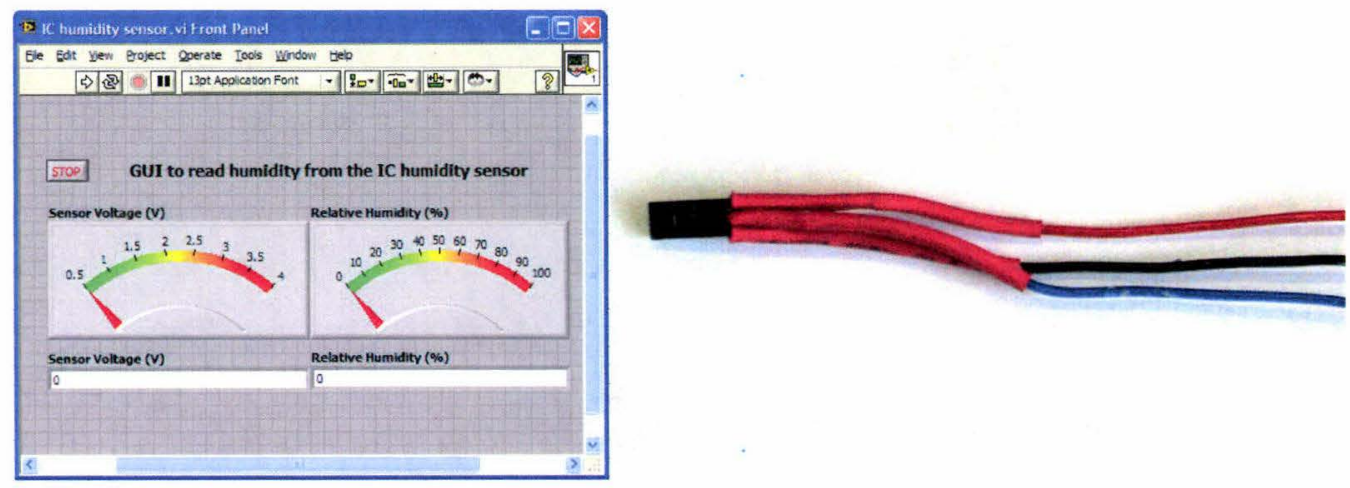

Figure 4.19: LabView user interface to read the relative humidity (left) from the Honeywell HIH-4000 IC humidity sensor (right).

In Fig. 4.20 the expected relative humidity values as calculated from the flow rates are compared to the corresponding relative humidity values as measured by the HIH- 4000 . These values show good agreement, with marginally lower values being indicated by the sensor. This is most likely due to non-saturation at the higher flow rates, or the condensation on any colder parts of the system that will lower the vapour pressure.

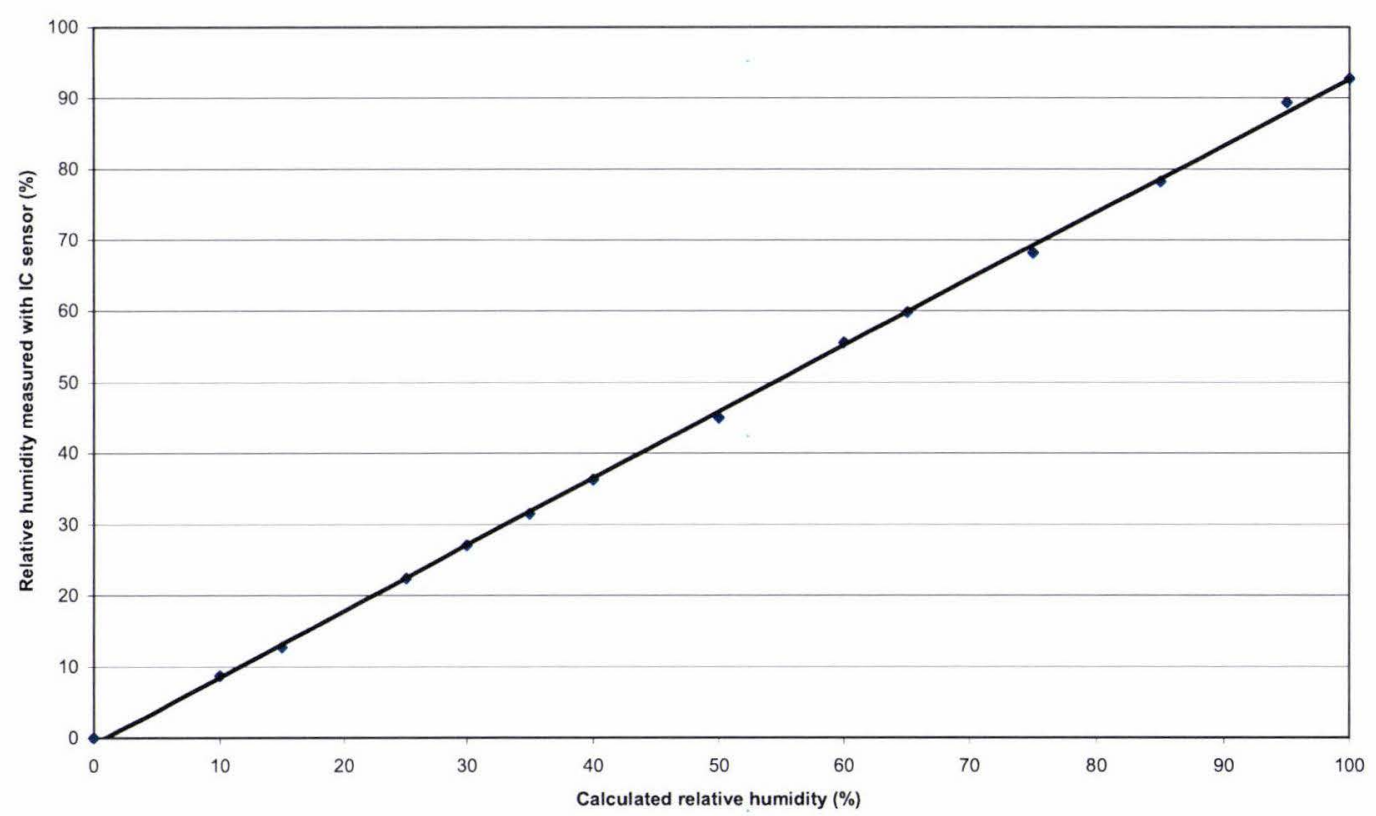

Figure 4.20: Calculated relative humidity versus values measured by the IC sensor.

Initial tests on the coated Metglas strips were performed using the system as described in Section 4.2. The experimental setup is shown in Fig. 4.21. The only difference to the original arrangement was the addition of the commercial Honeywell humidity 
sensor to the test cell. This was incorporated into the test cell using a simple feedthrough system for the sensor pins.

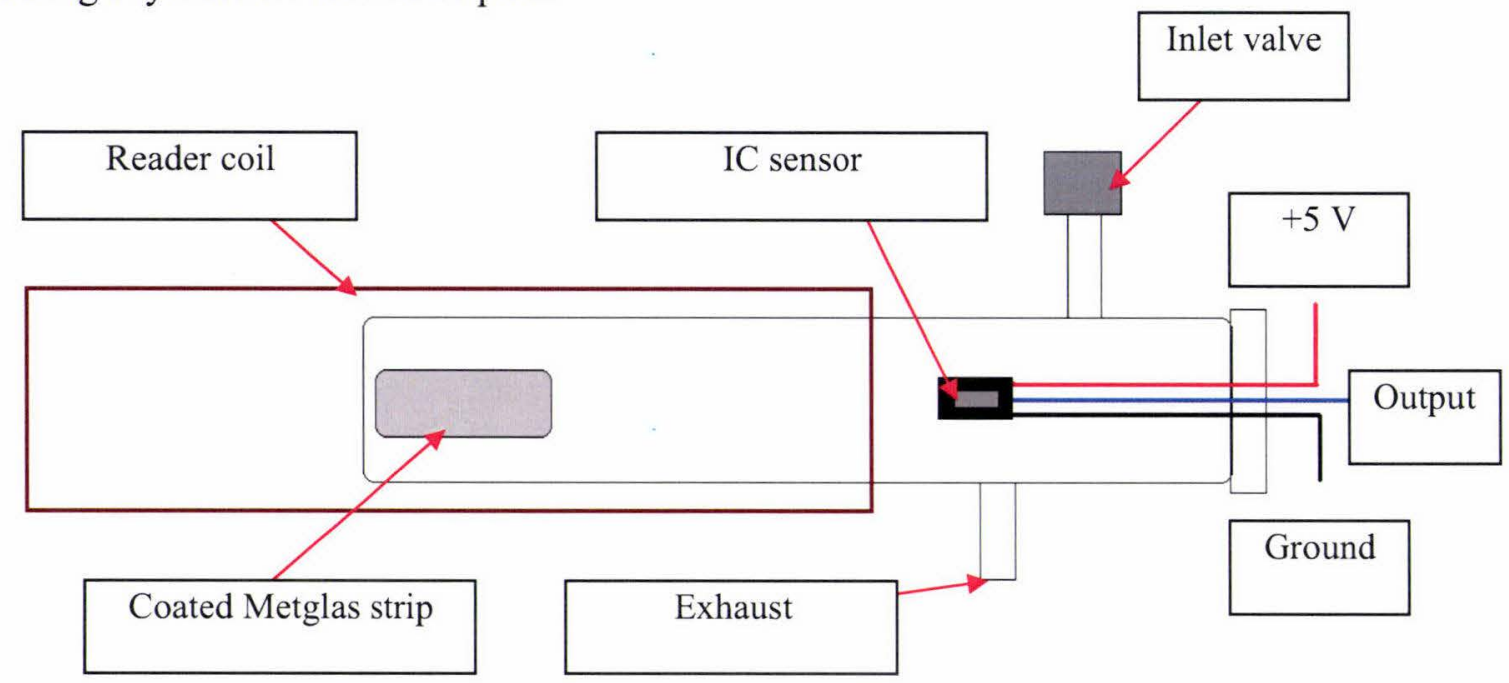

Figure 4.21: Experimental arrangement for humidity testing.

\subsection{Initial Evaluation of Humidity Sensor Response}

Initial tests were performed using standard impedance de-tuning methods. The biasing field was first varied, and was found that for all cases the field strength which gave the strongest signal was the same as for uncoated sensors - 5.6 Oe, and thus results shown are all values obtained using this biasing field. The relative humidity inside the test cell was initially lowered to near $0 \%$, and then this was increased gradually to about $90 \%$.

\subsubsection{Response of Uncoated Sensor strip}

In order to establish a baseline for the response of magnetoelastic humidity sensors, the response of an uncoated Metglas strip was first measured. The resonant frequency of the sensor strip is plotted against relative humidity, measured by the IC sensor in Fig. 4.22.

It can be seen that the uncoated strip does not appear to show any response to the variation in the humidity in the humidity levels between $0 \%$ and $60 \%$ relative humidity. Over this range the sensor resonant frequency remains constant at 
$\sim 53.35 \mathrm{kHz}$. As the humidity is increased further, we find a decrease in the resonant frequency, with an approximate rate of $3 \mathrm{~Hz} / \mathrm{RH}(\%)$.

A similar result was also obtained by Smith [5]. One possible explanation for this sensor response may be due to some condensation taking place on the strip. This will lead to a mass loading effect and thus a decrease in resonant frequency. The condensation could have been caused by a difference in temperature between the bubbler and the test cell. If the bubbler was of higher temperature, then at higher humidity levels we may find condensation taking place inside the test cell causing a decrease in resonant frequency.

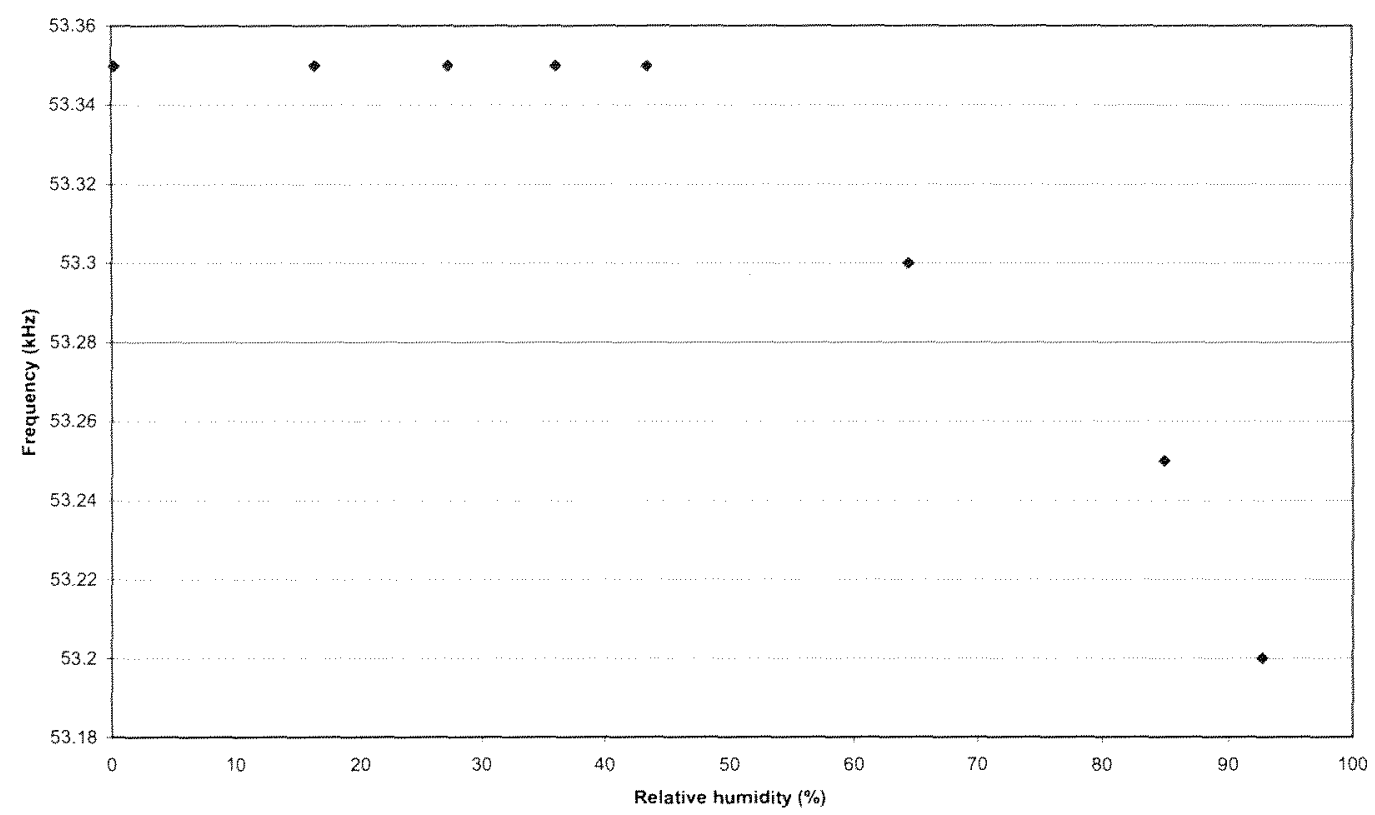

Figure 4.22: Relative humidity versus resonant frequency for an uncoated sensor.

\subsubsection{Polypyrrole (PPy)}

The Metglas strip coated with PPy as discussed in Section 4.5.2 was placed in the test cell. The plot of relative humidity versus resonant frequency is shown in Fig. 4.23. The sensor strips took about 30 minutes to respond fully to changes in humidity. The sensor's resonant frequency shifted from $51.45 \mathrm{kHz}$ to $50.90 \mathrm{kHz}$ for a change in humidity from $5.7 \%$ to $47.86 \%$ humidity respectively. However, as Fig. 4.23 shows, 
the response began to saturate at a humidity of about $35 \%$, making measurement of higher humidity levels above $50 \%$ not possible.

In Section 2.2.3.1 it was shown that in previous studies of PPy the resonant frequency shift was linear up to $80 \%$ humidity. In this occasion, the response was only linear up to about $30 \%$ relative humidity. This may have been due to the fact that the solution was applied differently.

The line of best fit for the relationship between resonant frequency and relative humidity for humidity levels within the non-saturating $(0-30 \%$ humidity) region was:

$$
f=-0.0224 R H+51.577
$$

where $f$ is the resonant frequency in $\mathrm{kHz}$ and $R H$ is the relative humidity in $\%$, thus the sensitivity of this sensor is $22.4 \mathrm{~Hz} / \mathrm{RH}_{\%}$. A possible improvement is to give more coats to the sensor strip, resulting in perhaps a larger humidity range it can measure.

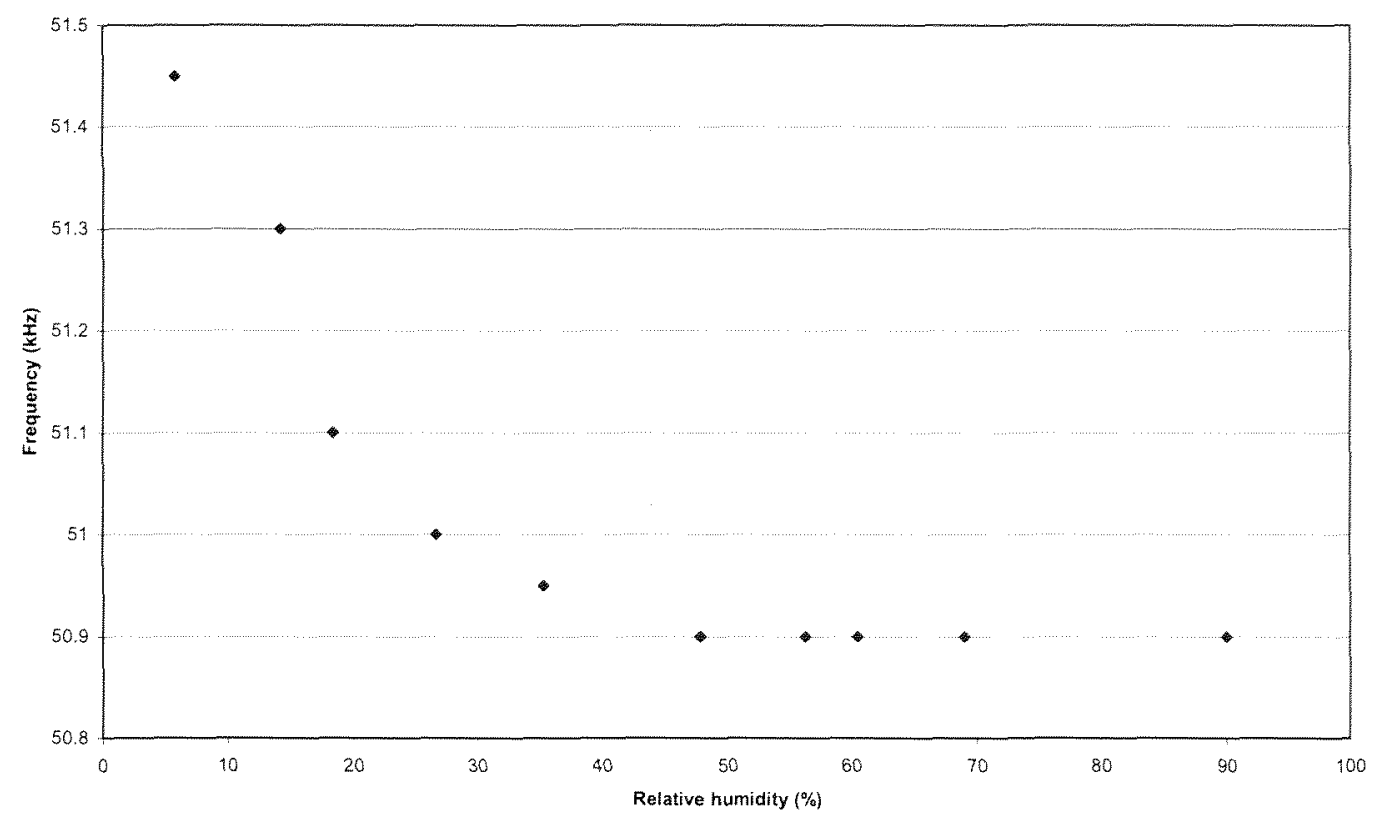

Figure 4.23: Relative humidity versus resonant frequency for the PPy coated sensor. 


\subsubsection{Polyvinyl Alcohol (PVA)}

The PVA coated Metglas strip as discussed in Section 4.5 .3 was now placed in the test cell. The sensor strip took about an hour until the resonant frequency stabilised. The resulting plot of Fig. 4.24 shows the relative humidity as measured by the IC sensor versus resonant frequency. The strips coated with PVA were found to be much more responsive to the changes in humidity compared to PPy, with the frequency ranging from $54.95 \mathrm{kHz}$ to $51.85 \mathrm{kHz}$ in humidity levels between $5.7 \%$ and $69 \%$ respectively. When the humidity levels were increased to over $70 \%$ however, the graph shows that the sensor response starts to saturate, giving smaller changes in frequency.

Interestingly, the response compared to previous results shown in Section 2.3.3.1 was substantially different, where previously the response was more non-linear. Again this may have been due to different coating methods used.

The line of best fit for the relationship between resonant frequency and relative humidity for humidity levels within the non-saturating $(0-70 \%$ humidity) region was:

$$
f=-0.05 R H+55.146
$$

Where $f$ is the resonant frequency in $\mathrm{kHz}$ and $R H$ is the relative humidity in $\%$. Thus the sensitivity of this sensor is approximately $50 \mathrm{~Hz} / \mathrm{RH} \%$. 


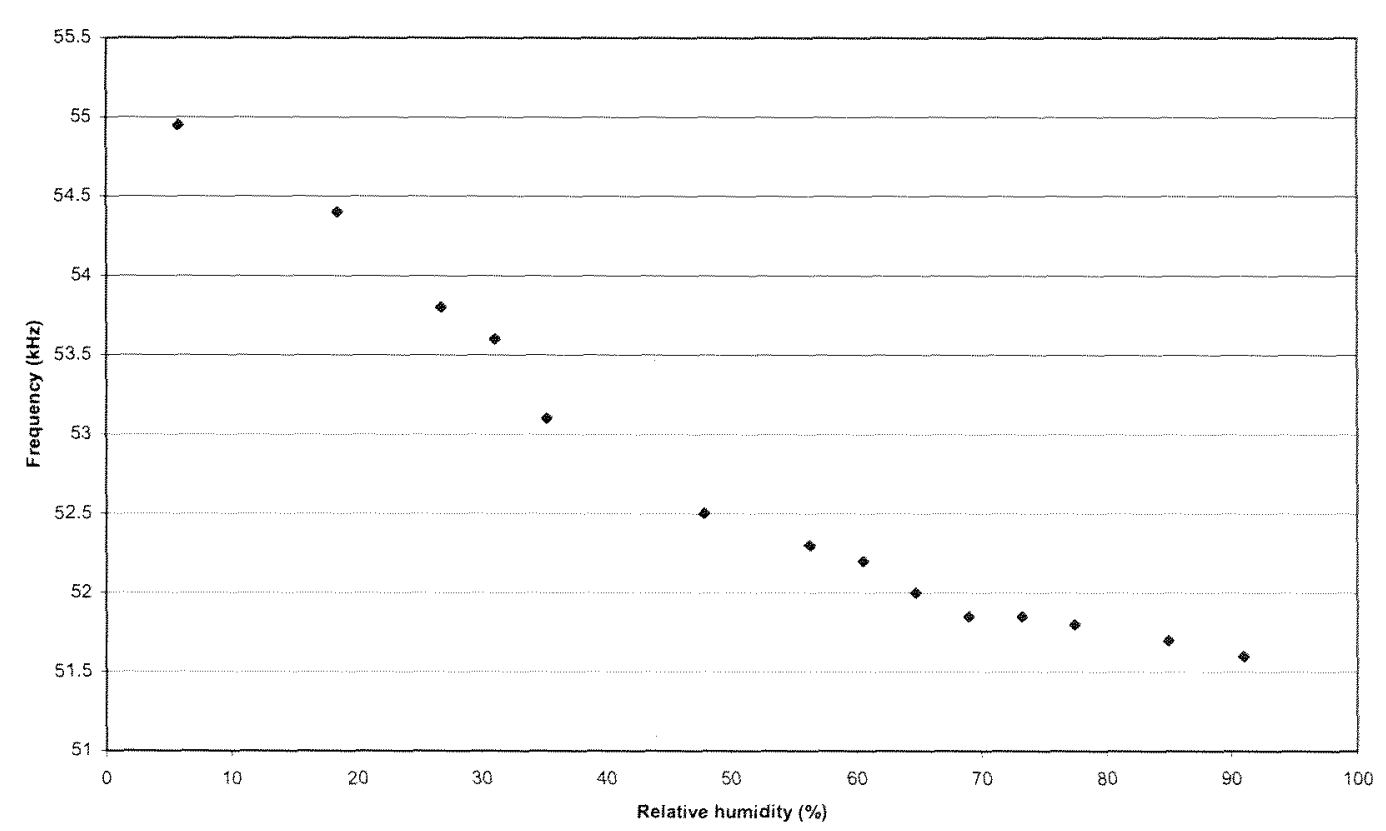

Figure 4.24: Relative humidity versus resonant frequency for the PVA coated sensor.

\subsubsection{Discussion}

Comparing the results of the PPy and PVA coated sensors, the PVA coated sensor was found to have the superior response, giving a larger frequency shift per change in relative humidity $(22.4 \mathrm{~Hz} / \mathrm{RH} \%$ vs. $50 \mathrm{~Hz} / \mathrm{RH} \%)$, as well as having a wider responsive humidity range. The sensitivity of $50 \mathrm{~Hz} / \mathrm{RH} \%$ also easily compensates for the temperature dependence of $-13 \mathrm{~Hz} /{ }^{\circ} \mathrm{C}$ as discussed in Section 4.4. The only disadvantage was that the PVA took longer to respond to changes in humidity (30 minutes versus 1 hour). The fact that the PVA solution can be spin coated is another advantage because it should give more repeatable results.

Both sensors were found to give the largest signal when the DC magnetic field was about 5.6 Oe, the same as for uncoated sensor strips. This field strength has been shown to be easily achievable with a magnetised $40 \mathrm{~mm}$ spring steel strip.

\subsection{Construction of a Prototype System for Humidity Measurements}

From the results of the laboratory based impedance measurement on the humidity response of these sensors strips, it was now attempted to implement a portable 
prototype sensor system that can be used in real-world applications. This consisted of the following steps:

- Integration of a PVA coated sensor strip with a permanent magnet.

- Calibration and implementation of the hand-held reader described in Chapter 3.

- Measurement in typical applications of such a sensor system.

\subsubsection{Design of Sensor Element}

The PVA coated Metglas strip was shown to have the better humidity response so this was chosen as the sensor strip. This strip was attached to a magnetised steel strip of the same length and width $(40 \times 13 \mathrm{~mm})$ for biasing purposes.

The sensor strip was found to have largest signal in DC fields of 5.6 Oe in previous testing with the Helmholtz coil, so the steel strip was attached to the coated Metglas strip at a distance which gives this field.

This was achieved in similar fashion to Section 4.4 where the sensor strip was attached to the biasing magnet with a spacer in between, which was made from FR-4. The width of the spacer was about $3 \mathrm{~mm}$ and had a height of $8.5 \mathrm{~mm}$. This distance gives the required field strength of 5.6 Oe as shown in Eq. 4.4 .

The spring steel strip was initially coated with clear enamel spray paint to prevent rusting, and the corners were rounded with a file for safety as they were very sharp. The parts were again glued together with cyanoacrylate based glue, and the resulting sensor is shown in Fig. 4.25. Note that a sticker with a "+" sign is attached to the north pole of the magnet, and a "-" sign is attached to the south pole. This is intended to aid when re-magnetising the strip, if needed. 


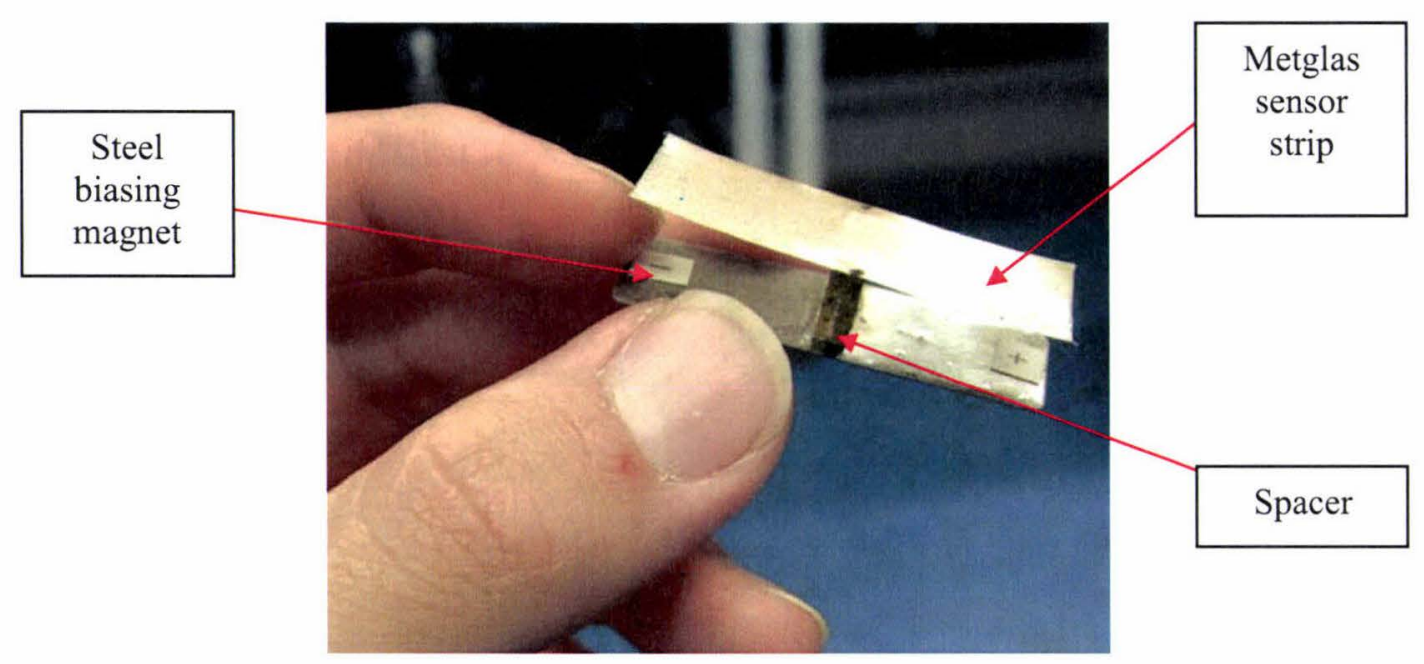

Figure 4.25: The constructed humidity sensor, showing the PVA coated Metglas strip, spacer and biasing steel strip.

\subsubsection{Evaluation of Humidity Sensor Response}

The designed humidity sensor was again placed inside the humidity controlled glass test cell and this test cell was placed inside a reader coil connected to the impedance analyser. Impedance de-tuning was performed to measure the resonant frequency against relative humidity as measured by the IC sensor. The results are shown in Fig. 4.26 .

As the plot of Fig. 4.26 shows, the constructed sensor using the steel strip had a similar slope of relative humidity against frequency as the strip with Helmholtz biasing arrangement. However, examination shows that the frequencies have been shifted up by approximately $2-3 \mathrm{kHz}$, possibly due to less uniform field compared to the Helmholtz coil arrangement. A similar effect was observed in Fig. 4.9, where the uncoated Metglas strip showed an increase in resonant frequency by about $1 \mathrm{kHz}$ for a given biasing field.

A more interesting point was that the relative signal strength (calculated using Eq. 4.2) tended to decrease with humidity. This effect was both observed using the Helmholtz coil arrangement as well as with the biasing magnet. This effect is shown in Fig. 4.27. A probable explanation to this effect is that the elasticity of the coating decreases when dry, and hence causes damping in the sensor strip, resulting in signal 
decrease. When the humidity is high, the elasticity increases, allowing larger oscillations. Also, similarly to Fig. 4.10, the signal strengths overall decreased when the sensor strip had an attached magnet.

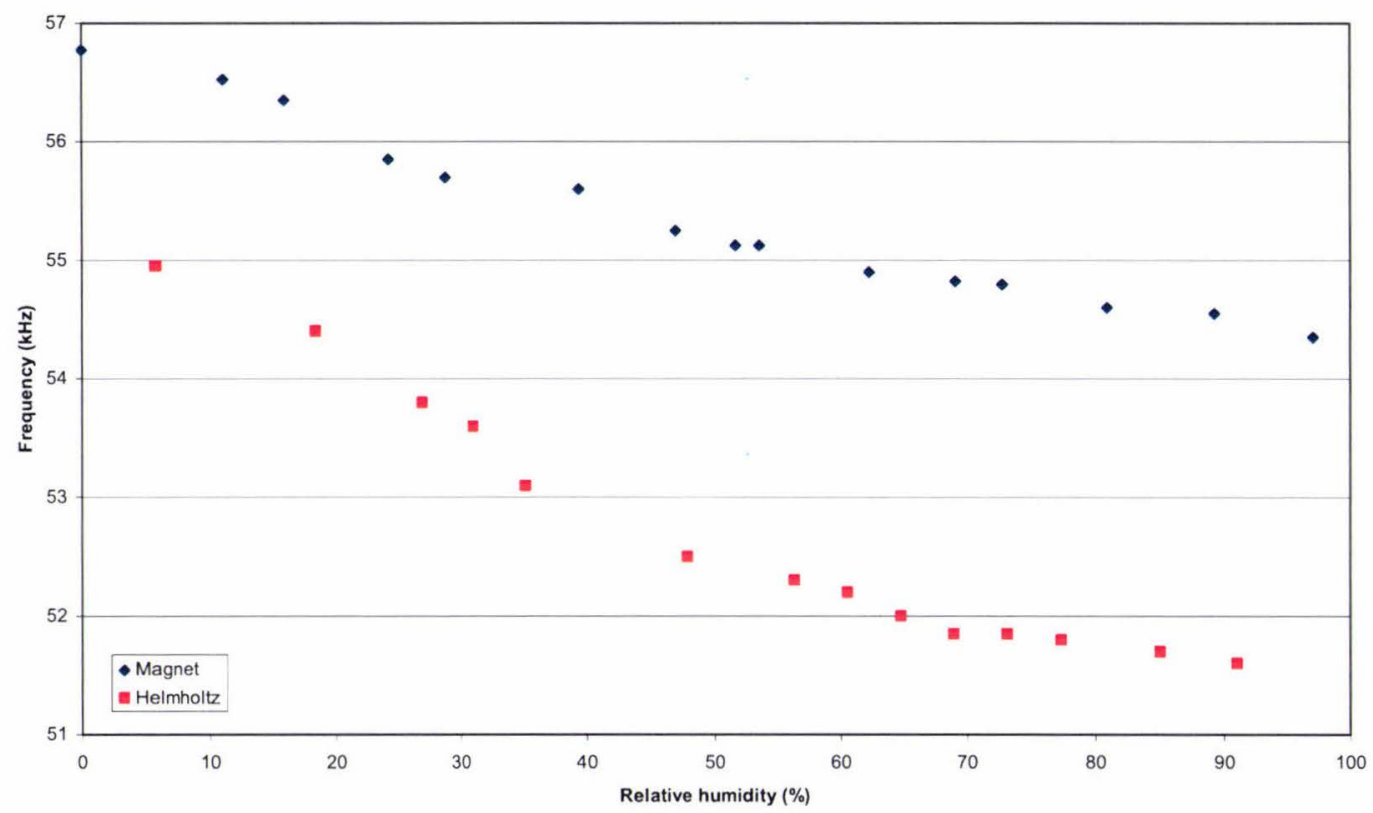

Figure 4.26: Relative humidity versus resonant frequency for the PVA coated sensor using the Helmholtz coil and biasing magnet.

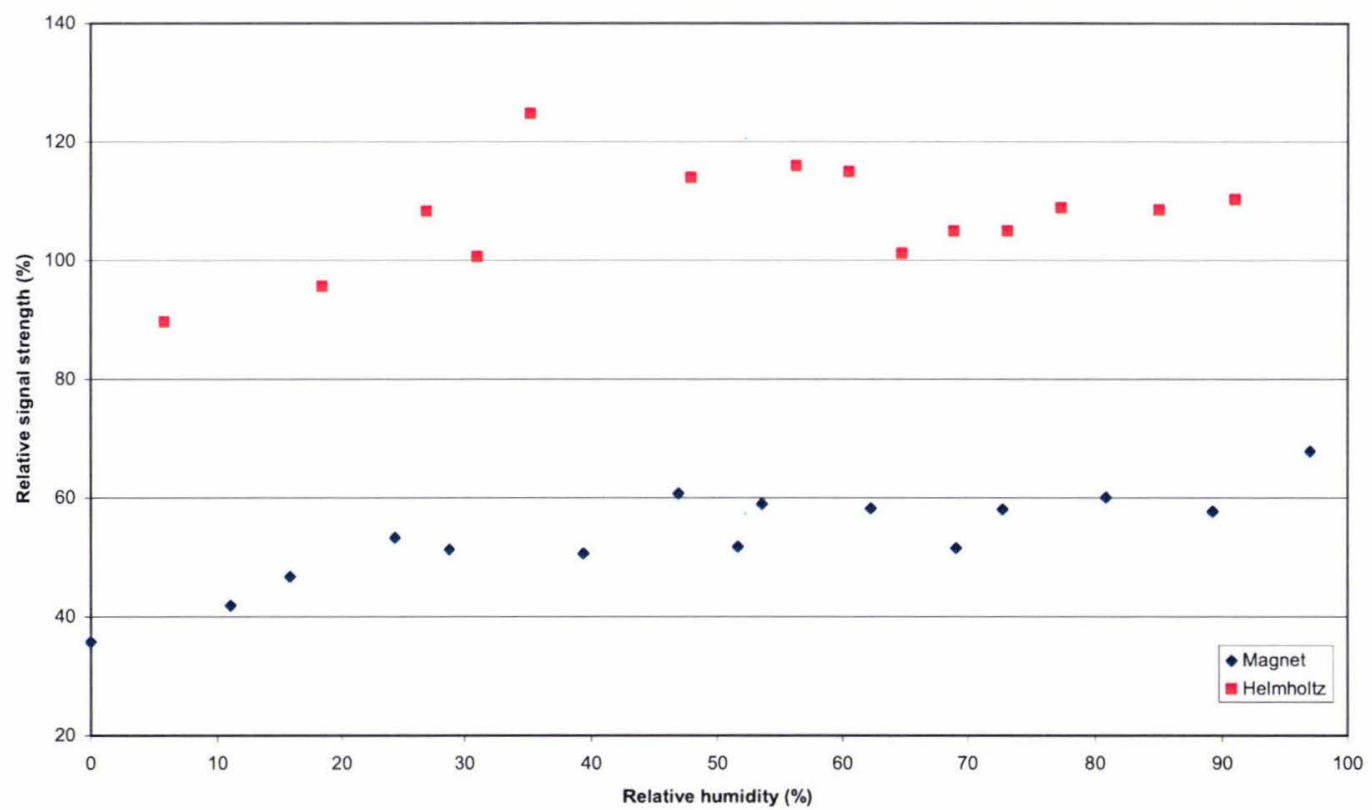

Figure 4.27: Relative humidity versus relative signal strength for PVA coated sensor using the Helmholtz coil and biasing magnet. 


\subsubsection{Using the Handheld Reader for Humidity Measurement}

The magnetoelastic sensor reader discussed in Chapter 3 was tested with the constructed humidity sensor. The humidity sensor was placed inside the glass test cell as before, and the sensor was positioned just outside $(0 \mathrm{~mm}$ distance) the reader probe, as shown in Fig. 4.28. This placement was done to ensure the system receives enough signal ensuring most reliable operation.

The signal from the reader coil, amplified and filtered by the op amp was observed on an oscilloscope to check that the system was operating correctly. The measured resonant frequency for different humidity levels was compared to values previously obtained using an impedance analyser from Section 4.8.2. The humidity levels were adjusted in the same manner as in previous experiments and the IC sensor was also used to measure the humidity inside the test cell. The results are summarised in Fig. 4.29.

The results of Fig. 4.29 shows that the resonant frequency measured by the impedance analyser using impedance de-tuning is in good agreement with values obtained with the reader. The reader was found to give slightly higher readings than with the impedance analyser. The difference between the two measurements had a maximum deviation of $304 \mathrm{~Hz}$, and this value was found at $62.25 \%$ relative humidity. The experiment was repeated and similar results were obtained.

These small differences in measured resonant frequencies between the reader and the impedance analyser may have been due to slight variation in excitation frequencies or a difference in the resolution of frequencies. The reader has excitation frequencies which are generated by a Direct Digital Synthesiser (DDS) chip, which has a constant resolution of $3 \mathrm{~Hz}$. In contrast the impedance analyser has a variable resolution which is dependant on the specified frequency range. During the measurements the frequency range was set from $54 \mathrm{kHz}$ to $57 \mathrm{kHz}$, giving a resolution of approximately $15 \mathrm{~Hz}$. 


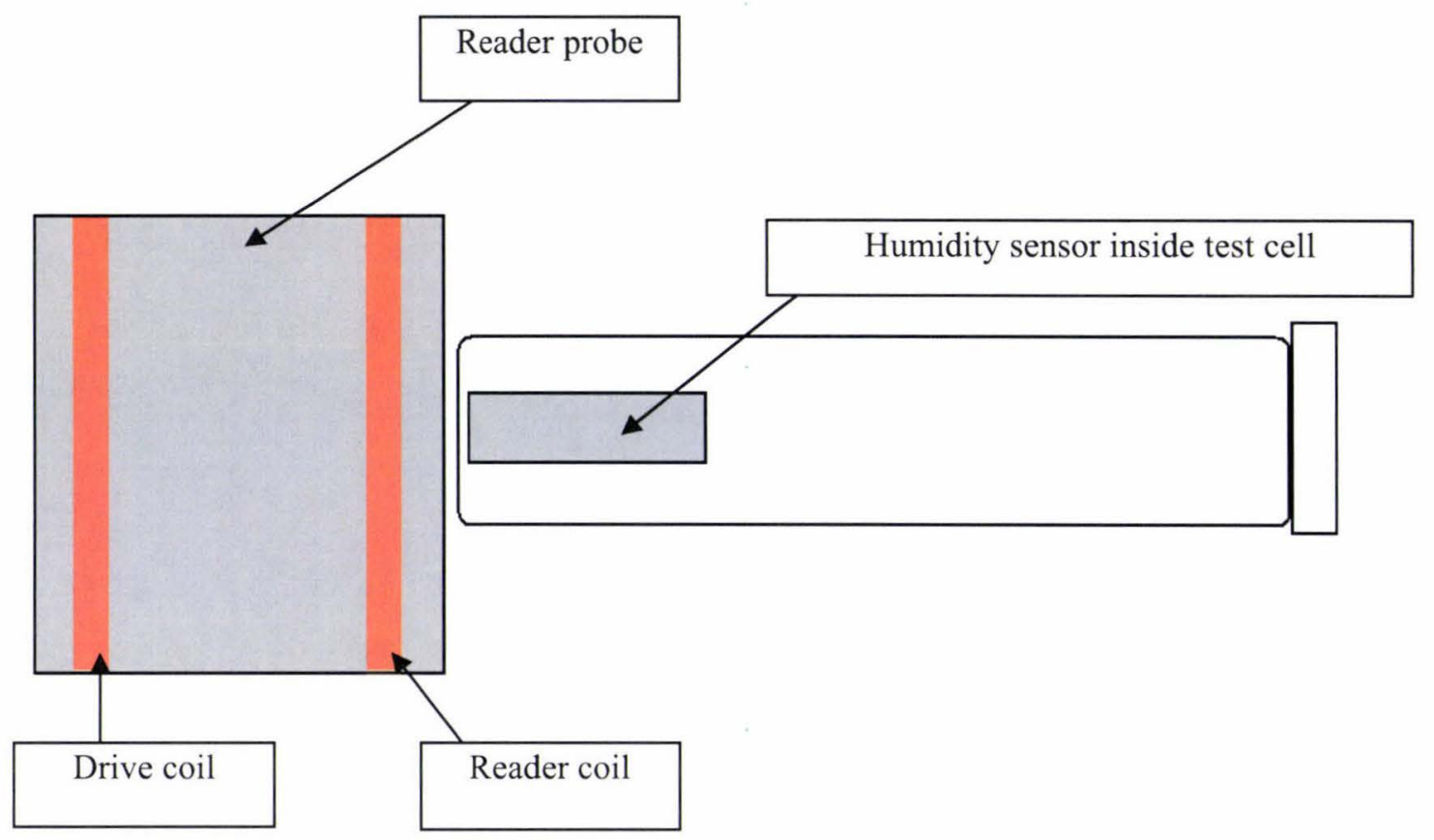

Figure 4.28: Positioning of the reader coil for the sensor reader system.

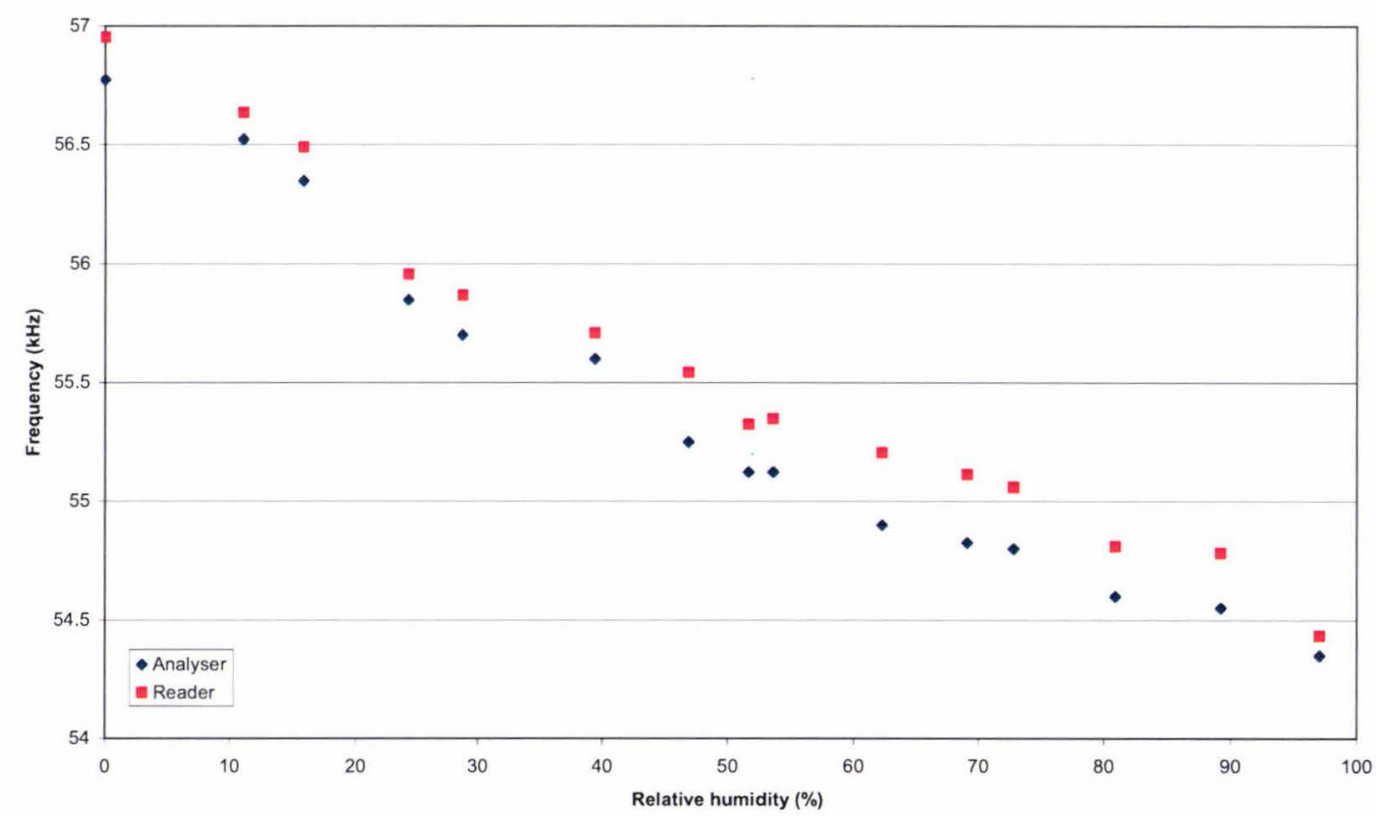

Figure 4.29: Relative humidity versus resonant frequency for PVA coated sensor using impedance de-tuning as well as the reader. 


\subsubsection{Calibration}

The experimental data from Section 4.8.3 was used to calibrate the sensor and reader for humidity versus resonant frequency. Fig. 4.29 showed that the slope for the graph is not constant for the whole humidity range. This was compensated for by using a two-line approach as shown in Fig. 4.30.

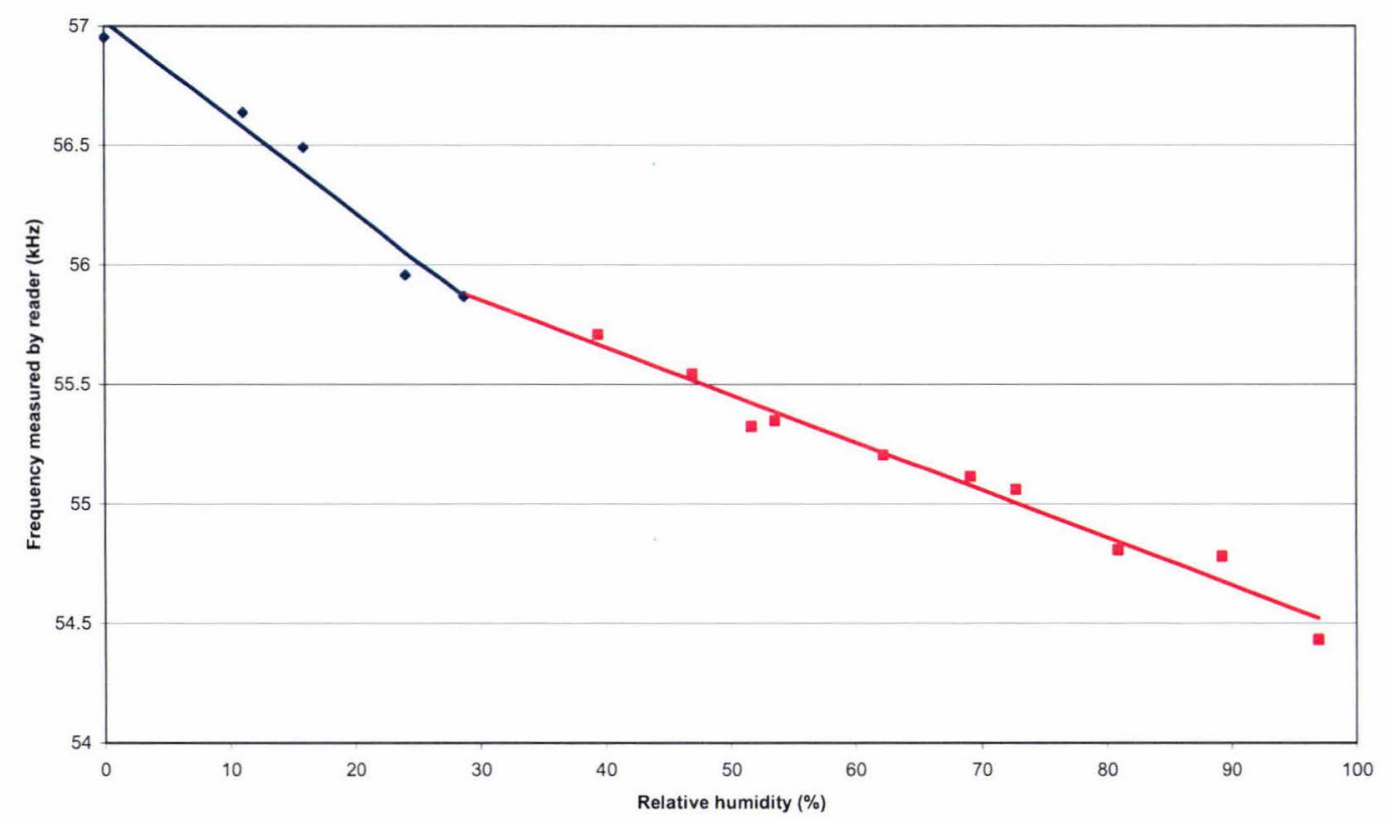

Figure 4.30: Calibrating the humidity response by splitting the graph into two sections to compensate for the different slopes.

The red line is used when the measured resonant frequency is below $55.87 \mathrm{kHz}$, which implies a relative humidity of over $28.74 \%$. The line of best fit for this range was:

$$
f=-0.0199 R H(\%)+56.45
$$

The blue line is used when the measured resonant frequency is equal to or above $55.87 \mathrm{kHz}$, implying a relative humidity of $28.74 \%$ and below. The line of best fit for this range was:

$$
f=-0.0401 R H(\%)+57.022
$$


For both cases, $f$ is in $\mathrm{kHz}$, and $R H$ is in \%.

\subsubsection{Applied Humidity Measurements}

The magnetoelastic humidity sensor system described was now used for practical applications. The first test was to measure the humidity inside a sealed plastic food container, and the second test was to measure the humidity inside a wall cavity.

\subsubsection{Plastic Food Container}

This was performed by taping the PVA coated sensor to a wall inside the container, and placing the reader probe on the top of the cover of the container giving a distance of about $1 \mathrm{~cm}$ from the end of the sensor. The Honeywell IC sensor was also placed in the container, near $(\sim 5 \mathrm{~cm})$ the magnetoelastic sensor to give reference humidity readings. Fig. 4.31 shows the measurement being performed.

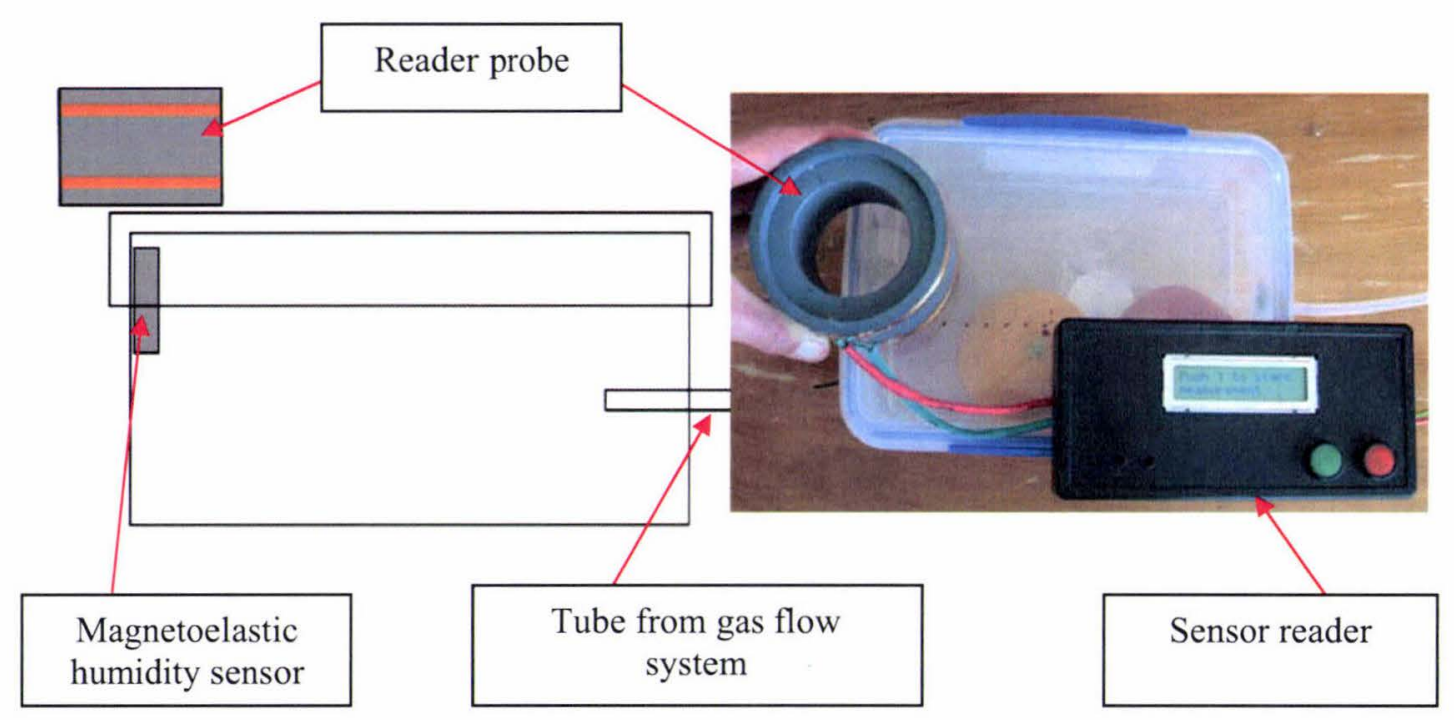

Figure 4.31: Illustration of the measurement of humidity inside the sealed food container (left) and photo (right).

The humidity inside the container was varied from $5 \%$ to just below $80 \%$ using the gas flow system described in Section 4.6. The output tube carrying the gas was placed inside the container through a small hole in the container. Humidity levels outside this 
range could not be achieved due to the larger volume inside the container and the limited flow rate from the gas flow system. Fig. 4.32 shows the plot of relative humidity values measured by the Honeywell IC sensor against the values measured with the magnetoelastic sensor system.

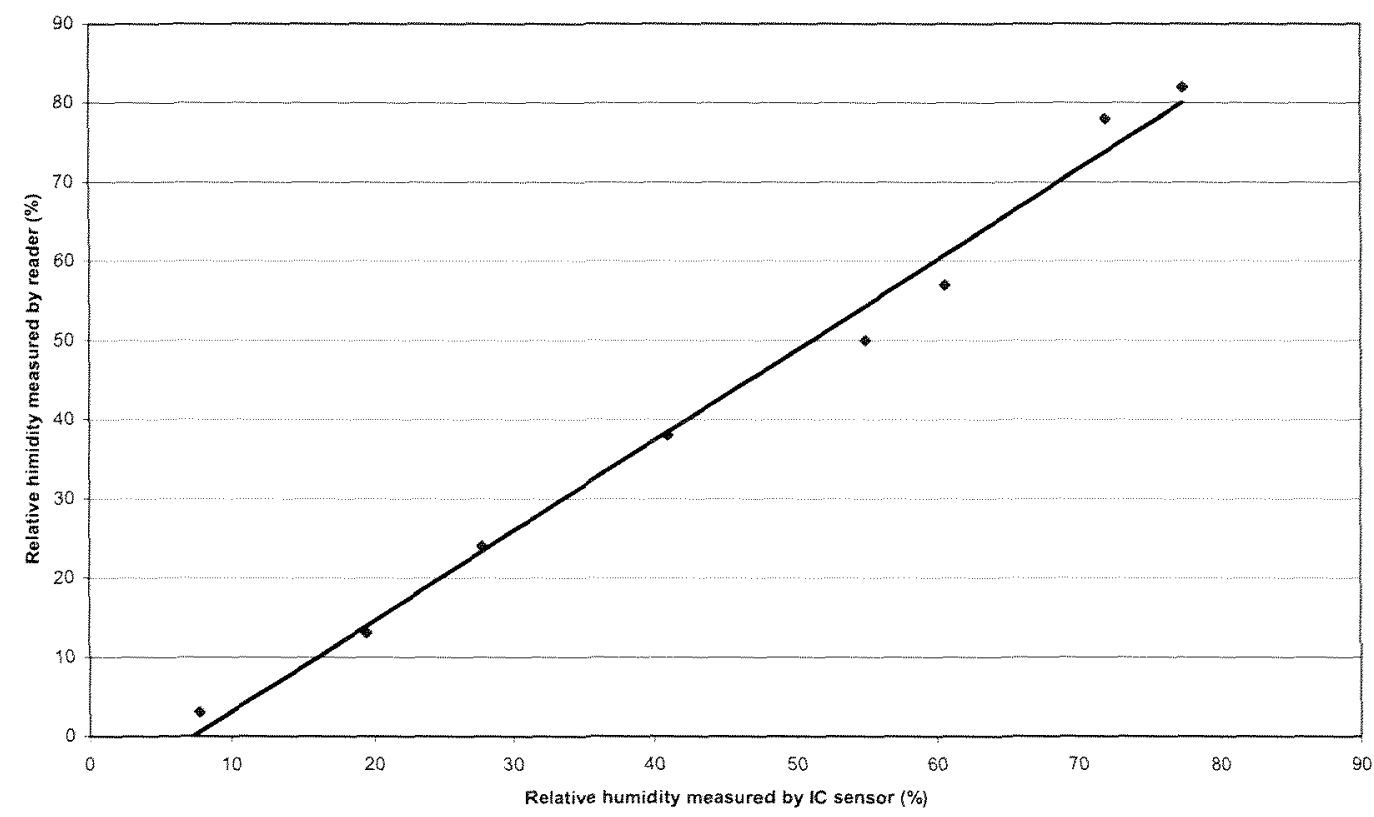

Figure 4.32: Humidity measured by IC sensor versus magnetoelastic sensor reader inside plastic food container.

It was found that the magnetoelastic sensor and reader could measure the humidity in the container to within $6.5 \%$ of the IC sensor values. In the range between $30 \%$ and $60 \%$, the difference between the two measurements was as low as $3 \%$. Fig. 4.32 also shows that at low humidity levels of under $30 \%$, the sensor system tends to underestimate the humidity.

\subsubsection{Wall Cavity}

The second measurement was to measure the humidity inside a sealed wall cavity. A model of a standard wall cavity as found in New Zealand homes was constructed, and the humidity inside was measured. The wall model is shown in Fig. 4.33. 
The wall is approximately $104 \mathrm{~cm}$ wide, $105 \mathrm{~cm}$ tall and $10 \mathrm{~cm}$ thick. One side of the wall is made of standard GIB board, about $1 \mathrm{~cm}$ thick, while the other is removable and is made of a thin wooden sheet. At the centre is a small shelf, and the sensor was placed on this shelf and taped into place, as shown in Fig. 4.34. The sensor was thus read through the $1 \mathrm{~cm}$ thick GIB board.

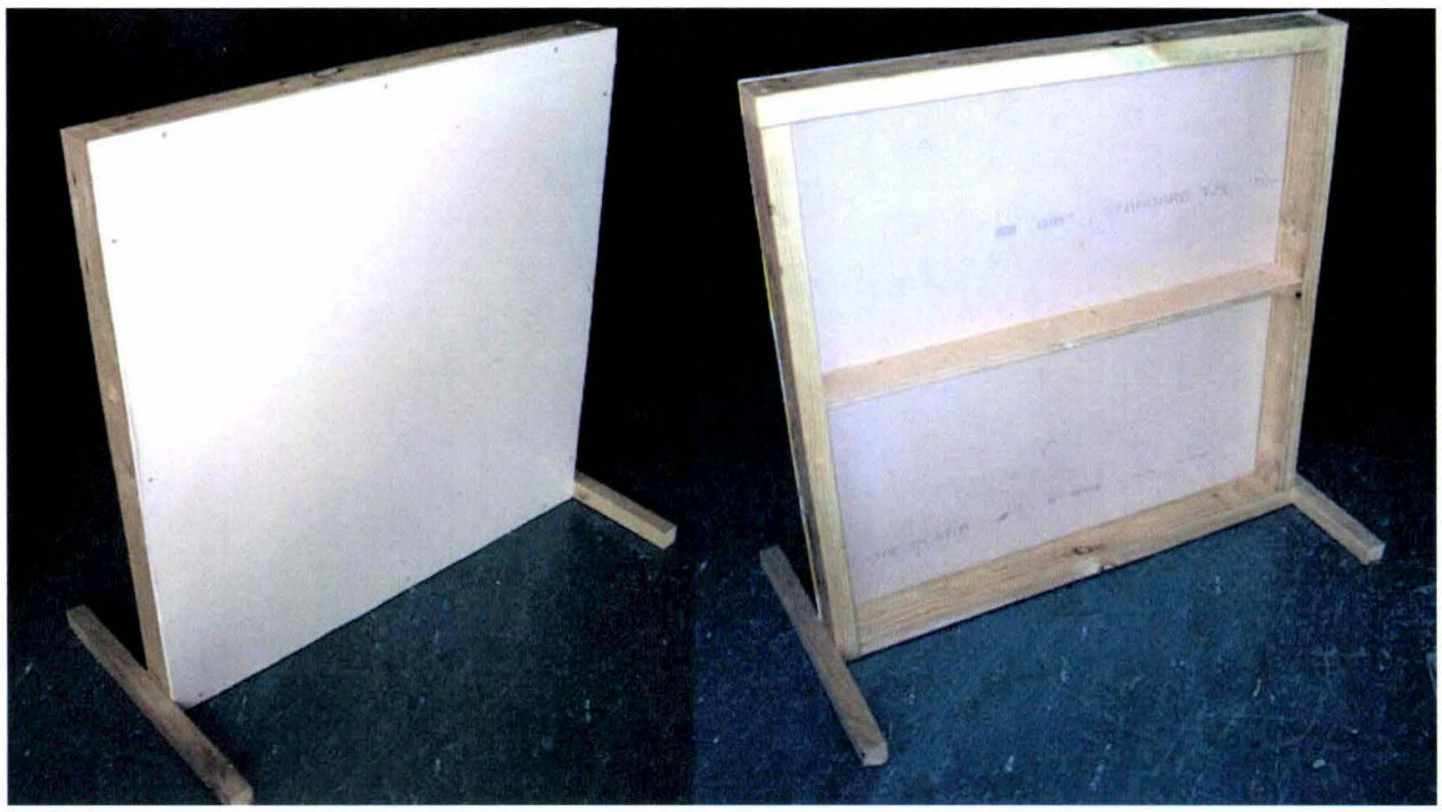

Figure 4.33: Constructed wall model for practical humidity measurement, showing the outer wall with GIB board (left), and internal view (right).

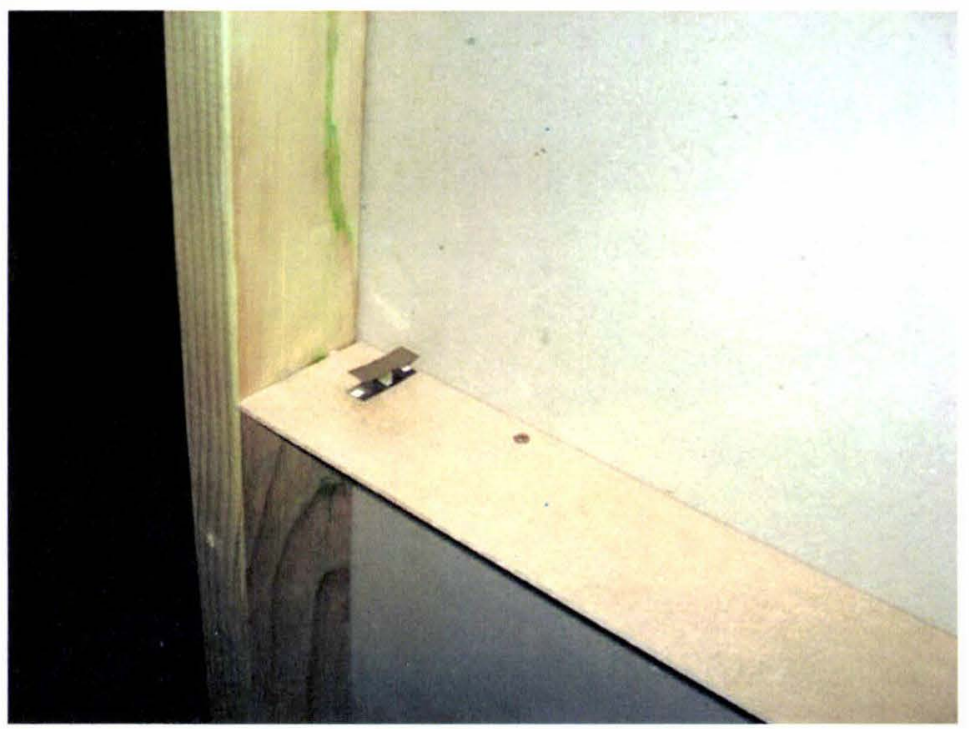

Figure 4.34: Humidity sensor placed inside the wall cavity. 
Since the Metglas strip cannot be touching the surface of the wall, the sensor had to be positioned slightly further away. An estimate of the distance from the end of the sensor to the end of the reader probe was about $1.5 \mathrm{~cm}$. The Honeywell IC sensor was placed close $(\sim 5 \mathrm{~cm})$ to the magnetoelastic sensor and with this the reference humidity measurements were made.

The wall was closed and the reader probe of the sensor reader was placed next to the wall where the sensor was placed on the other side. A wooden table was placed next to the wall so that the reader and probe could be placed on a flat surface. The measurement arrangement is shown in Fig. 4.35.

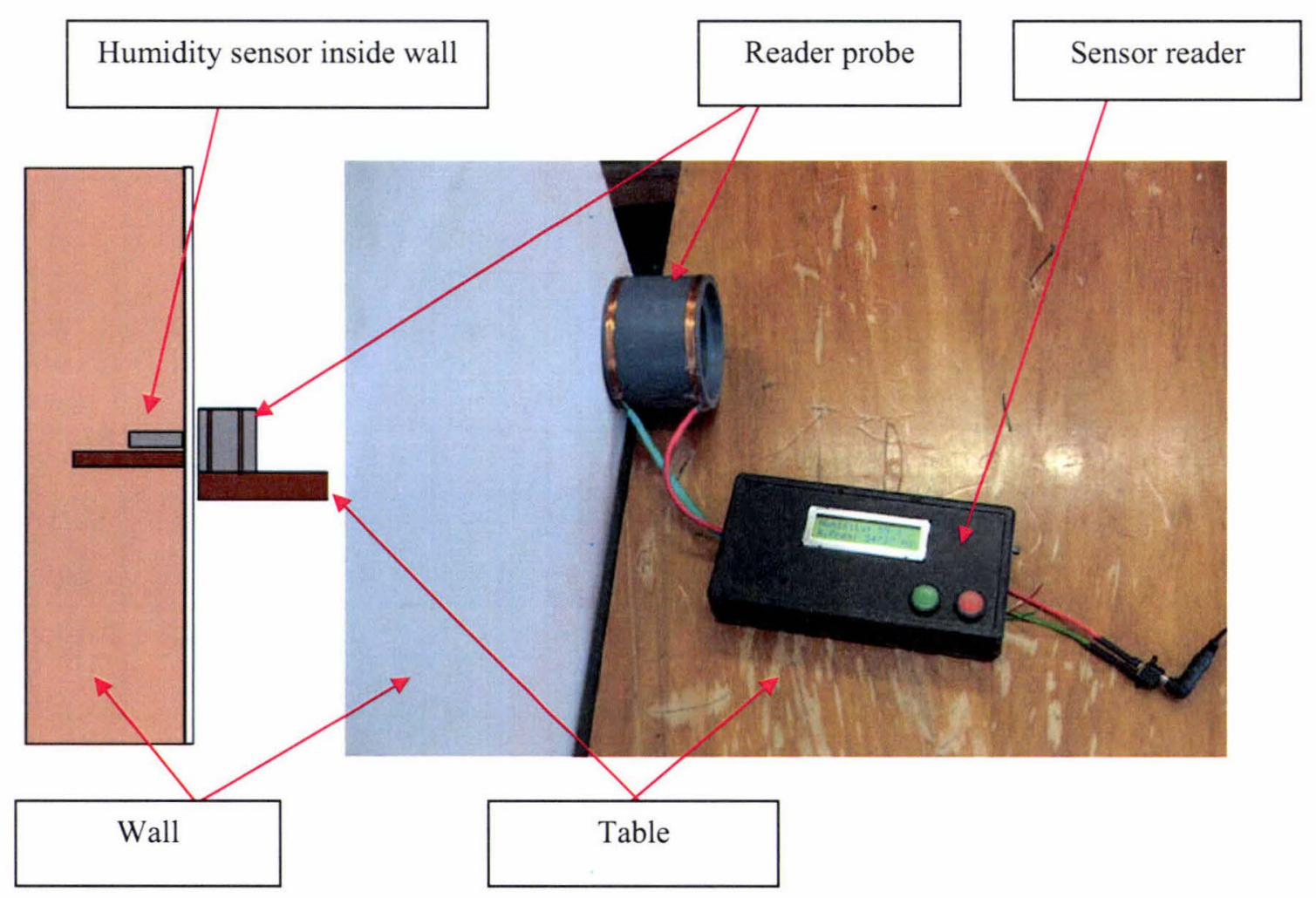

Figure 4.35: Illustration of the measurement of humidity inside the wall cavity (left) and photo (right).

The output of the op amp in the reader was viewed on an oscilloscope to ensure that the reader was operating correctly. On the oscilloscope, it could be seen that the reader was receiving enough pulses from the sensor, being read through the GIB wall. Because of the large volume inside the cavity, the gas flow system previously used 
could not be used to create different humidity levels inside the wall. In order to create humidity levels higher than the room humidity, cups of hot water were placed near the sensor and the cover was closed. This allowed relative humidity levels close to $70 \%$. The results are shown in Fig. 4.36.

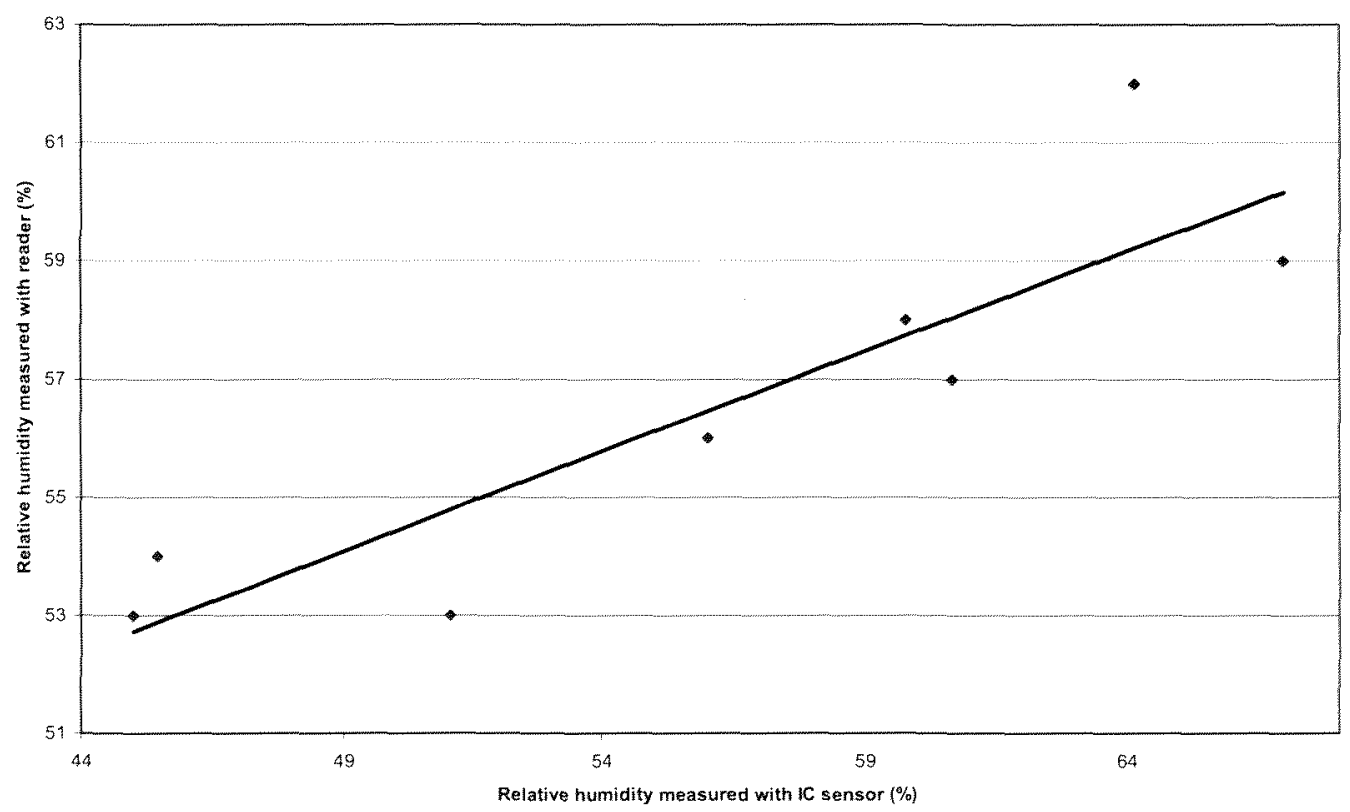

Figure 4.36: Humidity measured by IC sensor versus magnetoelastic sensor reader inside wall cavity.

The reader was able to detect the change in humidity to within $4 \%$ of the IC sensor values in the range of $51-64 \%$ relative humidity. Outside this range, it was within $8.5 \%$, which was found to be a larger variation compared to previous results. A number of reasons to the larger variation are possible. One is that the volume inside the wall cavity is large so that the humidity is not uniform inside the cavity. This would then lead to a different humidity near the two sensors, and thus a different reading.

Another possibility is that the sensor was not aligned to the centre of the reader probe correctly. In Section 3.13.2 the effect of offsetting from the centre of the reader coil was investigated. The result from Section 3.13.2 showed that if the sensor is offset by more than $1 \mathrm{~cm}$ from the centre of the reader coil the spread is increased, and this 
could have been one of the reasons for the larger spread. The difficulty is the fact that the sensor cannot be seen inside the wall cavity.

Another possibility is the increase in temperature due to the addition of the hot water. During the measurement the temperature inside the cavity was found not to increase by more than $3{ }^{\circ} \mathrm{C}$. In Section 4.4 it was shown that uncoated magnetoelastic sensors with an attached biasing magnet have some temperature dependence. However since the increase in temperature was observed to be small, the effect from this effect should have been minor, unless the PVA coating has some significant temperature dependence.

Another point which would have been highly possible is that since the magnetoelastic sensor responds much slower compared to the IC sensor, there is a lag between the humidity measured by the IC and magnetoelastic sensors. This coupled with the fact that the humidity starts decreasing rapidly with the decrease in temperature of the hot water, could have had some effect.

\subsection{Sensor Stability}

The constructed humidity sensor was found to be highly stable and reusable for over a couple of months. However, after about 5 months, the rough surface of the sensor (the side which was coated with PVA) started to turn brown, as shown in Fig. 4.37. Viewed under a microscope, the brown areas were in fact small dots on the sensor surface. The brown spots were confirmed to be rust, meaning that the PVA caused moisture to become trapped between the coating and Metglas surface resulting in corrosion of the surface of Metglas strip.

Not only did this cause the appearance of the sensor strip to change, but it also had the effect of changing the humidity response of the sensor, as well as decreasing the signal strength. In Fig. 4.38 it can be seen that the aged sensor has a lower resonant frequency compared to its new performance. It would appear also that this frequency shift is enhanced at higher humidity levels. If then the signal strength of the new sensor is compared to that 5 months later as in Fig. 4.39, it was found to be 
considerably reduced in the aged sensor. The result of this signal reduction was such that it became difficult to measure using the handheld reader in low humidity levels.

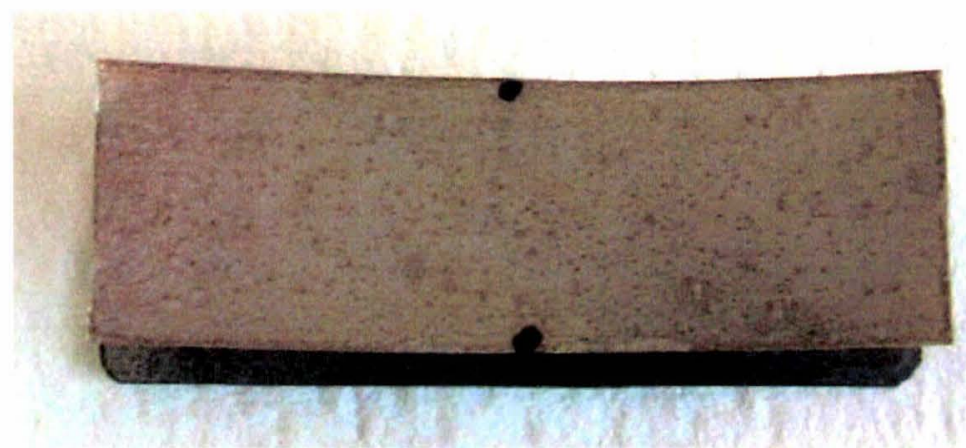

Figure 4.37: Corrosion of the sensor strip. The black spots are markers which were used to aid in attaching the spacer at its central point.

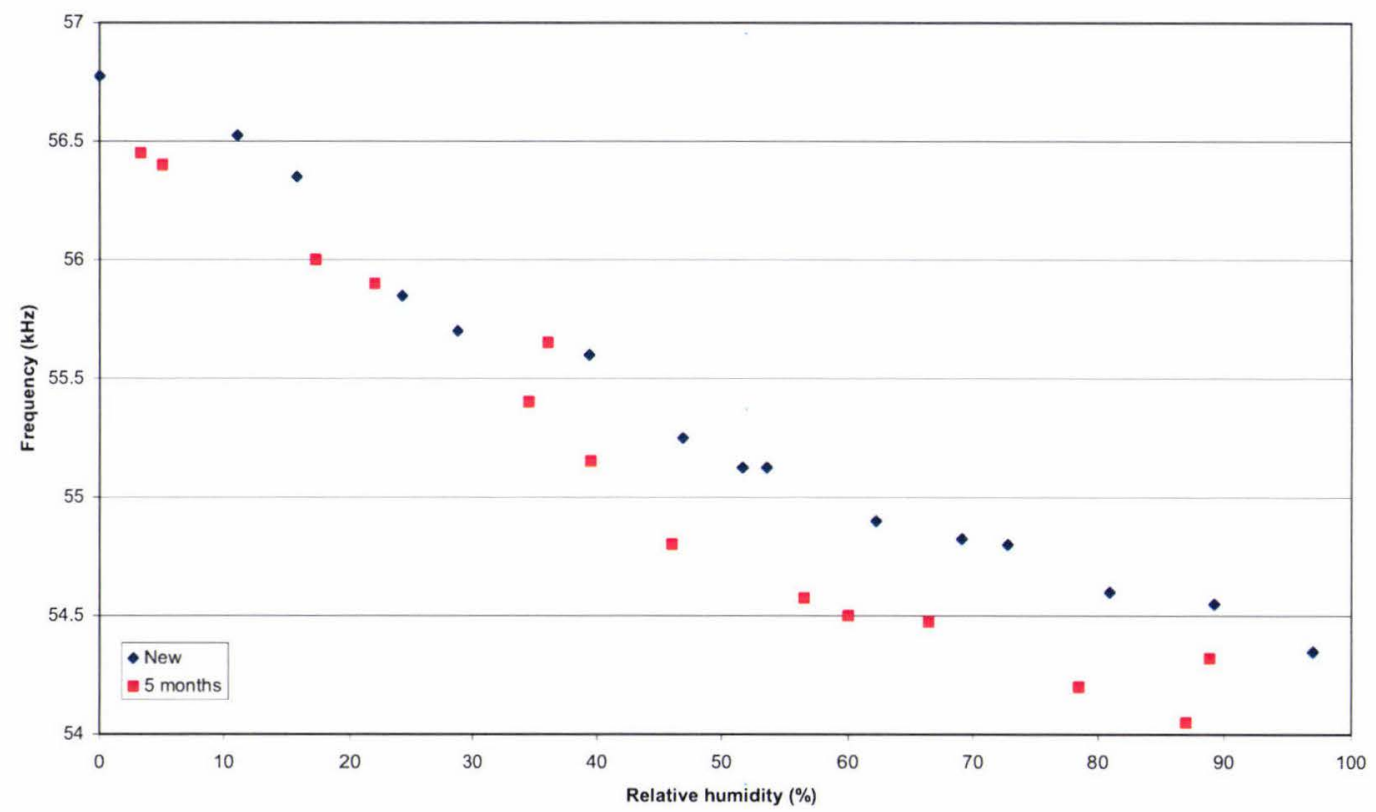

Figure 4.38: Relative humidity versus resonant frequency for a new sensor and an aged sensor. 


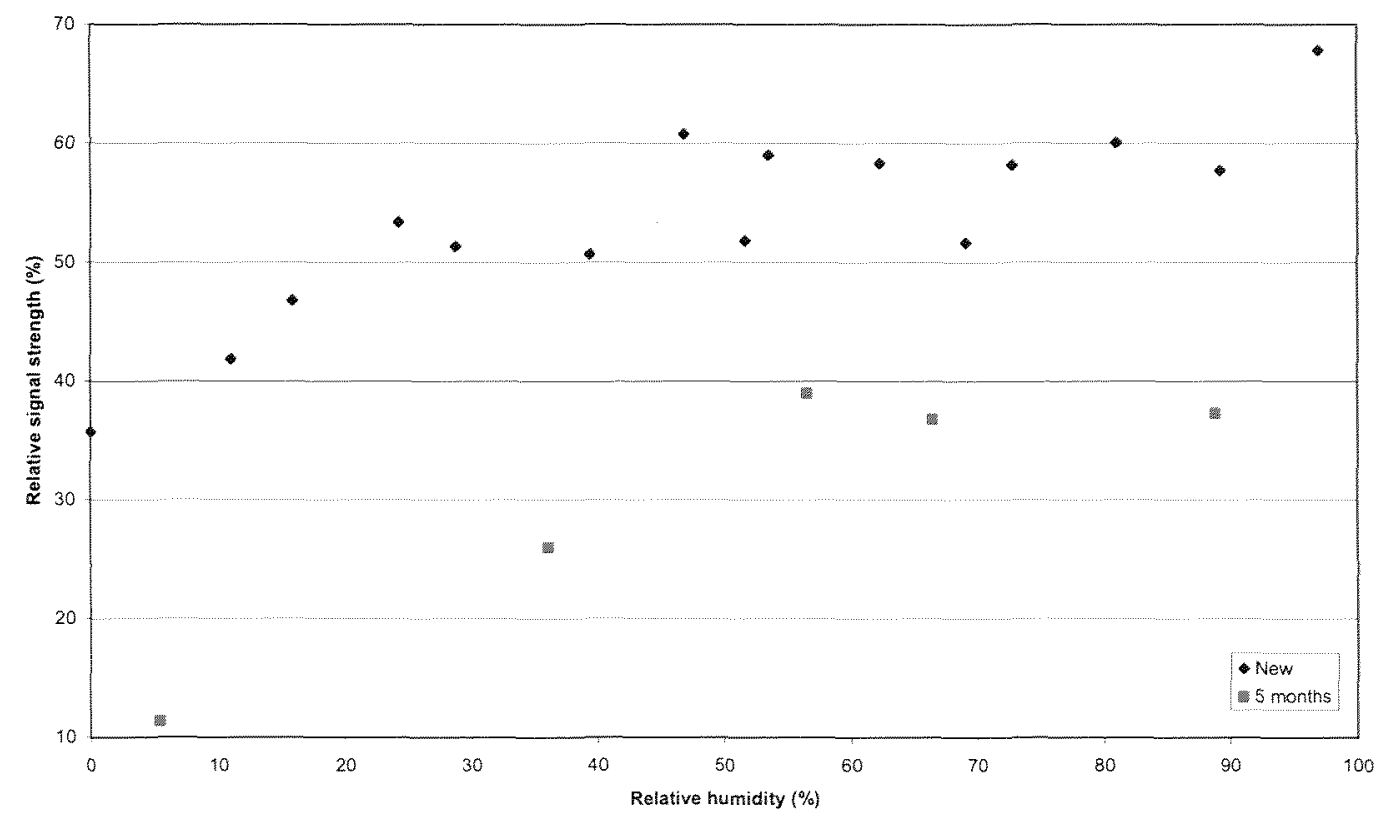

Figure 4.39: Relative humidity versus relative signal strength for a new sensor and an aged sensor.

To prevent this change in response and increase stability over time, a number of options are available [51], although they are out of scope for this project. One option is to add a passive layer to the sensor either by deliberately causing the Metglas strip to corrode under controlled conditions or by applying a top layer of a material forming a moisture and oxygen impermeable layer. The potential disadvantage of this method is that this could cause further damping of the strip resulting in signal decrease.

Another option is to use a conducting polymer such as polypyrrole or polyaniline as the coating. A coating such as this could double as a passive layer as well as a moisture absorbent layer. In Sections 4.5.2 and 4.7.2 it was shown that the Metglas was coated with polypyrrole and tested, but the response of the sensor was not as good as with the PVA, having smaller shift in resonant frequency per change in relative humidity as well as saturation at humidity levels over $35 \%$. The solution was also of low viscosity and therefore was more difficult to coat so it was not used in the final design. 
The third option is to add materials which will change the properties of the PVA film. An example is some calcium silicate material, which would prevent the formation of hydroxide and proton species. The disadvantage may be that it may alter the response of the sensor significantly, perhaps increasing the response time or reducing repeatability.

\subsection{Construction of a Magnetoelastic Sensor Array}

All previous work has been with a single strip of the sensor material. If a sensor array which has two or more strips joined together is constructed, a number of parameters can be monitored at the same time. In literature sensor arrays have been used to simultaneously monitor a number of parameters such as temperature and $\mathrm{pH}$ [57] or temperature and pressure [58]. An additional advantage is that it can be used to construct a cross-selective array, meaning that different interface coatings can be used to construct and array with a unique response to a target analyte [59]. Another motivation for production of a sensor array was that it would allow significantly smaller sensor elements than those evaluated to this point.

In order to construct such a device, methods to accurately shape the Metglas strips to a desired shape was investigated. In literature techniques such as electro-discharge micro-machining (EDM) [57] [58] have been used to create small sensor arrays accurately but because convenient access to such a system was not available, the printed circuit board (PCB) facility was used. The sensor shape was designed in software PCB design environment Altium Designer, and then two methods to shape the material, namely milling and etching, were investigated.

The shape to be constructed was a four element sensor array with elements $10 \mathrm{~mm}$, $8 \mathrm{~mm}, 6 \mathrm{~mm}$ and $4 \mathrm{~mm}$ long and $2.5 \mathrm{~mm}, 2 \mathrm{~mm}, 1.5 \mathrm{~mm}$ and $1 \mathrm{~mm}$ wide respectively. The four elements are attached to a $2 \times 2 \mathrm{~cm}$ square frame, and the designed array in Altium Designer is shown in Fig. 4.40. 


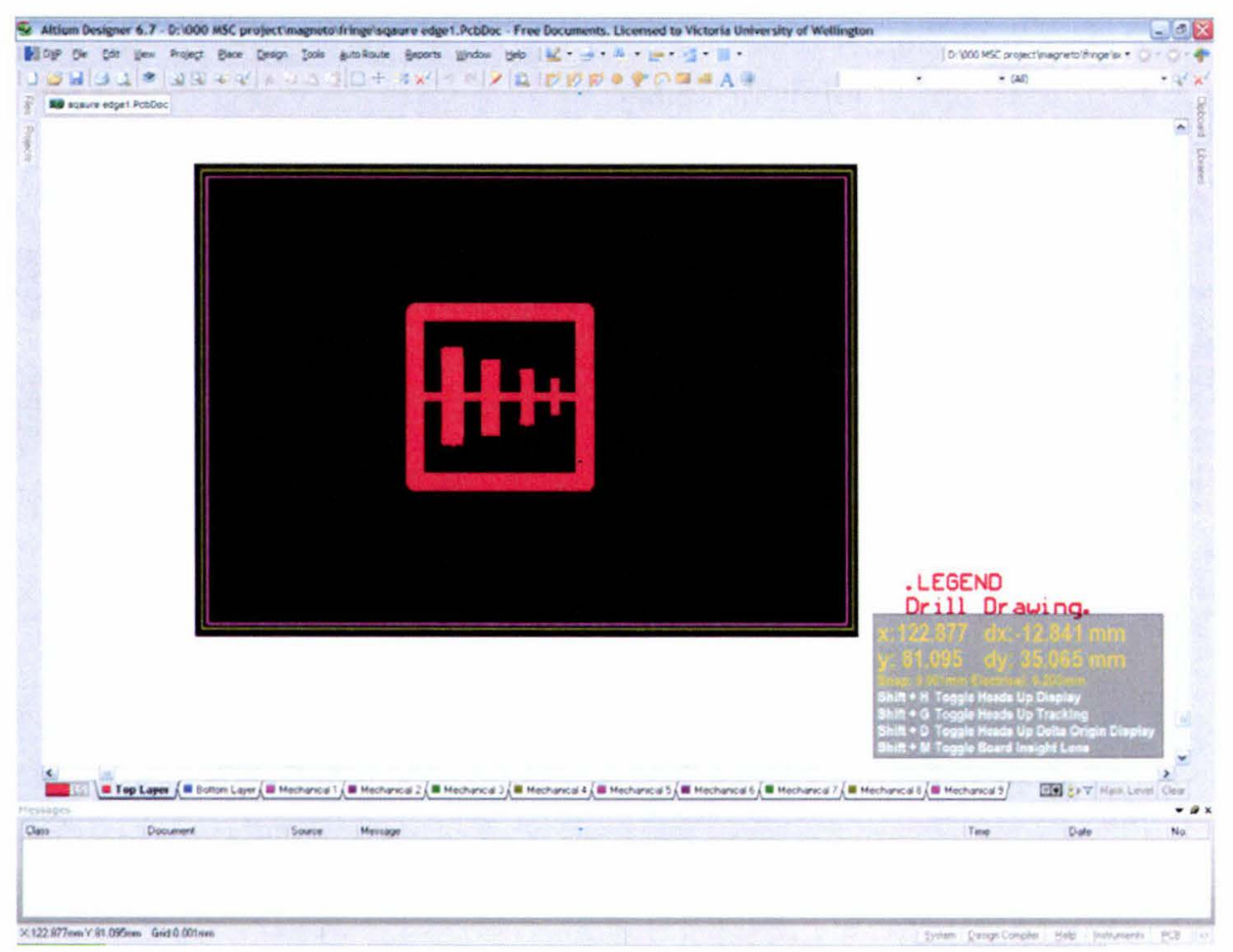

Figure 4.40: Four element sensor array design in Altium Designer.

\subsubsection{Milling}

Milling involves the use of small drilling and cutting tools which is rotated about a spindle axis. The object to be shaped is secured with a vacuum and clips onto a moving table inside the machine, and the cutting tool is moved above the object. The milling process is computer controlled and is done automatically.

The ribbon of Metglas 2826MB was put in the milling machine (shown in Fig. 4.41) in order to cut the material to shape. This was done by first by attaching the material to a spare copper board with a clear adhesive sheet, and using a $0.2 \mathrm{~mm}$ universal cutter tool on it. This was largely unsuccessful because the Metglas strip would bend and lift up where the cutter had come through. An example of a failed attempt is shown in Fig. 4.41.

Some mounting wax was then used to stick the material more securely to the backing copper board, but this was also not effective because the wax would not hold on to the material well enough, and as the cutter came in, the material would fall off. This 
method was attempted a number of times but it was concluded that it is not possible to cut such a material with a milling machine.
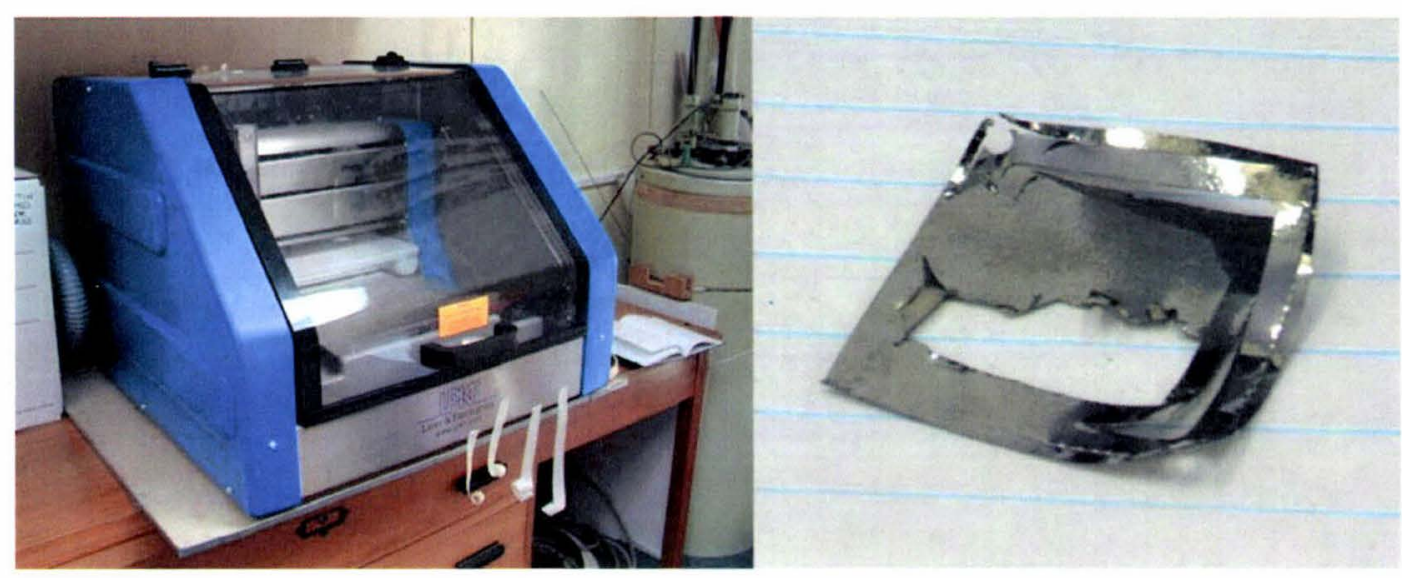

Figure 4.41: PCB milling machine (left) and a failed attempt at cutting Metglas 2826MB with the machine (right) - the material has been bent and the smaller details have been torn off.

\subsubsection{Etching}

Another method in PCB production, namely chemical spray etching was then tried on the Metglas strip. Spray etching works by dissolving unwanted metal parts off objects (usually areas of copper off a copper board) by spraying heated chemicals to the object.

Spray etching is done by printing the design onto heat transfer paper using dark, black toner with a laser printer, then transferring the design to the material to be etched by putting the printed sheet and material through a hot laminator. The sheet is then peeled off under cold running water - this transfers the black toner to the material on areas which are not to be exposed to the chemical.

The material is then put inside an etching machine such as Fig. 4.42 for a few minutes where heated (to around $45^{\circ} \mathrm{C}$ ) ferric chloride is sprayed upon it, dissolving off most metal surfaces. 


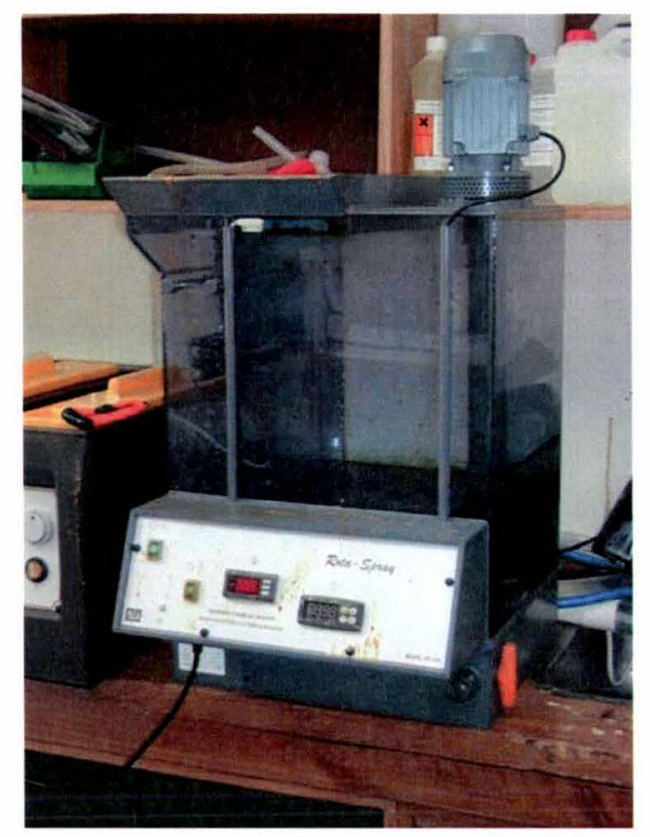

Figure 4.42: $\mathrm{PCB}$ etching machine used to shape Metglas 2826MB.

The first time this was done with the Metglas, the material was secured to a backing copper board simply by means of masking tape, and so the ferric chloride had come into contact to the back of the material, resulting in the strip becoming very thin. In order to counter this problem, mounting wax was used. The waxes used for this purpose were sourced from South Bay Technology [60]. The type which was found to work best was yellow mounting wax (MWH080), shown in Fig. 4.43 which had a high strength and relatively high melting point $\left(80^{\circ} \mathrm{C}\right)$.

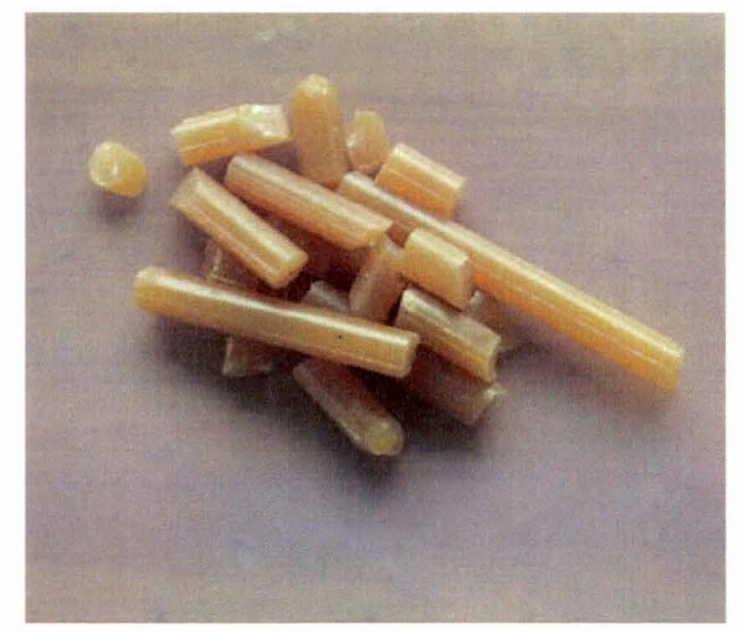

Figure 4.43: Yellow mounting wax used to secure the strip to the copper board. 
Other waxes tested unsuccessfully include a clear, very hard wax (MWH135), which was found to be difficult to handle due to its high viscosity and to remove, and paraffin wax which has a melting point of about $40^{\circ} \mathrm{C}$ so it melted inside the machine. The yellow wax was melted onto the copper board by first heating the board in an oven, then placing a small piece of solid wax to the heated board, and once melted, putting the Metglas strip on the melted wax and applying some pressure.

The copper board with the attached Metglas strip was then put in the machine in 30 second runs, after each run checking the condition. It was found that 2 runs (equalling 1 minute total) were enough to remove most of the resident material. The next step was to remove the Metglas strip from the copper board. This was done simply by gently pulling the edge of the material up with a pair of tweezers without any heating.

The strip must now be cleaned. This was achieved by putting the strip in a beaker of toluene for 30 minutes, then removing the toluene from the Metglas (as toluene is insoluble in water) by immersing it in a beaker full of drum ethanol for a further 30 minutes. The strip was then put under running water. Although this method removed most of the wax residue and printer toner, some small areas were still covered.

Some methods to further clean the strip was investigated such as putting in an ultrasonic cleaner with a beaker full of toluene for a few hours. This however was not effective as the cleaner was not powerful enough. Ultimately the strip was easily cleaned by wiping gently with cotton tips dipped in toluene or acetone.

To protect the array from physical damage and to add stability, a square frame was constructed by cutting a square hole in a used FR-4 board. The array was then taped onto this frame. The resulting sensor array is shown in Fig. 4.44. 


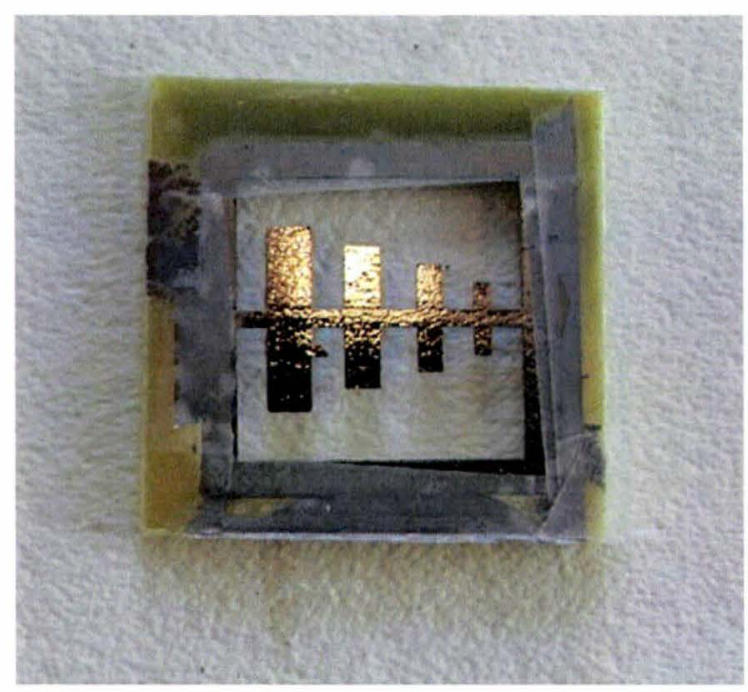

Figure 4.44: Completed Metglas sensor array using the etching process.

\subsubsection{Characterisation of Constructed Sensor Array}

The characteristics of the constructed sensor array were investigated using impedance de-tuning. The experimental setup was essentially the same as in Section 4.2 where the constructed sensor array was placed inside a reader coil connected to the HP4294A impedance analyser, and magnetic biasing was provided by a Helmholtz coil connected to the Topward DC power supply.

It was found that only the two larger sensor elements could be detected by the impedance analyser, as the signal of the smaller elements had signal strength too low and was buried in noise. The response of the array against bias field was investigated and the resonant and anti-resonant frequencies as well as relative signal strength (calculated with Eq. 4.2) for the two visible elements are shown in Fig. 4.45 and 4.46. 


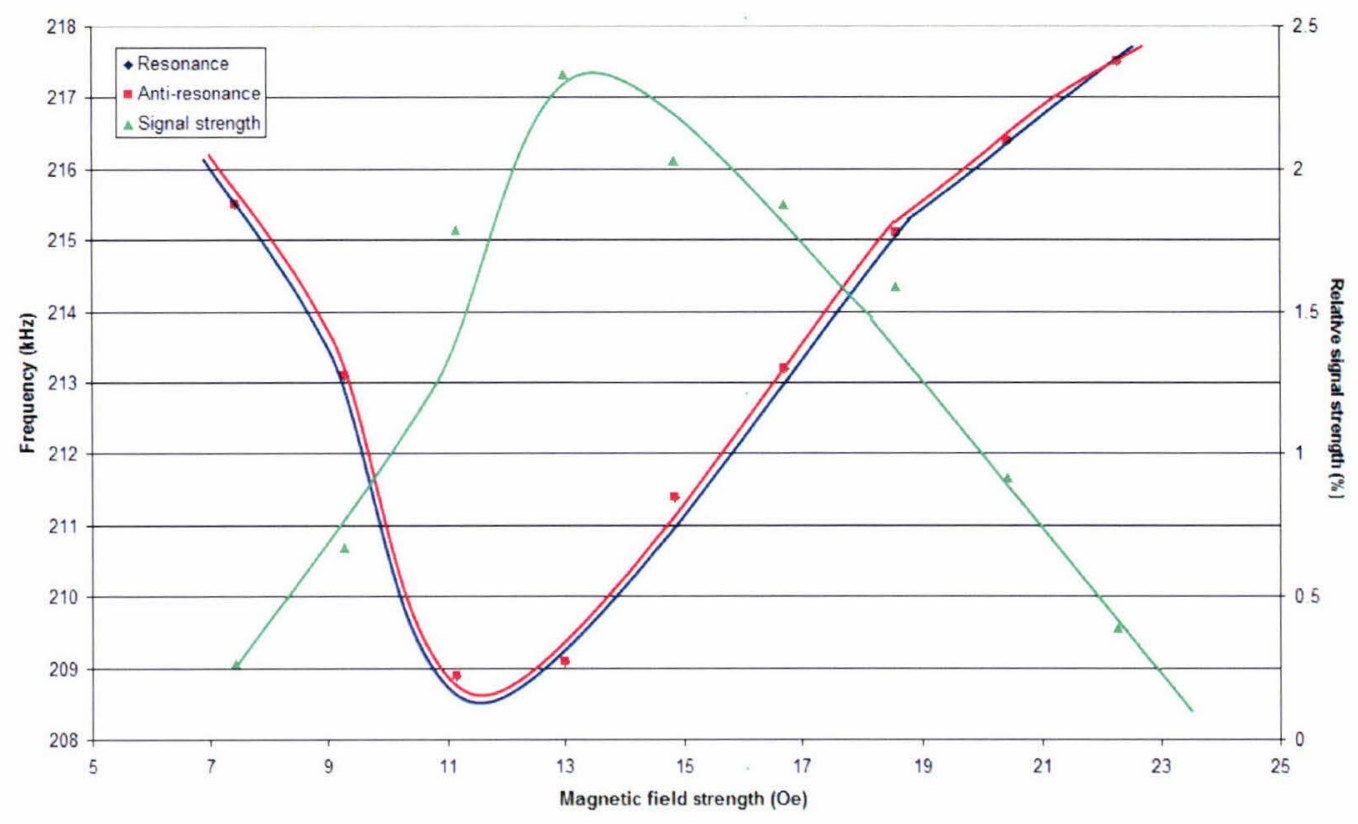

Figure 4.45: Biasing magnetic field strength versus resonant and anti-resonant frequencies as well as relative signal strength for the $10 \mathrm{~mm}$ sensor element.

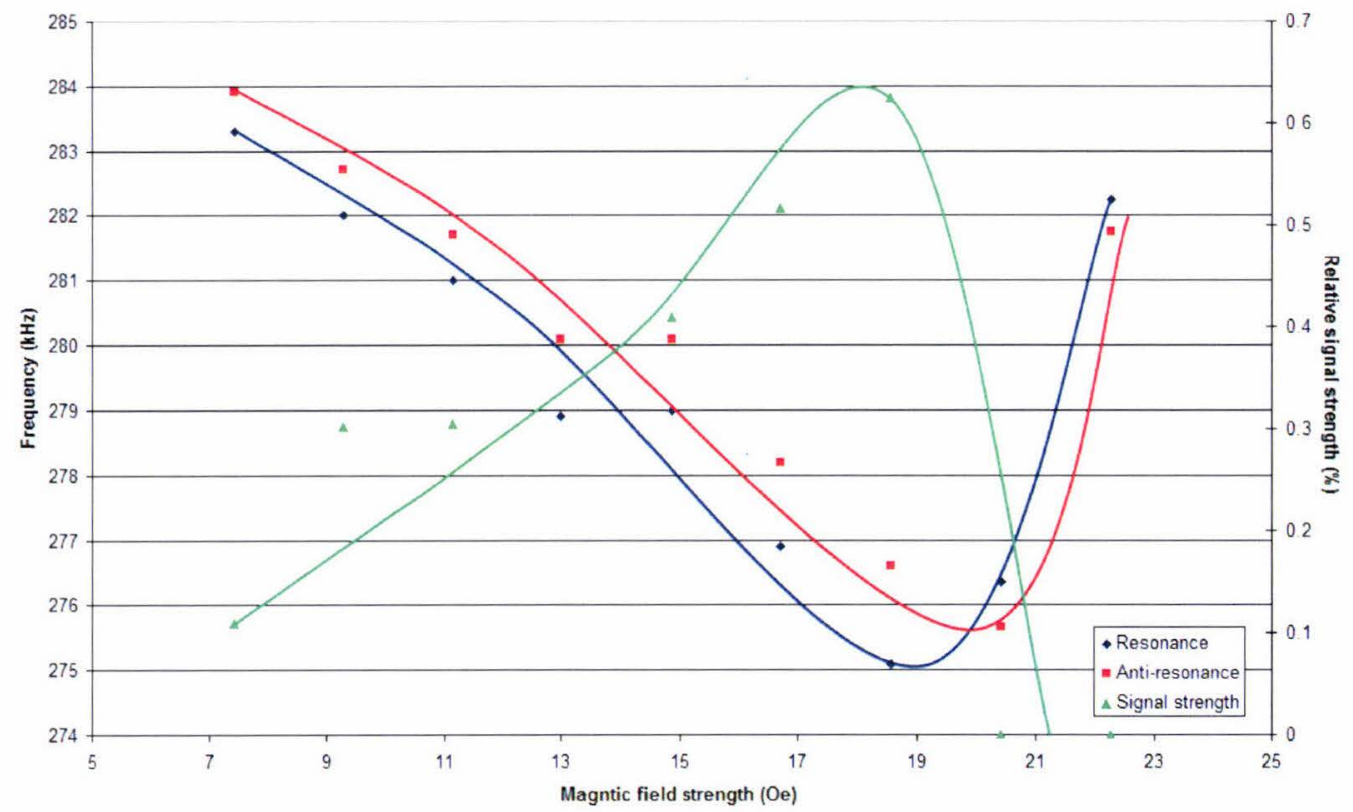

Figure 4.46: Biasing magnetic field strength versus resonant and anti-resonant frequencies as well as relative signal strength for the $8 \mathrm{~mm}$ sensor element.

It was found that for the $10 \mathrm{~mm}$ element, the optimal biasing field was about $14 \mathrm{Oe}$, and the relative signal strength at this point was just $\sim 2.33 \%$. An interesting point for the $10 \mathrm{~mm}$ sensor element was that the resonant frequencies were found to be almost 
exactly the same as the anti-resonant frequencies. For the $8 \mathrm{~mm}$ element, the optimal biasing field was found to be about $18.5 \mathrm{Oe}$, and the signal strength at this point was only $\sim 0.62 \%$. For both cases the optimal field required a relatively strong biasing field of more than $13 \mathrm{Oe}$, and the signal strengths at this point was found to be very small, making detection difficult.

\subsubsection{Discussion}

A prototype Metglas sensor array was successfully constructed by the use of the PCB facility. The original method was to use the milling machine, but this caused the material to tear during the process and so was not successful. Etching was then attempted and was found that if the Metglas strip is securely stuck onto a backing board with wax, the process is able to make accurate sensor arrays.

Impedance de-tuning was then performed on the constructed sensor array. It was found that only the two longer sensor elements could be detected on the impedance analyser - even though they could be seen, their signal strengths were very small, and it is thought that it may be difficult to detect in real-world applications.

In addition, the optimal bias fields required for these sensors were found to be quite high, with both the $10 \mathrm{~mm}$ and the $8 \mathrm{~mm}$ elements requiring more than 13 Oe for optimal operation. This means that the spring steel strip used previously cannot be used to bias these sensor arrays; a material with a higher remanence must be used. 


\section{Summary and Conclusions}

The goal of this project was the development of a prototype passive wireless sensor system and the proof of concept application of the system for the monitoring of moisture in building structures or sealed containers. At the conclusion of the project it was shown that such a system can successfully be used to monitor the relative humidity levels in sealed spaces such as building structures. The most significant results achieved during the project can be summarised as follows:

- Metglas 2826B, typically used as the sensor element in EAS applications, was evaluated for environmental sensing applications. Both the signal strength and resonant frequency of Metglas $2826 \mathrm{MB}$ magnetoelastic strips was found to be highly dependant on the biasing magnetic field strength. Through the use of a Helmholtz coil the optimal biasing field strength for a strip $40 \mathrm{~mm}$ in length was found to be $5.6 \mathrm{Oe}$, and at this point the signal strength from the strip was maximal. The temperature dependence of the resonant frequency was also evaluated, and it was found that over the range of temperatures tested $\left(18^{\circ} \mathrm{C}-60^{\circ} \mathrm{C}\right)$ the sensors did not have a significant temperature dependence, with measured gradients less than $5 \mathrm{~Hz} /{ }^{\circ} \mathrm{C}$.

- A handheld reader was constructed to replace the impedance analyser, which is expensive and inconvenient for practical use. The reader is small enough to be carried in one hand, and has a simple interface. The reader consists of a small enclosure which houses the electronics and a reader probe which consists of a drive coil and a reader coil. The measurement is done by exciting the sensor with sinusoidal bursts of increasing frequency, and measuring the length of the sensor's ring-down at each frequency. The frequency at which the ring-down is longest is identified as the sensor's resonant frequency. In testing the reader was able to detect sensors at up to $2 \mathrm{~cm}$ in distance from the probe. Placing the sensors at further distances did not result in substantial increase in spread of the measured values. Offsetting the sensor from the centre of the reader coil however was found to increase the spread in the measurement, reducing the accuracy. 
- Since the Helmholtz coil is large it cannot be used for a practical sensor system. Thus to make the system portable a permanent magnet was attached to the Metglas strip. The magnet was a piece of spring steel, and this was found to be sufficient as it could provide enough magnetic field for biasing, and could keep its magnetisation for a long time. The magnet and Metglas strip had a spacer between, which is used to minimise the contact area, and to adjust the magnetic field strength from the magnet. Placing this structure in different temperatures however revealed that it had an increased temperature dependence of $13 \mathrm{~Hz} /{ }^{\circ} \mathrm{C}$ between $20^{\circ} \mathrm{C}$ and $66.7^{\circ} \mathrm{C}$.

- The Metglas sensor strips were found to have very little humidity response without any coating. In order to make the Metglas strips sensitive to humidity, the application of two moisture absorbent coatings were evaluated. Polyvinyl alcohol (PVA) was chosen as it had much better sensitivity with changes in humidity at $50 \mathrm{~Hz} / \mathrm{RH}(\%)$ from $0-70 \%$ humidity as opposed to polypyrrole which had $22.4 \mathrm{~Hz} / \mathrm{RH}(\%)$ in a smaller range of $0-30 \%$ humidity. In addition, polyvinyl alcohol also has a moderate viscosity meaning it could be spin coated onto the Metglas strip. The disadvantage of polyvinyl alcohol was the longer response time of 1 hour instead of 30 minutes of the polypyrrole.

- The PVA coated Metglas strip was attached to a biasing magnet and it was used to measure the humidity inside a sealed food container using the constructed reader. The results showed good agreement with a commercial IC sensor, with the error being as low as $3 \%$. The sensor was then placed inside a wall cavity, and the sensor was read through a standard GIB board. The sensor could be detected, but the measured humidity values were found to show a greater variance to the IC sensor compared to previous measurements due to a number of possible factors including misalignment of the probe, non-uniform humidity inside the cavity and the difference in response times for the two sensors.

- The PVA coated sensor was highly reusable for a couple of months, however after about 5 months the surface of the Metglas became brown due to rust. 
brown due to rust. This had the effect of changing the humidity response as well as reducing the signal strength, especially at lower humidity levels.

- Attempts were also made to construct a miniaturised Metglas sensor array, using a standard printed circuit board (PCB) facility. The initial method was to use the milling machine, but this was unsuccessful because the Metglas material would bend and then tear during the process. The next method attempted was to use the etching process, and this was used successfully to construct a 4 element sensor array. This array was then investigated by impedance de-tuning, and it was found that the signal was very small, raising question to whether this could be detected in real-world applications.

Although the development of this prototype sensor system usefully showed that relative humidity levels can be accurately measured in closed spaces, some work would still be needed to produce a practical system. This work would include the following developments:

- Further development of the chemical interface layers in order to enhance the sensitivity and speed of response to moisture. However, the most important aspect of this work would be develop a layer that provides long term stability and sensitivity to the sensor and does not degrade the sensor in any way.

- Possible improvements to the sensor reader can also be done as was discussed in Section 3.14. One area of improvement is to increase the detection distance which may be achieved by increasing the signal quality. Another area is to investigate magnetic cores for the reader probe. The reader probe has a circular space for a ring shaped core which may increase the signal amplitude. Another improvement is to eliminate the reader probe by integrating a spiral inductor into the reader's PCB. This would reduce the bulk and make the system more convenient. The reader can also be interfaced with a PC using RS-232 to add further functionality. Finally, it may also be desirable to reduce the time taken for a single measurement - this may be achieved by optimisation of the code or a re-design. 
- A significant amount of testing in real building structures wired with appropriate additional sensors such as temperature and humidity sensors should then be performed in order to evaluate the long term performance of this sensor system.

- The development of such a system to other environmental sensing applications should be investigated. Possible parameters include $\mathrm{pH}$ levels, bacteria, carbon dioxide and many others as explained in literature.

- Finally, since the sensor is relatively sensitive a case should be designed for the sensor to protect against physical damage in real-world applications. The case should allow good contact with the surrounding environment and be as small and cheap as possible. 


\section{Appendices}

Appendix A: Schematic Diagrams

Schematic of magnetoelastic sensor reader:
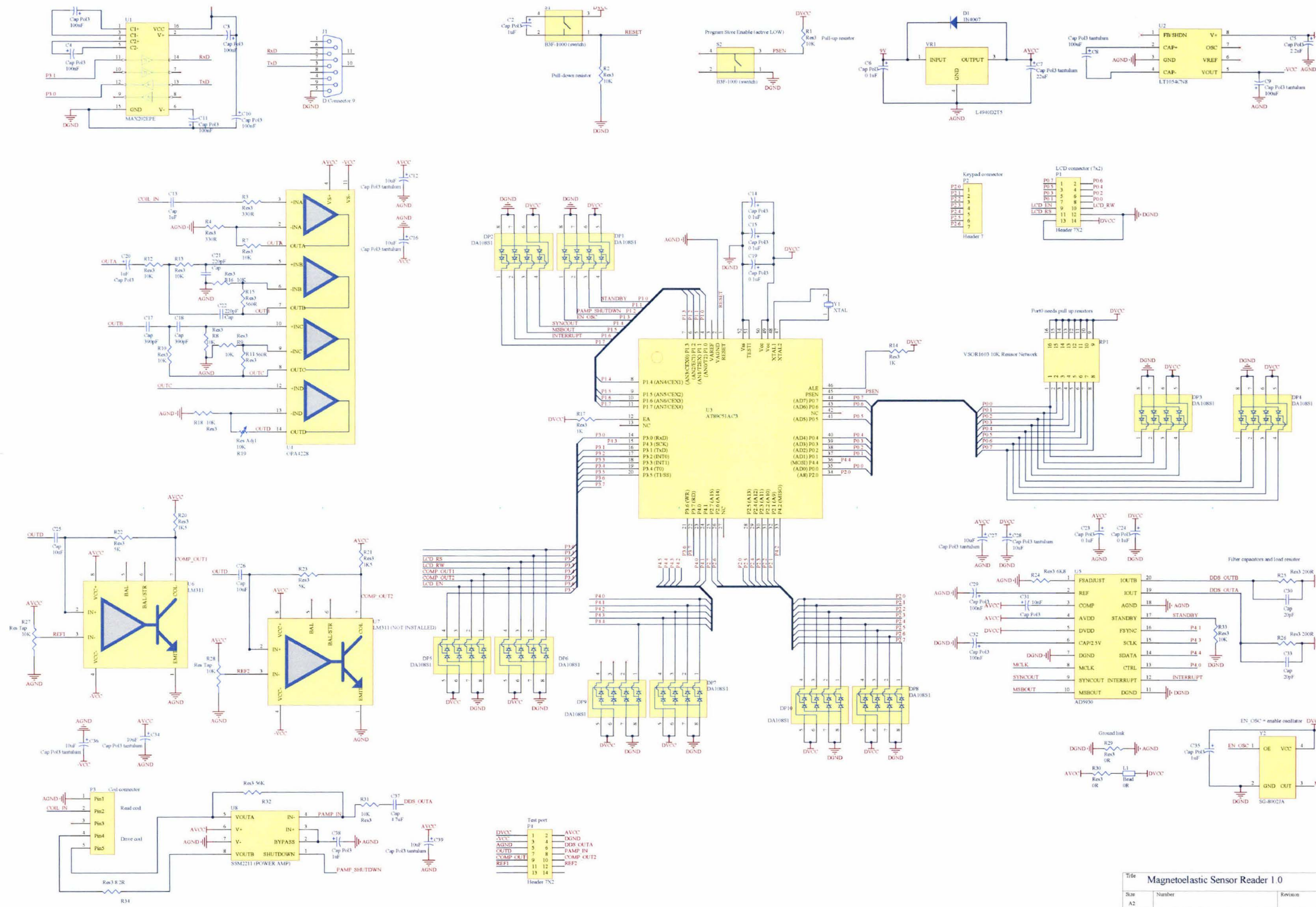


\section{Appendix B: PCB Layout}

PCB layout for magnetoelastic sensor reader - top layer (top) and bottom layer (bottom):
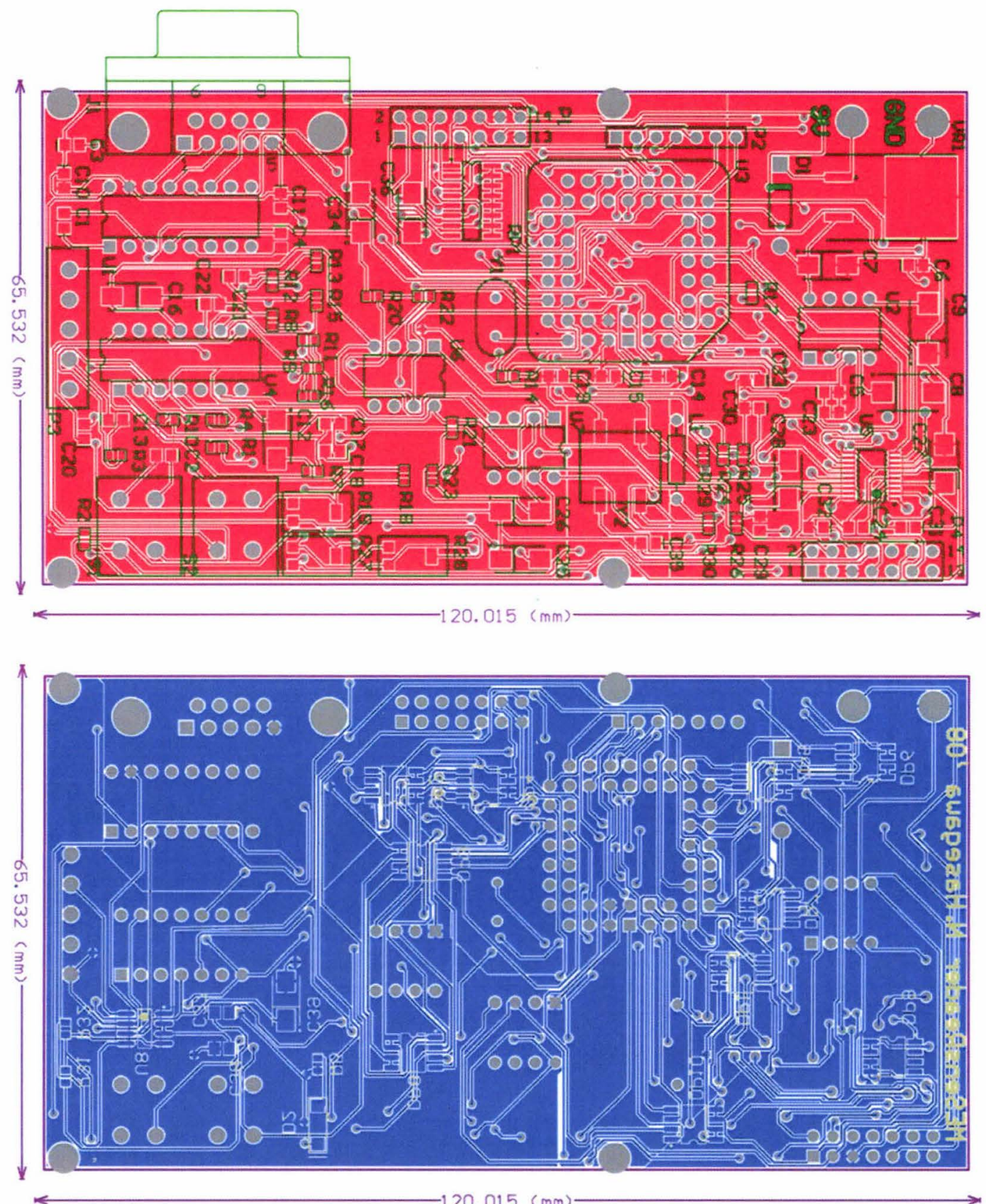


\section{Appendix C: Source Code}

\section{C source code for the AT89C51AC3 microcontroller:}

\section{1. main.c}

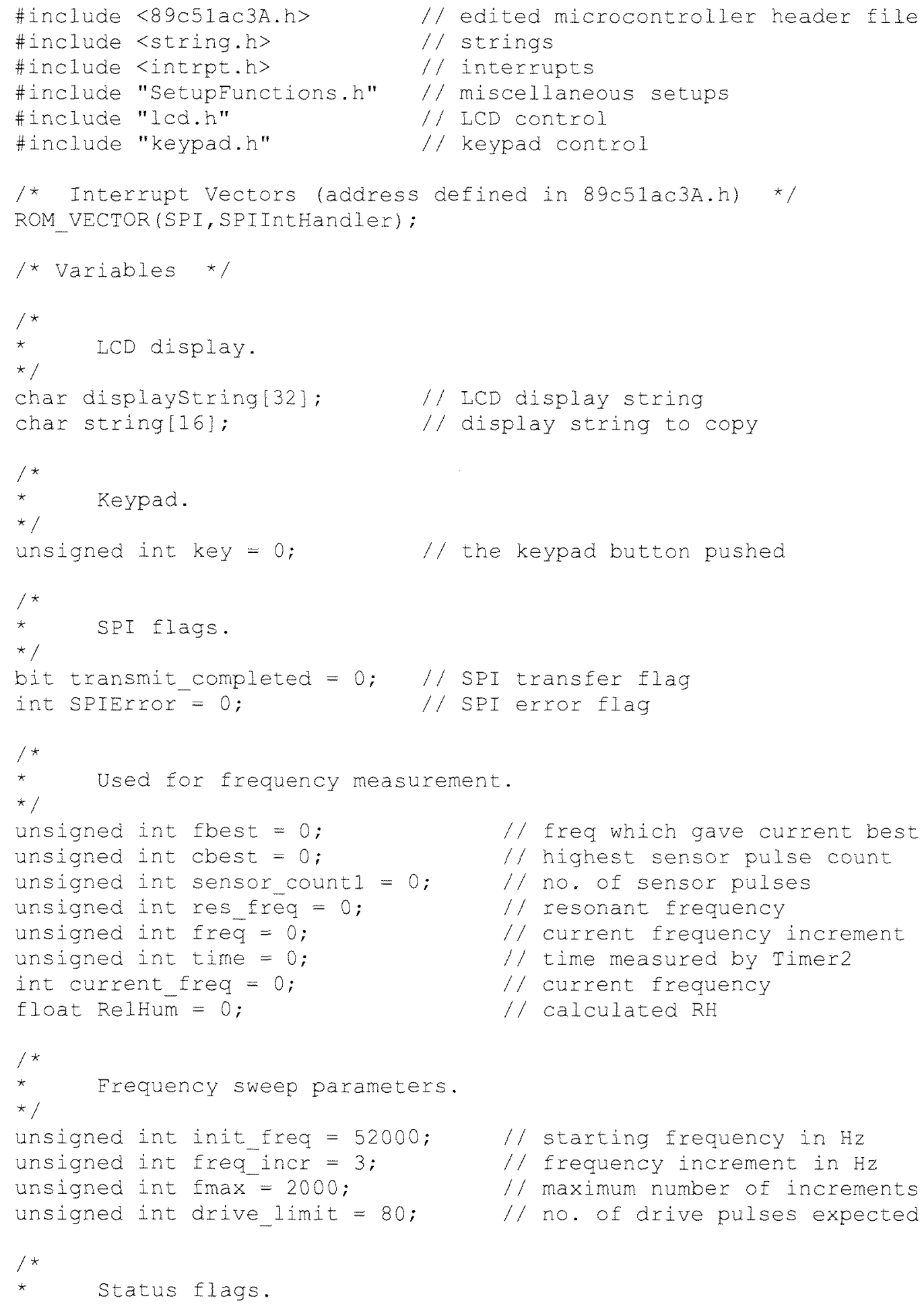




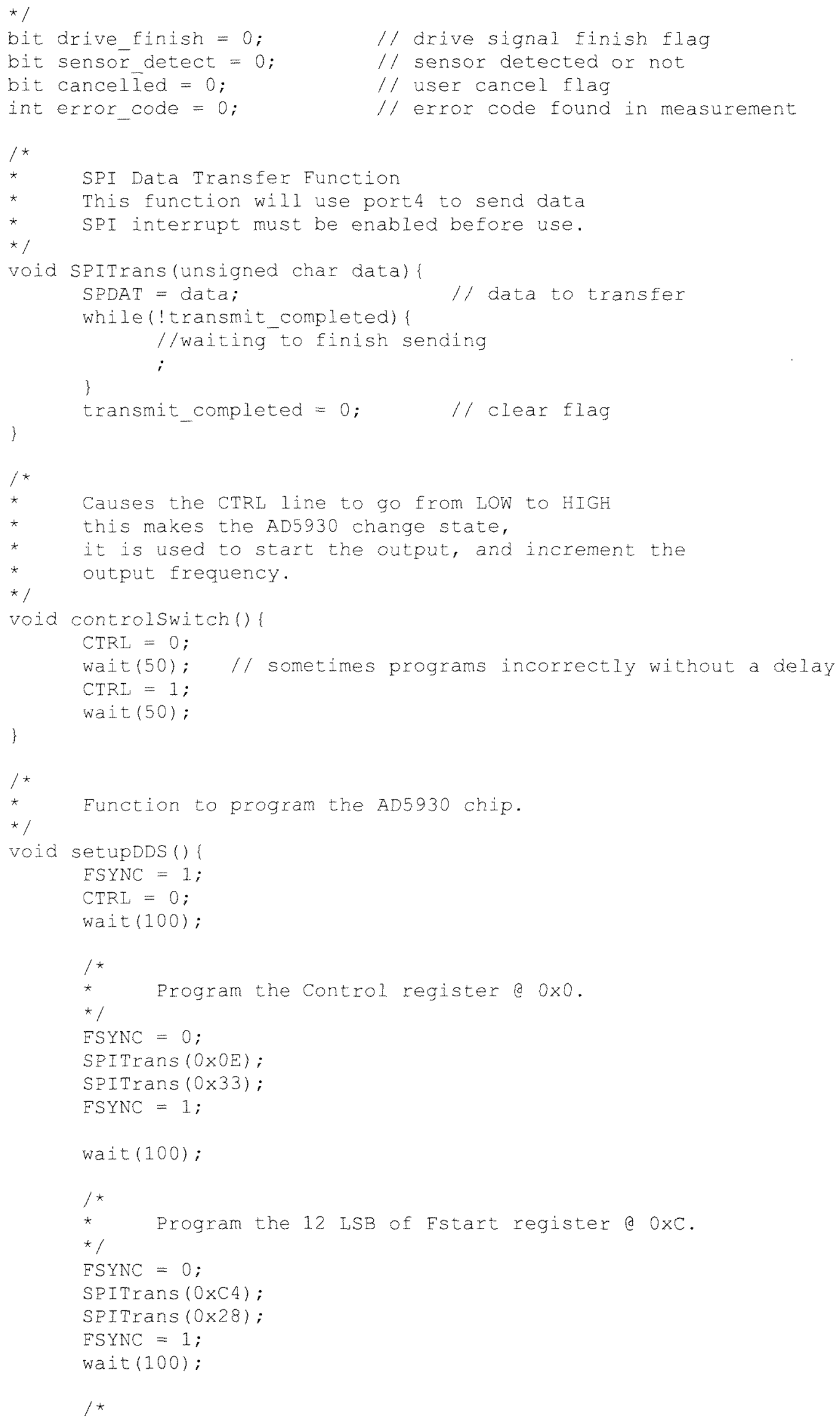




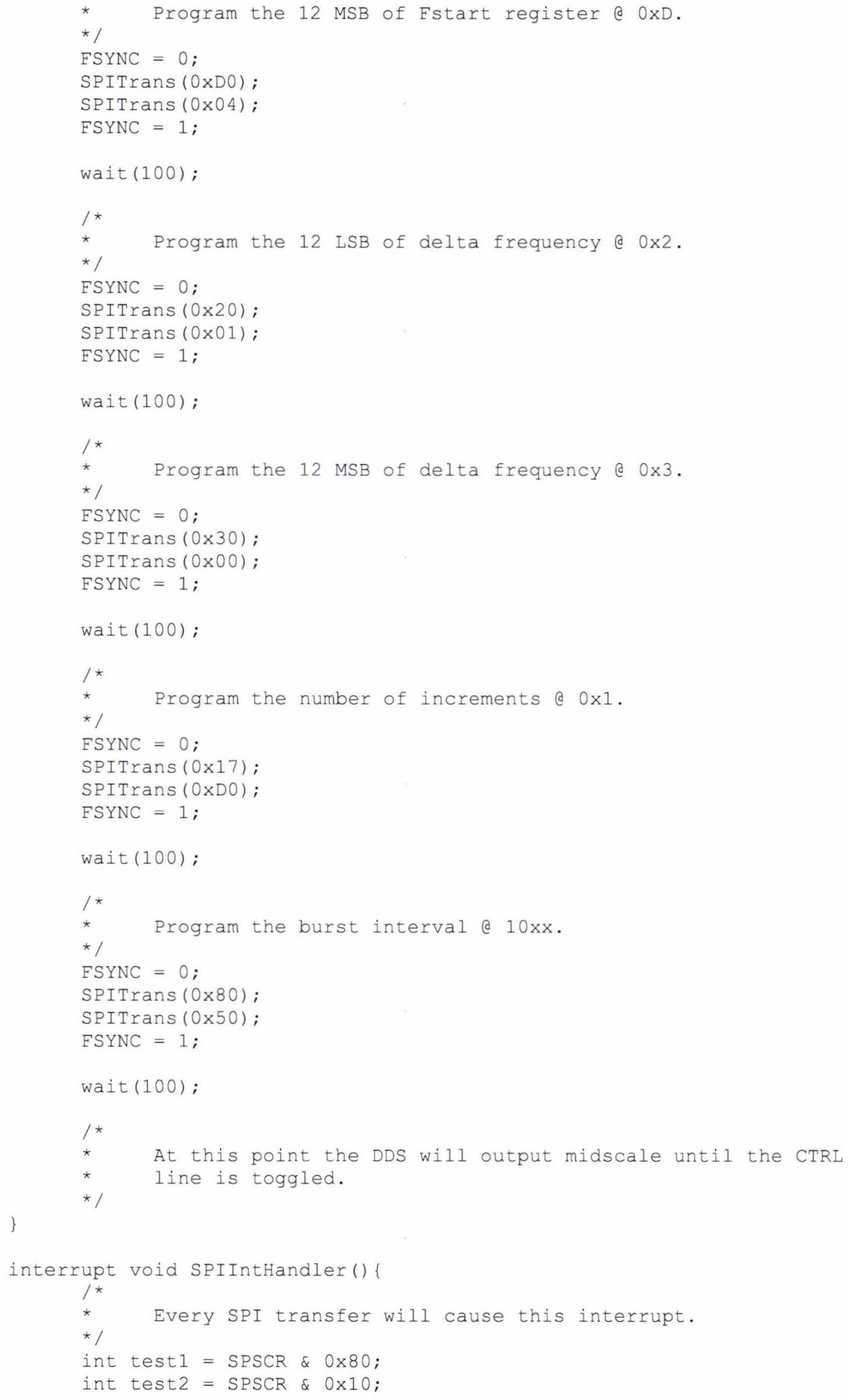




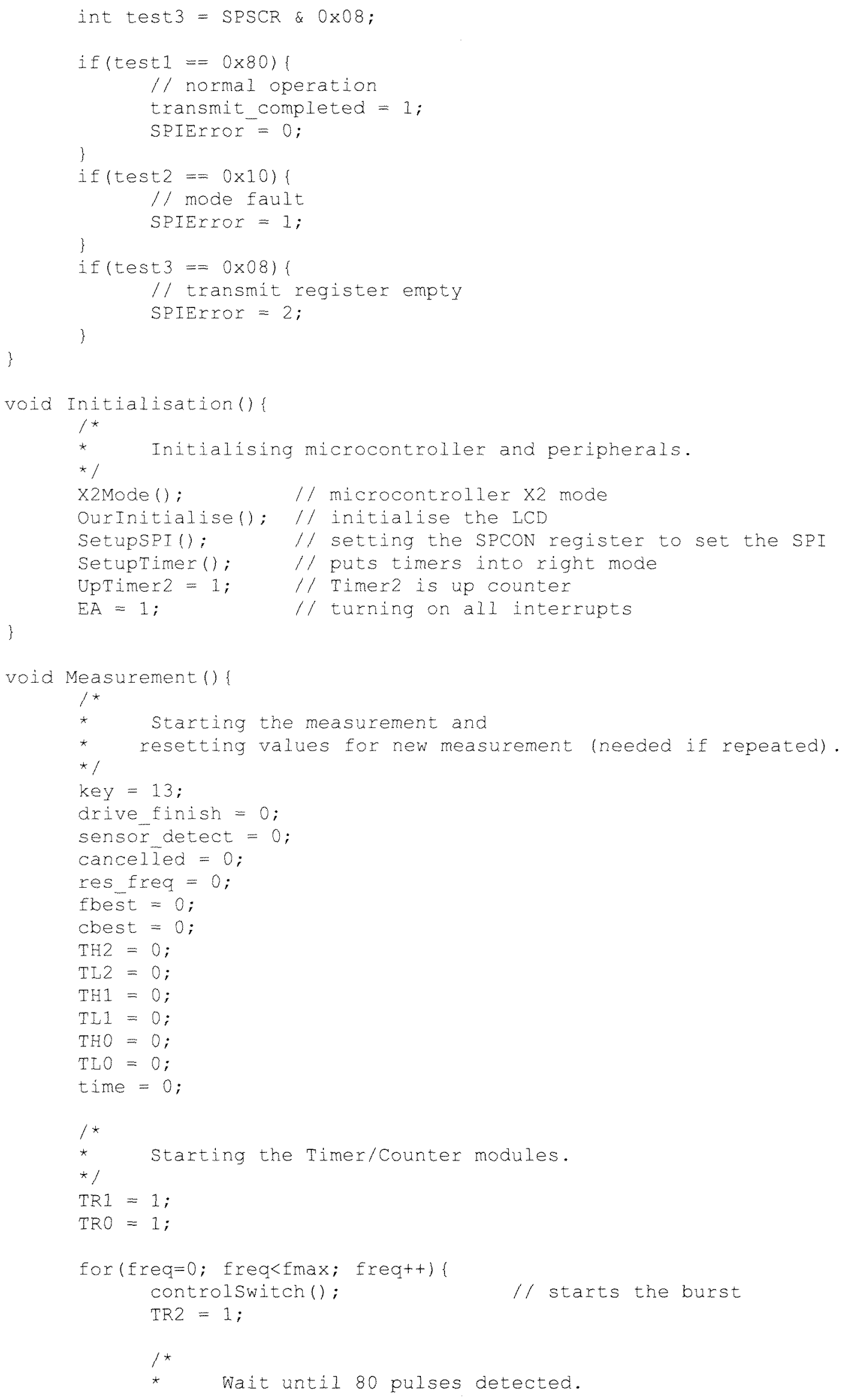




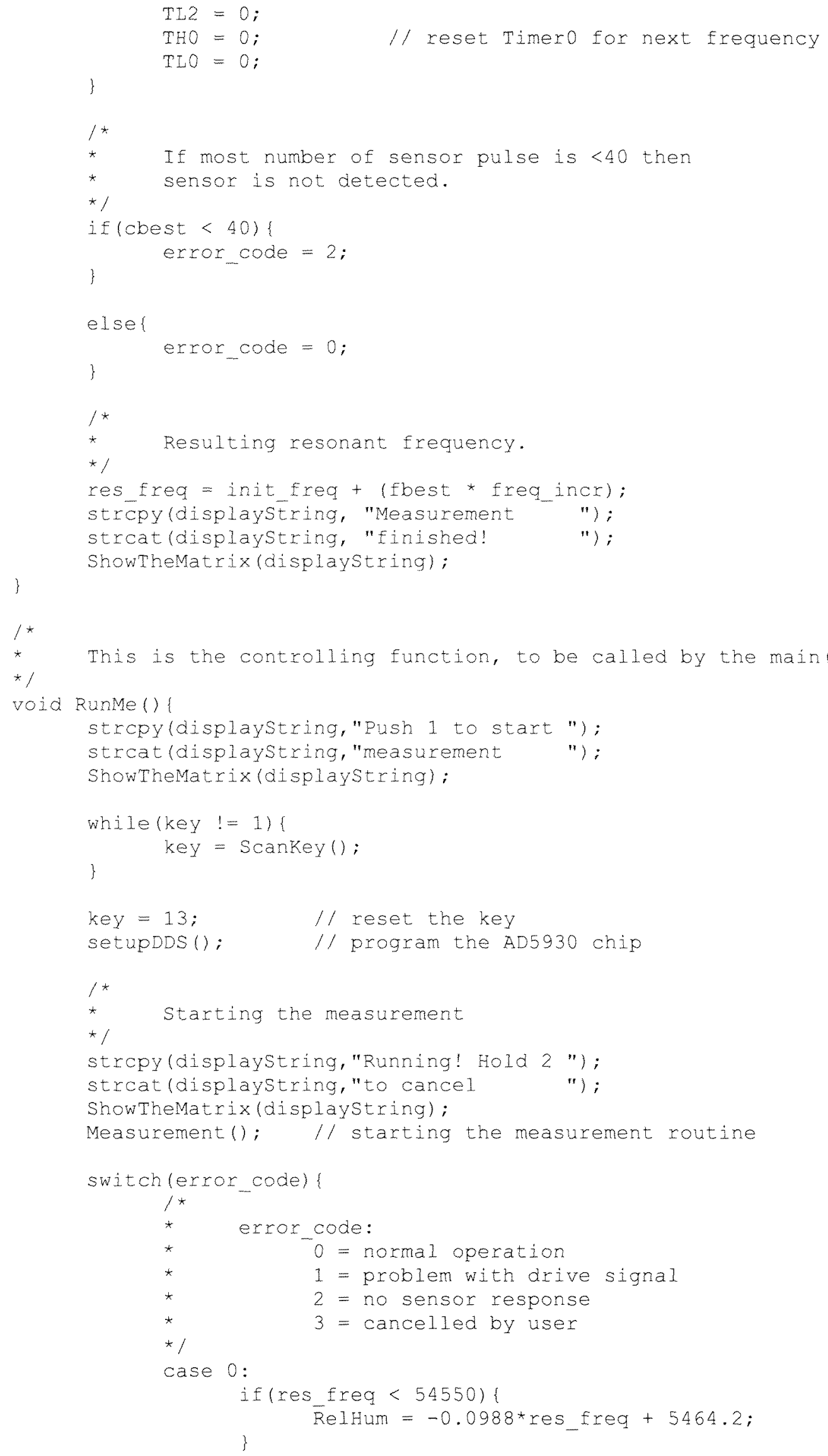




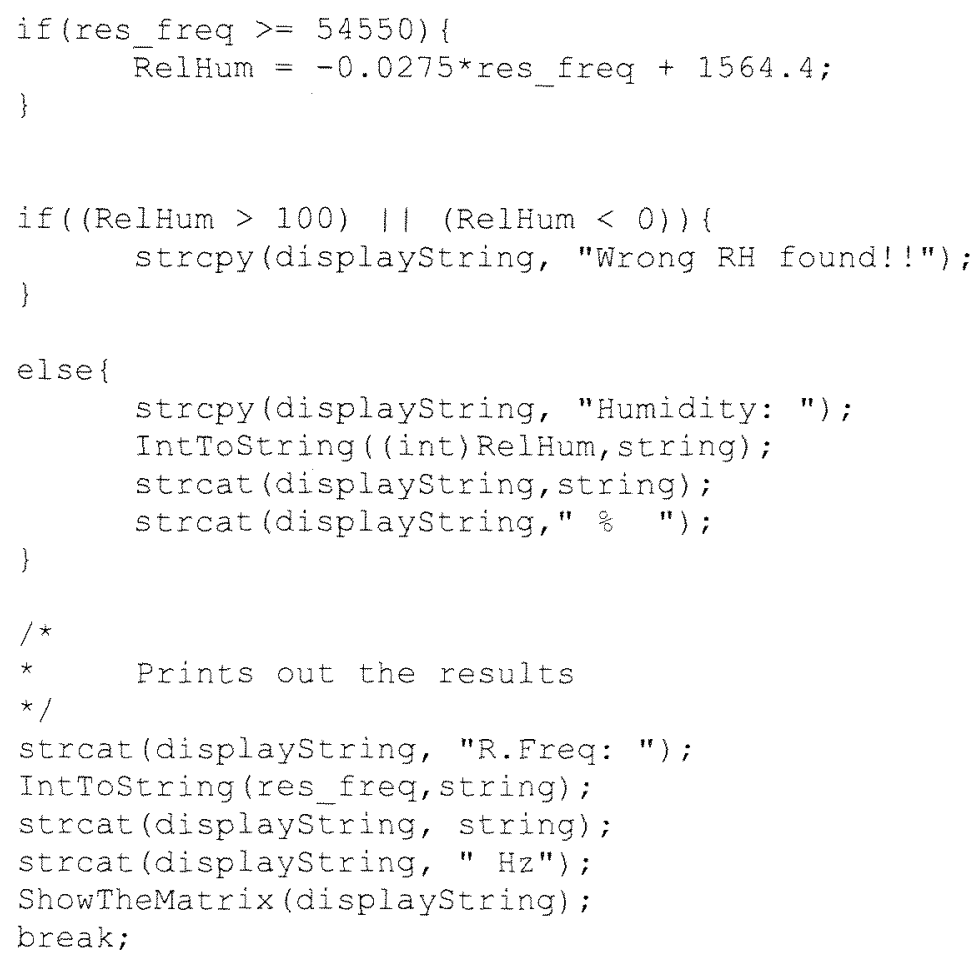




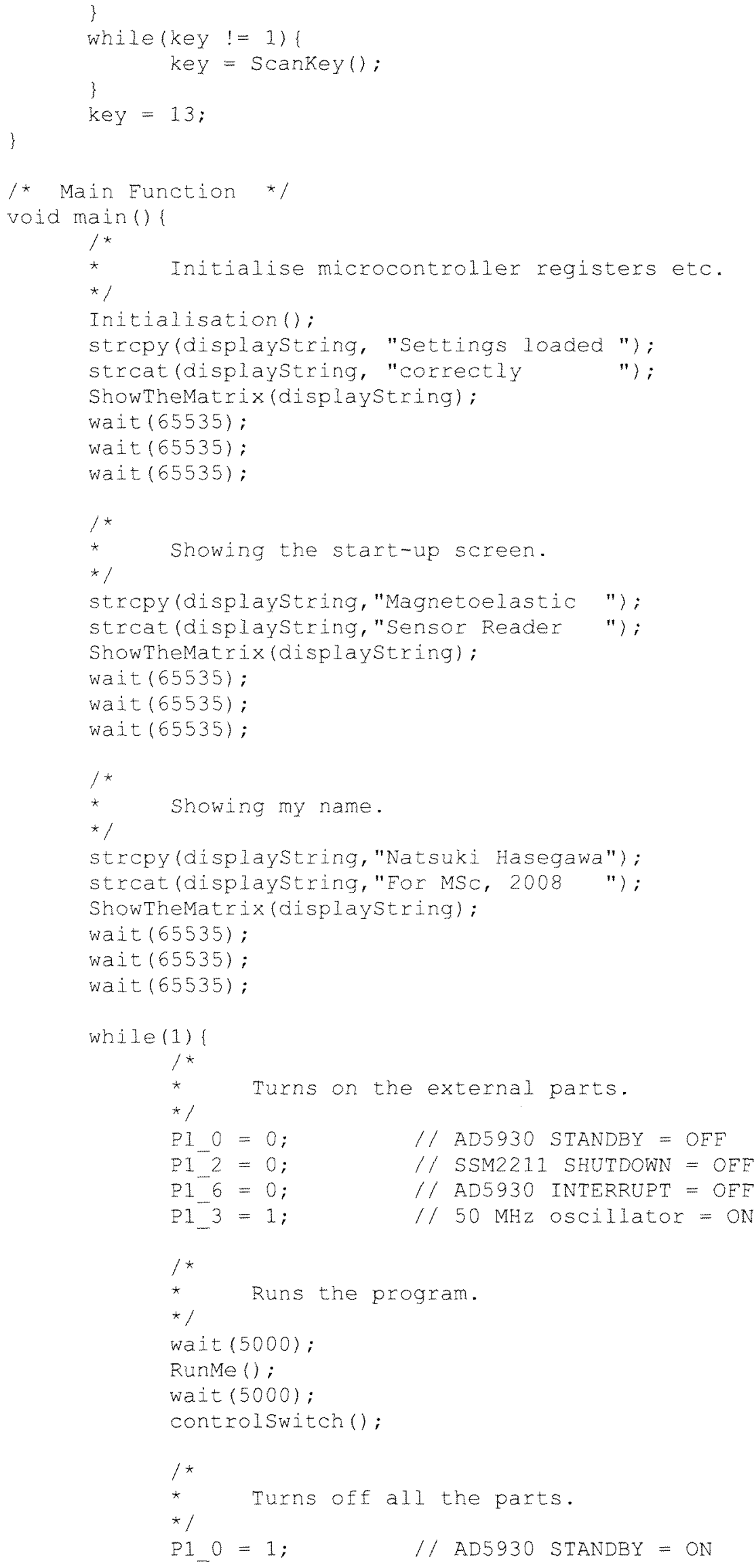




$$
\begin{array}{ll}
\mathrm{P1} 2=1 ; & / / \text { SSM2211 SHUTDOWN }=\text { ON } \\
\mathrm{P1} 6=1 ; & / / \text { AD5930 INTERRUPT }=\text { ON } \\
\mathrm{PI} 3=0 ; & / / 50 \mathrm{MHZ} \text { OSCI1Lto }=\text { OFE }
\end{array}
$$




\section{SetupFunctions.c}

\#include $\langle 89$ c51ac3A.h $\rangle$

\#include <string.h>

$1+$

sets up the SPI.

*

Void SetupseI()

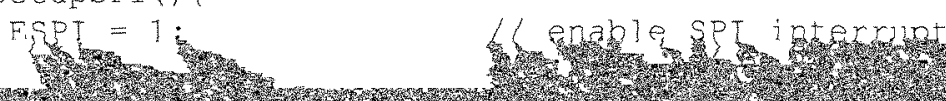

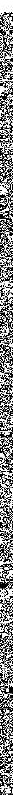




\section{LCD.c}

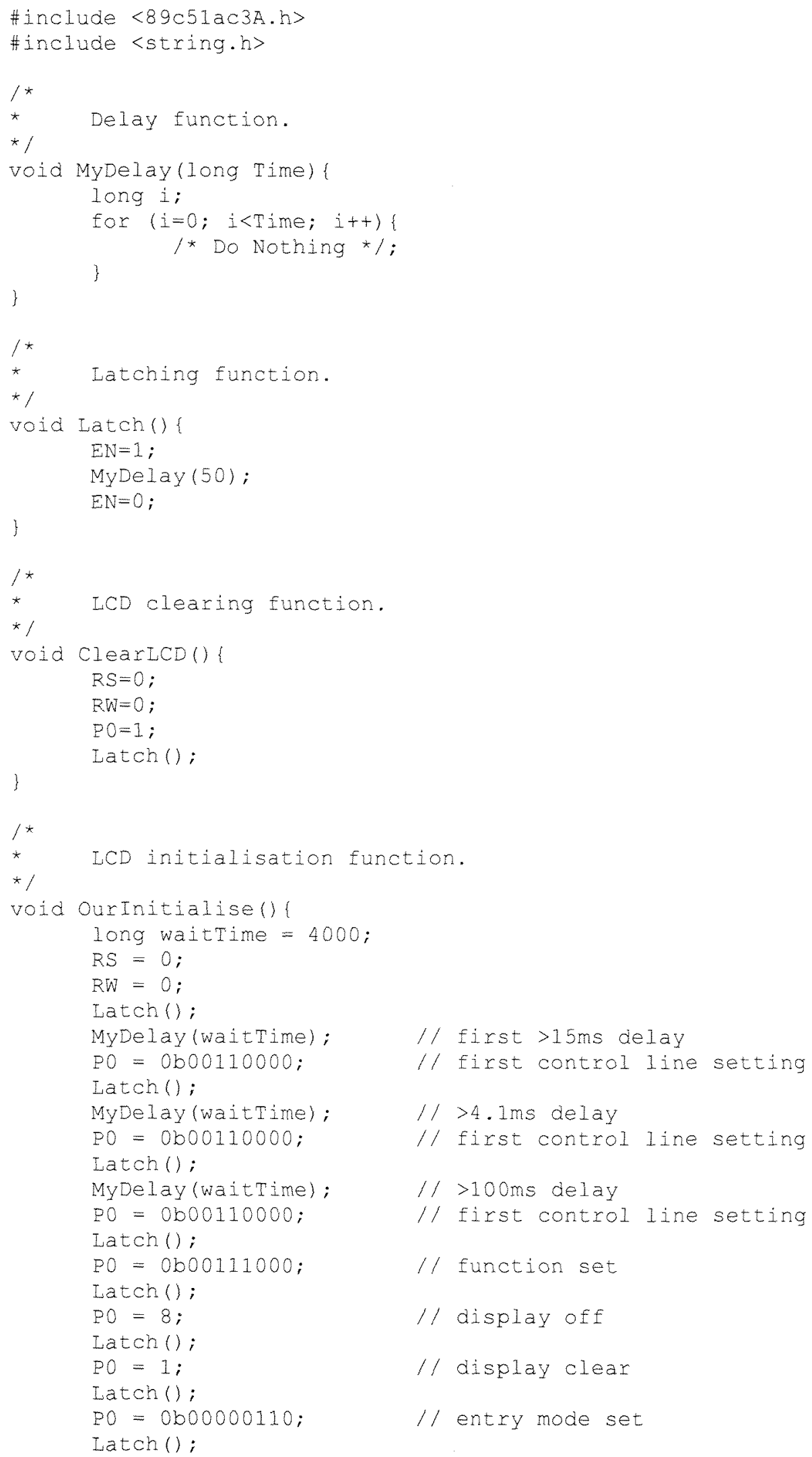




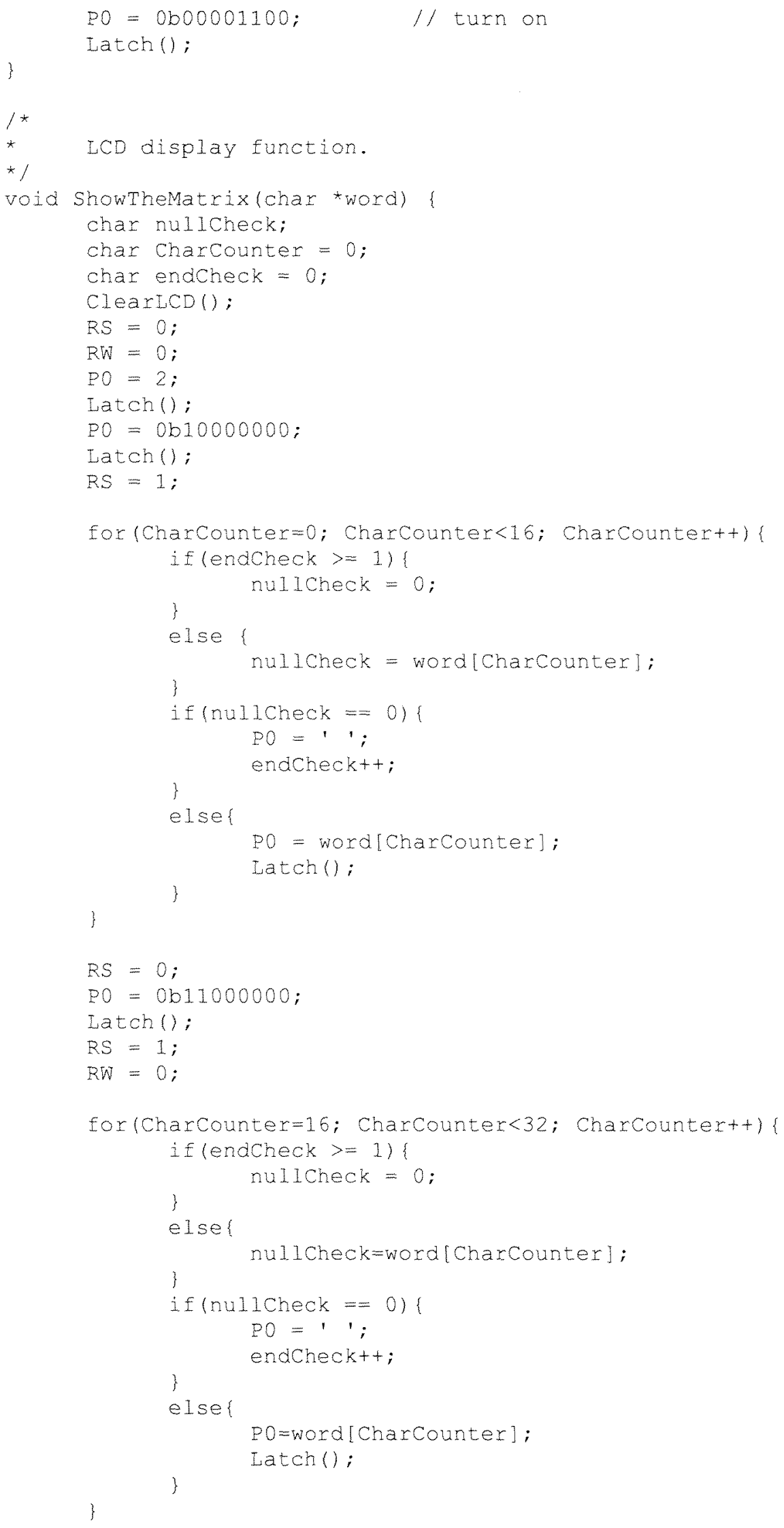




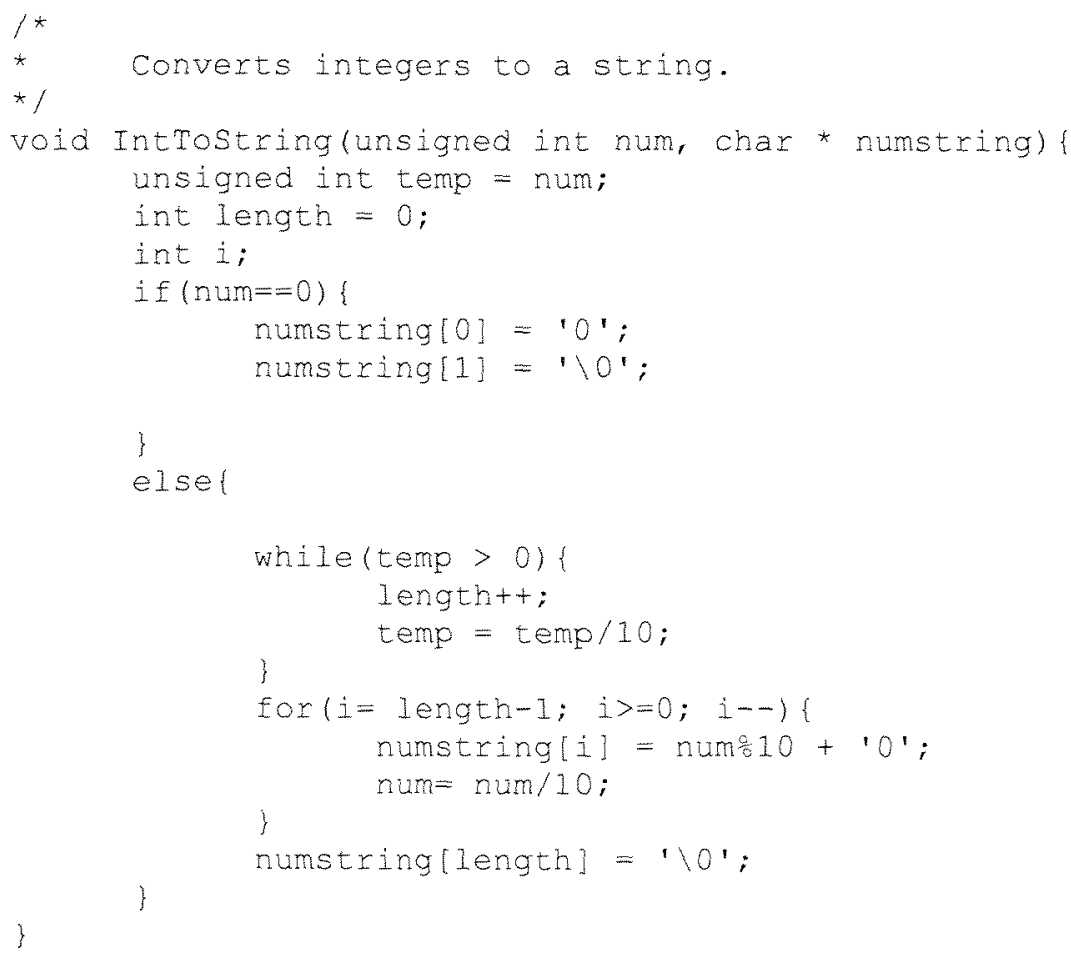




\section{4. keypad.c}

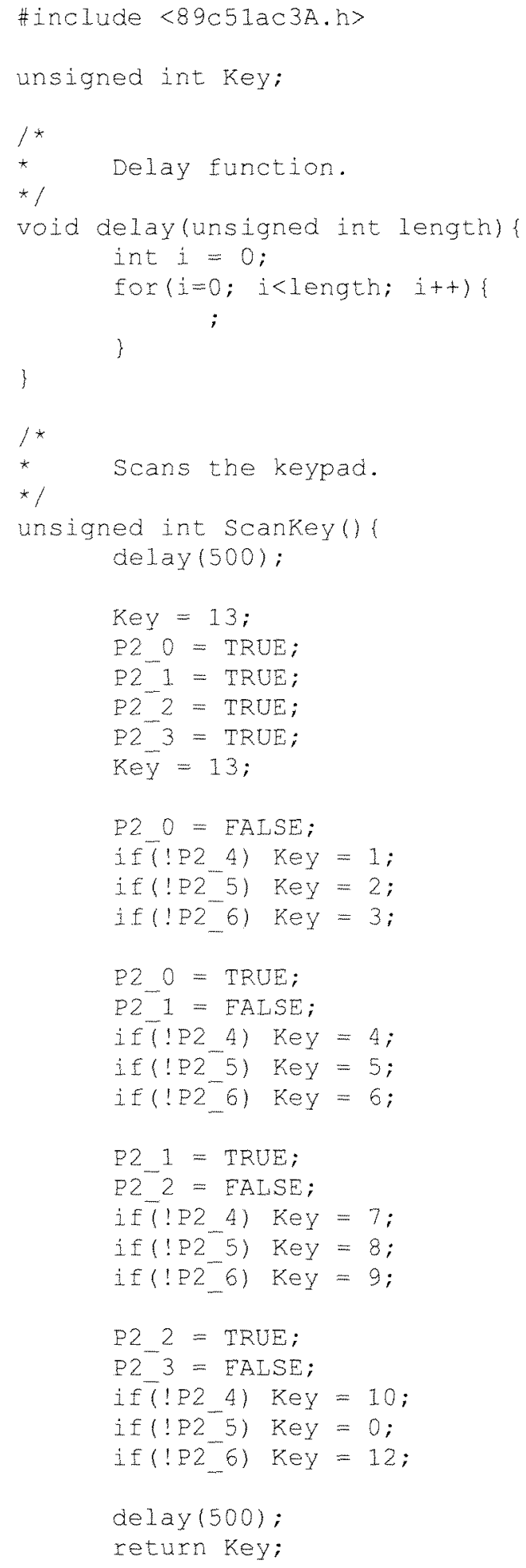




\section{89c51ac3A.h}

Header file for the AT89C51AC3 microcontroller used in the reader (edited from the original university version):

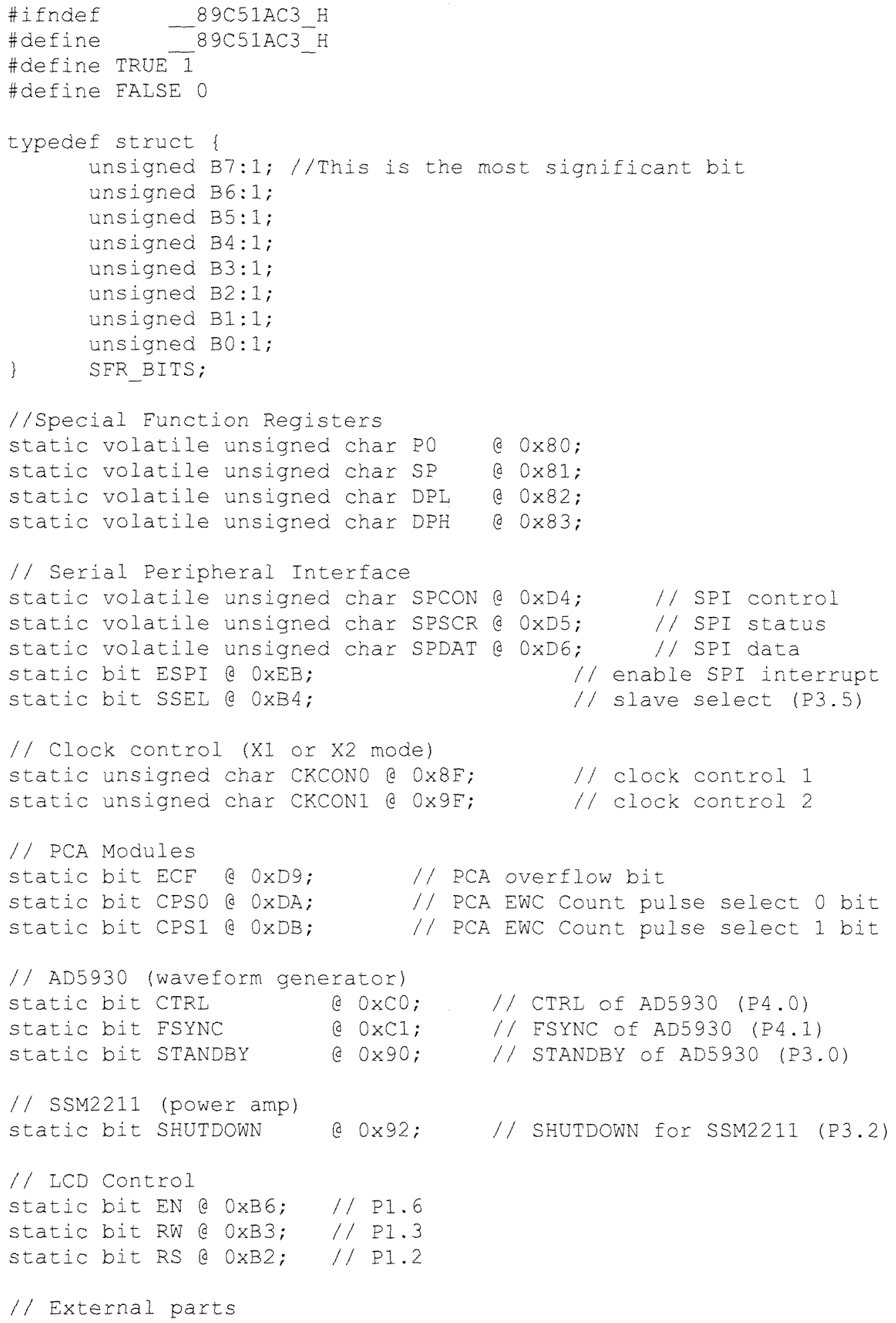




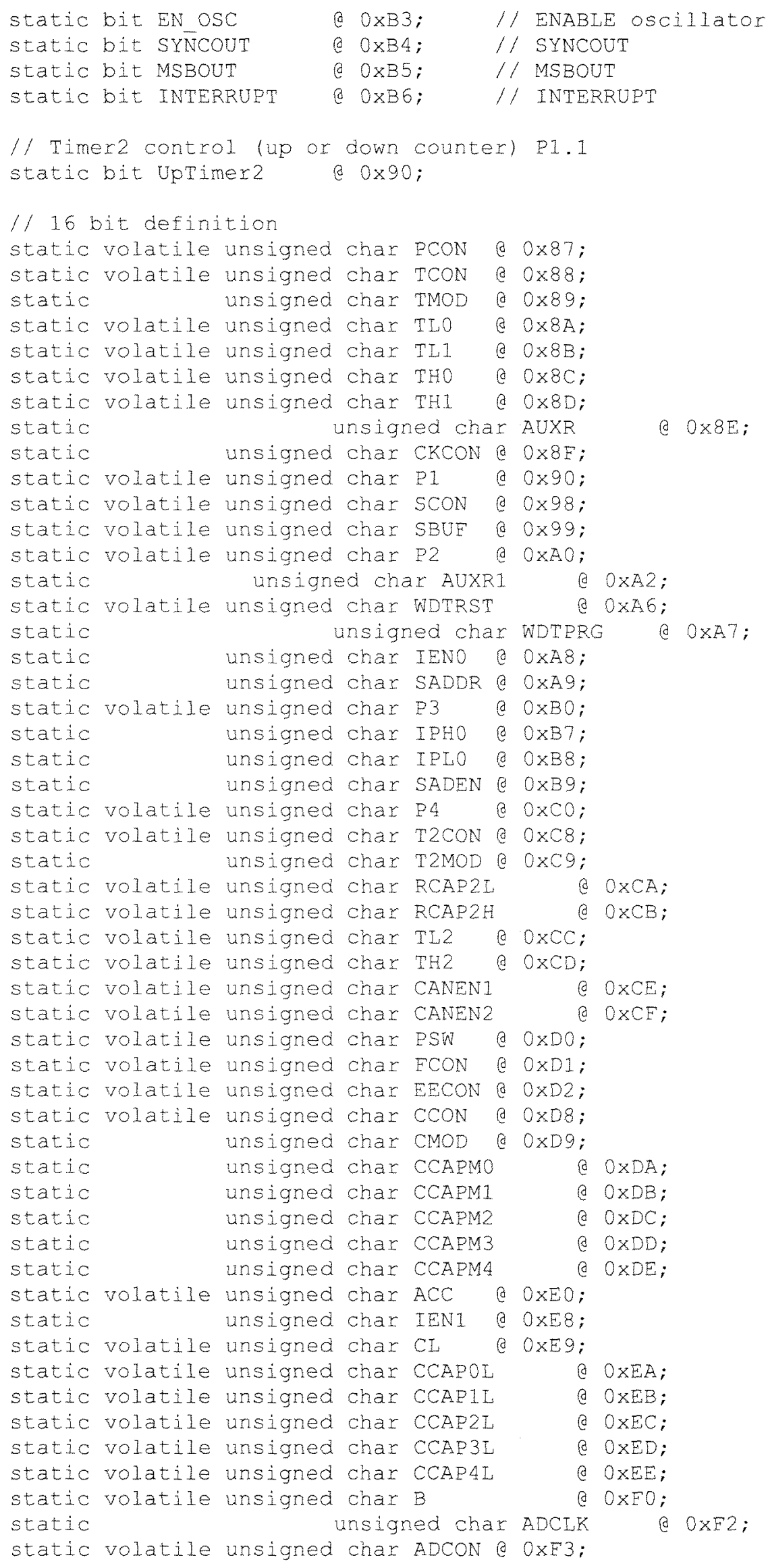




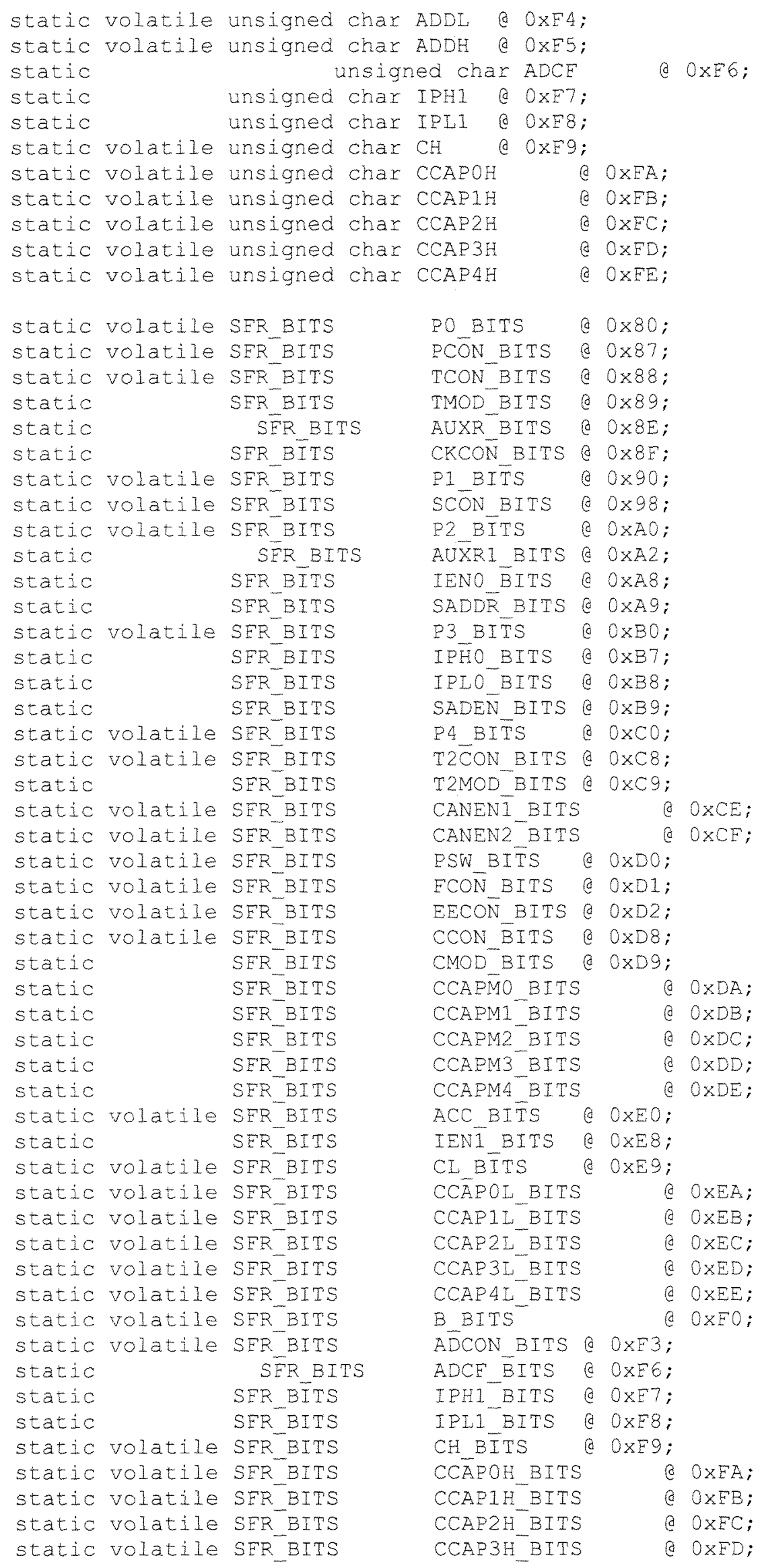




\begin{tabular}{|c|c|c|c|c|c|c|c|}
\hline \multicolumn{3}{|c|}{ static volatile SER BITS } & \multicolumn{3}{|c|}{ CCAPAH_BITS } & \multirow[t]{2}{*}{$\theta$} & \\
\hline $\begin{array}{l}/ / \text { Bit } \\
/ / \text { Port }\end{array}$ & $\begin{array}{l}\text { Addressable Loo } \\
0 \text { PO }\end{array}$ & ions & & & & & \\
\hline static & volatile bit & PO 0 & e & $0 \times 80$ & // VUW: & Was & Poo \\
\hline static & volatile bit & PO'1 & e & $0 \times 81$ & // VUW: & was & P01 \\
\hline static & volatile bit & $\mathrm{PO}_{-} 2$ & a & $0 \times 82 ;$ & // VUW: & Was & $\mathrm{P} 02$ \\
\hline static & volatile bit & $20^{-3} 3$ & a & $0 \times 83 ;$ & // VUW: & Was & P03 \\
\hline static & volatile bit & $\mathrm{PO}_{-}^{-4}$ & e & $0 \times 84 ;$ & // VUW: & Was & P04 \\
\hline static & volatile bit & $20^{-} 5$ & a & $0 \times 85$ & $/ /$ VUW: & Was & P05 \\
\hline static & volatile bit & $\mathrm{PO}^{-}-6$ & a & $0 \times 86$ & $1 /$ Vuw: & Was & P06 \\
\hline static & volatile bit & $20^{-} 7$ & e & $0 \times 87$ & // von: & Was & PO7 \\
\hline \multicolumn{8}{|c|}{ // Alternate Eunctions } \\
\hline static & volatile bit & ADO & a & $0 \times 80 ;$ & & & \\
\hline static & volatile bit & AD1 & a & $0 \times 81$ & & & \\
\hline static & volatile bit & AD2 & a & $0 \times 82$ & & & \\
\hline static & volatile bit & AD3 & a & $0 \times 83$ & & & \\
\hline static & volatile bit & ADA & e & $0 \times 84 ;$ & & & \\
\hline static & volatile bit & AD5 & @ & $0 \times 85$ & & & \\
\hline static & volatile bit & AD6 & e & $0 \times 86$ & & & \\
\hline static & volatile bit & $A D 7$ & e & $0 \times 87$ & & & \\
\hline \multicolumn{8}{|c|}{ //Timer Control TCON } \\
\hline static & $b i t$ & ITO & B & $0 \times 88$ & & & \\
\hline static & volatile bit & IEO & a & $0 \times 89 ;$ & & & \\
\hline static & bit & IT1 & e & $0 \times 8 \mathrm{~A}$ & & & \\
\hline static & volatile bit & IEI & e & $0 \times 8 B$ & & & \\
\hline static & bit & TRO & a & $0 \times 8 \mathrm{C}$ & & & \\
\hline static & volatile bit & TEO & (a) & $0 \times 80$ & & & \\
\hline static & bit & TRI & a & $0 \times 8 \mathrm{E}$ & & & \\
\hline static & volatile bit & $T E 1$ & de & $0 \times 8 E$ & & & \\
\hline \multicolumn{8}{|c|}{$/ /$ Port $1 \mathrm{P} 1$} \\
\hline static & volatile bit & P1 0 & a & $0 \times 90$ & // VOW: & Was & P10 \\
\hline static & volatile bit & P1]1 & e & $0 \times 91$ & $/ /$ VUW: & Was & P11 \\
\hline static & volatile bit & $\mathrm{P} 12$ & d & $0 \times 92 ;$ & // vuw: & Was & $\mathrm{P} 12$ \\
\hline static & volatile bit & 213 & a & $0 \times 93$ & // VUW: & Was & P13 \\
\hline static & volatile bit & $\mathrm{P} 1-4$ & a & $0 \times 94$ & // VUW: & Was & P14 \\
\hline static & volatile bit & $\mathrm{P} 1-5$ & e & $0 \times 95$ & $1 /$ Vun: & Was & P15 \\
\hline static & volatile bit & P1_6 & d & $0 \times 96$ & $/ / \mathrm{VUW}:$ & Was & P16 \\
\hline static & volatile bit & $\mathrm{PI}_{-}^{-7}$ & a & $0 \times 97$ & $1 /$ VUw: & Was & $\mathrm{P} 17$ \\
\hline \multicolumn{8}{|c|}{ // Alternate functions } \\
\hline static & volatile bit & T2 & b & $0 \times 90$ & & & \\
\hline static & volatile bit & T2EX & a & $0 \times 91 ;$ & & & \\
\hline static & volatile bit & ECI & e & $0 \times 92 ;$ & & & \\
\hline statio & volatile bit & $\mathrm{CEXO}$ & a & $0 \times 93$ & & & \\
\hline static & volatile bit & CEX1 & e & $0 \times 94 ;$ & & & \\
\hline static & volatile bit & CEX2 & b & $0 \times 95$ & & & \\
\hline static & volatile bit & $\mathrm{CEX} 3$ & e & $0 \times 96$ & & & \\
\hline static & volatile bit & CEX4 & a & $0 \times 97 ;$ & & & \\
\hline \multicolumn{8}{|c|}{ //Serial Control SCON } \\
\hline static & volatile bit & RI & a & $0 \times 98 ;$ & & & \\
\hline static & volatile bit & $T I$ & d & 0x99; & & & \\
\hline static & volatile bit & RB8 & e & $0 \times 9 A$ & & & \\
\hline static & bit & TB8 & e & $0 \times 9 B$ & & & \\
\hline static & bit & REN & e & $0 \times 9 \mathrm{C}$ & & & \\
\hline static & bit & SM2 & a & $0 \times 90$ & & & \\
\hline static & bit & SM1 & a & $0 \times 9 E$ & & & \\
\hline
\end{tabular}




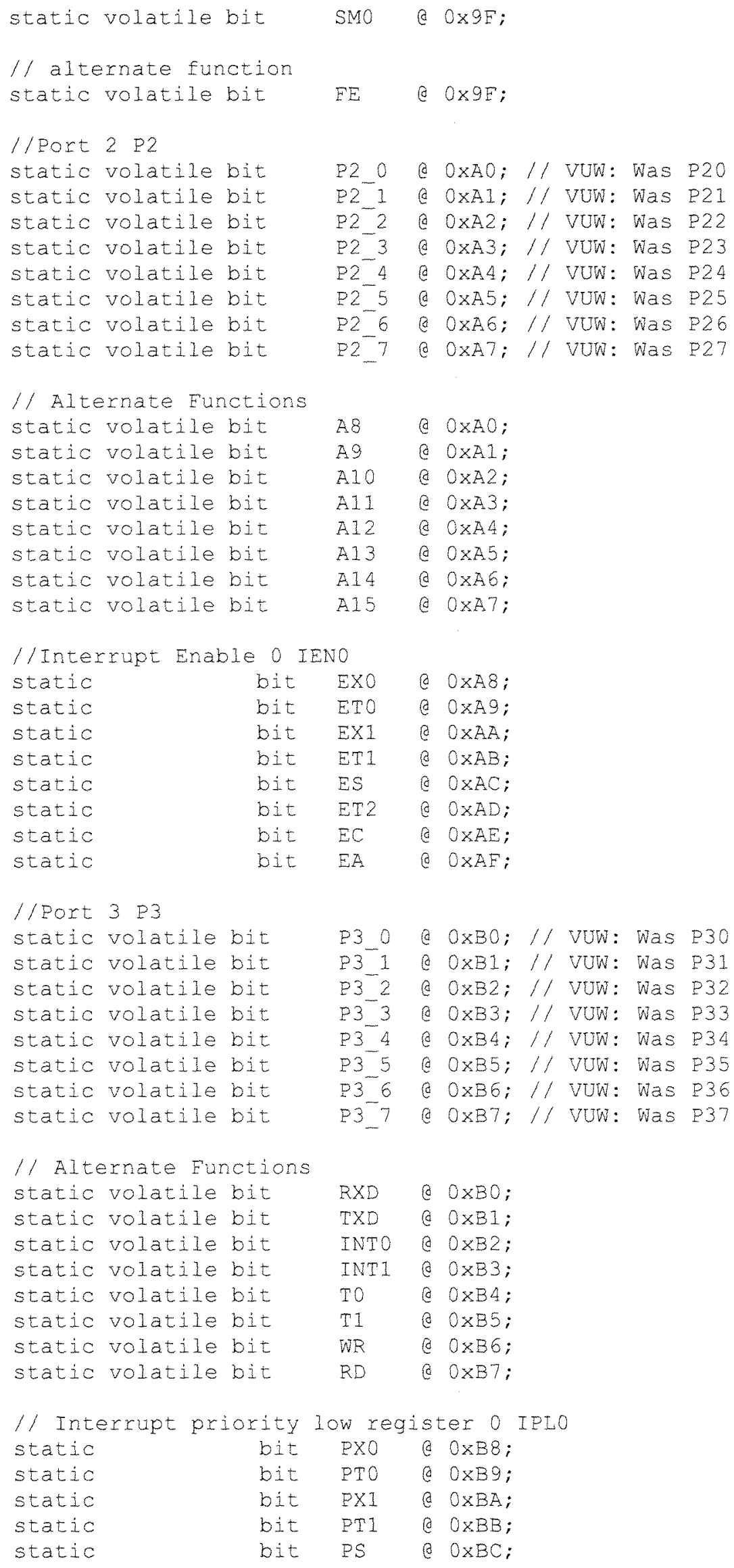




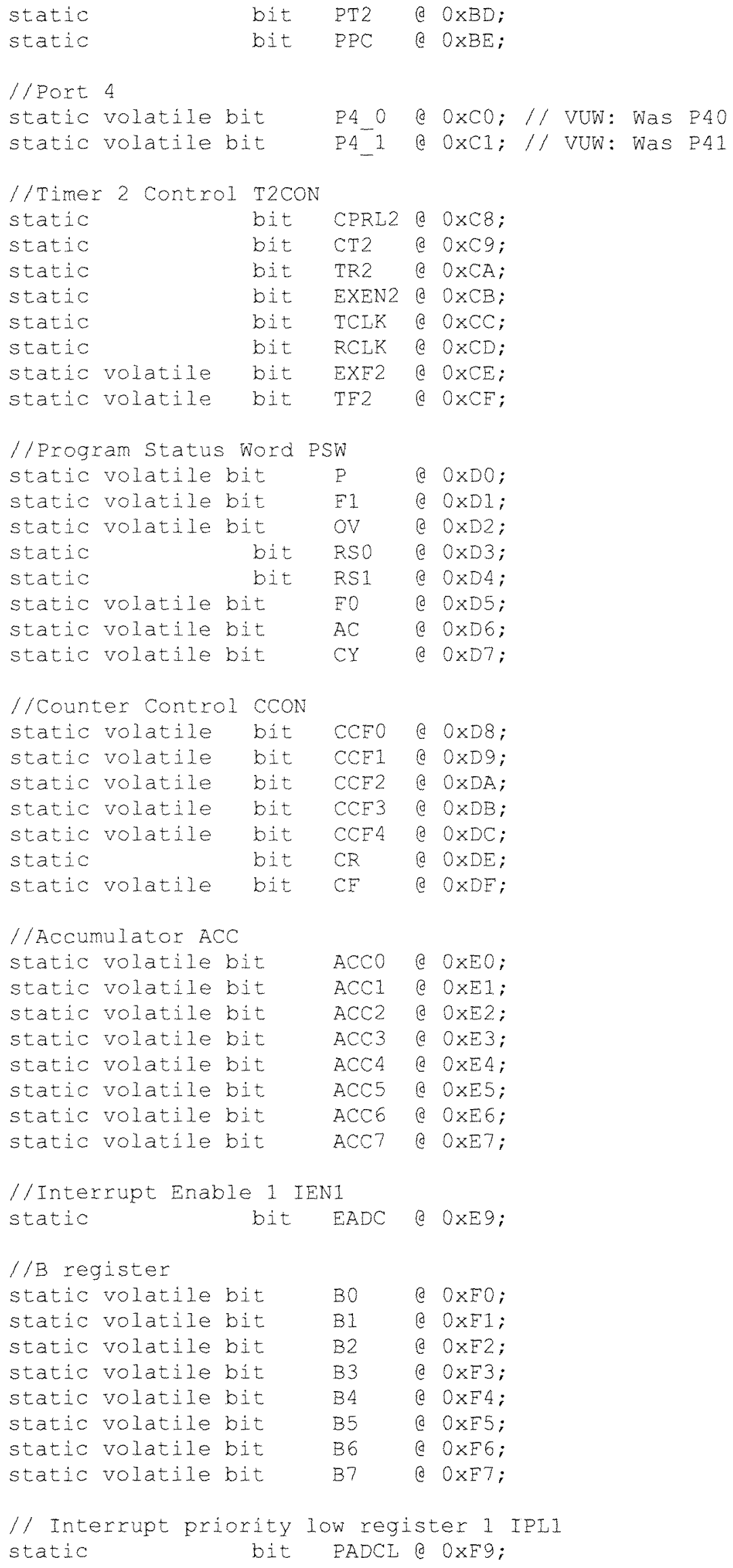


/ Interrupt vectors

\#define RESET 0

FXTINTO

\#define TIMERO

\#define EXTINT1

\#define TIMERI

\#define SERIAI

\#define TIMER2

\#define PCA Ox33

\#define ADC Ox43

\#define SPI $0 \times 53$

\#endif
$0 \times 03$

$0 \times 0 B$

$0 \times 13$

$0 \times 1 \mathrm{~B}$

$0 \times 23$

$0 \times 2 B$ 


\section{References}

[1] Herzer, G., "Magnetic materials for electronic article surveillance", Journal of Magnetism and Magnetic Materials, 254-255, p598-602, 2003.

[2] Zeng, K., Paulose, M., Ong, K. G., Grimes, C. A., "Frequency-domain characterization of magnetoelastic sensors: a microcontroller-based instrument for spectrum analysis using a threshold-crossing counting technique", Sensors and Actuators A, 121, p66-71, 2005.

[3] New Zealand Herald, "NZ Herald: Leaky Buildings", $<$ http://www.nzherald.co.nz/feature/index.cfm?c_id $=562>$, accessed 29/8/2008.

[4] Consumer New Zealand, "ConsumerBuild: Leaky Buildings", $<$ http://www.consumerbuild.org.nz/publish/leaky.php>

[5] Smith, G., “Magnetoelastic Materials for Environmental Monitoring: TECH40I Research Project', Unpublished, Victoria University of Wellington, 2007.

[6] Siemens, "Siemens $R \& I$ - To catch a thief", $<$ http://w4.siemens.de/Ful/en/archiv/zeitschrift/heft1_99/artikello/index.html>, accessed 18/2/2008.

[7] HowStuffWorks.com, "How Anti-shoplifting Devices Work", $<$ http://electronics.howstuffworks.com/anti-shoplifting-device.htm $>$, accessed $18 / 2 / 2008$.

[8] Herzer, G., "Method of Annealing Amorphous Ribbons and Marker for Electronic Article Surveillance", United States Patent 6,011,475, January 4, 2000 . 
[9] Anderson, III et al, "Surveillance System Having Magnetomechanical Marker", United States Patent 4,510,489, April 9, 1985.

[10] Telford, M., "The case for bulk metallic glass", Materials Today, p36-43, March 2004.

[11] Livingston, J. D., "Driving Force: The Natural Magic of Magnets", Harvard University Press, 1996.

[12] Metglas Inc., "Metglas 2826MB Alloy" $<$ http://www.metglass.com/products/page5_1 2 $7 . \mathrm{htm}>$, accessed 5/12/2007.

[13] Gadonniex, D. M., Hansen, N., "Electronic Article Surveillance Marker", United States Patent Applications Publication, US 2008/0030339 A1, February 7, 2008.

[14] Livingston, J. D., Morris, W. G., "Magnetic Domains in Amorphous Metal ribbons", Journal of Applied Physics 57 (1), p3555-3559, April 1985.

[15] Halliday, D., Wesnick, R., Walker, J., "Fundamentals of physics 6th edition", John Wiley \& Sons Inc., 2001.

[16] LiquidMetal Technologies, "LiquidMetal Technologies Website", $<$ http://www.liquidmetal.com>, accessed 10/7/2008.

[17] Myers, H. P., "Introduction to Solid State Physics", Taylor and Francis, 1990.

[18] Moskovitz, B. M., "Hitchhiker's Guide to Magnetism", University of Minnesota Institute for Rock Magnetism, 1991.

[19] Bickford, L. R., "Magnetism during the IEEE's First One Hundred Years (1884-1984)", IEEE Transactions on Magnetics, Vol. Mag-21, No. 1, p1-8, January 1985. 
[20] Chikazumi, S., "Physics of Magnetism", John Wiley \& Sons Inc., 1964.

[21] Hernando, A., Vazquez, M., Barandiaran, J. M., "Metallic Glasses and Sensing Applications", Journal of Physics E: Scientific Instruments 21, p1129$1139,1988$.

[22] Georgia State University, "HyperPhysics: Magnetostriction", $<$ http://hyperphysics.phy-astr.gsu.edu/hbase/solids/magstrict.html $>$, accessed $5 / 5 / 08$

[23] Bozorth, R. M., "Ferromagnetism”, Princeton, N.J. Van Nostrand, 1961.

[24] Mitchell, E. E., De Moyer, R., Vranish, J., "A New Metglas Sensor", IEEE Transactions on Industrial Electronics, Vol. IE-33, No. 2, p166-170 May 1986.

[25] Georgia State University, "HyperPhysics: Young's Modulus", $<$ http:/hyperphysics.phy-astr.gsu.edu/hbase/permot3.html>, accessed 20/8/2008.

[26] Grimes, C. A., Mungle, C. S., Zeng, K., Jain, M. K., Dreschel, W. R., Paulose, M., Ong, K. G., “Wireless Magnetoelastic Resonance Sensors: A Critical Review", Sensors 2002, p294-313, 2002.

[27] Livingston, J. D., "Magnetomechanical Properties of Amorphous Metals", physica status solidi (a) 70, p591-596, 1982.

[28] Squire, P. T., "Phenomenological model for magnetization, magnetostriction and $\triangle E$ effect in field-annealed amorphous ribbons", Journal of Magnetism and Magnetic Materials, Vol. 87, 1990.

[29] Grimes, C. A., Kouzoudis, D., "Remote query measurement of pressure, fluid-flow velocity, and humidity using magnetoelastic thick film sensors", Sensors and Actuators 84, p205-212, 2000. 
[30] Guntupalli, R., Hua. J., Lakshmanan R. S., Huang, T. S., Barbate, J. M., Chin, B. A., "A magnetoelastic resonance biosensor immobilized with polyclonal antibody for the detection of Salmonella typhimurium", Biosensors and Bioelectronics 22, p1474-1479, 2007.

[31] Ong, K. G., Leland, J. M., Zenga K., Barrett, G., Zourob, M., Grimes, C. A., "A rapid highly-sensitive endotoxin detection system", Biosensors and Bioelectronics 21, p2270-2274, 2006.

[32] Pang, P., Gao, X., Xiao, X., Yang, W., Cai, Q., Yao, S., “ $A$ Wireless $p H$ Sensor Using Magnetoelasticity for Measurement of Body Fluid Acidity", Analytical Sciences, Vol. 23, p463-467, April 2007.

[33] Wan, J., Shu, H., Huang, S., Fiebor, B., Chen, I., Petrenko, V. A., Chin, B. A., "Phage-Based Magnetoelastic Wireless Biosensors for Detecting Bacillus Anthracis Spores", IEEE Sensors Journal, Vol. 7, No. 3, p470-477, March 2007.

[34] Atmel Corp., "AT89C51AC3 datasheet", $<$ http://atmel.com/dyn/resources/prod_documents/doc4383.pdf $>$, accessed 18/8/2008.

[35] HI-TECH Software, "HI-TIDE 2", $<$ http://www.htsoft.com/products/hitide/hitide2.php>, accessed 23/8/2008.

[36] Atmel Corp., "Atmel FLIP",

$<$ http://www.atmel.com/dyn/products/tools_card.asp?tool_id=3886>, accessed 23/8/2008

[37] Analog Devices Inc., "AD9852 datasheet", $<$ hitp://www.analog.com/static/imported-files/data_sheets/AD9852.pdf>, accessed 23/8/2008. 
[38] Analog Devices Inc., "AD5930 datasheet", <http://www.analog.com/static/imported-files/data_sheets/AD5930.pdf>, accessed 23/8/2008.

[39] Analog Devices Inc., "AD5932 datasheet", <http://www.analog.com/static/imported-files/data_sheets/AD5932.pdf>, accessed 23/8/2008.

[40] Analog Devices Inc., "AD5930 evaluation board datasheet", < http://www.analog.com/static/imported-files/eval_boards/ /47694415155145AD5930EB_0.pdf>, accessed 23/8/2008.

[41] Epson Toyocom Corp., "SG-8002JC/JA series datasheet", $<$ http://ndap3-net.ebz.epson.co.jp/w/www/PDFS/epdoc_qd.nsf/ $766 f 09$ cc 8 f 2 fef704925706f002872df/e707968018e2c0a14925707c003d5f08/ /SFILE/SG-8002JC_JA_E07X.pdf $>$, accessed 23/8/2008.

[42] Analog Devices Inc., "Analog Devices DDS FAQs", $<$ http://www.analog.com/static/imported-files/faqs/DDS_FAQ.pdf $>$, accessed $23 / 10 / 2007$.

[43] Zeng, K., Ong, K. G., Mungle, C., Grimes, C. A., "Time Domain Characterization of Oscillating Sensors: Application of Frequency Counting to Resonance Frequency Determination", Review of Scientific Instruments, Vol. 73, No.12, p4375-4381, 2002.

[44] National Semiconductor Corp., "LM384 datasheet", $<$ http://www.national.com/ds/LM/LM384.pdf>, accessed 23/8/2008.

[45] Analog Devices Inc., "SSM2211 datasheet", $<$ http://www.analog.com/static/imported-files/data_sheets/SSM2211.pdf>, accessed 23/8/2008. 
[46] Texas Instruments Inc., "OPA227/OPA228 datasheet", $<$ http://focus.ti.com/lit/ds/symlink/opa228.pdf>, accessed 23/8/2008.

[47] National Semiconductor Corp., "LM111/LM211/LM311 datasheet", $<$ http://www.national.com/ds.cgi/LM/LM111.pdf>, accessed 23/8/2008.

[48] STMicroelectronics, "L4940xx datasheet", $<$ http://www.st.com/stonline/products/literature/ds/2141.pdf $>$, accessed 23/8/2008.

[49] Linear Technology Corp., "LT1054/LT1054L datasheet", $<$ http://www.linear.com/pc/downloadDocument.do?navId=H0,Cl,C1003,Cl0 39,C1014,P1258,D2309>, accessed 23/8/2008.

[50] Grimes, C. A., Kouzoudis, D., Ong, K. G., Crump, R., "Thin-film Magnetoelastic Microsensors for Remote Query Biomedical Monitoring", Biomedical Microdevices 2:1, p51-60, 1999.

[51] Borrmann, T., Private communication, 2008.

[52] Holt, R. C., "The Application of Acoustic Waves to Investigate the Viscoelastic Properties of Thin Films", MSc Thesis, Victoria University of Wellington, 2004.

[53] Zhen, J. Z., "Modulation Techniques of Chemical Sensors for Analyte Identification", MSc Thesis, Victoria University of Wellington, 2004.

[54] National Instruments Inc., "National Instruments - LabVIEW" $<$ http://www.ni.com/labview>, accessed 23/8/2008.

[55] Georgia State University, "HyperPhysics: Relative Humidity" $<$ http://hyperphysics.phy-astr.gsu.edu/hbase/kinetic/relhum.html>, accessed 20/8/2008. 
[56] Honeywell Sensing and Control, "HIH-4000-004 Product Page", $<\mathrm{http} / /$ sensing.honeywell.com/index.cfm?ci_id $=140301 \&$ la_id $=1 \& \mathrm{pr}$ id $=836$ $38>$, accessed 20/8/2008.

[57] Mahaveer, J. K., Grimes C. A., "A Wireless Magnetoelastic Micro-Sensor Array for Simultaneous Measurement of Temperature and Pressure", IEEE Transactions on Magnetics, Vol. 37, No. 4, p2022-2024, July 2001.

[58] Grimes, C. A., Mahaveer, K. J., Singh, R. S., Cai, Q., Mason, A., "Magnetoelastic Microsensors for Environmental Monitoring", Digest, IEEE International MEMS-01 Conference, Interlaken, Switzerland, January 2001.

[59] Albert, K. J., Lewis, N. S., Schauer, C. L., "Cross Selective Chemical Sensor Arrays", Chemical Reviews, Vol. 100, p2595-2626, 2000.

[60] South Bay Technology Inc., "South Bay Technology Inc. Website", $<$ http://www.southbaytech.com/consum2.shtml >, accessed 23/8/2008. 
